Supporting Information

\title{
Total Synthesis of the Echinodermatous Ganglioside LLG-3 Possessing the Biological Function of Promoting the Neurite Outgrowth
}

Yu-Fa Wu, ${ }^{\dagger}$ Yow-Fu Tsai, ${ }^{\dagger}{ }^{\dagger}$ Yuahn-Sieh Huang, ${ }^{*}$ and Jing-Feng Shih ${ }^{\dagger}$

${ }^{\dagger}$ Department of Chemistry, Chung Yuan Christian University, Chung Li District, Taoyuan 32023, Taiwan

* Department of Biology and Anatomy, National Defense Medical Center, Taipei, Taiwan

${ }^{\dagger}$ tsaiyofu@cycu.edu.tw; ${ }^{*}$ anatoman2001@yahoo.com.tw 


\section{Table of Contents}

I. Experimental Procedures 3

(A) General Materials and Methods: 3

(B) Synthesis of $\mathrm{N}$-acetylneuraminic acid derivative 2: 3

(C) Synthesis of N-acetylneuraminic acid derivative 20:

(D) Synthesis of glucose derivative 17: 8

(E) Synthesis of galactose derivative 21: 10

(F) Synthesis of the protected phytoceramide 16: 13

(G) Synthesis of the amino-disaccharide 3: 17

(H) Synthesis of the 4-OHGlc-PhytoCer 4:

(I) Completion of the total synthesis of the ganglioside LLG-3 (1): 21

II. Materials and Methods for Biological Studies: 24

(A) Results:

(B) Methods:

III. References

IV. Spectra of Compounds: $\quad 27$ 


\section{Experimental Procedures}

\section{(A) General Materials and Methods:}

Chemicals used in reaction were reagent grade and were used without further purification except where noted. All solvents used in reaction were obtained from E. Merck or Acros and were dried by stander procedures ${ }^{1}$ before use. Solvents for spectrometers were spectroscopy grade and were purchased from E. Merck Company. Solvents used for extraction, and chromatography were technical grade and were distilled prior to use. Reactions were monitored by thin-layer chromatography performed on $0.25 \mathrm{~mm}$ TLC aluminium plates of Silica Gel $60 \mathrm{~F}_{524}(\mathrm{E}$. Merck) and compound spots were visualized by UV light $(254 \mathrm{~nm})$ and by staining with a solution of $\mathrm{Ce}\left(\mathrm{NH}_{4}\right)_{2}\left(\mathrm{NO}_{3}\right)_{6}(0.5 \mathrm{~g})$ and $\left(\mathrm{NH}_{4}\right)_{6} \mathrm{Mo}_{7} \mathrm{O}_{4} \mathrm{H}_{2} \mathrm{O}(24.0 \mathrm{~g})$ in $6 \% \mathrm{H}_{2} \mathrm{SO}_{4}(500 \mathrm{~mL})$. Flash chromatography was carried out using E. Merck Silica Gel 60 (230-400 mesh, 111567.9025).

Melting points are uncorrected and were observed using a Yanagimoto Micromelting Point Apparatus. Optical rotations were performed using a Jasco P-1010 polarimeter at the indicated temperature. Infrared spectra were obtained with a JASCO FTIR-4200 and recorded as neat on KBr plates in $\mathrm{cm}^{-1} .{ }^{1} \mathrm{H}$ and ${ }^{13} \mathrm{C}$ NMR spectra were recorded with a Bruker Avance $300\left(300 \mathrm{MHz}\right.$ for ${ }^{1} \mathrm{H} ; 75 \mathrm{MHz}$ for $\left.{ }^{13} \mathrm{C}\right)$ and Bruker Avance II-400 (400 MHz for ${ }^{1} \mathrm{H}$; $100 \mathrm{MHz}$ for ${ }^{13} \mathrm{C}$ ) FT-NMR instrument. Chemical shifts $(\delta)$ are reported in ppm relative to tetramethylsilane ( $\left.\delta 0.00\right)$ or the residual proton of $\mathrm{CDCl}_{3}\left(\delta_{\mathrm{H}} 7.26, \delta_{\mathrm{C}} 77.0\right)$ as internal standard. COSY, HMQC, and HMBC spectra were applied to the detailed NMR assignments. Stereochemistry of compounds was determined using NOESY. High resolution mass spectra (HRMS) were recorded on a Bruker Daltonics Esquire 2000 mass spectrometer using ESI-TOF (electrospray ionization-time of flight).

(B) Synthesis of $\mathrm{N}$-acetylneuraminic acid derivative 2:
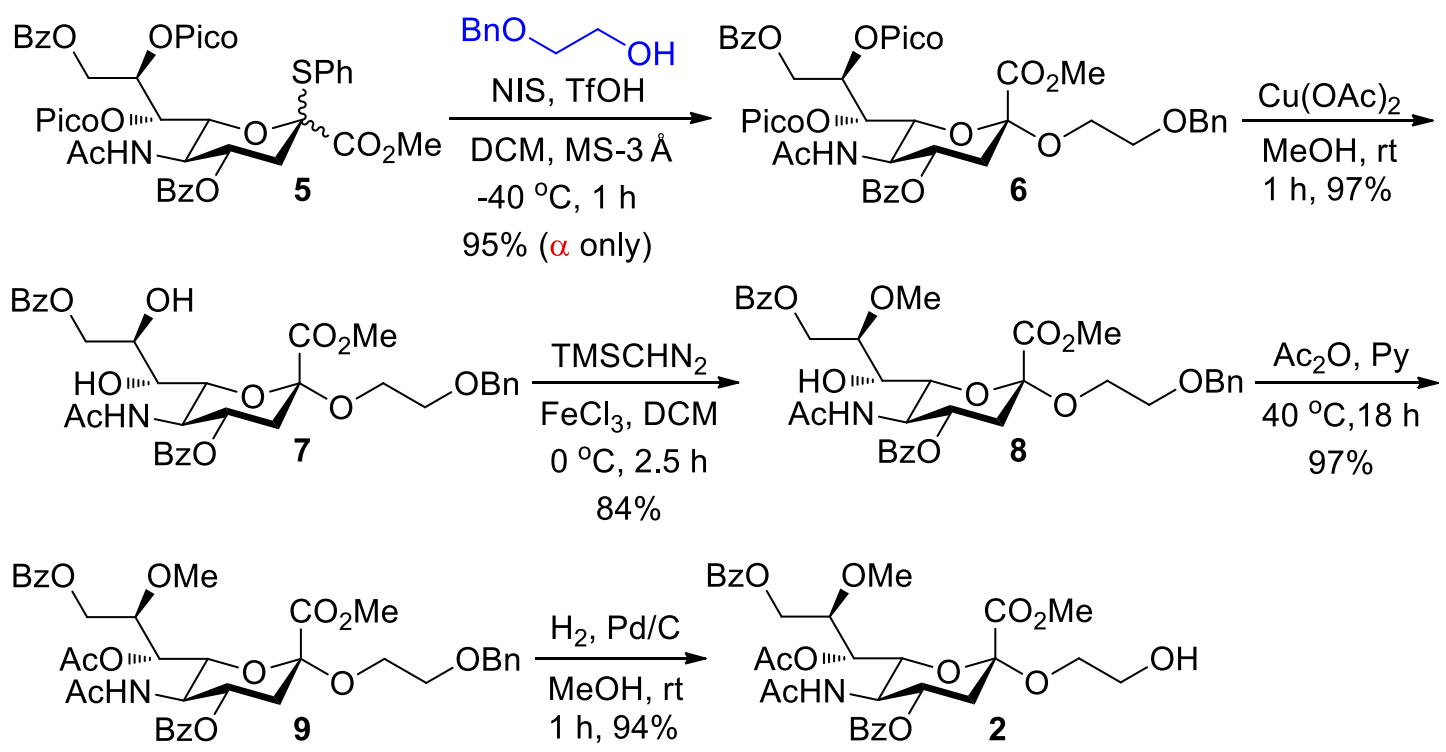

Scheme S1. Synthesis of $N$-acetylneuraminic acid derivative 2 .

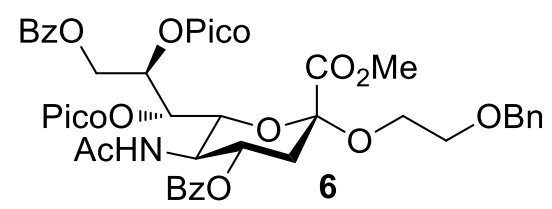

Methyl (2-(phenylmethoxy)ethyl 5-acetamido-4,9-di- $O$-benzoyl-3,5-dideoxy-7,8-di- $O$-picoloyl-D-glycero- $\alpha$-D-galacto-2-nonulopyranosid) onate (6) A mixture of the compound 5 ( $0.151 \mathrm{~g}, 0.18 \mathrm{mmol}), 2$-(benzyloxy)ethanol $(0.033 \mathrm{~g}, 0.22 \mathrm{mmol})$, and activated $3 \AA$ powdered molecular sieves $(0.331 \mathrm{~g})$ in anhydrous $\mathrm{CH}_{2} \mathrm{Cl}_{2}(3.6 \mathrm{~mL})$ was stirred at room temperature for $1 \mathrm{~h}$ under nitrogen to remove any trace amounts of water. The reaction mixture was then cooled to $-40{ }^{\circ} \mathrm{C}$ followed by addition of $N$-iodosuccinimide $(0.081 \mathrm{~g}, 0.36 \mathrm{mmol})$ and $\mathrm{TfOH}(0.023 \mathrm{~mL}, 0.27 \mathrm{mmol})$. Continuously stirred at this temperature for $1 \mathrm{~h}$, the reaction mixture was carefully quenched with triethylamine and then filtered through a short pad of Celite. The filtrate was washed with cold saturated aqueous $\mathrm{Na}_{2} \mathrm{~S}_{2} \mathrm{O}_{3}$, brine, dried over $\mathrm{MgSO}_{4}$, filtered, and concentrated in vacuo. The observed yellowish syrup was purified by flash column chromatography on silica gel using ethyl acetate as the eluent to give $0.151 \mathrm{~g}$ of a white solid 6 in $95 \%$ yield: $R_{f}=0.20$ (ethyl acetate); $\mathrm{mp}=70-71{ }^{\circ} \mathrm{C}$; $[\alpha]^{24} \mathrm{D}+59.4\left(\mathrm{c} 0.51, \mathrm{CHCl}_{3}\right.$ ); FT-IR (neat) $v_{\max } 3335,3064,2957,2873,1729,1681$, $1579,1547,1445,1372,1279,1119,753,710 \mathrm{~cm}^{-1} ;{ }^{1} \mathrm{H} \mathrm{NMR}\left(400 \mathrm{MHz}, \mathrm{CDCl}_{3}\right) \delta 8.76(\mathrm{~d}, J=4.2 \mathrm{~Hz}, 2 \mathrm{H}, \mathrm{ArH}), 8.15(\mathrm{dd}, J=7.6,3.4 \mathrm{~Hz}, 2 \mathrm{H}$, 
ArH), $7.94(\mathrm{dd}, J=9.6,7.6 \mathrm{~Hz}, 4 \mathrm{H}, \mathrm{ArH}), 7.87-7.79(\mathrm{~m}, 2 \mathrm{H}, \mathrm{ArH}), 7.53-7.45(\mathrm{~m}, 4 \mathrm{H}, \mathrm{ArH}), 7.39-7.29(\mathrm{~m}, 9 \mathrm{H}, \mathrm{ArH}), 6.16-6.12(\mathrm{~m}, 1 \mathrm{H}, \mathrm{H}-8), 6.07$ (dd, $J=8.4,1.6 \mathrm{~Hz}, 1 \mathrm{H}, \mathrm{H}-7), 5.69$ (d, $J=9.3 \mathrm{~Hz}, 1 \mathrm{H}, \mathrm{NH}), 5.19-5.12(\mathrm{~m}, 1 \mathrm{H}, \mathrm{H}-4), 4.95$ (dd, $J=12.3,2.6 \mathrm{~Hz}, 1 \mathrm{H}, \mathrm{H}-9 \mathrm{a}), 4.61,4.57$ (ABq, $J=$

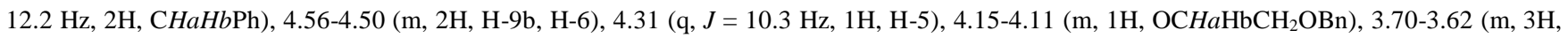
$\mathrm{OCHaHbCH} \mathrm{CBBn}_{2}, 3.49$ (s, 3H, $\mathrm{CO}_{2} \mathrm{CH}_{3}$ ), 2.83 (dd, $\left.J=12.6,4.7 \mathrm{~Hz}, 1 \mathrm{H}, \mathrm{H}-3 \mathrm{eq}\right), 2.12$ (t, $\left.J=12.6 \mathrm{~Hz}, 1 \mathrm{H}, \mathrm{H}-3 \mathrm{ax}\right), 1.81$ (s, $3 \mathrm{H}, \mathrm{Ac}$ ); ${ }^{13} \mathrm{C} \mathrm{NMR}$ $\left(100 \mathrm{MHz}, \mathrm{CDCl}_{3}\right) \delta 170.3(\mathrm{C}), 167.9(\mathrm{C}), 165.9(\mathrm{C}), 165.7$ (C), $163.8(\mathrm{C}), 163.7(\mathrm{C}), 149.6(\mathrm{CH}), 149.5(\mathrm{CH}), 147.3(\mathrm{C}), 147.1(\mathrm{C}), 138.0(\mathrm{C})$, $136.9(\mathrm{CH}), 136.8(\mathrm{CH}), 133.0(\mathrm{CH}), 132.7(\mathrm{CH}), 129.4(\mathrm{CH}), 129.3(\mathrm{CH}), 129.2(\mathrm{C}), 128.2(\mathrm{CH}), 128.0(\mathrm{CH}), 127.4(\mathrm{CH}), 127.3(\mathrm{CH}), 127.2$ $(\mathrm{CH}), 126.9(\mathrm{CH}), 126.8(\mathrm{CH}), 125.3(\mathrm{CH}), 98.9(\mathrm{C}), 72.8(\mathrm{CH}), 72.6\left(\mathrm{CH}_{2}\right), 71.6(\mathrm{CH}), 70.1(\mathrm{CH}), 69.6(\mathrm{CH}), 68.7\left(\mathrm{CH}_{2}\right), 64.2\left(\mathrm{CH}_{2}\right), 63.0\left(\mathrm{CH}_{2}\right)$, $52.2\left(\mathrm{CH}_{3}\right), 49.0(\mathrm{CH}), 37.9\left(\mathrm{CH}_{2}\right), 22.7\left(\mathrm{CH}_{3}\right)$; HRMS-ESI $[\mathrm{M}+\mathrm{Na}]^{+}$Calcd for $\mathrm{C}_{47} \mathrm{H}_{45} \mathrm{~N}_{3} \mathrm{O}_{14} \mathrm{Na} 898.2794$, Found 898.2800.

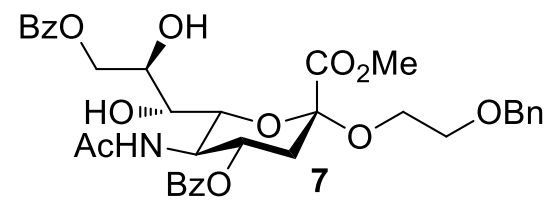

Methyl (2-(phenylmethoxy)ethyl 5-acetamido-4,9-di-O-benzoyl-3,5-dideoxy-D-glycero- $\boldsymbol{\alpha}$-D-galacto-2-nonulopyranosid)onate (7) To a stirring solution of compound $6(1.635 \mathrm{~g}, 1.87 \mathrm{mmol})$ in $\mathrm{MeOH}(9.3 \mathrm{~mL})$ was added $\mathrm{Cu}(\mathrm{OAc})_{2}(0.68 \mathrm{~mL}, 3.73 \mathrm{mmol})$ at $0{ }^{\circ} \mathrm{C}$. The mixture was warmed to room temperature and continuously stirred at this temperature for $1 \mathrm{~h}$. The reaction mixture was extracted with ethyl acetate, washed with cold $1 \mathrm{~N} \mathrm{HCl}$, brine, dried over $\mathrm{MgSO}_{4}$, and concentrated in vacuo to get $1.243 \mathrm{~g}$ of 7 as a white solid compound in $97 \%$ yield: $R_{f}=0.63$ (ethyl acetate); $\mathrm{mp}=58-59^{\circ} \mathrm{C} ;[\alpha]^{24} \mathrm{D}-34.7\left(\mathrm{c} 0.40, \mathrm{CHCl}_{3}\right.$ ); FT-IR (neat) $v_{\max } 3352,3072,2953,2870,1719,1663,1549,1447,1372,1276,1204$, 1117, 1037, $711 \mathrm{~cm}^{-1} ;{ }^{1} \mathrm{H}$ NMR (400 MHz, $\left.\mathrm{CDCl}_{3}\right) \delta 8.07(\mathrm{dd}, J=8.1,0.7 \mathrm{~Hz}, 2 \mathrm{H}, \mathrm{ArH}), 8.02(\mathrm{dd}, J=8.1,0.7 \mathrm{~Hz}, 2 \mathrm{H}, \mathrm{ArH}), 7.63(\mathrm{t}, J=7.4 \mathrm{~Hz}$, $1 \mathrm{H}, \mathrm{ArH}), 7.54(\mathrm{t}, J=7.4 \mathrm{~Hz}, 1 \mathrm{H}, \mathrm{ArH}), 7.48(\mathrm{t}, J=7.7 \mathrm{~Hz}, 2 \mathrm{H}, \mathrm{ArH}), 7.41(\mathrm{t}, J=7.7 \mathrm{~Hz}, 2 \mathrm{H}, \mathrm{ArH}), 7.34-7.33(\mathrm{~m}, 4 \mathrm{H}, \mathrm{ArH}), 7.30-7.27(\mathrm{~m}, 1 \mathrm{H}$, $\operatorname{ArH}), 6.17(\mathrm{~d}, J=7.7 \mathrm{~Hz}, 1 \mathrm{H}, \mathrm{NH}), 5.20$ (ddd, $J=12.8,10.2,4.8 \mathrm{~Hz}, 1 \mathrm{H}, \mathrm{H}-4), 4.82$ (d, $J=4.3 \mathrm{~Hz}, 1 \mathrm{H}, \mathrm{OH}-7), 4.71(\mathrm{~d}, J=12.0 \mathrm{~Hz}, 1 \mathrm{H}, \mathrm{H}-9 \mathrm{a})$, $4.57,4.53(\mathrm{ABq}, J=12.0 \mathrm{~Hz}, 2 \mathrm{H}, \mathrm{CH} a H b \mathrm{Ph}), 4.50$ (dd, $J=12.0,6.5 \mathrm{~Hz}, 1 \mathrm{H}, \mathrm{H}-9 \mathrm{~b}), 4.29-4.25$ (m, 1H, H-8), 4.14 (td, $J=10.2,7.7 \mathrm{~Hz}, 1 \mathrm{H}, \mathrm{H}-5)$, 4.03-3.98 (m, $1 \mathrm{H}, \mathrm{OCHaHbCH} 2 \mathrm{OBn}), 3.80$ (s, 3H, $\mathrm{CO}_{2} \mathrm{CH}_{3}$ ), 3.71-3.59 (m, 6H, OH-8, H-7, H-6, OCHaHbCH${ }_{2} \mathrm{OBn}$ ), 2.89 (dd, J = 12.8, $4.8 \mathrm{~Hz}$, $1 \mathrm{H}, \mathrm{H}-3 \mathrm{eq}), 2.25$ (t, $J=12.8 \mathrm{~Hz}, 1 \mathrm{H}, \mathrm{H}-3 \mathrm{ax}), 1.92$ (s, 3H, Ac); ${ }^{13} \mathrm{C}$ NMR (100 MHz, CDCl $) \delta 172.9$ (C), 169.2 (C), 166.6 (C), 166.4 (C), 137.7 (C), $133.4(\mathrm{CH}), 132.7(\mathrm{CH}), 129.9(\mathrm{C}), 129.5(\mathrm{CH}), 129.4(\mathrm{CH}), 128.9(\mathrm{C}), 128.4(\mathrm{CH}), 128.1(\mathrm{CH}), 127.5(\mathrm{CH}), 127.4(\mathrm{CH}), 98.4(\mathrm{C}), 73.3(\mathrm{CH})$, $72.8\left(\mathrm{CH}_{2}\right), 69.7(\mathrm{CH}), 69.4(\mathrm{CH}), 68.9(\mathrm{CH}), 68.5\left(\mathrm{CH}_{2}\right), 66.7\left(\mathrm{CH}_{2}\right), 63.5\left(\mathrm{CH}_{2}\right), 53.0\left(\mathrm{CH}_{3}\right), 50.0(\mathrm{CH}), 37.0\left(\mathrm{CH}_{2}\right), 22.6\left(\mathrm{CH}_{3}\right) ; \mathrm{HRMS}-\mathrm{ESI}[\mathrm{M}$ $+\mathrm{Na}]^{+}$Calcd for $\mathrm{C}_{35} \mathrm{H}_{39} \mathrm{NO}_{12} \mathrm{Na} 688.2364$, Found 688.2363.

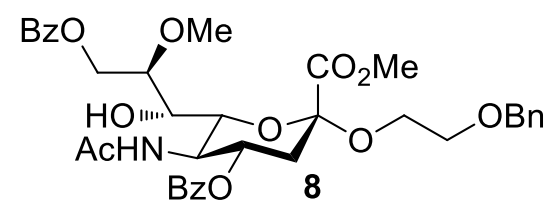

Methyl (2-(phenylmethoxy)ethyl 5-acetamido-4,9-di-O-benzoyl-3,5-dideoxy-8-O-methyl-D-glycero- $\alpha$-D-galacto-2-nonulopyranosid)onate (8) To a stirring solution of compound $7(0.420 \mathrm{~g}, 0.63 \mathrm{mmol})$ in $\mathrm{CH}_{2} \mathrm{Cl}_{2}(9.0 \mathrm{~mL})$ was added $\mathrm{FeCl}_{3}(0.180 \mathrm{~mL}, 1.14 \mathrm{mmol})$ and TMSCHN $2(0.63$ $\mathrm{mL}, 1.26 \mathrm{mmol}$ ) at $0{ }^{\circ} \mathrm{C}$. Continuously stirred at this temperature for $2.5 \mathrm{~h}$, the reaction mixture was carefully quenched with $\mathrm{MeOH}$ and then filtered through a short pad of Celite. The filtrate was washed with cold saturated aqueous $\mathrm{NaHCO}_{3}$, brine, dried over $\mathrm{MgSO}_{4}$, filtered, and concentrated in vасио. The resulting yellowish syrup residue was purified by flash column chromatography on silica gel using ethyl acetate and hexanes $(1: 1, \mathrm{v} / \mathrm{v})$ as the eluent to get $0.360 \mathrm{~g}$ of 8 as a white solid compound in $84 \%$ yield: $R_{f}=0.30($ ethyl acetate: hexanes $=1: 1(\mathrm{v} / \mathrm{v})$ ); $\mathrm{mp}=60-$ $61{ }^{\circ} \mathrm{C} ;[\alpha]^{24}{ }_{\mathrm{D}}-20.3$ (c 0.27, $\mathrm{CHCl}_{3}$ ); FT-IR (neat) $v_{\max } 3355,3068,2944,1719,1658,1547,1447,1372,1276,1110,1032,710 \mathrm{~cm}^{-1} ;{ }^{1} \mathrm{H} \mathrm{NMR}(400$ $\left.\mathrm{MHz}, \mathrm{CDCl}_{3}\right) \delta 8.04-8.00(\mathrm{~m}, 4 \mathrm{H}, \mathrm{ArH}), 7.61(\mathrm{t}, J=7.4 \mathrm{~Hz}, 1 \mathrm{H}, \mathrm{ArH}), 7.53(\mathrm{t}, J=7.4 \mathrm{~Hz}, 1 \mathrm{H}, \mathrm{ArH}), 7.48-7.39(\mathrm{~m}, 4 \mathrm{H}, \mathrm{ArH}), 7.34-7.27(\mathrm{~m}, 5 \mathrm{H}$, ArH), $6.08(\mathrm{~d}, J=8.2 \mathrm{~Hz}, 1 \mathrm{H}, \mathrm{NH}), 5.33-5.27(\mathrm{~m}, 1 \mathrm{H}, \mathrm{H}-4), 4.98(\mathrm{dd}, J=11.9,1.8 \mathrm{~Hz}, 1 \mathrm{H}, \mathrm{H}-9 \mathrm{a}), 4.62(\mathrm{~d}, J=5.4 \mathrm{~Hz}, 1 \mathrm{H}, \mathrm{OH}-7), 4.58,4.55$ $(\mathrm{ABq}, J=12.4 \mathrm{~Hz}, 2 \mathrm{H}, \mathrm{CHaHbPh}), 4.39(\mathrm{dd}, J=11.9,4.0 \mathrm{~Hz}, 1 \mathrm{H}, \mathrm{H}-9 \mathrm{~b}), 4.17(\mathrm{td}, J=10.5,8.2 \mathrm{~Hz}, 1 \mathrm{H}, \mathrm{H}-5), 4.07(\mathrm{dt}, J=10.2,5.0 \mathrm{~Hz}, 1 \mathrm{H}$, $\mathrm{OCHaHbCH} \mathrm{OBn}_{2}, 3.80$ (s, 3H, $\mathrm{CO}_{2} \mathrm{CH}_{3}$ ), 3.78-3.74 (m, 3H, H-8, H-6, OCHaHbCH $\mathrm{CH}_{2} \mathrm{OBn}$ ), 3.66-3.63 (m, 3H, H-7, OCHaHbCH $\mathrm{OBn}_{2}, 3.54$ (s, $\left.3 \mathrm{H}, \mathrm{OCH}_{3}\right), 2.83(\mathrm{dd}, J=12.6,4.8 \mathrm{~Hz}, 1 \mathrm{H}, \mathrm{H}-3 \mathrm{eq}), 2.17$ (t, $\left.J=12.6 \mathrm{~Hz}, 1 \mathrm{H}, \mathrm{H}-3 \mathrm{ax}\right), 1.90(\mathrm{~s}, 3 \mathrm{H}, \mathrm{Ac}){ }^{13} \mathrm{C} \mathrm{NMR}\left(100 \mathrm{MHz}, \mathrm{CDCl}_{3}\right) \delta 172.7(\mathrm{C})$, $168.4(\mathrm{C}), 166.7(\mathrm{C}), 166.2(\mathrm{C}), 138.0(\mathrm{C}), 133.4(\mathrm{CH}), 132.7(\mathrm{CH}), 130.2(\mathrm{C}), 129.5(\mathrm{CH}), 129.4(\mathrm{CH}), 128.9(\mathrm{C}), 128.3(\mathrm{CH}), 128.2(\mathrm{CH}), 127.6$ $(\mathrm{CH}), 127.4(\mathrm{CH}), 98.6(\mathrm{C}), 77.3(\mathrm{CH}), 73.3(\mathrm{CH}), 73.0\left(\mathrm{CH}_{2}\right), 69.6(\mathrm{CH}), 68.8\left(\mathrm{CH}_{2}\right), 67.8(\mathrm{CH}), 63.5\left(\mathrm{CH}_{2}\right), 63.2\left(\mathrm{CH}_{2}\right), 58.1\left(\mathrm{CH}_{3}\right), 52.5\left(\mathrm{CH}_{3}\right)$, $50.8(\mathrm{CH}), 37.4\left(\mathrm{CH}_{2}\right), 22.7\left(\mathrm{CH}_{3}\right)$; HRMS-ESI $[\mathrm{M}+\mathrm{Na}]^{+} \mathrm{Calcd}$ for $\mathrm{C}_{36} \mathrm{H}_{41} \mathrm{NO}_{12} \mathrm{Na} 702.2521$, Found 702.2525. 


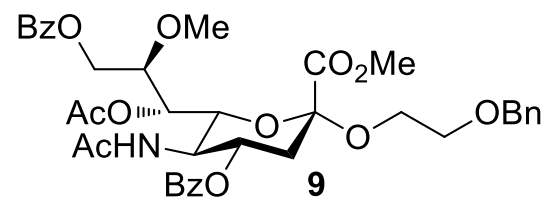

Methyl (2-(phenylmethoxy)ethyl $\quad 5$-acetamido-7-O-acetyl-4,9-di-O-benzoyl-3,5-dideoxy-8-O-methyl-D-glycero- $\quad \alpha \quad$-D-galacto-2nonulopyranosid)onate (9) To a stirring solution of compound $8(0.937 \mathrm{~g}, 1.38 \mathrm{mmol})$ in pyridine $(7.0 \mathrm{~mL})$ was added $\mathrm{Ac}_{2} \mathrm{O}(0.19 \mathrm{~mL}, 2.07$ $\mathrm{mmol})$ at $0{ }^{\circ} \mathrm{C}$. The mixture was warmed to room temperature and continuously stirred at this temperature for $18 \mathrm{~h}$. The reaction mixture was extracted with ethyl acetate, washed with cold $1 \mathrm{~N} \mathrm{HCl}$, cold saturated aqueous $\mathrm{NaHCO}_{3}$, brine, dried over $\mathrm{MgSO}_{4}$, and concentrated in vacuo to obtain $0.965 \mathrm{~g}$ of 9 as a white solid compound in $97 \%$ yield: $R_{f}=0.20$ (ethyl acetate: hexanes $=1: 1(\mathrm{v} / \mathrm{v})$ ); $\mathrm{mp}=59-60{ }^{\circ} \mathrm{C} ;[\alpha]^{24} \mathrm{D}+34.2(\mathrm{c} 0.46$, $\mathrm{CHCl}_{3}$ ); FT-IR (neat) $v_{\max } 3369,3065,2948,1721,1539,1447,1370,1276,1206,1110,1034,712 \mathrm{~cm}^{-1} ;{ }^{1} \mathrm{H} \mathrm{NMR}\left(400 \mathrm{MHz}, \mathrm{CDCl}_{3}\right) \delta 8.07(\mathrm{~d}, J$ $=7.2 \mathrm{~Hz}, 2 \mathrm{H}, \mathrm{ArH}), 7.99$ (d, J = 7.6 Hz, 2H, ArH), 7.58-7.53 (m, 2H, ArH), 7.44-7.32 (m, 9H, ArH), 5.27 (dd, $J=9.0,1.4 \mathrm{~Hz}, 1 \mathrm{H}, \mathrm{H}-7), 5.18(\mathrm{~d}, J$ $=9.9 \mathrm{~Hz}, 1 \mathrm{H}, \mathrm{NH}), 5.18-5.13(\mathrm{~m}, 1 \mathrm{H}, \mathrm{H}-4), 4.72(\mathrm{dd}, J=12.2,3.4 \mathrm{~Hz}, 1 \mathrm{H}, \mathrm{H}-9 \mathrm{a}), 4.57\left(\mathrm{~s}, 2 \mathrm{H}, \mathrm{CH}_{2} \mathrm{Ph}\right), 4.32-4.24(\mathrm{~m}, 1 \mathrm{H}, \mathrm{H}-5), 4.22(\mathrm{dd}, J=10.8$, $1.4 \mathrm{~Hz}, 1 \mathrm{H}, \mathrm{H}-6), 4.16$ (dd, $J=12.2,4.6 \mathrm{~Hz}, 1 \mathrm{H}, \mathrm{H}-9 \mathrm{~b}), 4.04-3.99\left(\mathrm{~m}, 1 \mathrm{H}, \mathrm{OCHaHbCH} \mathrm{HBBn}_{2}, 3.92-3.88(\mathrm{~m}, 1 \mathrm{H}, \mathrm{H}-8), 3.83\left(\mathrm{~s}, 3 \mathrm{H}, \mathrm{CO}_{2} \mathrm{CH}_{3}\right)\right.$, 3.73-3.63 (m, 3H, OCHaHbCH $\mathrm{OBn}_{2}, 3.58$ (s, 3H, $\mathrm{OCH}_{3}$ ), 2.87 (dd, $\left.J=12.5,4.7 \mathrm{~Hz}, 1 \mathrm{H}, \mathrm{H}-3 \mathrm{eq}\right), 2.14$ (s, 3H, Ac), 2.12 (t, $J=12.5 \mathrm{~Hz}, 1 \mathrm{H}, \mathrm{H}-$ 3ax), 1.78 (s, 3H, Ac); ${ }^{13} \mathrm{C}$ NMR (100 MHz, $\left.\mathrm{CDCl}_{3}\right) \delta 170.4$ (C), $170.0(\mathrm{C}), 168.3(\mathrm{C}), 166.3(\mathrm{C}), 166.2(\mathrm{C}), 138.0(\mathrm{C}), 133.2(\mathrm{CH}), 132.9(\mathrm{CH})$, $129.8(\mathrm{C}), 129.6(\mathrm{CH}), 129.5(\mathrm{CH}), 129.2(\mathrm{C}), 128.3(\mathrm{CH}), 128.2(\mathrm{CH}), 128.2(\mathrm{CH}), 127.5(\mathrm{CH}), 127.5(\mathrm{CH}), 98.8(\mathrm{C}), 76.3(\mathrm{CH}), 73.0(\mathrm{CH})$, $72.3(\mathrm{CH}), 70.5(\mathrm{CH}), 68.7\left(\mathrm{CH}_{2}\right), 68.5(\mathrm{CH}), 63.8\left(\mathrm{CH}_{2}\right), 62.7\left(\mathrm{CH}_{2}\right), 58.4\left(\mathrm{CH}_{3}\right), 52.5\left(\mathrm{CH}_{3}\right), 48.8(\mathrm{CH}), 37.6\left(\mathrm{CH}_{2}\right), 22.9\left(\mathrm{CH}_{3}\right), 20.8\left(\mathrm{CH}_{3}\right)$; HRMS-ESI [M + Na] $]^{+}$Calcd for $\mathrm{C}_{38} \mathrm{H}_{43} \mathrm{NO}_{13} \mathrm{Na} 744.2627$, Found 744.2626

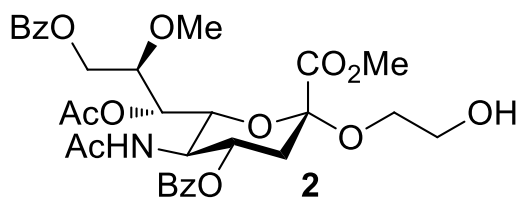

Methyl (2-hydroxyethyl 5-acetamido-7-O-acetyl-4,9-di-O-benzoyl-3,5-dideoxy-8-O-methyl-D-glycero- $\alpha$-D-galacto-2-nonulopyranosid)onate (2) To a solution of $9(0.950 \mathrm{~g}, 1.32 \mathrm{mmol})$ in $\mathrm{MeOH}(13 \mathrm{~mL})$ was added $10 \% \mathrm{Pd} / \mathrm{C}(0.052 \mathrm{~g})$ at room temperature. The reaction mixture was stirred under hydrogen balloon at room temperature for $1 \mathrm{~h}$. The $\mathrm{Pd} / \mathrm{C}$ was removed through a short $\mathrm{pad}$ of $\mathrm{SiO}_{2} / \mathrm{Celite}$ and the filter was washed with ethyl acetate. The filtrate was concentrated in vacuo to obtain $0.782 \mathrm{~g}$ of 2 as a white solid compound in $94 \%$ yield: $R_{f}=0.10$ (ethyl acetate: hexanes $=1: 1(\mathrm{v} / \mathrm{v})) ; \mathrm{mp}=144-145{ }^{\circ} \mathrm{C} ;[\alpha]^{24}+39.3\left(\mathrm{c} 0.39, \mathrm{CHCl}_{3}\right)$; FT-IR (neat) $v_{\max } 3364,3068,2952,2839,1721,1670,1545,1447,1373$, $1278,1221,1119,1035,755,714 \mathrm{~cm}^{-1}$; ${ }^{1} \mathrm{H}$ NMR $\left(400 \mathrm{MHz}, \mathrm{CDCl}_{3}\right) \delta 8.08(\mathrm{~d}, J=7.2 \mathrm{~Hz}, 2 \mathrm{H}, \mathrm{ArH}), 7.99(\mathrm{~d}, J=7.2 \mathrm{~Hz}, 2 \mathrm{H}, \mathrm{ArH}), 7.58-7.54(\mathrm{~m}$, $2 \mathrm{H}, \mathrm{ArH}), 7.46-7.41(\mathrm{~m}, 4 \mathrm{H}, \mathrm{ArH}), 5.28(\mathrm{dd}, J=9.2,1.2 \mathrm{~Hz}, 1 \mathrm{H}, \mathrm{H}-7), 5.25(\mathrm{~d}, J=10.5 \mathrm{~Hz}, 1 \mathrm{H}, \mathrm{NH}), 5.18(\mathrm{ddd}, J=12.5,9.9,4.6 \mathrm{~Hz}, 1 \mathrm{H}, \mathrm{H}-4)$, 4.76 (dd, $J=12.3,3.2 \mathrm{~Hz}, 1 \mathrm{H}, \mathrm{H}-9 \mathrm{a}), 4.31$ (dd, $J=10.7,1.2 \mathrm{~Hz}, 1 \mathrm{H}, \mathrm{H}-6), 4.29-4.22$ (m, 1H, H-5), 4.17 (dd, $J=12.3,4.6$ Hz, 1H, H-9b), 3.983.90 (m, 2H, H-8, OCHaHbCH$\left.{ }_{2} \mathrm{OH}\right), 3.88$ (s, 3H, $\left.\mathrm{CO}_{2} \mathrm{CH}_{3}\right), 3.75-3.74\left(\mathrm{~m}, 2 \mathrm{H}, \mathrm{OCHaHbCH} \mathrm{OHH}_{2} \mathrm{O}, 3.70-3.65\left(\mathrm{~m}, 1 \mathrm{H}, \mathrm{OCHaHbCH}_{2} \mathrm{OBn}\right), 3.58(\mathrm{~s}\right.$, $\left.3 \mathrm{H}, \mathrm{OCH}_{3}\right), 2.85(\mathrm{dd}, J=12.5,4.6 \mathrm{~Hz}, 1 \mathrm{H}, \mathrm{H}-3 \mathrm{eq}), 2.33(\mathrm{t}, J=5.0 \mathrm{~Hz}, 1 \mathrm{H}, \mathrm{OH}), 2.16(\mathrm{~s}, 3 \mathrm{H}, \mathrm{Ac}), 2.12(\mathrm{t}, J=12.5 \mathrm{~Hz}, 1 \mathrm{H}, \mathrm{H}-3 \mathrm{ax}), 1.79(\mathrm{~s}, 3 \mathrm{H}$, Ac) $;^{13} \mathrm{C}$ NMR (100 MHz, $\left.\mathrm{CDCl}_{3}\right) \delta 170.6(\mathrm{C}), 170.1(\mathrm{C}), 168.5(\mathrm{C}), 166.4(\mathrm{C}), 166.4(\mathrm{C}), 133.4(\mathrm{CH}), 133.1(\mathrm{CH}), 129.8(\mathrm{C}), 129.7(\mathrm{CH}), 129.7$ $(\mathrm{CH}), 129.5(\mathrm{CH}), 129.2(\mathrm{C}), 128.5(\mathrm{CH}), 128.4(\mathrm{CH}), 128.4(\mathrm{CH}), 98.8(\mathrm{C}), 76.5(\mathrm{CH}), 72.5(\mathrm{CH}), 70.3(\mathrm{CH}), 68.4(\mathrm{CH}), 66.3\left(\mathrm{CH}_{2}\right), 62.7\left(\mathrm{CH}_{2}\right)$, $61.5\left(\mathrm{CH}_{2}\right), 58.6\left(\mathrm{CH}_{3}\right), 52.8\left(\mathrm{CH}_{3}\right), 49.0(\mathrm{CH}), 37.7\left(\mathrm{CH}_{2}\right), 22.9\left(\mathrm{CH}_{3}\right), 20.9\left(\mathrm{CH}_{3}\right)$; HRMS-ESI $[\mathrm{M}+\mathrm{Na}]^{+} \mathrm{Calcd}$ for $\mathrm{C}_{31} \mathrm{H}_{37} \mathrm{NO}_{13} \mathrm{Na}^{6} 64.2157$, Found 654.2158 . 


\section{(C) Synthesis of $N$-acetylneuraminic acid derivative 20:}
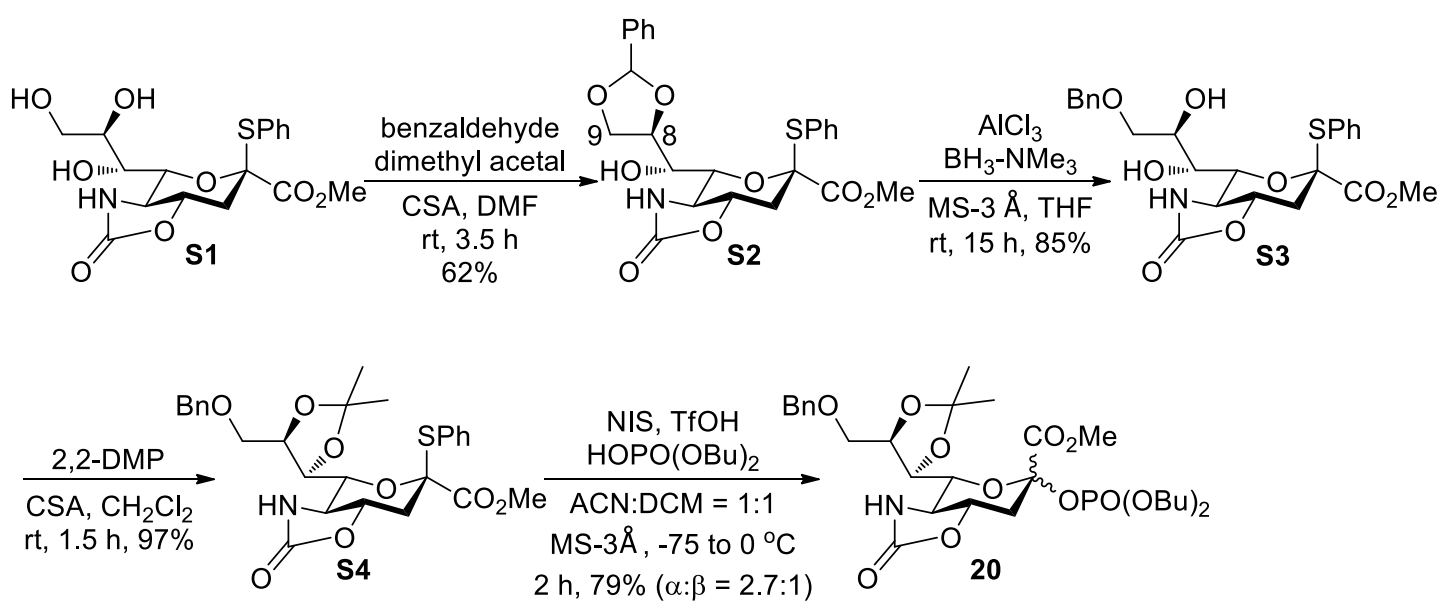

Scheme S2. Synthesis of $N$-acetylneuraminic acid derivative 20.

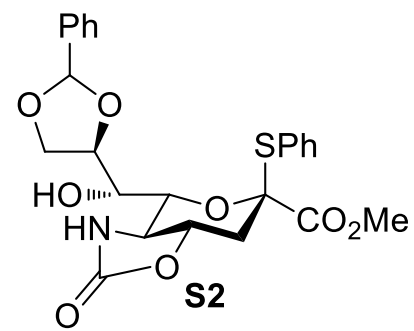

Methyl (phenyl 5-amino-8,9-O-benzylidene-5-N,4-O-carbonyl-3,5-dideoxy-2-thio- D-glycero-ß-D-galacto-2-nonulopyranosid)onate (S2) To a stirring solution of compound $\mathbf{S 1}(7.007 \mathrm{~g}, 17.56 \mathrm{mmol})$ in DMF $(58 \mathrm{~mL})$ was added benzaldehyde dimethyl acetal $(5.33 \mathrm{~mL}, 35.12 \mathrm{mmol})$ and CSA $(4.070 \mathrm{~g}, 17.56 \mathrm{mmol})$ at $0{ }^{\circ} \mathrm{C}$. The mixture was warmed to room temperature and continuously stirred at this temperature for $3.5 \mathrm{~h}$. The reaction mixture was carefully quenched with triethylamine and extracted with ethyl acetate, washed with cold $1 \mathrm{~N} \mathrm{HCl}$, brine, dried over $\mathrm{MgSO}_{4}$, and concentrated in vacuo. The resulting yellowish syrup residue was purified by flash column chromatography on silica gel using ethyl acetate and hexanes $(1: 1, \mathrm{v} / \mathrm{v})$ then ethyl acetate and hexanes $(4: 1, \mathrm{v} / \mathrm{v})$ as the eluent to get $5.313 \mathrm{~g}$ of $\mathbf{S} 2$ as a white solid compound in $62 \%$ yield: $R_{f}=$ 0.50 (MeOH: $\mathrm{CH}_{2} \mathrm{Cl}_{2}=1: 9$ (v/v)); FT-IR (neat) $v_{\max } 3330,3018,2948,2914,1757,1642,1550,1445,1393,1302,1242,1178,1095,1071,1012$, 939, 867, 755, $698 \mathrm{~cm}^{-1}$; ${ }^{1} \mathrm{H}$ NMR (400 MHz, $\left.\mathrm{CDCl}_{3}\right) \delta$ 7.49-7.29 (m, 20H, ArH), $5.93(\mathrm{~s}, 1 \mathrm{H}, \mathrm{CHPh}), 5.87(\mathrm{~s}, 1 \mathrm{H}, \mathrm{CHPh}), 5.76(\mathrm{~s}, 1 \mathrm{H}, \mathrm{NH}), 5.74$ (s, 1H, NH), 4.74-4.65 (m, 2H, H-4), 4.46 (dd, $J=10.3,6.3 \mathrm{~Hz}, 1 \mathrm{H}, \mathrm{H}-6$ ), 4.42 (dd, $J=10.2,5.6 \mathrm{~Hz}, 1 \mathrm{H}, \mathrm{H}-6$ ), 4.36 (dd, $J=8.8,4.1 \mathrm{~Hz}, 1 \mathrm{H}, \mathrm{H}-9$ ), $4.29(\mathrm{dd}, J=8.8,6.2 \mathrm{~Hz}, 1 \mathrm{H}, \mathrm{H}-9), 4.20-4.15$ (m, 2H, H-8), 4.12-4.03 (m, 2H, H-9), 3.74-3.62 (m, 2H, H-7), $3.66\left(\mathrm{~s}, 6 \mathrm{H}, \mathrm{CO}_{2} \mathrm{CH}_{3}\right), 3.45(\mathrm{t}, J=$ $10.3 \mathrm{~Hz}, 1 \mathrm{H}, \mathrm{H}-5), 3.34$ (t, $J=10.2 \mathrm{~Hz}, 1 \mathrm{H}, \mathrm{H}-5), 3.00-2.89(\mathrm{~m}, 4 \mathrm{H}), 2.36(\mathrm{t}, J=13.0 \mathrm{~Hz}, 1 \mathrm{H}), 2.34(\mathrm{t}, J=13.0 \mathrm{~Hz}, 1 \mathrm{H}) ; \mathrm{HRMS}-\mathrm{ESI}[\mathrm{M}+\mathrm{Na}]^{+}$ Calcd for $\mathrm{C}_{24} \mathrm{H}_{25} \mathrm{NO}_{8} \mathrm{SNa} 510.1193$, Found 510.1193 .

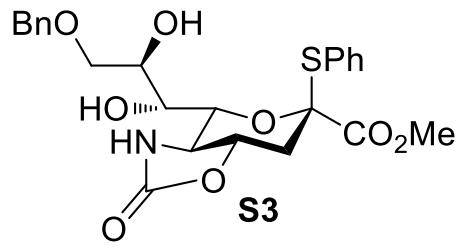

Methyl (phenyl 5-amino-9-O-benzyl-5-N,4-O-carbonyl-3,5-dideoxy-2-thio-D-glycero- $\beta$-D-galacto-2-nonulopyranosid)onate (S3) A mixture of the $\mathbf{S 2}(3.101 \mathrm{~g}, 6.36 \mathrm{mmol})$ and activated $3 \AA$ A powdered molecular sieves $(3.121 \mathrm{~g})$ in anhydrous THF (70 $\mathrm{mL})$ was stirred at room temperature for $1 \mathrm{~h}$ under nitrogen to remove any trace amounts of water. The reaction mixture was then cooled to $0{ }^{\circ} \mathrm{C}$ followed by addition of aluminum chloride $(5.069 \mathrm{~g}, 38.13 \mathrm{mmol})$ and borane trimethylamine complex $(2.782 \mathrm{~g}, 38.13 \mathrm{mmol})$. The mixture was warmed to room temperature and continuously stirred at this temperature for $15 \mathrm{~h}$. The reaction mixture was carefully quenched with $1 \mathrm{M} \mathrm{H}_{2} \mathrm{SO}_{4}$ at $0{ }^{\circ} \mathrm{C}$ for $0.5 \mathrm{~h}$ and extracted with ethyl acetate, washed with cold saturated aqueous $\mathrm{NaHCO}_{3}$, brine, dried over $\mathrm{MgSO}_{4}$, filtered, and concentrated in vacuo. The observed yellowish syrup was purified by flash column chromatography on silica gel using ethyl acetate and $\mathrm{CH}_{2} \mathrm{Cl}_{2}(1: 1$, v/v) as the eluent to give $2.640 \mathrm{~g}$ of a white solid S3 in 85\% yield: $R_{f}=0.25$ (ethyl acetate: hexanes $=2: 1(\mathrm{v} / \mathrm{v})$ ); $\mathrm{mp}=114-115{ }^{\circ} \mathrm{C}$; $[\alpha]^{24} \mathrm{D}-107.7\left(\mathrm{c} 0.36, \mathrm{CHCl}_{3}\right) ;$ FT-IR (neat) $v_{\max } 3385,3061$, $3021,2920,2865,1758,1444,1364,1303,1260,1178,1143,1104,1067,1013,939,868,752,695 \mathrm{~cm}^{-1} ;{ }^{1} \mathrm{H} \mathrm{NMR}(400 \mathrm{MHz}, \mathrm{CDCl}) \delta 7.48-7.32$ 
(m, $10 \mathrm{H}, \mathrm{ArH}), 6.04$ (s, 1H, NH), 4.68 (ddd, $J=12.4,11.5,3.8 \mathrm{~Hz}, 1 \mathrm{H}, \mathrm{H}-4), 4.57$ (s, 2H, CH $2 \mathrm{Ph}$ ), 4.45 (dd, $J=9.8,5.8 \mathrm{~Hz}, 1 \mathrm{H}, \mathrm{H}-6), 3.84-3.78$ (m, 1H, H-8), 3.76-3.61 (m, 3H, H-9, H-7), 3.63 (s, 3H), 3.42 (t, $J=10.3 \mathrm{~Hz}, 1 \mathrm{H}, \mathrm{H}-5$ ), 3.04 (bs, 1H, OH), 2.97 (d, $J=5.1 \mathrm{~Hz}, 1 \mathrm{H}, \mathrm{OH}), 2.91$ (dd,

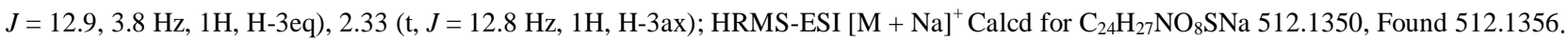

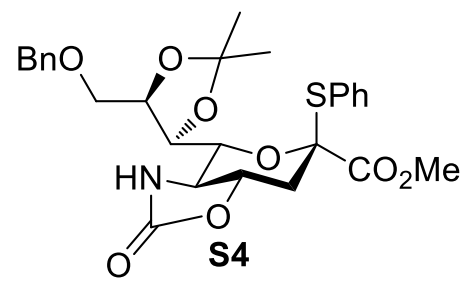

Methyl (phenyl 5-amino-9-O-benzyl-5- $N, 4-O$-carbonyl-3,5-dideoxy-7,8- $O$-isopropylidene-2-thio-D-glycero-p-D-galacto-2-nonulopyranosid) onate (S4) To a stirring solution of compound $\mathbf{S 3}(2.000 \mathrm{~g}, 4.09 \mathrm{mmol})$ in 2,2-dimethoxypropane $/ \mathrm{CH}_{2} \mathrm{Cl}_{2}(50 \mathrm{~mL}, 4 / 1$ (v/v)) was added CSA $(0.949 \mathrm{~g}, 4.09 \mathrm{mmol})$ at $0{ }^{\circ} \mathrm{C}$. The mixture was warmed to room temperature and continuously stirred at this temperature for $3 \mathrm{~h}$. The reaction mixture was carefully quenched with $\mathrm{Et}_{3} \mathrm{~N}(5.0 \mathrm{~mL})$ and concentrated in vacuo. The observed yellowish syrup was purified by flash column chromatography on silica gel using ethyl acetate and hexanes $(1: 1, \mathrm{v} / \mathrm{v})$ as the eluent to give $2.102 \mathrm{~g}$ of a white solid $\mathbf{S} 4$ in $97 \%$ yield: $R_{f}=0.45$ (ethyl acetate: hexanes $=2: 1(\mathrm{v} / \mathrm{v})) ; \mathrm{mp}=166-167^{\circ} \mathrm{C} ;[\alpha]_{\mathrm{D}}^{24}-80.2\left(\mathrm{c} 0.34, \mathrm{CHCl}_{3}\right)$; FT-IR (neat) $v_{\max } 3292,2987,2928,1766,1445,1374,1305$, 1232, 1165, 1085, 1011, 931, 870, $695 \mathrm{~cm}^{-1} ;{ }^{1} \mathrm{H}$ NMR (400 MHz, $\left.\mathrm{CDCl}_{3}\right) \delta$ 7.48-7.27 (m, 10H, ArH), $5.43(\mathrm{~s}, 1 \mathrm{H}, \mathrm{NH}), 4.71-4.65$ (m, 1H, H-4), $4.56,4.55(\mathrm{ABq}, J=12.3 \mathrm{~Hz}, 2 \mathrm{H}, \mathrm{CHaHbPh}), 4.50-4.45$ (m, 1H, H-8), 4.43 (dd, $J=10.2,2.2 \mathrm{~Hz}, 1 \mathrm{H}, \mathrm{H}-6), 4.21(\mathrm{dd}, J=6.8,2.2 \mathrm{~Hz}, 1 \mathrm{H}, \mathrm{H}-7)$, 3.83-3.75 (m, 2H, H-9), 3.67 (s, 3H, $\mathrm{CO}_{2} \mathrm{CH}_{3}$ ), 3.55 (t, $\left.J=10.2 \mathrm{~Hz}, 1 \mathrm{H}, \mathrm{H}-5\right), 2.67$ (dd, $\left.J=13.0,3.6 \mathrm{~Hz}, 1 \mathrm{H}, \mathrm{H}-3 \mathrm{eq}\right), 2.18$ (t, $J=13.0 \mathrm{~Hz}, 1 \mathrm{H}, \mathrm{H}-$ 3ax), 1.47 (s, 3H, $\left.\left(\mathrm{CH}_{3}\right)_{2} \mathrm{C}\right), 1.36\left(\mathrm{~s}, 3 \mathrm{H},\left(\mathrm{CH}_{3}\right)_{2} \mathrm{C}\right)$; HRMS-ESI $[\mathrm{M}+\mathrm{Na}]^{+} \mathrm{Calcd}$ for $\mathrm{C}_{27} \mathrm{H}_{31} \mathrm{NO}_{8} \mathrm{SNa} 552.1663$, Found 552.1668.

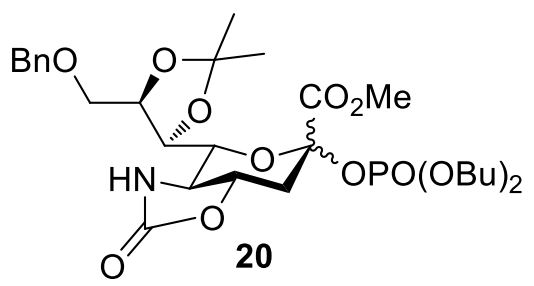

Methyl (5-amino-9-O-benzyl-5-N,4-O-carbonyl-3,5-dideoxy-2-(diphenylphosphoryl)-7,8-O-isopropylidene-D-glycero- $\beta$-D-galacto-2nonulopyranosid)onate (20) A mixture of the compound $\mathbf{S 4}(0.761 \mathrm{~g}, 1.44 \mathrm{mmol})$, dibutyl phosphate $(0.903 \mathrm{~g}$, $4.31 \mathrm{mmol})$, and activated $3 \AA$ powdered molecular sieves $(1.183 \mathrm{~g})$ in anhydrous $\mathrm{CH}_{2} \mathrm{Cl}_{2} / \mathrm{ACN}(14 \mathrm{~mL}, 1 / 1(\mathrm{v} / \mathrm{v}))$ was stirred at room temperature for $2 \mathrm{~h}$ under nitrogen to remove any trace amounts of water. The reaction mixture was then cooled to $-70{ }^{\circ} \mathrm{C}$ followed by addition of $\mathrm{N}$-iodosuccinimide $(0.645 \mathrm{~g}, 2.87$ $\mathrm{mmol})$ and $\mathrm{TfOH}(0.063 \mathrm{~mL}, 0.72 \mathrm{mmol})$. The mixture was warmed to $0{ }^{\circ} \mathrm{C}$ and continuously stirred at this temperature for $2 \mathrm{~h}$., the reaction mixture was carefully quenched with saturated aqueous $\mathrm{Na}_{2} \mathrm{~S}_{2} \mathrm{O}_{3}$ and then filtered through a short pad of Celite. The filtrate was washed with cold saturated aqueous $\mathrm{NaHCO}_{3}$, brine, dried over $\mathrm{MgSO}_{4}$, filtered, and concentrated in vacuo. The observed yellowish syrup was purified by flash column chromatography on silica gel using ethyl acetate and hexanes $(2: 3, \mathrm{v} / \mathrm{v})$ as the eluent to give $0.712 \mathrm{~g}$ of a colorless syrup compound $\mathbf{2 0}$ in

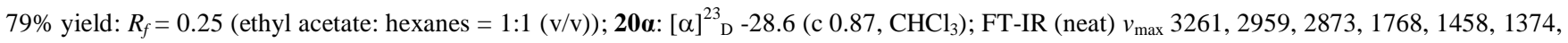
1268, 1227, 1128, 1019, 906, 744, $702 \mathrm{~cm}^{-1}$; ${ }^{1} \mathrm{H}$ NMR (400 MHz, $\left.\mathrm{CDCl}_{3}\right) \delta 7.38-7.28(\mathrm{~m}, 5 \mathrm{H}), 5.28(\mathrm{~s}, 1 \mathrm{H}), 4.61(\mathrm{~s}, 2 \mathrm{H}), 4.50(\mathrm{q}, J=6.6 \mathrm{~Hz}, 1 \mathrm{H})$, $4.36(\mathrm{dd}, J=9.8,1.3 \mathrm{~Hz}, 1 \mathrm{H}), 4.15-4.02(\mathrm{~m}, 8 \mathrm{H}), 3.83(\mathrm{~s}, 3 \mathrm{H}), 3.68(\mathrm{t}, J=10.5 \mathrm{~Hz}, 1 \mathrm{H}), 2.97(\mathrm{dd}, J=12.2,3.5 \mathrm{~Hz}, 1 \mathrm{H}), 2.34(\mathrm{t}, J=12.2 \mathrm{~Hz}, 1 \mathrm{H})$, 1.70-1.61 (m, 4H), 1.49 (s, 3H), 1.44-1.38 (m, 4H), 1.35 (s, 3H), 0.94 (t, $J=7.5 \mathrm{~Hz}, 3 \mathrm{H}), 0.92$ (t, $J=7.4 \mathrm{~Hz}, 3 \mathrm{H})$; HRMS-ESI [M + Na] ${ }^{+}$Calcd for

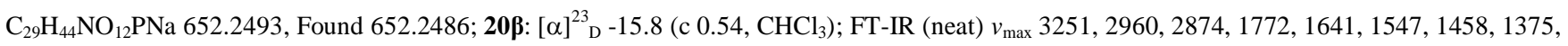
1270, 1083, 1025, 957, 745, $699 \mathrm{~cm}^{-1}$; ${ }^{1} \mathrm{H}$ NMR $\left(400 \mathrm{MHz}, \mathrm{CDCl}_{3}\right) \delta$ 7.36-7.29 (m, 5H), $5.21(\mathrm{~s}, 1 \mathrm{H}), 4.58(\mathrm{~s}, 2 \mathrm{H}), 4.54-4.45(\mathrm{~m}, 2 \mathrm{H}), 4.21(\mathrm{dd}, J$ $=9.9,1.8 \mathrm{~Hz}, 1 \mathrm{H}), 4.17(\mathrm{dd}, J=7.0,1.7 \mathrm{~Hz}, 1 \mathrm{H}), 4.11-4.05(\mathrm{~m}, 4 \mathrm{H}), 3.92,3.85\left(\mathrm{ABX}, J_{\mathrm{AB}}=10.0 \mathrm{~Hz}, J_{\mathrm{AX}}=6.4 \mathrm{~Hz}, J_{\mathrm{BX}}=6.0 \mathrm{~Hz}, 2 \mathrm{H}\right), 3.82(\mathrm{~s}$, $3 \mathrm{H}), 3.64(\mathrm{t}, J=10.5 \mathrm{~Hz}, 1 \mathrm{H}), 2.91(\mathrm{dd}, J=12.8,3.6 \mathrm{~Hz}, 1 \mathrm{H}), 2.39$ (t, $J=12.3 \mathrm{~Hz}, 1 \mathrm{H}), 1.68-1.61(\mathrm{~m}, 4 \mathrm{H}), 1.42(\mathrm{~s}, 3 \mathrm{H}), 1.41-1.35(\mathrm{~m}, 4 \mathrm{H}), 1.35$ $(\mathrm{s}, 3 \mathrm{H}), 0.93(\mathrm{t}, J=7.4 \mathrm{~Hz}, 3 \mathrm{H}), 0.92(\mathrm{t}, J=7.4 \mathrm{~Hz}, 3 \mathrm{H})$; HRMS-ESI $[\mathrm{M}+\mathrm{Na}]^{+}$Calcd for $\mathrm{C}_{29} \mathrm{H}_{44} \mathrm{NO}_{12} \mathrm{PNa} 652.2493$, Found 652.248. 
(D) Synthesis of glucose derivative 17:
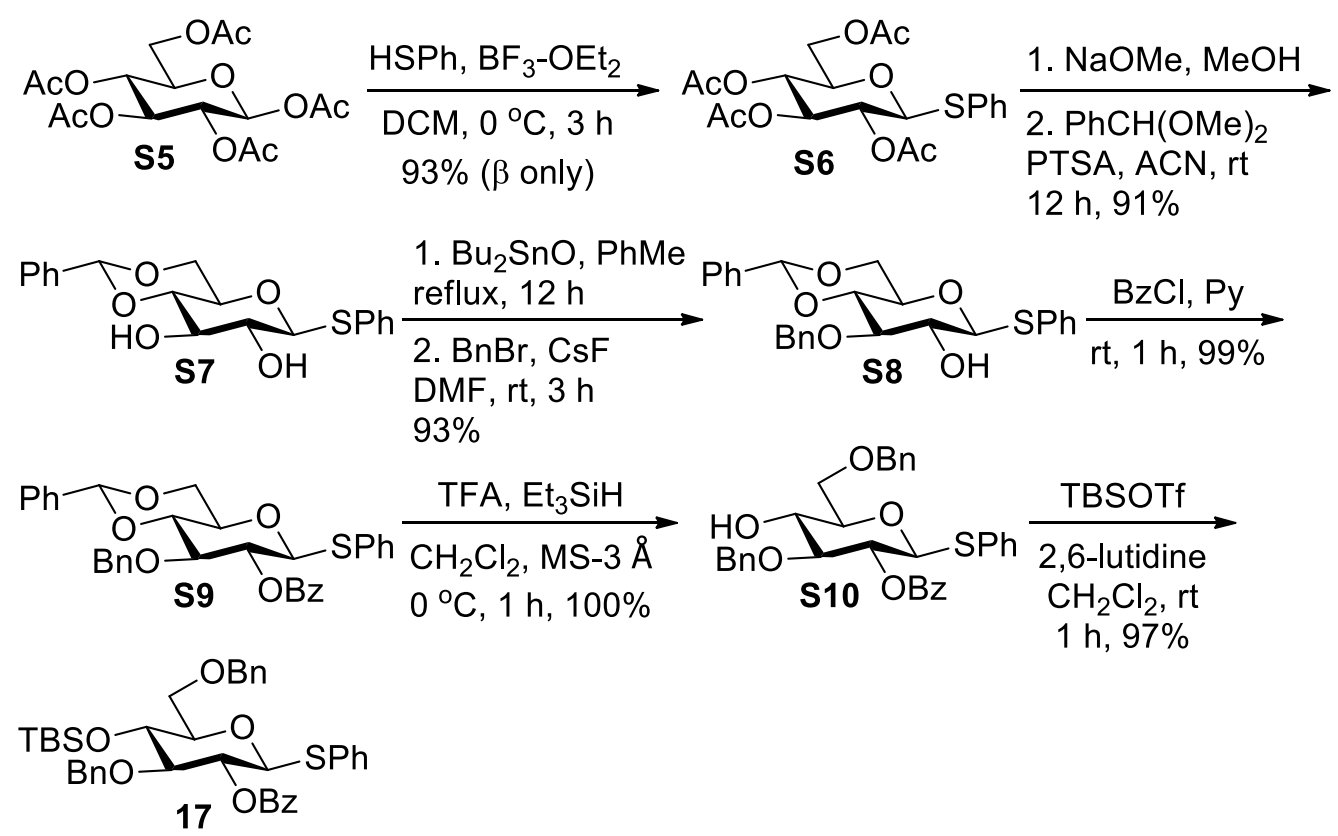

Scheme S3. Synthesis of glucose derivative 17.

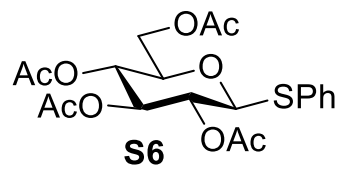

Phenyl 2,3,4,6-tetra- $\boldsymbol{O}$-acetyl-1-thio- $\boldsymbol{\beta}$-D-glucopyranose (S6) To a stirring solution of compound $\mathbf{S 5}$ (4.012 g, $10.26 \mathrm{mmol})$ in $\mathrm{CH}_{2} \mathrm{Cl}_{2}(34 \mathrm{~mL})$ was added boron trifluoride etherate $(3.9 \mathrm{~mL}, 15.38 \mathrm{mmol})$ and thiophenol $(1.5 \mathrm{~mL}, 15.38 \mathrm{mmol})$ at $0{ }^{\circ} \mathrm{C}$. The mixture was continuously stirred at this temperature for $3 \mathrm{~h}$. The mixture was diluted with $\mathrm{CH}_{2} \mathrm{Cl}_{2}$, washed with saturated aqueous $\mathrm{NaHCO}_{3}$, brine, dried over $\mathrm{MgSO}_{4}$, and concentrated in vасио. The resulting yellowish syrup residue was purified by flash column chromatography on silica gel using ethyl acetate and hexanes $(1: 2, \mathrm{v} / \mathrm{v})$ as the eluent to get $4.197 \mathrm{~g}$ of $\mathbf{S 6}$ as a white solid compound in $93 \%$ yield: $R_{f}=0.13$ (ethyl acetate: hexanes $=1: 3(\mathrm{v} / \mathrm{v})$ ); $\mathrm{mp}=$ $119-120{ }^{\circ} \mathrm{C} ;[\alpha]^{23} \mathrm{D}-8.3$ (c 0.23, $\mathrm{CHCl}_{3}$ ); FT-IR (neat) $v_{\max } 3019,2952,2878,1751,1580,1475,1435,1373,1226,1042,910,827,750,694 \mathrm{~cm}^{-1}$; ${ }^{1} \mathrm{H}$ NMR $\left(400 \mathrm{MHz}, \mathrm{CDCl}_{3}\right) \delta$ 7.50-7.48 (m, 2H, ArH), 7.32-7.31 (m, 3H, ArH), $5.22(\mathrm{t}, J=9.8 \mathrm{~Hz}, 1 \mathrm{H}, \mathrm{H}-3), 5.04(\mathrm{t}, J=9.8 \mathrm{~Hz}, 1 \mathrm{H}, \mathrm{H}-4), 4.97(\mathrm{t}$, $J=9.8 \mathrm{~Hz}, 1 \mathrm{H}, \mathrm{H}-2), 4.70(\mathrm{~d}, J=9.8 \mathrm{~Hz}, 1 \mathrm{H}, \mathrm{H}-1), 4.22,4.18\left(\mathrm{ABX}, J_{\mathrm{AB}}=12.4, J_{\mathrm{AX}}=4.9, J_{\mathrm{BX}}=2.2 \mathrm{~Hz}, 2 \mathrm{H}, \mathrm{H}-6 \mathrm{a}, \mathrm{H}-6 \mathrm{~b}\right), 3.72(\mathrm{ddd}, J=9.8,4.9$, $2.2 \mathrm{~Hz}, 1 \mathrm{H}, \mathrm{H}-5), 2.08$ (s, 3H), 2.08 (s, 3H), 2.02 (s, 3H), 1.99 (s, 3H); HRMS-ESI [M + Na] $]^{+}$Calcd for $\mathrm{C}_{20} \mathrm{H}_{24} \mathrm{O}_{9} \mathrm{SNa}$ 463.1033, Found 463.1030.

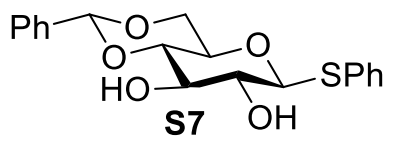

Phenyl 4,6-O-benzylidene-1-thio-p-D-glucopyranoside (S7) MeONa (0.042 g, $0.77 \mathrm{mmol})$ was added to a solution of acetate $\mathbf{S 6}$ (3.405 g, 7.72 $\mathrm{mmol})$ in dry $\mathrm{MeOH}(38 \mathrm{~mL})$ at $0{ }^{\circ} \mathrm{C}$. After stirring for $1 \mathrm{~h}$ at room temperature, the solution was neutralized with Dowex $50 \mathrm{w} \mathrm{X} 8\left[\mathrm{H}^{+}\right]$. The resin was filtered out and washed with $\mathrm{MeOH}$. The filtrate was concentrated under reduced pressure to give a colorless syrup residue $\left(R_{f}=0.30\right.$ $\left(\mathrm{MeOH}: \mathrm{CH}_{2} \mathrm{Cl}_{2}=1: 5(\mathrm{v} / \mathrm{v})\right.$ ). The residue, without further purification, was dissolved in dry acetonitrile $(20 \mathrm{~mL})$ and then benzaldehyde dimethyl acetal $(1.7 \mathrm{~mL}, 11.58 \mathrm{mmol})$ and $p$-toluenesulfonic acid monohydrate $(0.853 \mathrm{~mL}, 3.68 \mathrm{mmol})$ was added to this solution at $0{ }^{\circ} \mathrm{C}$. The mixture was warmed to room temperature and continuously stirred at this temperature for $12 \mathrm{~h}$. The reaction mixture was carefully quenched with triethylamine and extracted with ethyl acetate, washed with cold $1 \mathrm{~N} \mathrm{HCl}$, brine, dried over $\mathrm{MgSO}_{4}$, and concentrated in vacuo. The resulting white solid residue was purified by flash column chromatography on silica gel using ethyl acetate and hexanes $(1: 1, \mathrm{v} / \mathrm{v})$ as the eluent to get $2.529 \mathrm{~g}$ of $\mathbf{S 7}$ as a white solid compound in $91 \%$ yield: $R_{f}=0.48$ (ethyl acetate: hexanes $=2: 1(\mathrm{v} / \mathrm{v})$ ); mp $=186-187{ }^{\circ} \mathrm{C} ;[\alpha]^{23} \mathrm{D}-37.5\left(\mathrm{c} 0.28, \mathrm{CHCl}_{3}\right) ;$ FT-IR $($ neat $) v_{\text {max }}$ 3368, 3312, 2876, 1641, 1582, 1447, 1374, 1074, 1019, 829, 745, $695 \mathrm{~cm}^{-1} ;{ }^{1} \mathrm{H}$ NMR (400 MHz, $\left.\mathrm{CDCl}_{3}\right) \delta$ 7.56-7.53 (m, 2H, ArH), 7.49-7.47 (m, 2H, ArH), 7.37-7.34 (m, 6H, ArH), 5.54 (s, 1H, CHPh), 4.64 (d, J = 9.7 Hz, 1H, H-1), 4.39 (dd, $J=10.5,4.1 \mathrm{~Hz}, 1 \mathrm{H}, \mathrm{H}-6), 3.85(\mathrm{t}, J=8.0 \mathrm{~Hz}, 1 \mathrm{H}$, H-4), 3.81-3.76 (m, 1H, H-6), 3.54-3.51 (m, 2H, H-5, H-3), 3.47 (t, J = 9.7 Hz, 1H, H-2), 2.79 (s, 1H, OH), 2.66 (s, 1H, OH); HRMS-ESI [M + $\mathrm{Na}]^{+}$Calcd for $\mathrm{C}_{19} \mathrm{H}_{20} \mathrm{O}_{5} \mathrm{SNa} 383.0924$, Found 383.0915 . 


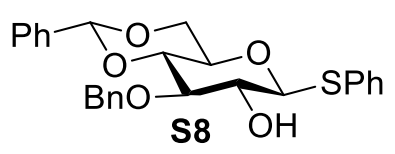

Phenyl 3- $\boldsymbol{O}$-benzyl-4,6- $\boldsymbol{O}$-benzylidene-1-thio- $\boldsymbol{\beta}$-D-glucopyranoside (S8) To a stirring solution of compound $\mathbf{S 7}$ (2.504 g, $6.94 \mathrm{mmol})$ in toluene $(69 \mathrm{~mL})$ was added dibutyltin oxide $(2.594 \mathrm{~g}, 10.42 \mathrm{mmol})$ at $0{ }^{\circ} \mathrm{C}$. The mixture was heated at reflux for $12 \mathrm{~h}$. The reaction mixture was concentrated in vacuo. The resulting mixture, without further purification, was dissolved in dry DMF (43 mL) and then benzyl bromide (2.5 mL, $20.83 \mathrm{mmol})$ and cesium fluoride $(3.167 \mathrm{~g}, 20.83 \mathrm{mmol})$ was added to this solution at $0{ }^{\circ} \mathrm{C}$. The mixture was warmed to room temperature and continuously stirred at this temperature for $3 \mathrm{~h}$. The reaction mixture was extracted with ethyl acetate, washed with cold $1 \mathrm{~N} \mathrm{HCl}$, brine, dried over $\mathrm{MgSO}_{4}$, and concentrated in vacuo. The resulting yellowish syrup residue was purified by flash column chromatography on silica gel using ethyl acetate and $\mathrm{CH}_{2} \mathrm{Cl}_{2}(1: 15, \mathrm{v} / \mathrm{v})$ as the eluent to get $2.925 \mathrm{~g}$ of $\mathbf{S 8}$ as a white solid compound in $93 \%$ yield: $R_{f}=0.30$ (ethyl acetate: hexanes $=1: 3$ $(\mathrm{v} / \mathrm{v})) ; \mathrm{mp}=139-140^{\circ} \mathrm{C} ;[\alpha]^{23} \mathrm{D}-46.8\left(\mathrm{c} 0.52, \mathrm{CHCl}_{3}\right)$; FT-IR (neat) $v_{\max } 3437,3059,2873,1580,1449,1375,1266,1212,1167,1077,1017,920$, 830, 745, $695 \mathrm{~cm}^{-1}$; ${ }^{1} \mathrm{H}$ NMR (400 MHz, $\mathrm{CDCl}_{3}$ ) $\delta$ 7.55-7.53 (m, 2H, ArH), 7.49-7.47 (m, 2H, ArH), 7.41-7.28 (m, 11H, ArH), 5.57 (s, 1H, CHPh), $4.95,4.79(\mathrm{ABq}, J=11.5 \mathrm{~Hz}, 2 \mathrm{H}, \mathrm{CHaHbPh}), 4.64$ (d, $J=9.7 \mathrm{~Hz}, 1 \mathrm{H}, \mathrm{H}-1), 4.39$ (dd, $J=10.4,5.0 \mathrm{~Hz}, 1 \mathrm{H}, \mathrm{H}-6 \mathrm{a}), 3.80$ (t, $J=10.4 \mathrm{~Hz}, 1 \mathrm{H}, \mathrm{H}-6 \mathrm{~b})$, 3.72-3.64 (m, 2H, H-4, H-3), 3.55-3.50 (m, 2H, H-5, H-2), 2.55 (d, J = 2.1 Hz, 1H, OH); HRMS-ESI [M + Na] ${ }^{+}$Calcd for $\mathrm{C}_{26} \mathrm{H}_{26} \mathrm{O}_{5} \mathrm{SNa} 473.1393$, Found 473.1381 .

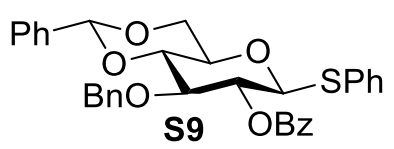

Phenyl 2-O-benzoyl-3-O-benzyl-4,6-O-benzylidene-1-thio-p-D-glucopyranoside (S9) To a stirring solution of compound S8 (2.599 g, 5.54 mmol) in pyridine $(28 \mathrm{~mL})$ was added benzoyl chloride $(0.78 \mathrm{~mL}, 6.65 \mathrm{mmol})$ at $0{ }^{\circ} \mathrm{C}$. The mixture was warmed to room temperature and continuously stirred at this temperature for $1 \mathrm{~h}$. The reaction mixture was carefully quenched with $\mathrm{MeOH}$ and extracted with $\mathrm{CH}_{2} \mathrm{Cl}_{2}$, washed with cold $1 \mathrm{~N} \mathrm{HCl}$, brine, dried over $\mathrm{MgSO}_{4}$, and concentrated in vacuo. The resulting white solid residue was purified by flash column chromatography on silica gel using ethyl acetate and hexanes (1:3, v/v) as the eluent to get $3.039 \mathrm{~g}$ of $\mathbf{S 9}$ as a white solid compound in $99 \%$ yield: $R_{f}=0.38$ (ethyl acetate: hexanes $=1: 3(\mathrm{v} / \mathrm{v})) ; \mathrm{mp}=192-193{ }^{\circ} \mathrm{C} ;[\alpha]^{23}{ }_{\mathrm{D}}+59.8\left(\mathrm{c} 0.27, \mathrm{CHCl}_{3}\right)$; FT-IR (neat) $v_{\max } 3060,2871,1727,1449,1375,1318,1267,1173$, 1093, 999, 919, 840, 745, $701 \mathrm{~cm}^{-1} ;{ }^{1} \mathrm{H}$ NMR $\left(400 \mathrm{MHz}, \mathrm{CDCl}_{3}\right) \delta 8.02(\mathrm{~d}, J=7.5 \mathrm{~Hz}, 2 \mathrm{H}, \mathrm{ArH}), 7.61(\mathrm{t}, J=7.4 \mathrm{~Hz}, 1 \mathrm{H}, \mathrm{ArH}), 7.52-7.38(\mathrm{~m}, 10 \mathrm{H}$, $\mathrm{ArH}), 7.29-7.27(\mathrm{~m}, 3 \mathrm{H}, \mathrm{ArH}), 7.15-7.05(\mathrm{~m}, 5 \mathrm{H}, \mathrm{ArH}), 5.61(\mathrm{~s}, 1 \mathrm{H}, \mathrm{CHPh}), 5.30(\mathrm{dd}, J=10.0,8.8 \mathrm{~Hz}, 1 \mathrm{H}, \mathrm{H}-2), 4.85(\mathrm{~d}, J=10.0 \mathrm{~Hz}, 1 \mathrm{H}, \mathrm{H}-1)$, $4.81,4.67(\mathrm{ABq}, J=11.9 \mathrm{~Hz}, 2 \mathrm{H}, \mathrm{CHaHbPh}), 4.43(\mathrm{dd}, J=10.5,5.0 \mathrm{~Hz}, 1 \mathrm{H}, \mathrm{H}-6 \mathrm{a}), 3.92-3.80$ (m, 3H, H-6b, H-4, H-3), 3.58 (td, $J=9.6,5.0 \mathrm{~Hz}$, 1H, H-5); HRMS-ESI $[\mathrm{M}+\mathrm{Na}]^{+} \mathrm{Calcd}$ for $\mathrm{C}_{33} \mathrm{H}_{30} \mathrm{O}_{6} \mathrm{SNa}$ 577.1655, Found 577.1664.

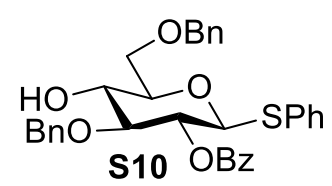

Phenyl 2- $\boldsymbol{O}$-benzoyl-3,6-di- $\boldsymbol{O}$-benzyl-1-thio- $\boldsymbol{\beta}$-D-glucopyranoside (S10) A mixture of the compound $\mathbf{S 9}$ (1.500 g, $2.70 \mathrm{mmol})$ and activated $3 \AA$ powdered molecular sieves $(2.357 \mathrm{~g})$ in anhydrous $\mathrm{CH}_{2} \mathrm{Cl}_{2}(54 \mathrm{~mL})$ was stirred at room temperature for $1 \mathrm{~h}$ under nitrogen to remove any trace amounts of water. The reaction mixture was then cooled to $0{ }^{\circ} \mathrm{C}$ followed by addition of $\mathrm{Et}_{3} \mathrm{SiH}(4.5 \mathrm{~mL}, 27.03 \mathrm{mmol})$ and TFA (2.1 mL, 27.03 mmol). Continuously stirred at this temperature for $1 \mathrm{~h}$, the reaction mixture was carefully quenched with triethylamine and then filtered through a short pad of Celite. The filtrate was washed with cold saturated aqueous $\mathrm{NaHCO}_{3}$, brine, dried over $\mathrm{MgSO}_{4}$, filtered, and concentrated in vacuo. The observed colorless syrup was purified by flash column chromatography on silica gel using ethyl acetate and $\mathrm{CH}_{2} \mathrm{Cl}_{2}(1: 3$, v/v) as the eluent to give $1.499 \mathrm{~g}$ of a white solid $\mathbf{S 1 0}$ in $100 \%$ yield: $R_{f}=0.15$ (ethyl acetate: hexanes $=1: 3(\mathrm{v} / \mathrm{v})$ ); $\mathrm{mp}=90-91{ }^{\circ} \mathrm{C} ;[\alpha]^{23}{ }_{\mathrm{D}}+15.7(\mathrm{c} 0.41, \mathrm{CHCl} 3) ; \mathrm{FT}-\mathrm{IR}$ (neat) $v_{\max } 3452,3206,3062,2918,2858,1723,1591,1448,1360,1270,1122,1071,985,838,745,703 \mathrm{~cm}^{-1} ;{ }^{1} \mathrm{H} \mathrm{NMR}\left(400 \mathrm{MHz}, \mathrm{CDCl}_{3}\right) \delta 8.07$ (d, $J=7.7 \mathrm{~Hz}, 2 \mathrm{H}, \mathrm{ArH}), 7.60(\mathrm{t}, J=7.3 \mathrm{~Hz}, 1 \mathrm{H}, \mathrm{ArH}), 7.49-7.45(\mathrm{~m}, 4 \mathrm{H}, \mathrm{ArH}), 7.36-7.31(\mathrm{~m}, 5 \mathrm{H}, \mathrm{ArH}), 7.25-7.22(\mathrm{~m}, 3 \mathrm{H}, \mathrm{ArH}), 7.18(\mathrm{~s}, 5 \mathrm{H}$, ArH), $5.28(\mathrm{t}, J=9.5 \mathrm{~Hz}, 1 \mathrm{H}, \mathrm{H}-2), 4.82(\mathrm{~d}, J=10.1 \mathrm{~Hz}, 1 \mathrm{H}, \mathrm{H}-1), 4.72,4.67$ (ABq, $J=11.4 \mathrm{~Hz}, 2 \mathrm{H}, \mathrm{CHaHbPh}), 4.61,4.59(\mathrm{ABq}, J=11.8 \mathrm{~Hz}$, 2H, CHaHbPh), 3.86-3.77 (m, 3H, H-6a, H-6b, H-4), 3.71 (t, $J=8.9$ Hz, 1H, H-3), 3.63-3.59 (m, 1H, H-5), 2.75 (bs, 1H, OH); HRMS-ESI [M + $\mathrm{Na}]^{+}$Calcd for $\mathrm{C}_{33} \mathrm{H}_{32} \mathrm{O}_{6} \mathrm{SNa}$ 579.1812, Found 579.1820. 


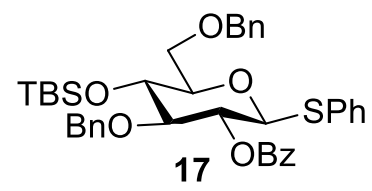

Phenyl 2-O-benzoyl-3,6-di- $\boldsymbol{O}$-benzyl-3- $\boldsymbol{O}$-tert-butyldimethylsilyl-1-thio- $\boldsymbol{\beta}$-D-glucopyranoside (17) To a stirring solution of compound S10 (1.502 g, $2.69 \mathrm{mmol})$ in $\mathrm{CH}_{2} \mathrm{Cl}_{2}(27 \mathrm{~mL})$ was added 2,6-lutidine $(0.96 \mathrm{~mL}, 8.08 \mathrm{mmol})$ and TBSOTf $(0.89 \mathrm{~mL}, 4.04 \mathrm{mmol})$ at $0{ }^{\circ} \mathrm{C}$. The mixture was warmed to room temperature and continuously stirred at this temperature for $1 \mathrm{~h}$. The reaction mixture was carefully quenched with $\mathrm{MeOH}$ and extracted with ethyl acetate, washed with cold $1 \mathrm{~N} \mathrm{HCl}$, brine, dried over $\mathrm{MgSO}_{4}$, and concentrated in vacuo. The resulting colorless syrup residue was purified by flash column chromatography on silica gel using $\mathrm{CH}_{2} \mathrm{Cl}_{2}$ and hexanes $(1: 1$, v/v) as the eluent to get $1.756 \mathrm{~g}$ of 17 as a white solid compound in $97 \%$ yield: $R_{f}=0.18\left(\mathrm{CH}_{2} \mathrm{Cl}_{2}\right.$ : hexanes $\left.=1: 1(\mathrm{v} / \mathrm{v})\right) ; \mathrm{mp}=65-66{ }^{\circ} \mathrm{C} ;[\alpha]^{23}{ }_{\mathrm{D}}+60.1(\mathrm{c} 0.57, \mathrm{CHCl} 3)$; FT-IR (neat) $v_{\max } 3061$, 3033, 2934, 2860, 1729, 1593, 1461, 1361, 1321, 1261, 1096, 1035, 994, 841, 775, 742, $702 \mathrm{~cm}^{-1}$; ${ }^{1} \mathrm{H}$ NMR $\left(400 \mathrm{MHz}, \mathrm{CDCl}_{3}\right) \delta 7.99(\mathrm{~d}, J=7.8$ $\mathrm{Hz}, 2 \mathrm{H}, \mathrm{ArH}), 7.55$ (t, $J=7.4 \mathrm{~Hz}, 1 \mathrm{H}, \mathrm{ArH}), 7.49$ (d, $J=7.4 \mathrm{~Hz}, 2 \mathrm{H}, \mathrm{ArH}), 7.41(\mathrm{t}, J=7.7 \mathrm{~Hz}, 2 \mathrm{H}, \mathrm{ArH}), 7.37-7.29(\mathrm{~m}, 5 \mathrm{H}, \mathrm{ArH}), 7.22-7.17(\mathrm{~m}$, $3 \mathrm{H}, \mathrm{ArH}), 7.11(\mathrm{~s}, 5 \mathrm{H}, \mathrm{ArH}), 5.32(\mathrm{t}, J=10.0 \mathrm{~Hz}, 1 \mathrm{H}, \mathrm{H}-2), 4.85$ (d, $J=10.0 \mathrm{~Hz}, 1 \mathrm{H}, \mathrm{H}-1), 4.65\left(\mathrm{~s}, 2 \mathrm{H}, \mathrm{CH}_{2} \mathrm{Ph}\right), 4.66,4.55(\mathrm{ABq}, J=11.4 \mathrm{~Hz}, 2 \mathrm{H}$, $\mathrm{CHaHbPh}), 3.86-3.60$ (m, 5H, H-6, H-5, H-4, H-3), 0.87 (s, 9H, $\left.\left(\mathrm{CH}_{3}\right)_{3} \mathrm{C}\right), 0.02$ (s, 3H, $\left.\mathrm{CH}_{3} \mathrm{Si}\right),-0.03\left(\mathrm{~s}, 3 \mathrm{H}, \mathrm{CH}_{3} \mathrm{Si}\right) ;{ }^{13} \mathrm{C} \mathrm{NMR}(100 \mathrm{MHz}, \mathrm{CDCl}$ )

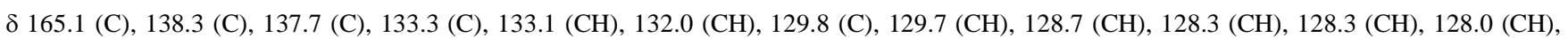
$127.5(\mathrm{CH}), 127.3(\mathrm{CH}), 86.1(\mathrm{CH}), 84.7(\mathrm{CH}), 80.7(\mathrm{CH}), 75.1\left(\mathrm{CH}_{2}\right), 73.3\left(\mathrm{CH}_{2}\right), 72.8(\mathrm{CH}), 70.9(\mathrm{CH}), 69.3\left(\mathrm{CH}_{2}\right), 25.9\left(\mathrm{CH}_{3}\right), 17.9(\mathrm{C}),-3.8$ $\left(\mathrm{CH}_{3}\right),-4.8\left(\mathrm{CH}_{3}\right)$; HRMS-ESI [M + Na] ${ }^{+}$Calcd for $\mathrm{C}_{39} \mathrm{H}_{46} \mathrm{O}_{6} \mathrm{SSiNa}$ 693.2677, Found 693.2677.

(E) Synthesis of galactose derivative 21:
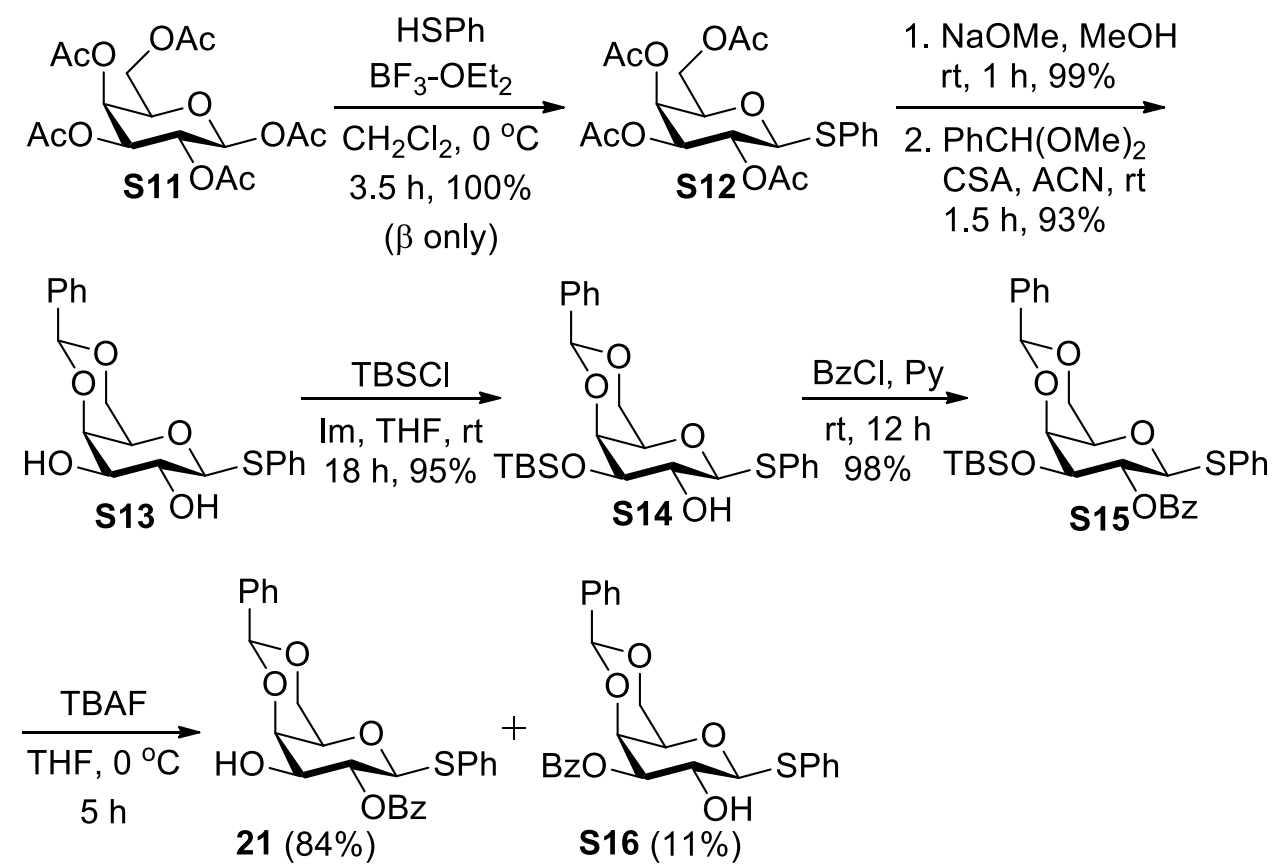

Scheme S4. Synthesis of galactose derivative 21.

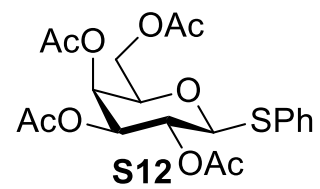

Phenyl 2,3,4,6-tetra-O -acetyl-1-thio- $\boldsymbol{\beta}$-D-galactopyranoside (S12) To a stirring solution of $\mathbf{S 1 1}(10.102 \mathrm{~g}, 25.64 \mathrm{mmol})$ in $\mathrm{CH}_{2} \mathrm{Cl} \mathrm{L}_{2}(85 \mathrm{~mL})$ was added boron trifluoride etherate $(9.6 \mathrm{~mL}, 38.46 \mathrm{mmol})$ and thiophenol $(3.8 \mathrm{~mL}, 38.46 \mathrm{mmol})$ at $0{ }^{\circ} \mathrm{C}$. The mixture was continuously stirred at this temperature for $3 \mathrm{~h}$. The mixture was diluted with $\mathrm{CH}_{2} \mathrm{Cl}_{2}$, washed with saturated aqueous $\mathrm{NaHCO}_{3}$, brine, dried over $\mathrm{MgSO}_{4}$, and concentrated in vacuo. The resulting yellowish syrup was purified by flash column chromatography on silica gel using ethyl acetate and hexanes (1:3, v/v) as the eluent to get $11.264 \mathrm{~g}$ of $\mathbf{S 1 2}$ as a white solid compound in $100 \%$ yield: $R_{f}=0.43($ ethyl acetate: hexanes $=1: 1(\mathrm{v} / \mathrm{v})) ;[\alpha]^{24}{ }_{\mathrm{D}}+7.5\left(\mathrm{c} 0.74, \mathrm{CHCl}_{3}\right)$; FT-IR (neat) $v_{\max } 3020,2970,2874,1749,1645,1581,1474,1435,1372,1228,1148,1052,951,913,, 752,698 \mathrm{~cm}^{-1} ;{ }^{1} \mathrm{H} \mathrm{NMR}(400 \mathrm{MHz}$, 
$\left.\mathrm{CDCl}_{3}\right) \delta$ 7.52-7.50 (m, 2H, ArH), 7.32-7.30 (m, 3H, ArH), 5.41 (d, J=3.3 Hz, 1H, H-4), 5.24 (t, J=10.0 Hz, 1H, H-2), 5.04 (dd, $J=10.0,3.3 \mathrm{~Hz}$, $1 \mathrm{H}, \mathrm{H}-3), 4.71(\mathrm{~d}, J=10.0 \mathrm{~Hz}, 1 \mathrm{H}, \mathrm{H}-1), 4.18,4.11\left(\mathrm{ABX}, J_{\mathrm{AB}}=11.3 \mathrm{~Hz}, J_{\mathrm{AX}}=7.1 \mathrm{~Hz}, J_{\mathrm{BX}}=6.2 \mathrm{~Hz}, 2 \mathrm{H}, \mathrm{H}-6 \mathrm{a}, \mathrm{H}-6 \mathrm{~b}\right), 3.95-3.92(\mathrm{~m}, 1 \mathrm{H}, \mathrm{H}-5)$, 2.12 (s, 3H, Ac), 2.09 (s, 3H, Ac), 2.04 (s, 3H, Ac), 1.97 (s, 3H, Ac); HRMS-ESI [M + Na] $]^{+}$Calcd for $\mathrm{C}_{20} \mathrm{H}_{24} \mathrm{O}_{9} \mathrm{SNa}$ 463.1033, Found 463.1023.

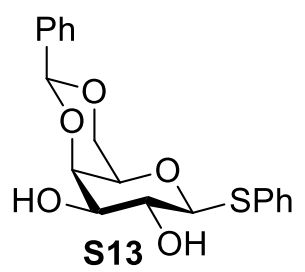

Phenyl 4,6-O-benzylidene-1-thio- $\boldsymbol{\beta}$-D-galactopyranoside (S13) MeONa (0.040 g, $0.74 \mathrm{mmol})$ was added to a solution of acetate S12 (3.235 g, $7.35 \mathrm{mmol})$ in dry $\mathrm{MeOH}(25 \mathrm{~mL})$ at $0{ }^{\circ} \mathrm{C}$. After stirring for $1 \mathrm{~h}$ at room temperature, the solution was neutralized with Dowex $50 \mathrm{w} \mathrm{X} 8\left[\mathrm{H}^{+}\right]$. The resin was filtered out and washed with $\mathrm{MeOH}$. The filtrate was concentrated under reduced pressure to give a colorless syrup residue $\left(R_{f}=0.30\right.$ (MeOH: $\mathrm{CH}_{2} \mathrm{Cl}_{2}=1: 5(\mathrm{v} / \mathrm{v})$ ). The residue, without further purification, was dissolved in dry acetonitrile $(37 \mathrm{~mL})$ and then benzaldehyde dimethyl acetal $(2.2 \mathrm{~mL}, 14.70 \mathrm{mmol})$ and $\mathrm{CSA}(0.853 \mathrm{~mL}, 3.68 \mathrm{mmol})$ was added to this solution at $0{ }^{\circ} \mathrm{C}$. The mixture was warmed to room temperature and continuously stirred at this temperature for $1.5 \mathrm{~h}$. The reaction mixture was carefully quenched with triethylamine and extracted with ethyl acetate, washed with cold $1 \mathrm{~N} \mathrm{HCl}$, brine, dried over $\mathrm{MgSO}_{4}$, and concentrated in vacuo. The resulting white solid residue was purified by flash column chromatography on silica gel using ethyl acetate and hexanes $(3: 1, \mathrm{v} / \mathrm{v})$ as the eluent to get $2.457 \mathrm{~g}$ of $\mathbf{S 1 3}$ as a white solid compound in 93\% yield: $R_{f}=0.18$ (ethyl acetate: hexanes $=3: 1(\mathrm{v} / \mathrm{v})$ ); $\mathrm{mp}=154-155{ }^{\circ} \mathrm{C} ;[\alpha]^{24} \mathrm{D}-32.8\left(\mathrm{c} 0.33, \mathrm{CHCl}_{3}\right) ;$ FT-IR (neat) $v_{\max } 3392,3062,2868,1446$, 1401, 1358, 1241, 1163, 1071, 1034, 994, 895, 860, 812, 745, $695 \mathrm{~cm}^{-1}$; ${ }^{1} \mathrm{H}$ NMR (400 MHz, $\left.\mathrm{CDCl}_{3}\right) \delta 7.69$ (dd, $\left.J=7.6,0.8 \mathrm{~Hz}, 2 \mathrm{H}, \mathrm{ArH}\right), 7.41-$ 7.29 (m, 8H, ArH), 5.52 (s, 1H, CHPh), 4.54-4.49 (m, 1H, H-2), 4.39 (dd, $J=12.5,0.8$ Hz, 1H, H-6a), 4.22 (s, 1H, H-4), 4.04 (dd, $J=12.5,1.3$ Hz, 1H, H-6b), 3.70-3.66 (m, 2H, H-3, H-1), 3.57 (s, 1H, H-5), 2.58 (s, 1H, OH), 2.56 (d, $J=7.5 \mathrm{~Hz}, 1 \mathrm{H}, \mathrm{OH})$; HRMS-ESI [M + Na] $]^{+}$Calcd for $\mathrm{C}_{19} \mathrm{H}_{20} \mathrm{O}_{5} \mathrm{SNa} 383.0924$, Found 383.0921.

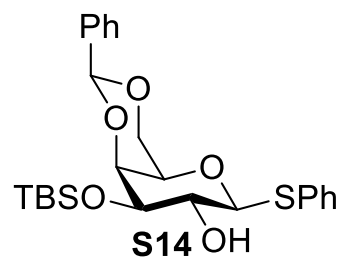

Phenyl 4,6-O-benzylidene-3-O-tert-butyldimethylsilyl-1-thio- $\boldsymbol{\beta}$-D-galactopyranoside (S14) To a stirring solution of compound S13 (1.902 g, $5.28 \mathrm{mmol})$ and imidazole $(1.794 \mathrm{~g}, 26.39 \mathrm{mmol})$ in THF $(26 \mathrm{~mL})$ was added tert-butyldimethylchlorosilane $(0.956 \mathrm{~g}, 6.33 \mathrm{mmol})$ at $0{ }^{\circ} \mathrm{C}$. The mixture was warmed to room temperature and continuously stirred at this temperature for $18 \mathrm{~h}$. The reaction mixture was carefully quenched with $\mathrm{MeOH}$ and extracted with ethyl acetate, washed with cold $1 \mathrm{~N} \mathrm{HCl}$, brine, dried over $\mathrm{MgSO}_{4}$, and concentrated in vacuo. The resulting colorless syrup residue was purified by flash column chromatography on silica gel using ethyl acetate and hexanes $(1: 8, \mathrm{v} / \mathrm{v})$ as the eluent to get $2.385 \mathrm{~g}$ of S14 as a colorless syrup compound in $95 \%$ yield: $R_{f}=0.33$ (ethyl acetate: hexanes $=1: 3(\mathrm{v} / \mathrm{v})$ ); $[\alpha]^{24} \mathrm{D}-11.5\left(\mathrm{c} 0.30, \mathrm{CHCl}_{3}\right)$; FT-IR (neat) $v_{\max }$ $3493,3060,2933,2858,1468,1396,1362,1250,1136,1085,1036,913,878,838,775,742,694 \mathrm{~cm}^{-1} ;{ }^{1} \mathrm{H} \mathrm{NMR}(400 \mathrm{MHz}, \mathrm{CDCl})_{3} \delta 7.67(\mathrm{dd}, J$ $=8.1,1.3 \mathrm{~Hz}, 2 \mathrm{H}, \mathrm{ArH}), 7.48-7.45(\mathrm{~m}, 2 \mathrm{H}, \mathrm{ArH}), 7.38-7.36(\mathrm{~m}, 3 \mathrm{H}, \mathrm{ArH}), 7.24-7.19(\mathrm{~m}, 3 \mathrm{H}, \mathrm{ArH}), 5.50(\mathrm{~s}, 1 \mathrm{H}, \mathrm{CHPh}), 4.56(\mathrm{~d}, J=9.4 \mathrm{~Hz}, 1 \mathrm{H}$, H-1), 4.39 (dd, $J=12.4,1.2 \mathrm{~Hz}, 1 \mathrm{H}, \mathrm{H}-6 \mathrm{a}), 4.06$ (d, $J=3.3 \mathrm{~Hz}, 1 \mathrm{H}, \mathrm{H}-4), 4.02$ (dd, $J=12.4,1.4 \mathrm{~Hz}, 1 \mathrm{H}, \mathrm{H}-6 \mathrm{~b}), 3.84$ (t, $J=9.4 \mathrm{~Hz}, 1 \mathrm{H}, \mathrm{H}-2), 3.74$ (dd, $J=9.4,3.3 \mathrm{~Hz}, 1 \mathrm{H}, \mathrm{H}-3), 3.51$ (s, 1H, H-5), 2.36 (bs, 1H, OH), 0.89 (s, 9H, $\left.\left(\mathrm{CH}_{3}\right)_{3} \mathrm{C}\right), 0.10$ (s, 3H, $\left.\mathrm{CH}_{3} \mathrm{Si}\right), 0.09$ (s, 3H, CH $3 \mathrm{Si}$ ); HRMS-ESI $[\mathrm{M}+\mathrm{Na}]^{+}$Calcd for $\mathrm{C}_{25} \mathrm{H}_{34} \mathrm{O}_{5} \mathrm{SSiNa} 497.1788$, Found 497.1790.

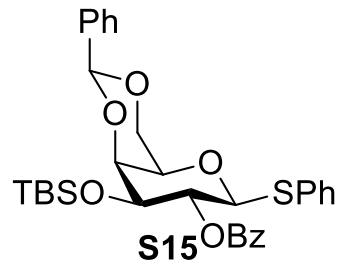

Phenyl 2- $\boldsymbol{O}$-benzoyl-4,6- $\boldsymbol{O}$-benzylidene-3- $\boldsymbol{O}$-tert-butyldimethylsilyl-1-thio- $\boldsymbol{\beta}$-D-galactopyranoside (S15) To a stirring solution of compound S14 $(2.101 \mathrm{~g}, 4.42 \mathrm{mmol})$ in pyridine $(44 \mathrm{~mL})$ was added benzoyl chloride $(0.62 \mathrm{~mL}, 5.31 \mathrm{mmol})$ at $0{ }^{\circ} \mathrm{C}$. The mixture was warmed to room temperature and continuously stirred at this temperature for $12 \mathrm{~h}$. The reaction mixture was carefully quenched with $\mathrm{MeOH}$ and extracted with 
ethyl acetate, washed with cold $1 \mathrm{~N} \mathrm{HCl}$, brine, dried over $\mathrm{MgSO}_{4}$, and concentrated in vacuo. The resulting white solid residue was purified by flash column chromatography on silica gel using ethyl acetate and hexanes $(1: 3, \mathrm{v} / \mathrm{v})$ as the eluent to get $2.515 \mathrm{~g}$ of $\mathbf{S 1 5}$ as a white solid compound in $98 \%$ yield: $R_{f}=0.28$ (ethyl acetate: hexanes $=1: 3(\mathrm{v} / \mathrm{v})$ ); $\mathrm{mp}=194-195{ }^{\circ} \mathrm{C} ;[\alpha]^{24} \mathrm{D}+6.3\left(\mathrm{c} 0.37, \mathrm{CHCl}_{3}\right) ;$ FT-IR (neat) $v_{\max } 3063,2935,2859$, 1727, 1593, 1451, 1397, 1358, 1260, 1145, 1102, 1031, 999, 914, 884, 840, 749, $702 \mathrm{~cm}^{-1} ;{ }^{1} \mathrm{H}$ NMR (400 MHz, CDCl 3$) \delta 8.04(\mathrm{dd}, J=8.3,1.0 \mathrm{~Hz}$, 2H, ArH), 7.59-7.17 (m, 13H, ArH), $5.52(\mathrm{~s}, 1 \mathrm{H}, \mathrm{CHPh}), 5.49$ (t, $J=9.8 \mathrm{~Hz}, \mathrm{H}-2), 4.83(\mathrm{~d}, J=9.8 \mathrm{~Hz}, 1 \mathrm{H}, \mathrm{H}-1), 4.43(\mathrm{dd}, J=12.4,1.3 \mathrm{~Hz}, 1 \mathrm{H}, \mathrm{H}-$ 6a), 4.12 (d, $J=2.8 \mathrm{~Hz}, 1 \mathrm{H}, \mathrm{H}-4), 4.07$ (dd, $J=12.4,1.4 \mathrm{~Hz}, 1 \mathrm{H}, \mathrm{H}-6 \mathrm{~b}), 4.03$ (dd, $J=9.8,2.8 \mathrm{~Hz}, 1 \mathrm{H}, \mathrm{H}-3), 3.58$ (s, 1H, H-5), 0.72 (s, $9 \mathrm{H}$, $\left.\left(\mathrm{CH}_{3}\right)_{3} \mathrm{C}\right), 0.01$ (s, 3H, $\left.\mathrm{CH}_{3} \mathrm{Si}\right),-0.15$ (s, 3H, $\mathrm{CH}_{3} \mathrm{Si}$ ); HRMS-ESI [M + Na] $]^{+}$Calcd for $\mathrm{C}_{32} \mathrm{H}_{38} \mathrm{O}_{6} \mathrm{SSiNa}$ 601.2051, Found 601.2054.
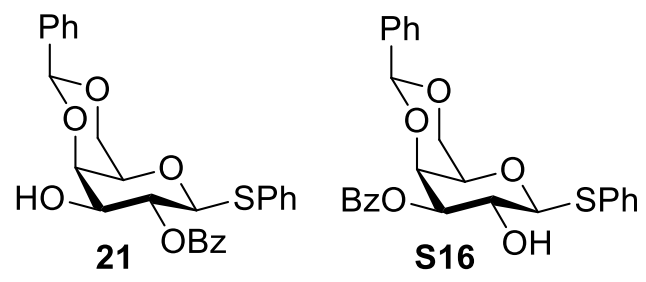

Phenyl 2- $O$-benzoyl-4,6- $O$-benzylidene-1-thio- $\beta$-D-galactopyranoside $\quad(21)$ and phenyl $3-O$-benzoyl-4,6- $O$-benzylidene-1-thio- $\beta$-Dgalactopyranoside (S16) To a stirring solution of compound $\mathbf{S 1 5}(2.000 \mathrm{~g}, 3.45 \mathrm{mmol})$ in THF (17 mL) was added tetra- $n$-butylammonium fluoride $(7.7 \mathrm{~mL})$ at $0{ }^{\circ} \mathrm{C}$. The mixture was continuously stirred at this temperature for $5 \mathrm{~h}$. The reaction mixture was extracted with ethyl acetate, washed with brine, dried over $\mathrm{MgSO}_{4}$, and concentrated in vacuo. The resulting colorless syrup residue was purified by flash column chromatography on silica gel using ethyl acetate and hexanes $(1: 2, \mathrm{v} / \mathrm{v})$ then ethyl acetate and hexanes $(2: 1, \mathrm{v} / \mathrm{v})$ as the eluent to get $1.351 \mathrm{~g}$ of $\mathbf{2 1} \mathrm{as}$ a white solid compound in $84 \%$ yield and $0.177 \mathrm{~g}$ of $\mathbf{S 1 6}$ as a white solid compound in $11 \%$ yield: $\mathbf{2 1}: R_{f}=0.10$ (ethyl acetate: hexanes $=1: 2$ $(\mathrm{v} / \mathrm{v})) ; \mathrm{mp}=179-180^{\circ} \mathrm{C} ;[\alpha]^{24}{ }_{\mathrm{D}}-34.0\left(\mathrm{c} 0.50, \mathrm{CHCl}_{3}\right)$; FT-IR (neat) $v_{\max } 3453,3063,2868,1721,1592,1447,1401,1363,1263,1168,1095,1028$, 994, 898, 874, 812, 751, $704 \mathrm{~cm}^{-1}$; ${ }^{1} \mathrm{H}$ NMR $\left(400 \mathrm{MHz}, \mathrm{CDCl}_{3}\right) \delta 8.07(\mathrm{dd}, J=8.2,1.0 \mathrm{~Hz}, 2 \mathrm{H}, \mathrm{ArH}), 7.61-7.25(\mathrm{~m}, 13 \mathrm{H}, \mathrm{ArH}), 5.55(\mathrm{~s}, 1 \mathrm{H}$, $\mathrm{CHPh}$ ), 5.26 (t, $J=9.8 \mathrm{~Hz}, 1 \mathrm{H}, \mathrm{H}-2), 4.83$ (d, $J=9.8 \mathrm{~Hz}, 1 \mathrm{H}, \mathrm{H}-1), 4.44$ (dd, $J=12.4,1.2 \mathrm{~Hz}, 1 \mathrm{H}, \mathrm{H}-6 \mathrm{a}), 4.28$ (dd, $J=3.6,0.6 \mathrm{~Hz}, 1 \mathrm{H}, \mathrm{H}-4), 4.08$ (dd, $J=12.4,1.5 \mathrm{~Hz}, 1 \mathrm{H}, \mathrm{H}-6 \mathrm{~b}$ ), 3.91 (bs, $1 \mathrm{H}, \mathrm{H}-3$ ), 3.63 (s, 1H, H-5), 2.60 (bs, 1H, OH); HRMS-ESI [M + Na] $]^{+} \mathrm{Calcd}$ for $\mathrm{C}_{26} \mathrm{H}_{24} \mathrm{O}_{6} \mathrm{SNa} 487.1186$, Found 487.1188; S16: $R_{f}=0.23$ (ethyl acetate: hexanes $=1: 2(\mathrm{v} / \mathrm{v})$ ); $\mathrm{mp}=173-174{ }^{\circ} \mathrm{C} ;[\alpha]^{23}{ }_{\mathrm{D}}+36.5\left(\mathrm{c} 0.87, \mathrm{CHCl}_{3}\right)$; FT-IR (neat) $v_{\max } 3482,3062$, 2867, 1712, 1592, 1447, 1400, 1362, 1278, 1171, 1096, 1036, 1000, 896, 751, $706 \mathrm{~cm}^{-1} ;{ }^{1} \mathrm{H}$ NMR $\left(400 \mathrm{MHz}, \mathrm{CDCl}_{3}\right) \delta 8.04(\mathrm{~d}, J=7.5 \mathrm{~Hz}, 2 \mathrm{H}$, $\mathrm{ArH}), 7.71(\mathrm{~d}, J=7.5 \mathrm{~Hz}, 2 \mathrm{H}, \mathrm{ArH}), 7.54(\mathrm{t}, J=7.4 \mathrm{~Hz}, 1 \mathrm{H}, \mathrm{ArH}), 7.42-7.25(\mathrm{~m}, 10 \mathrm{H}, \mathrm{ArH}), 5.50(\mathrm{~s}, 1 \mathrm{H}, \mathrm{CHPh}), 5.19(\mathrm{dd}, J=9.5,3.3 \mathrm{~Hz}, 1 \mathrm{H}, \mathrm{H}-$ 3), 4.68 (d, $J=9.5 \mathrm{~Hz}, 1 \mathrm{H}, \mathrm{H}-1), 4.50$ (d, $J=3.3 \mathrm{~Hz}, 1 \mathrm{H}, \mathrm{H}-4), 4.41$ (dd, $J=12.4,0.9 \mathrm{~Hz}, 1 \mathrm{H}, \mathrm{H}-6 \mathrm{a}), 4.16(\mathrm{t}, J=9.5 \mathrm{~Hz}, 1 \mathrm{H}, \mathrm{H}-2), 4.06$ (dd, $J=$ 12.4, $0.9 \mathrm{~Hz}, 1 \mathrm{H}, \mathrm{H}-6 \mathrm{~b}), 3.69$ (s, 1H, H-5), 2.44 (s, OH); HRMS-ESI [M + Na] $]^{+}$Calcd for $\mathrm{C}_{26} \mathrm{H}_{24} \mathrm{O}_{6} \mathrm{SNa}$ 487.1186, Found 487.1177. 
(F) Synthesis of the protected phytoceramide 16:
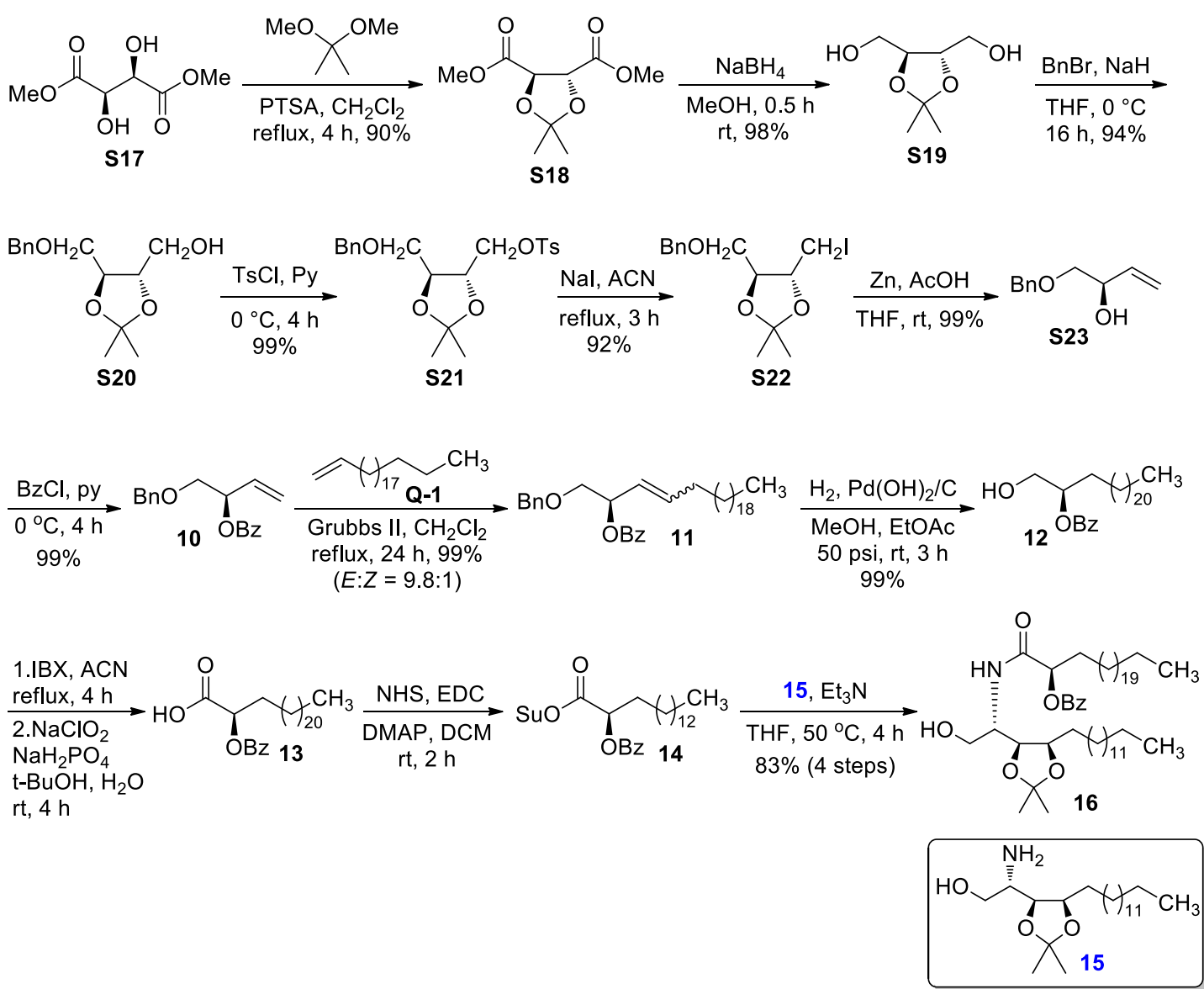

Scheme S5. Synthesis of the protected phytoceramide $\mathbf{1 6 .}$<smiles>COC(=O)[C@H]1OC(C)(C)O[C@H]1C(=O)OC</smiles>

S18

(4R,5R)-2,2-Dimethyl-1,3-dioxolane-4,5-dicarboxylate (S18) To a stirring solution of $\mathbf{S 1 7}(0.100 \mathrm{~g}, 0.56 \mathrm{mmol})$ in $\mathrm{CH}_{2} \mathrm{Cl}_{2}(1.4 \mathrm{~mL})$ was added 2,2-dimethoxypropane $(0.4 \mathrm{~mL}, 3.36 \mathrm{mmol})$ and $p$-toluenesulfonic acid monohydrate $(0.008 \mathrm{~g}, 0.04 \mathrm{mmol})$ at $0{ }^{\circ} \mathrm{C}$. The mixture was heated at reflux for $4 \mathrm{~h}$. The reaction mixture was washed with cold saturated aqueous $\mathrm{NaHCO}_{3}$, brine, dried over $\mathrm{MgSO}_{4}$, and concentrated in vacuo. The observed brown liquid was purified by flash column chromatography on silica gel using ethyl acetate and hexanes (1:6, v/v) as the eluent to give $0.108 \mathrm{~g}$ of a colorless liquid S18 in 90\% yield: $R_{f}=0.30$ (ethyl acetate:hexanes $=1: 4(\mathrm{v} / \mathrm{v})$ ); $[\alpha]^{25}-0.1\left(\mathrm{c} 2.00, \mathrm{CHCl}_{3}\right)$; FT-IR (neat) $v_{\text {max }} 3448$, 2592, 1752, 1442, 1378, 1213, 1107, 1014, 866, $703 \mathrm{~cm}^{-1} ;{ }^{1} \mathrm{H}$ NMR (400 MHz, $\left.\mathrm{CDCl}_{3}\right) \delta 4.81(\mathrm{~s}, 2 \mathrm{H}), 3.83\left(\mathrm{~s}, 6 \mathrm{H},-\mathrm{OCH}_{3}\right), 1.50\left(\mathrm{~s}, 6 \mathrm{H},-\mathrm{CH}_{3}\right)$; HRMS-ESI $[\mathrm{M}+\mathrm{Na}]^{+}$Calcd for $\mathrm{C}_{9} \mathrm{H}_{14} \mathrm{O}_{6} \mathrm{Na} 241.0683$, Found 241.0699 .

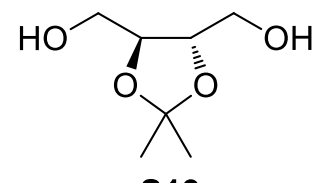

\section{S19}

(4S,5S)-4,5-Dihydroxymethyl-2,2-dimethyl-1,3-dioxolan (S19) To a stirring solution of $\mathbf{S 1 8}(1.986 \mathrm{~g}, 9.11 \mathrm{mmol})$ in $\mathrm{MeOH}(18 \mathrm{~mL})$ was added sodium borohydride $(1.034 \mathrm{~g}, 27.32 \mathrm{mmol})$ at $0{ }^{\circ} \mathrm{C}$. The mixture was warmed to room temperature and continuously stirred at this temperature for $0.5 \mathrm{~h}$. The reaction mixture was washed with brine, dried over $\mathrm{MgSO}_{4}$, and concentrated in vacuo. The resulting colorless liquid residue was purified by flash column chromatography on silica gel using ethyl acetate as the eluent to get $1.447 \mathrm{~g}$ of $\mathbf{S 1 9}$ as a colorless liquid compound in 
98\% yield: $R_{f}=0.13$ (ethyl acetate: hexanes $=1: 1(\mathrm{v} / \mathrm{v})$ ); $[\alpha]^{25}{ }_{\mathrm{D}}-18.0\left(\mathrm{c} 0.31, \mathrm{CHCl}_{3}\right) ;{ }^{1} \mathrm{H} \mathrm{NMR}\left(400 \mathrm{MHz}, \mathrm{CDCl}_{3}\right) \delta 4.04(\mathrm{~s}, 2 \mathrm{H}), 3.84-3.69(\mathrm{~m}$, $\left.4 \mathrm{H},-\mathrm{CH}_{2} \mathrm{OH}\right), 2.03(\mathrm{bs}, 1 \mathrm{H},-\mathrm{OH}), 1.44\left(\mathrm{~s}, 6 \mathrm{H},-\mathrm{CH}_{3}\right)$; LRMS-ESI [M + Na] $]^{+}$Calcd for $\mathrm{C}_{7} \mathrm{H}_{14} \mathrm{O}_{4} \mathrm{Na}$ 185.1, Found 185.1.

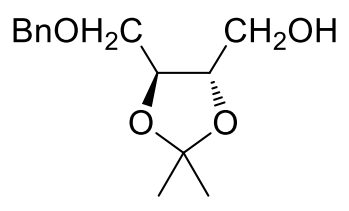

S20

(4S,5S)-4-Benzyloxymethyl-5-Hydroxymethyl-2,2-dimethyl-1,3-dioxolan (S20) To a stirring solution of compound S19 (5.993 g, $36.97 \mathrm{mmol})$ in THF $(184 \mathrm{~mL})$ was added sodium hydride $(1.62 \mathrm{~g}, 40.67 \mathrm{mmol})$ at $0{ }^{\circ} \mathrm{C}$. After stirring for 30 minutes at $0{ }^{\circ} \mathrm{C}$, benzyl bromide $(4.7 \mathrm{~mL}, 1.43$ $\mathrm{g} / \mathrm{mL}, 40.67 \mathrm{mmol}$ ) was added to the reaction mixture and continuously stirred at this temperature for $16 \mathrm{~h}$. The reaction mixture was extracted with ethyl acetate, washed with brine, dried over $\mathrm{MgSO}_{4}$, and concentrated in vacuo. The resulting yellowish liquid residue was purified by flash column chromatography on silica gel using ethyl acetate and hexanes $(1: 6, \mathrm{v} / \mathrm{v})$ as the eluent to get $8.763 \mathrm{~g}$ of $\mathbf{S 2 0}$ as a colorless syrup compound in 94\% yield: $R_{f}=0.40$ (ethyl acetate: hexanes $=1: 1(\mathrm{v} / \mathrm{v})$ ); $[\alpha]^{25}+39.7\left(\mathrm{c} 2.00, \mathrm{CHCl}_{3}\right)$; FT-IR (neat) $v_{\max } 3427,2869,2360,1374,1217,1081$, 847, $741 \mathrm{~cm}^{-1}$; ${ }^{1} \mathrm{H}$ NMR $\left(400 \mathrm{MHz}, \mathrm{CDCl}_{3}\right) \delta$ 7.37-7.30 (m, 5H, ArH), 4.59 (s, 2H, -OCH $\left.\mathrm{Ar}\right), 4.08-3.92(\mathrm{~m}, 2 \mathrm{H}), 3.80-3.54\left(\mathrm{~m}, 4 \mathrm{H},-\mathrm{CH}_{2} \mathrm{OH},-\right.$ $\mathrm{CH}_{2} \mathrm{OBn}$ ), $2.22(\mathrm{bs}, 1 \mathrm{H},-\mathrm{OH}) 1.42\left(\mathrm{~s}, 3 \mathrm{H},-\mathrm{CH}_{3}\right), 1.42\left(\mathrm{~s}, 3 \mathrm{H},-\mathrm{CH}_{3}\right)$; HRMS-ESI [M + Na] ${ }^{+}$Calcd for $\mathrm{C}_{14} \mathrm{H}_{20} \mathrm{O}_{4} \mathrm{Na}_{2} 275.1254$, Found 275.1248.

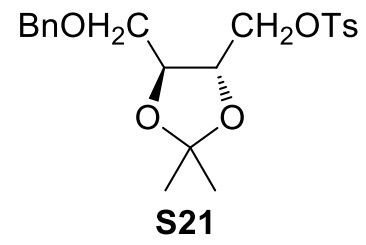

(4S,5S)-4-Benzyloxymethyl-5-tosyloxymethyl-2,2-dimethyl-1,3-dioxolan (S21) To a stirring solution of compound S20 (7.062 g, $0.03 \mathrm{~mol})$ in pyridine $(78 \mathrm{~mL})$ was added $p$-toluenesulfonyl chloride $(6.213 \mathrm{~g}, 0.04 \mathrm{~mol})$ at $0{ }^{\circ} \mathrm{C}$. The mixture was continuously stirred at this temperature for 4 h. The reaction mixture was extracted with ethyl acetate, washed with cold $1 \mathrm{~N} \mathrm{HCl}$, brine. The organic layer was dried over $\mathrm{MgSO}_{4}$ and concentrated under reduced pressure without further purification to give $11.264 \mathrm{~g}$ of a colorless liquid $\mathbf{S 2 1}$ in $99 \%$ yield: $R_{f}=0.40$ (ethyl acetate: hexanes = 1:4 (v/v)); $[\alpha]^{25}{ }_{\mathrm{D}}+64.2\left(\mathrm{c} 2.00, \mathrm{CHCl}_{3}\right)$; FT-IR (neat) $v_{\max } 3433,2988,2929,1604,1452,1365,1180,1094,979,821,669,549 \mathrm{~cm}^{-1} ;{ }^{1} \mathrm{H}$ NMR (400 MHz, $\left.\mathrm{CDCl}_{3}\right) \delta 7.77(\mathrm{~d}, J=8.4 \mathrm{~Hz}, 2 \mathrm{H}, \mathrm{ArH}), 7.37-7.29(\mathrm{~m}, 7 \mathrm{H}, \mathrm{ArH}), 4.54\left(\mathrm{~s}, 2 \mathrm{H},-\mathrm{OCH}_{2} \mathrm{Ar}\right), 4.22-3.99(\mathrm{~m}, 4 \mathrm{H}), 3.61 \mathrm{and} 3.54(\mathrm{ABX}$, $\left.J_{\mathrm{AB}}=10.2 \mathrm{~Hz}, J_{\mathrm{AX}}=4.8 \mathrm{~Hz}, J_{\mathrm{BX}}=4.8 \mathrm{~Hz}, 2 \mathrm{H},-\mathrm{CH}_{2} \mathrm{OBn}\right), 2.43\left(\mathrm{~s}, 3 \mathrm{H},-\mathrm{ArCH}_{3}\right), 1.37\left(\mathrm{~s}, 3 \mathrm{H},-\mathrm{CH}_{3}\right), 1.33\left(\mathrm{~s}, 3 \mathrm{H},-\mathrm{CH}_{3}\right)$; HRMS-ESI [M + Na] ${ }^{+}$ Calcd for $\mathrm{C}_{21} \mathrm{H}_{26} \mathrm{O}_{6} \mathrm{SNa} 429.1342$, Found 429.1376 .

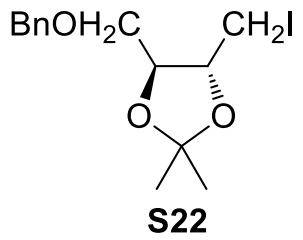

(4R,5S)-4-Benzyloxymethyl-5-iodomethyl-2,2-dimethyl-1,3-dioxolan (S22) To a stirring solution of compound S21 (0.965 g, $2.38 \mathrm{mmol})$ in acetonitrile $(7.9 \mathrm{~mL})$ was added sodium iodide $(0.534 \mathrm{~g}, 3.56 \mathrm{mmol})$ at $0{ }^{\circ} \mathrm{C}$. The mixture was heated at reflux for $3 \mathrm{~h}$. The reaction mixture was concentrated in vасио. The resulting yellowish syrup residue was purified by flash column chromatography on silica gel using ethyl acetate and hexanes $(1: 4, \mathrm{v} / \mathrm{v})$ as the eluent to get $0.791 \mathrm{~g}$ of $\mathbf{S 2 2}$ as a colorless liquid compound in $92 \%$ yield: $R_{f}=0.40$ (ethyl acetate:hexanes $=1: 4(\mathrm{v} / \mathrm{v})$ ); FT-IR (neat) $v_{\max } 3925,2988,2865,2634,1372,1244,1089,741 \mathrm{~cm}^{-1} ;[\alpha]^{27}{ }_{\mathrm{D}}+3.6\left(\mathrm{c} 0.40, \mathrm{CHCl}_{3}\right) ;{ }^{1} \mathrm{H} \mathrm{NMR}\left(400 \mathrm{MHz}, \mathrm{CDCl}_{3}\right) \delta 7.38-7.32(\mathrm{~m}$, $5 \mathrm{H}, \mathrm{ArH}), 4.59\left(\mathrm{~s}, 2 \mathrm{H},-\mathrm{OCH}_{2} \mathrm{Ar}\right), 3.99-3.95(\mathrm{~m}, 1 \mathrm{H}), 3.89-3.84(\mathrm{~m}, 1 \mathrm{H}), 3.65\left(\mathrm{dd}, J=5.0,3.2 \mathrm{~Hz}, 2 \mathrm{H},-\mathrm{CH}_{2} \mathrm{I}\right), 3.35 \mathrm{and} 3.28\left(\mathrm{ABX}, J_{\mathrm{AB}}=10.6 \mathrm{~Hz}\right.$, $\left.J_{\mathrm{AX}}=5.2 \mathrm{~Hz}, J_{\mathrm{BX}}=5.2 \mathrm{~Hz}, 2 \mathrm{H},-\mathrm{CH}_{2} \mathrm{OBn}\right), 1.47\left(\mathrm{~s}, 3 \mathrm{H},-\mathrm{CH}_{3}\right), 1.42\left(\mathrm{~s}, 3 \mathrm{H},-\mathrm{CH}_{3}\right) ; \mathrm{HRMS}-\mathrm{ESI}[\mathrm{M}+\mathrm{Na}]^{+} \mathrm{Calcd}$ for $\mathrm{C}_{21} \mathrm{H}_{26} \mathrm{O}_{6} \mathrm{SNa}_{385.9271}$, Found 429.9285 . 


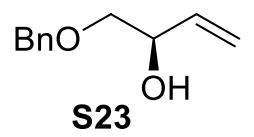

(R)-1-Benzyloxy-3-buten-2-ol (S23) To a stirring solution of compound S22 (0.020 g, $0.053 \mathrm{mmol})$ in THF (0.18 mL) was added Zn (0.028 g, $0.848 \mathrm{mmol})$ and $\mathrm{AcOH}(0.31 \mathrm{~mL}, 0.055 \mathrm{mmol})$ at $0{ }^{\circ} \mathrm{C}$. The mixture was continuously stirred at room temperature for $4 \mathrm{~h}$. The mixture was filtered through a short pad of Celite. The filtrate was extracted with ethyl acetate, washed with brine, dried over $\mathrm{MgSO}_{4}$, filtered, and concentrated in vасио. The observed yellowish syrup was purified by flash column chromatography on silica gel using ethyl acetate and hexanes (1:4, v/v) as the eluent to give $0.009 \mathrm{~g}$ of a colorless liquid $\mathbf{S 2 3}$ in $99 \%$ yield: $R_{f}=0.30$ (ethyl acetate: hexanes $=1: 4(\mathrm{v} / \mathrm{v})$ ); FT-IR (neat) $v_{\max } 3409,1635,1105$, $741 \mathrm{~cm}^{-1} ;[\alpha]^{26}{ }_{\mathrm{D}}+4.1\left(\mathrm{c} 1.00, \mathrm{CHCl}_{3}\right){ }^{1} \mathrm{H}$ NMR $\left(400 \mathrm{MHz}, \mathrm{CDCl}_{3}\right) \delta$ 7.39-7.29 (m, 5H, ArH), $4.58(\mathrm{~s}, 2 \mathrm{H},-\mathrm{OCH} \mathrm{Ar}), 5.84(\mathrm{ddd}, J=16.6,10.4$, $5.6 \mathrm{~Hz}, 1 \mathrm{H}, \mathrm{H}-3), 5.37$ (d, $J=16.6,1 \mathrm{H}), 5.20(\mathrm{~d}, J=10.4 \mathrm{~Hz}, 1 \mathrm{H}), 4.36(\mathrm{~m}, 1 \mathrm{H}), 3.55(\mathrm{dd}, J=9.6,3.6 \mathrm{~Hz}, 1 \mathrm{H}), 3.38(\mathrm{dd}, J=9.6,8.0 \mathrm{~Hz}, 1 \mathrm{H})$, 2.48 (bs, 1H, -OH); HRMS-ESI [M + Na $]^{+}$Calcd for $\mathrm{C}_{11} \mathrm{H}_{14} \mathrm{O}_{2} \mathrm{Na} 201.0886$, Found 201.0910.

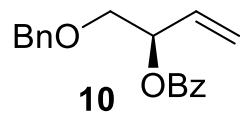

(R)-2-Benzoyloxy-1-benzyloxy-3-butene (10) To a stirring solution of compound $\mathbf{S 2 3}$ (3.335 g, $18.72 \mathrm{mmol})$ in pyridine (52 $\mathrm{mL})$ was added benzoyl chloride $(3.26 \mathrm{~mL}, 1.21 \mathrm{~g} / \mathrm{mL}, 28.08 \mathrm{mmol})$ at $0{ }^{\circ} \mathrm{C}$. The mixture was continuously stirred at this temperature for $4 \mathrm{~h}$. The reaction mixture was extracted with ethyl acetate, washed with cold $1 \mathrm{~N} \mathrm{HCl}$, brine. The organic layer was dried over $\mathrm{MgSO}_{4}$ and concentrated under reduced pressure without further purification to give $5.230 \mathrm{~g}$ of a colorless liquid 10 in $99 \%$ yield: $R_{f}=0.60$ (ethyl acetate:hexanes $=1: 4(\mathrm{v} / \mathrm{v})$ ); $[\alpha]^{25} \mathrm{D}$ +33.9 (c 0.53, $\mathrm{CHCl}_{3}$ ); FT-IR (neat) $v_{\max }$ 3066, 3031, 2861, 1964, 1721, 1648, 1594, 1490, 1453, 1417, 1358, 1208, 1170, 1106, 1028, 992, 935, 858, 807, 707, 608, $468 \mathrm{~cm}^{-1}$; ${ }^{1} \mathrm{H} \mathrm{NMR}\left(400 \mathrm{MHz}, \mathrm{CDCl}_{3}\right) \delta 8.09(\mathrm{~d}, J=8.0 \mathrm{~Hz}, 2 \mathrm{H}, \mathrm{ArH}), 7.57(\mathrm{t}, J=7.4 \mathrm{~Hz}, 1 \mathrm{H}, \mathrm{ArH}), 7.45(\mathrm{t}, J=7.6 \mathrm{~Hz}, 2 \mathrm{H}$, ArH), 7.33-7.27 (m, 5H, ArH), 5.92 (ddd, $J=17.2,10.5,5.6 \mathrm{~Hz}, 1 \mathrm{H}, \mathrm{H}-3), 5.77-5.73$ (m, 1H, H-2), 5.42 (d, $J=17.2 \mathrm{~Hz}, 1 \mathrm{H}, \mathrm{H}-4 \mathrm{a}), 5.29$ (d, $J=$ $10.5 \mathrm{~Hz}, 1 \mathrm{H}, \mathrm{H}-4 \mathrm{~b}), 4.64$ and $4.58\left(\mathrm{ABq}, J=12.2 \mathrm{~Hz}, 2 \mathrm{H},-\mathrm{OCH}_{2} \mathrm{Ar}\right), 3.74$ and $3.70\left(\mathrm{ABX}, J_{\mathrm{AB}}=10.4 \mathrm{~Hz}, J_{\mathrm{AX}}=6.4 \mathrm{~Hz}, J_{\mathrm{BX}}=4.4 \mathrm{~Hz}, 2 \mathrm{H}, \mathrm{H}-1\right)$; ${ }^{13} \mathrm{C}$ NMR $\left(100 \mathrm{MHz}, \mathrm{CDCl}_{3}\right) \delta 165.8(\mathrm{C}), 138.0(\mathrm{C}), 133.5(\mathrm{CH}), 133.1(\mathrm{CH}), 130.3(\mathrm{C}), 129.8(\mathrm{CH}), 128.5(\mathrm{CH}), 128.5(\mathrm{CH}), 127.8(\mathrm{CH}), 127.7$ $(\mathrm{CH}), 118.1\left(\mathrm{CH}_{2}\right), 77.6(\mathrm{C}), 77.3(\mathrm{C}), 76.9(\mathrm{C}), 73.8(\mathrm{CH}), 73.2\left(\mathrm{CH}_{2}\right), 71.4\left(\mathrm{CH}_{2}\right)$; HRMS-ESI $[\mathrm{M}+\mathrm{Na}]^{+} \mathrm{Calcd}$ for $\mathrm{C}_{18} \mathrm{H}_{18} \mathrm{O}_{3} \mathrm{Na} 305.1148$, Found 305.1159 .

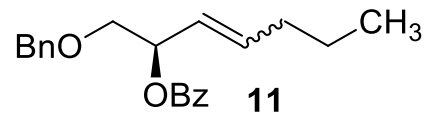

(2R,3E)-2-(Benzoyloxy)-1-(benzyloxy)-3-tetracosene (11E) and (2R,3Z)-2- (benzoyloxy)-1-(benzyloxy)-3-tetracosene (11Z) To a solution of the alkene $10(0.605 \mathrm{~g}, 2.13 \mathrm{mmol})$ and 1-docosene $(1.315 \mathrm{~g}, 4.26 \mathrm{mmol})$ in anhydrous $\mathrm{CH}_{2} \mathrm{Cl}_{2}(71 \mathrm{~mL})$ was added Grubbs II catalyst (0.018 g, $21.28 \mu \mathrm{mol})$ and then the mixture was heated at reflux for $24 \mathrm{~h}$. The reaction mixture was concentrated in vacuo and the resulting yellowish syrup residue was purified by flash column chromatography on silica gel using $\mathrm{CH}_{2} \mathrm{Cl}_{2}$ and hexanes $(1: 2, \mathrm{v} / \mathrm{v})$ as the eluent to get $1.198 \mathrm{~g}$ of a colorless liquid 11 as a ca. 9.8:1 mixture of $E$ - and $Z$-stereoisomers in 99\% yield: $R_{f}=0.25\left(\mathrm{CH}_{2} \mathrm{Cl}_{2}:\right.$ hexanes = 1:2 $\left.(\mathrm{v} / \mathrm{v})\right) ;[\alpha]^{23}{ }_{\mathrm{D}}+9.6(\mathrm{c} 0.57, \mathrm{CHCl}) ; \mathrm{FT}$ IR (neat) $v_{\max } 2923,2853,1722,1516,1459,1267,1106,1026,968,707 \mathrm{~cm}^{-1} ;{ }^{1} \mathrm{H}$ NMR $\left(400 \mathrm{MHz}, \mathrm{CDCl}_{3}\right) \delta 8.08-8.06$ (m, 2H, ArH), 7.58-7.54 (m, 1H, ArH), 7.46-7.42 (m, 2H, ArH), 7.34-7.27 (m, 5H, ArH), 5.88 (dt, J=15.3, 7.0 Hz, 1H, H-4), 5.71 (td, J = 6.8, 4.2 Hz, 1H, H-2), 5.58-5.52 $(\mathrm{m}, 1 \mathrm{H}, \mathrm{H}-3), 4.63,4.57(\mathrm{ABq}, J=12.2 \mathrm{~Hz}, 2 \mathrm{H}, \mathrm{CHaHbPh}), 3.71,3.66\left(\mathrm{ABX}, J_{\mathrm{AB}}=10.7 \mathrm{~Hz}, J_{\mathrm{AX}}=6.8 \mathrm{~Hz}, J_{\mathrm{BX}}=4.2 \mathrm{~Hz}, 2 \mathrm{H}, \mathrm{H}-1 \mathrm{a}, \mathrm{H}-1 \mathrm{~b}\right), 2.04(\mathrm{q}$, $J=7.0 \mathrm{~Hz}, 2 \mathrm{H}, \mathrm{H}-5), 1.40-1.24\left(\mathrm{~m}, 36 \mathrm{H}\right.$, aliphatic), $0.88(\mathrm{t}, J=6.8 \mathrm{~Hz}, 3 \mathrm{H}) ;{ }^{13} \mathrm{C} \mathrm{NMR}\left(100 \mathrm{MHz}, \mathrm{CDCl}_{3}\right) \delta 165.7(\mathrm{C}), 138.1(\mathrm{C}), 136.0(\mathrm{CH})$, $132.8(\mathrm{CH}), 130.6(\mathrm{C}), 129.7(\mathrm{CH}), 128.3(\mathrm{CH}), 128.3(\mathrm{CH}), 127.6(\mathrm{CH}), 127.6(\mathrm{CH}), 125.0(\mathrm{CH}), 73.9(\mathrm{CH}), 73.1\left(\mathrm{CH}_{2}\right), 71.8\left(\mathrm{CH}_{2}\right), 32.4\left(\mathrm{CH}_{2}\right)$, $32.0\left(\mathrm{CH}_{2}\right), 29.8\left(\mathrm{CH}_{2}\right), 29.8\left(\mathrm{CH}_{2}\right), 29.8\left(\mathrm{CH}_{2}\right), 29.7\left(\mathrm{CH}_{2}\right), 29.5\left(\mathrm{CH}_{2}\right), 29.5\left(\mathrm{CH}_{2}\right), 29.2\left(\mathrm{CH}_{2}\right), 28.9\left(\mathrm{CH}_{2}\right), 22.8\left(\mathrm{CH}_{2}\right), 14.2\left(\mathrm{CH}_{3}\right) ; \mathrm{HRMS}-\mathrm{ESI}$ $[\mathrm{M}+\mathrm{Na}]^{+}$Calcd for $\mathrm{C}_{38} \mathrm{H}_{58} \mathrm{O}_{3} \mathrm{Na} 585.4278$, Found 585.4288.

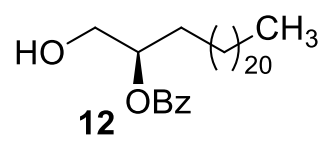

(R)-2-(Benzyloxy)-tetracosan-1-ol (12) $20 \% \mathrm{Pd}(\mathrm{OH})_{2} / \mathrm{C}(0.121 \mathrm{~g})$ was added to a solution of alkene 11 (1.702 $\left.\mathrm{g}, 3.02 \mathrm{mmol}\right)$ in $\mathrm{MeOH} / \mathrm{ethyl}$ acetate $(30 \mathrm{~mL}, 1 / 1(\mathrm{v} / \mathrm{v}))$. The reaction mixture was stirred under hydrogen $(50 \mathrm{psi})$ at room temperature for $3 \mathrm{~h}$. The $\mathrm{Pd}(\mathrm{OH})_{2} / \mathrm{C}$ was removed through a short pad of $\mathrm{SiO} 2 / \mathrm{Ce}$ lite and the filter was washed with ethyl acetate. The filtrate was concentrated in vacuo to obtain $1.420 \mathrm{~g}$ of $\mathbf{1 2}$ as a 
white solid in $99 \%$ yield: $R_{f}=0.43$ (ethyl acetate: hexanes $=1: 3(\mathrm{v} / \mathrm{v})$ ); $[\alpha]^{23}{ }_{\mathrm{D}}+20.5\left(\mathrm{c} 0.32, \mathrm{CHCl}_{3}\right)$; FT-IR (neat) $v_{\max } 3437,2919,2851,1707$, 1644, 1549, 1462, 1276, 1117, 1063, 758, $714 \mathrm{~cm}^{-1}$; ${ }^{1} \mathrm{H}$ NMR (400 MHz, $\mathrm{CDCl}_{3}$ ) 8 8.07-8.05 (m, 2H, ArH), 7.59-7.56 (m, 1H, ArH), 7.47-7.43 (m, $2 \mathrm{H}, \mathrm{ArH}), 5.19-5.14(\mathrm{~m}, 1 \mathrm{H}, \mathrm{H}-2), 3.84,3.77\left(\mathrm{ABX}, J_{\mathrm{AB}}=12.1 \mathrm{~Hz}, J_{\mathrm{AX}}=3.3 \mathrm{~Hz}, J_{\mathrm{BX}}=6.1 \mathrm{~Hz}, 2 \mathrm{H}, \mathrm{H}-1 \mathrm{a}, \mathrm{H}-1 \mathrm{~b}\right), 1.81-1.65(\mathrm{~m}, 2 \mathrm{H}, \mathrm{H}-3), 1.43-$ 1.25 (m, 40H, aliphatic), $0.88\left(\mathrm{t}, J=6.7 \mathrm{~Hz}, 3 \mathrm{H}, \mathrm{CH}_{3}\right.$ ); HRMS-ESI $[\mathrm{M}+\mathrm{Na}]^{+}$Calcd for $\mathrm{C}_{31} \mathrm{H}_{54} \mathrm{O}_{3} \mathrm{Na}$ 497.3965, Found 497.3972.

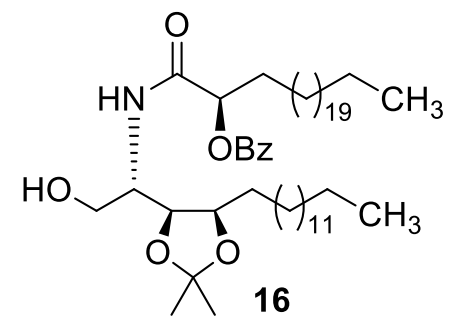

(2S,3S,4R)-2-[(2R)-2-(Benzoyloxy)tetracosanoylamino]-3,4-O-isopropylideneoctadecan-1,3,4-triol (16) To a stirring solution of compound 12 $(0.407 \mathrm{~g}, 0.86 \mathrm{mmol})$ and IBX $(0.472 \mathrm{~g}, 1.72 \mathrm{mmol})$ in $\mathrm{ACN}(8.4 \mathrm{~mL})$ at reflux for $4 \mathrm{~h}$. The mixture was filtered through a short pad of Celite. The filtrate was concentrated under reduced pressure to give a colorless syrup aldehye $\left(R_{f}=0.63\right.$ (ethyl acetate: hexanes $\left.=1: 3(\mathrm{v} / \mathrm{v})\right)$. The aldehye, without further purification, was dissolved in tert-butanol/ $\mathrm{H}_{2} \mathrm{O}(8.4 \mathrm{~mL}, 10 / 1(\mathrm{v} / \mathrm{v}))$ and then $\mathrm{NaClO}_{2}(0.227 \mathrm{~g}, 2.58 \mathrm{mmol})$ and $\mathrm{NaH}_{2} \mathrm{PO}_{4}(0.116 \mathrm{~g}$, $0.86 \mathrm{mmol}$ ) was added to this solution at $0{ }^{\circ} \mathrm{C}$. The mixture was warmed to room temperature and continuously stirred at this temperature for $4 \mathrm{~h}$. The reaction mixture was extracted with ethyl acetate, washed with brine, dried over $\mathrm{MgSO}_{4}$, and concentrated in vacuo. The resulting white solid residue $\left(R_{f}=0.15\right.$ (ethyl acetate: hexanes $=1: 2(\mathrm{v} / \mathrm{v})$ ), without further purification, was dissolved in $\mathrm{CH}_{2} \mathrm{Cl}_{2}(8.4 \mathrm{~mL})$ and then EDC $(0.243 \mathrm{~g}, 1.29$ mmol), DMAP $(0.010 \mathrm{~g}, 0.09 \mathrm{mmol})$ and $N$-hydroxysuccinimide $(0.116 \mathrm{~g}, 1.03 \mathrm{mmol})$ was added to this solution at $0{ }^{\circ} \mathrm{C}$. The mixture was warmed to room temperature and continuously stirred at this temperature for $2 \mathrm{~h}$. The reaction mixture was extracted with ethyl acetate, washed with brine, dried over $\mathrm{MgSO}_{4}$, and concentrated in vacuo. The resulting yellowish solid residue $\left(R_{f}=0.48\right.$ (ethyl acetate: hexanes $=1: 2(\mathrm{v} / \mathrm{v})$ ), without further purification, was dissolved in THF $(17 \mathrm{~mL})$ and then $15(0.332 \mathrm{~g}, 0.95 \mathrm{mmol})$ and $\mathrm{Et}_{3} \mathrm{~N}(0.27 \mathrm{~mL}, 1.98 \mathrm{mmol})$ was added to this solution at $0{ }^{\circ} \mathrm{C}$. The mixture was warmed to $50{ }^{\circ} \mathrm{C}$ and continuously stirred at this temperature for $4 \mathrm{~h}$. The reaction mixture was concentrated in vacuo and the resulting yellowish syrup residue was purified by flash column chromatography on silica gel using ethyl acetate and hexanes $(1: 2$, v/v) as the eluent to get $0.592 \mathrm{~g}$ of a white solid $\mathbf{1 6}$ in $83 \%$ yield: $R_{f}=0.10$ (ethyl acetate: hexanes $=1: 3(\mathrm{v} / \mathrm{v})$ ); $\mathrm{mp}=83-84{ }^{\circ} \mathrm{C} ;[\alpha]^{23}{ }_{\mathrm{D}}+10.8\left(\mathrm{c} 0.52, \mathrm{CHCl}_{3}\right)$; FT-IR (neat) $v_{\max } 3437,3288,3097,2921,2853,1729,1656,1557,1462,1375,1263,1171,1109,1059,869,765,710 \mathrm{~cm}^{-1} ;{ }^{1} \mathrm{H} \mathrm{NMR}(400 \mathrm{MHz}$, $\left.\mathrm{CDCl}_{3}\right) \delta 8.07(\mathrm{~d}, J=7.5 \mathrm{~Hz}, 2 \mathrm{H}, \mathrm{ArH}), 7.61(\mathrm{t}, J=7.5 \mathrm{~Hz}, 1 \mathrm{H}, \mathrm{ArH}), 7.48(\mathrm{t}, J=7.5 \mathrm{~Hz}, 2 \mathrm{H}, \mathrm{ArH}), 6.59(\mathrm{~d}, J=8.5 \mathrm{~Hz}, 1 \mathrm{H}, \mathrm{NH}), 5.36(\mathrm{t}, J=6.0$ Hz, 1H, H-2'), 4.19-4.14 (m, 2H, H-4, H-3), 4.11-4.09 (m, 1H, H-2), 3.87 (d, J = 10.8 Hz, 1H, H-1a), 3.68-3.63 (m, 1H, H-1b), 2.43 (bs, 1H, OH1), 2.00-1.95 (m, 2H, H-3'), 1.46 (s, 3H, $\left.\left(\mathrm{CH}_{3}\right)_{2} \mathrm{C}\right), 1.34$ (s, 3H, $\left.\left(\mathrm{CH}_{3}\right)_{2} \mathrm{C}\right), 1.25$ (s, 66H, aliphatic), $0.88\left(\mathrm{t}, J=6.6 \mathrm{~Hz}, 6 \mathrm{H}, \mathrm{CH}_{3}\right) ;{ }^{13} \mathrm{C} \mathrm{NMR}(100$ $\left.\mathrm{MHz}, \mathrm{CDCl}_{3}\right) \delta 169.8(\mathrm{C}), 165.6(\mathrm{C}), 133.4(\mathrm{CH}), 129.7(\mathrm{CH}), 129.3(\mathrm{C}), 128.4(\mathrm{CH}), 108.0(\mathrm{C}), 77.8(\mathrm{CH}), 76.6(\mathrm{CH}), 74.8(\mathrm{CH}), 62.6(\mathrm{CH})$, $50.3(\mathrm{CH}), 31.9\left(\mathrm{CH}_{2}\right), 31.8\left(\mathrm{CH}_{2}\right), 29.6\left(\mathrm{CH}_{2}\right), 29.5\left(\mathrm{CH}_{2}\right), 29.4\left(\mathrm{CH}_{2}\right), 29.3\left(\mathrm{CH}_{2}\right), 27.8\left(\mathrm{CH}_{3}\right), 26.7\left(\mathrm{CH}_{2}\right), 25.4\left(\mathrm{CH}_{3}\right), 25.0\left(\mathrm{CH}_{2}\right), 22.7\left(\mathrm{CH}_{2}\right)$, $14.1\left(\mathrm{CH}_{3}\right)$; HRMS-ESI $[\mathrm{M}+\mathrm{Na}]^{+}$Calcd for $\mathrm{C}_{52} \mathrm{H}_{93} \mathrm{NO}_{6} \mathrm{Na} 850.6895$, Found 850.6893 . 
(G) Synthesis of the amino-disaccharide 3:
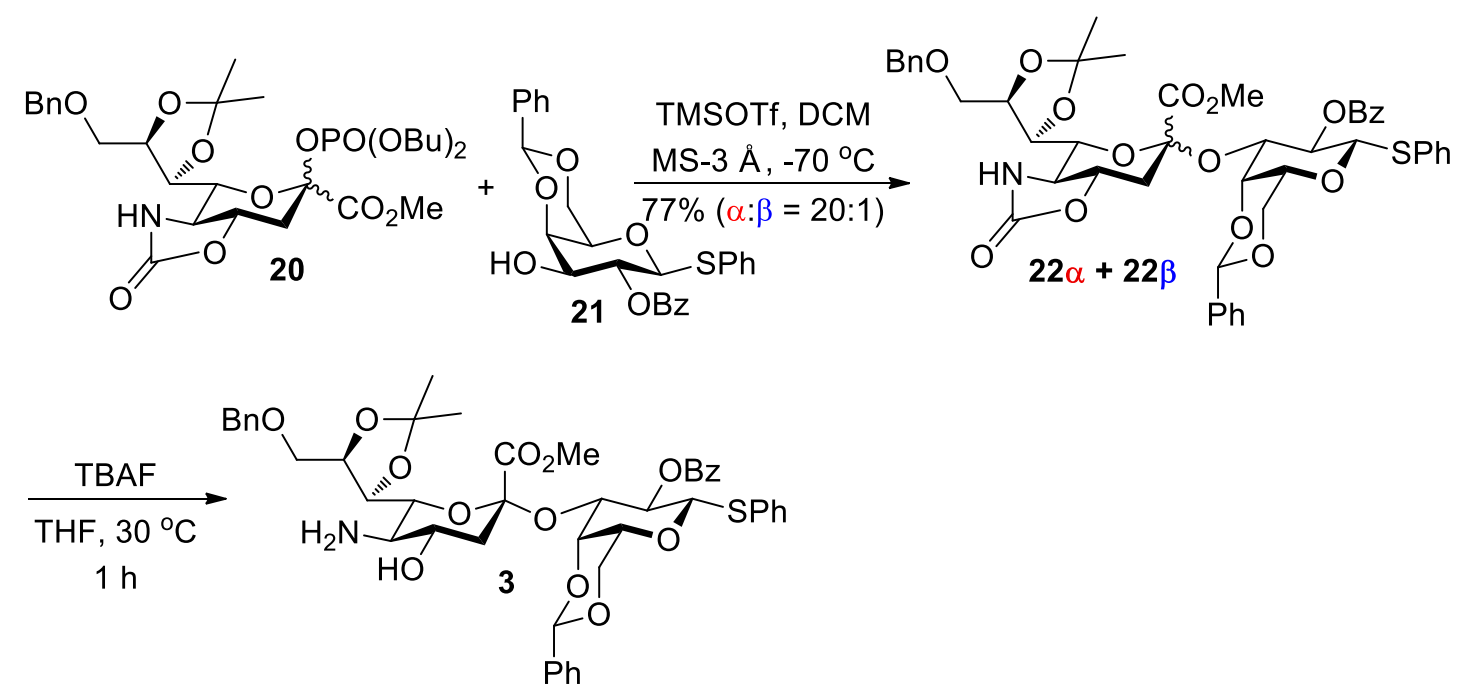

Scheme S6. Synthesis of the amino-disaccharide 3 .

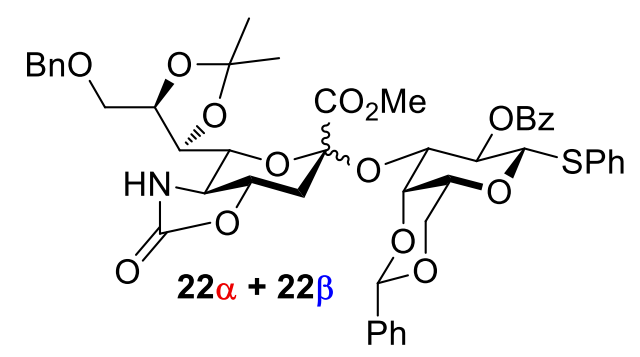

Methyl 5-amino-9-O-benzyl-5-N,4-O-carbonyl-3,5-dideoxy-7,8-O-isopropylidene-D-glycero- $\alpha$-D-galacto-2-nonulopyranosylonate-(2 $\rightarrow$ 3)phenyl 2-O-benzoyl-4,6-O-benzylidene-1-thio- $\beta$-D-galactopyranoside $(22 \alpha)$ and methyl 5-amino-9-O-benzyl-5- $\mathrm{N}, 4-\mathrm{O}$-carbonyl-3,5-dideoxy7,8- $O$-isopropylidene-D-glycero- $\beta$-D-galacto-2-nonulopyranosylonate- $(2 \quad \rightarrow \quad 3)$-phenyl 2-O-benzoyl-4,6- $O$-benzylidene-1-thio- $\beta$-Dgalactopyranoside (22ß) A mixture of the sialyl donor 20 (0.501 g, 0.80 mmol), acceptor 21 (0.308 g, 0.66 mmol), and activated $3 \AA$ powdered molecular sieves $(0.883 \mathrm{~g})$ in anhydrous $\mathrm{CH}_{2} \mathrm{Cl}_{2}(6.6 \mathrm{~mL})$ was stirred at room temperature for $0.5 \mathrm{~h}$ under nitrogen to remove any trace amounts of water. The reaction mixture was then cooled to $-70{ }^{\circ} \mathrm{C}$ followed by addition of TMSOTf $(0.25 \mathrm{~mL}, 1.33 \mathrm{mmol})$. Continuously stirred at this temperature for $1.5 \mathrm{~h}$, the reaction mixture was carefully quenched with triethylamine and then filtered through a short pad of Celite. The filtrate was washed with cold saturated aqueous $\mathrm{NaHCO}_{3}$, brine, dried over $\mathrm{MgSO}_{4}$, filtered, and concentrated in vacuo. The observed yellowish syrup was purified by flash column chromatography on silica gel using ethyl acetate and hexanes (1:1, v/v) as the eluent to give $0.429 \mathrm{~g}$ of a white solid $\mathbf{2 2} \boldsymbol{\alpha}$ in $73 \%$ yield, $0.023 \mathrm{~g}$ of a white solid $\mathbf{2 2} \boldsymbol{\beta}$ in $4 \%$ yield: $\mathbf{2 2 \alpha}: R_{f}=0.23$ (ethyl acetate: hexanes $=1: 1(\mathrm{v} / \mathrm{v})$ ); $\mathrm{mp}=132-133{ }^{\circ} \mathrm{C} ;[\alpha]^{23} \mathrm{D}-29.8(\mathrm{c}$ $0.21, \mathrm{CHCl}_{3}$ ); FT-IR (neat) $v_{\max } 3443,3314,3071,2922,2854,1728,1664,1550,1455,1367,1314,1265,1173,1082,867,702 \mathrm{~cm}^{-1}$; ${ }^{1} \mathrm{H} \mathrm{NMR}$ $\left(400 \mathrm{MHz}, \mathrm{CDCl}_{3}\right) \delta 8.01(\mathrm{~d}, J=7.3 \mathrm{~Hz}, 2 \mathrm{H}, \mathrm{ArH}), 7.60(\mathrm{t}, J=7.3 \mathrm{~Hz}, 1 \mathrm{H}, \mathrm{ArH}), 7.56(\mathrm{~d}, J=7.1 \mathrm{~Hz}, 2 \mathrm{H}, \mathrm{ArH}), 7.47(\mathrm{t}, J=7.7 \mathrm{~Hz}, 2 \mathrm{H}, \mathrm{ArH})$, 7.45-7.42 (m, 2H, ArH), 7.37-7.28 (m, 9H, ArH), 7.25-7.21 (m, 2H, ArH), 5.49 (t, J = 9.8 Hz, 1H, H-2 $\left.{ }^{\mathrm{Gal}}\right), 5.41(\mathrm{~s}, 1 \mathrm{H}, \mathrm{CHPh}), 4.95$ (s, 1H, NH), $4.76\left(\mathrm{~d}, J=9.8 \mathrm{~Hz}, 1 \mathrm{H}, \mathrm{H}-1^{\mathrm{Gal}}\right), 4.59(\mathrm{~s}, 2 \mathrm{H}, \mathrm{CH} / \mathrm{Ph}), 4.56-4.51\left(\mathrm{~m}, 1 \mathrm{H}, \mathrm{H}-8^{\mathrm{Neu}}\right), 4.49\left(\mathrm{dd}, J=9.8,3.4 \mathrm{~Hz}, 1 \mathrm{H}, \mathrm{H}-3^{\mathrm{Gal}}\right), 4.43(\mathrm{~d}, J=3.4 \mathrm{~Hz}, 1 \mathrm{H}, \mathrm{H}-$ $\left.4^{\mathrm{Gal}}\right), 4.32\left(\mathrm{~d}, J=12.3 \mathrm{~Hz}, 1 \mathrm{H}, \mathrm{H}-6 \mathrm{a}^{\mathrm{Gal}}\right), 4.10,3.98\left(\mathrm{ABX}, J_{\mathrm{AB}}=10.4 \mathrm{~Hz}, J_{\mathrm{AX}}=6.6 \mathrm{~Hz}, J_{\mathrm{BX}}=5.4 \mathrm{~Hz}, 2 \mathrm{H}, \mathrm{H}-9 \mathrm{a}^{\mathrm{Neu}}, \mathrm{H}-9 \mathrm{~b}^{\mathrm{Neu}}\right), 4.08(\mathrm{dd}, J=6.7,1.6$ $\left.\mathrm{Hz}, 1 \mathrm{H}, \mathrm{H}-7^{\mathrm{Neu}}\right), 3.89\left(\mathrm{dd}, J=12.5,0.9 \mathrm{~Hz}, 1 \mathrm{H}, \mathrm{H}-6 \mathrm{~b}^{\mathrm{Gal}}\right), 3.86\left(\mathrm{dd}, J=12.5,1.6 \mathrm{~Hz}, 1 \mathrm{H}, \mathrm{H}-6^{\mathrm{Neu}}\right), 3.80-3.73\left(\mathrm{~m}, 1 \mathrm{H}, \mathrm{H}-4^{\mathrm{Neu}}\right), 3.49(\mathrm{t}, J=10.6 \mathrm{~Hz}$, $\left.1 \mathrm{H}, \mathrm{H}-5^{\mathrm{Neu}}\right), 3.43-3.42\left(\mathrm{~m}, 1 \mathrm{H}, \mathrm{H}-5^{\mathrm{Gal}}\right), 3.42\left(\mathrm{~s}, 3 \mathrm{H}, \mathrm{CO}_{2} \mathrm{CH}_{3}\right), 2.78\left(\mathrm{dd}, J=12.3,3.1 \mathrm{~Hz}, 1 \mathrm{H}, \mathrm{H}-3 \mathrm{eq}{ }^{\mathrm{Neu}}\right), 1.79\left(\mathrm{t}, J=12.3 \mathrm{~Hz}, 1 \mathrm{H}, \mathrm{H}-3 \mathrm{ax}{ }^{\mathrm{Neu}}\right), 1.56$ $\left(\mathrm{s}, 3 \mathrm{H},\left(\mathrm{CH}_{3}\right)_{2} \mathrm{C}\right), 1.40\left(\mathrm{~s}, 3 \mathrm{H},\left(\mathrm{CH}_{3}\right)_{2} \mathrm{C}\right){ }^{13} \mathrm{C}$ NMR (100 MHz, $\left.\mathrm{CDCl}_{3}\right) \delta 168.4(\mathrm{C}), 164.7(\mathrm{C}), 160.0(\mathrm{C}), 137.7(\mathrm{C}), 137.5(\mathrm{C}), 133.4(\mathrm{CH}), 133.2$ $(\mathrm{CH}), 131.5(\mathrm{C}), 129.7(\mathrm{C}), 129.6(\mathrm{CH}), 129.0(\mathrm{CH}), 128.7(\mathrm{CH}), 128.4(\mathrm{CH}), 128.4(\mathrm{CH}), 128.1(\mathrm{CH}), 128.0(\mathrm{CH}), 127.8(\mathrm{CH}), 127.8(\mathrm{CH}), 126.4$ $(\mathrm{CH}), 108.9(\mathrm{C}), 100.8(\mathrm{CH}), 99.4(\mathrm{C}), 85.5(\mathrm{CH}), 76.8(\mathrm{CH}), 76.0(\mathrm{CH}), 75.1(\mathrm{CH}), 74.8(\mathrm{CH}), 73.8(\mathrm{CH}), 73.8(\mathrm{CH}), 73.3(\mathrm{CH}), 69.5(\mathrm{CH}), 69.1$ $\left(\mathrm{CH}_{2}\right), 68.5\left(\mathrm{CH}_{2}\right), 67.7(\mathrm{CH}), 57.6(\mathrm{CH}), 52.9\left(\mathrm{CH}_{3}\right), 36.7\left(\mathrm{CH}_{2}\right), 26.8\left(\mathrm{CH}_{3}\right), 24.7\left(\mathrm{CH}_{3}\right)$; HRMS-ESI [M + Na] ${ }^{+} \mathrm{Calcd}$ for $\mathrm{C}_{47} \mathrm{H}_{49} \mathrm{NO}{ }_{14} \mathrm{SNa}$

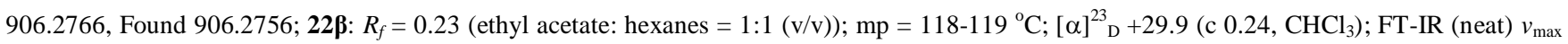
3519, 3280, 2995, 2869, 1763, 1594, 1447, 1374, 1262, 1092, 1015, 945, 863, 807, 752, $704 \mathrm{~cm}^{-1} ;{ }^{1} \mathrm{H} \mathrm{NMR}(400 \mathrm{MHz}, \mathrm{CDCl}) \delta 8.04(\mathrm{~d}, J=7.8$ $\mathrm{Hz}, 2 \mathrm{H}, \mathrm{ArH}), 7.60(\mathrm{t}, J=7.2 \mathrm{~Hz}, 1 \mathrm{H}, 2 \mathrm{H}, \mathrm{ArH}), 7.54(\mathrm{~d}, J=7.8 \mathrm{~Hz}, 2 \mathrm{H}, 2 \mathrm{H}, \mathrm{ArH}), 7.48(\mathrm{t}, J=7.8 \mathrm{~Hz}, 2 \mathrm{H}, 2 \mathrm{H}, \mathrm{ArH}), 7.46-7.39(\mathrm{~m}, 5 \mathrm{H}, 2 \mathrm{H}, \mathrm{ArH})$, 
7.32-7.27 (m, 4H, 2H, ArH), 7.20-7.15 (m, 4H, 2H, ArH), $5.64(\mathrm{~s}, 1 \mathrm{H}, \mathrm{CHPh}), 5.49$ (s, 1H, NH), $5.42\left(\mathrm{t}, J=9.8 \mathrm{~Hz}, 1 \mathrm{H}, \mathrm{H}-2^{\mathrm{Gal}}\right), 4.84(\mathrm{~d}, J=9.8$ $\left.\mathrm{Hz}, 1 \mathrm{H}, \mathrm{H}-1^{\mathrm{Gal}}\right), 4.55\left(\mathrm{~d}, J=3.2 \mathrm{~Hz}, 1 \mathrm{H}, \mathrm{H}-4^{\mathrm{Gal}}\right), 4.47-4.40\left(\mathrm{~m}, 1 \mathrm{H}, \mathrm{H}-4^{\mathrm{Neu}}\right), 4.38\left(\mathrm{~d}, J=11.8 \mathrm{~Hz}, 1 \mathrm{H}, \mathrm{H}-6 \mathrm{a}^{\mathrm{Gal}}\right), 4.25(\mathrm{dd}, J=10.0,7.2 \mathrm{~Hz}, 1 \mathrm{H}, \mathrm{H}-$ $\left.6^{\mathrm{Neu}}\right), 4.20-4.13\left(\mathrm{~m}, 2 \mathrm{H}, \mathrm{H}-8^{\mathrm{Neu}}, \mathrm{H}-7^{\mathrm{Neu}}\right), 4.08,4.02(\mathrm{ABq}, J=11.7 \mathrm{~Hz}, 2 \mathrm{H}, \mathrm{CHaHbPh}), 4.01-3.96\left(\mathrm{~m}, 2 \mathrm{H}, \mathrm{H}-6 \mathrm{~b}^{\mathrm{Gal}}, \mathrm{H}-3^{\mathrm{Gal}}\right), 3.54$ (s, $\left.1 \mathrm{H}, \mathrm{H}-5^{\mathrm{Gal}}\right)$, $3.37\left(\mathrm{~s}, 3 \mathrm{H}, \mathrm{CO}_{2} \mathrm{CH}_{3}\right), 3.36,3.29\left(\mathrm{ABX}, J_{\mathrm{AB}}=10.3 \mathrm{~Hz}, J_{\mathrm{AX}}=5.3 \mathrm{~Hz}, J_{\mathrm{BX}}=4.3 \mathrm{~Hz}, 2 \mathrm{H}, \mathrm{H}-9 \mathrm{a}^{\mathrm{Neu}}, \mathrm{H}-9 \mathrm{~b}^{\mathrm{Neu}}\right), 3.21\left(\mathrm{t}, J=10.0 \mathrm{~Hz}, 1 \mathrm{H}, \mathrm{H}-5^{\mathrm{Neu}}\right), 2.51$ $\left(\mathrm{dd}, J=12.2,3.8 \mathrm{~Hz}, 1 \mathrm{H}, \mathrm{H}-3 \mathrm{eq}^{\mathrm{Neu}}\right), 1.80$ (t, $\left.J=12.2 \mathrm{~Hz}, 1 \mathrm{H}, \mathrm{H}-3 \mathrm{ax}^{\mathrm{Neu}}\right), 1.49$ (s, $\left.3 \mathrm{H},\left(\mathrm{CH}_{3}\right)_{2} \mathrm{C}\right), 1.35\left(\mathrm{~s}, 3 \mathrm{H},\left(\mathrm{CH}_{3}\right)_{2} \mathrm{C}\right) ;{ }^{13} \mathrm{C} \mathrm{NMR}(100 \mathrm{MHz}$, $\left.\mathrm{CDCl}_{3}\right) \delta 166.7(\mathrm{C}), 164.9(\mathrm{C}), 158.9(\mathrm{C}), 137.5(\mathrm{C}), 137.0(\mathrm{C}), 133.9(\mathrm{CH}), 133.2(\mathrm{CH}), 131.0(\mathrm{C}), 130.3(\mathrm{C}), 129.8(\mathrm{CH}), 129.4(\mathrm{CH}), 128.9$ $(\mathrm{CH}), 128.6(\mathrm{CH}), 128.6(\mathrm{CH}), 128.5(\mathrm{CH}), 128.4(\mathrm{CH}), 128.2(\mathrm{CH}), 128.1(\mathrm{CH}), 126.2(\mathrm{CH}), 109.1(\mathrm{C}), 100.5(\mathrm{C}), 100.3(\mathrm{CH}), 85.1(\mathrm{CH}), 77.7$ $(\mathrm{CH}), 77.0(\mathrm{CH}), 76.6(\mathrm{CH}), 76.6(\mathrm{CH}), 74.6(\mathrm{CH}), 73.7(\mathrm{CH}), 73.3\left(\mathrm{CH}_{2}\right), 69.7\left(\mathrm{CH}_{2}\right), 69.7(\mathrm{CH}), 69.3\left(\mathrm{CH}_{2}\right), 67.8(\mathrm{CH}), 58.6(\mathrm{CH}), 52.9(\mathrm{CH})$, $37.8\left(\mathrm{CH}_{2}\right), 28.0\left(\mathrm{CH}_{3}\right), 25.6\left(\mathrm{CH}_{3}\right)$; HRMS-ESI $[\mathrm{M}+\mathrm{Na}]^{+}$Calcd for $\mathrm{C}_{47} \mathrm{H}_{49} \mathrm{NO}_{14} \mathrm{SNa} 906.2766$, Found 906.2758.

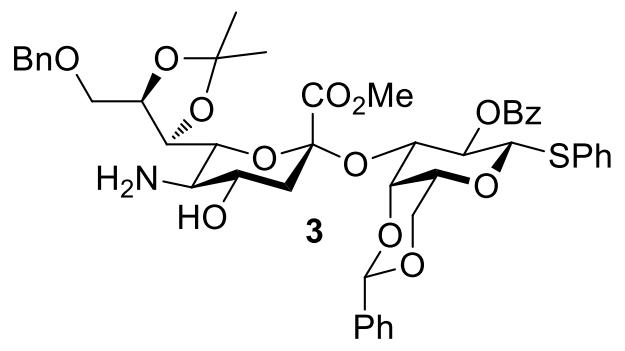

Methyl 5-amino-9-O-benzyl-3,5-dideoxy-7,8- $O$-isopropylidene-D-glycero- $\alpha$-D-galacto-2-nonulopyranosylonate-(2 $\rightarrow 3$ )-phenyl 2- $O$-benzoyl4,6- $\boldsymbol{O}$ - benzylidene-1-thio- $\boldsymbol{\beta}$-D-galactopyranoside (3) To a stirring solution of compound $\mathbf{2 2} \boldsymbol{\alpha}(0.160 \mathrm{~g}, 0.18 \mathrm{mmol})$ in $\mathrm{THF}(6.0 \mathrm{~mL})$ was added tetra- $n$-butylammonium fluoride $(0.90 \mathrm{~mL}, 0.90 \mathrm{mmol})$ at $0{ }^{\circ} \mathrm{C}$. The mixture was warmed to $30{ }^{\circ} \mathrm{C}$ and continuously stirred at this temperature for $1 \mathrm{~h}$. The reaction mixture was carefully quenched with $\mathrm{H}_{2} \mathrm{O}$ at $0{ }^{\circ} \mathrm{C}$ and continuously stirred at room temperature for 30 min. The reaction mixture extracted with ethyl acetate, washed with brine, dried over $\mathrm{MgSO}_{4}$. The filtrate was concentrated under reduced pressure to give a yellowish solid residue 3 ( $R_{f}=0.13$ (ethyl acetate)). The 3 was taken forward to the next step reaction without further purication.

\section{(H) Synthesis of the 4-OHGlc-PhytoCer 4:}
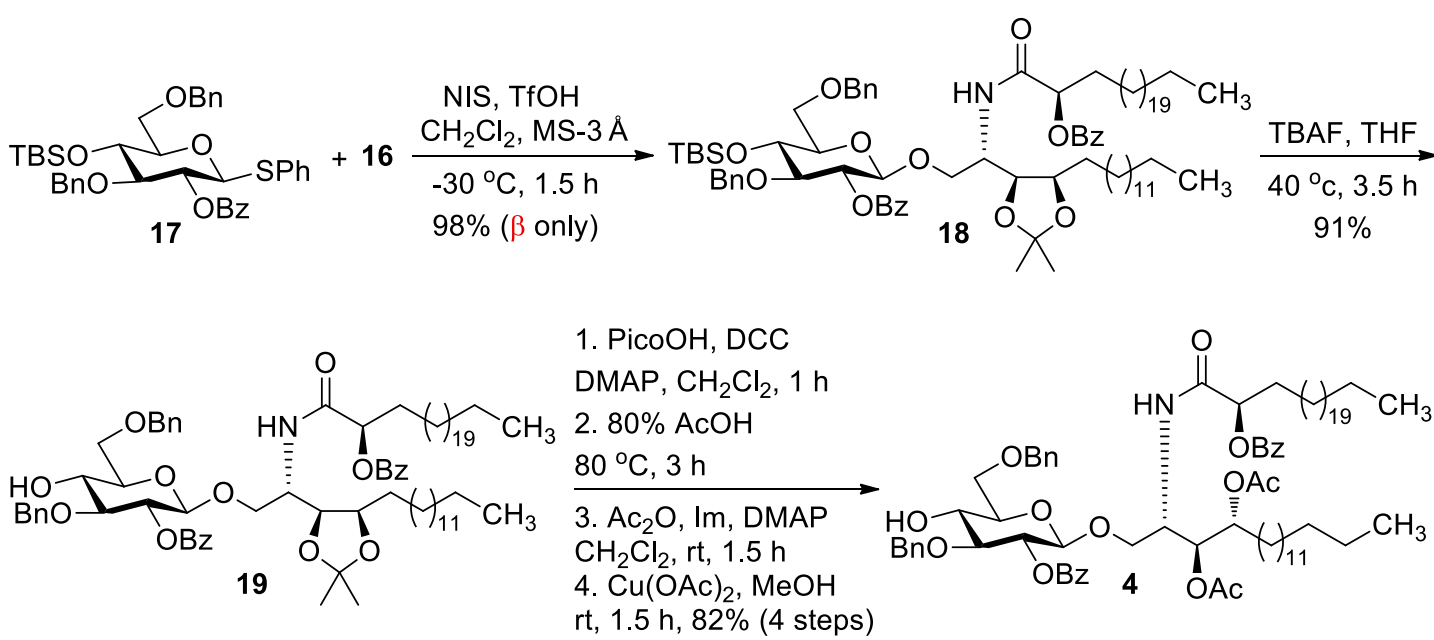

Scheme S7. Synthesis of the 4-OHGlc-PhytoCer 4.

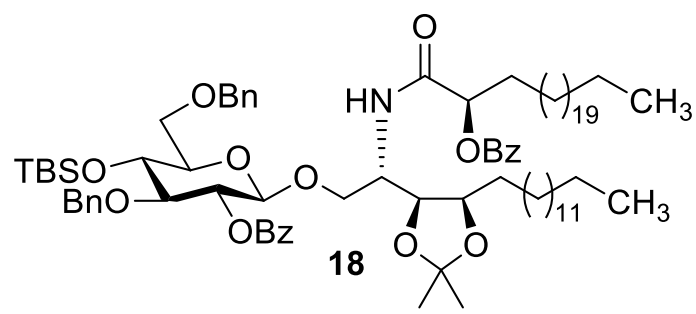


]-3,4-O-isopropylideneoctadecan-1,3,4-triol (18) A mixture of the donor 17 (0.050 g, $74.52 \mu \mathrm{mol})$, acceptor 16 (0.074 g, $89.42 \mu \mathrm{mol})$, and activated $3 \AA$ powdered molecular sieves $(0.124 \mathrm{~g})$ in anhydrous $\mathrm{CH}_{2} \mathrm{Cl}_{2}(7.5 \mathrm{~mL})$ was stirred at room temperature for $1 \mathrm{~h}$ under nitrogen to remove any trace amounts of water. The reaction mixture was then cooled to $-30{ }^{\circ} \mathrm{C}$ followed by addition of $N$-iodosuccinimide $(0.034 \mathrm{~g}, 0.15$ $\mathrm{mmol})$ and $\mathrm{TfOH}(1 \mu \mathrm{L}, 11.18 \mu \mathrm{mol})$. Continuously stirred at this temperature for $1.5 \mathrm{~h}$, the reaction mixture was carefully quenched with triethylamine and then filtered through a short pad of Celite. The filtrate was washed with cold saturated aqueous $\mathrm{Na}_{2} \mathrm{~S}_{2} \mathrm{O}_{3}$, brine, dried over $\mathrm{MgSO}_{4}$, filtered, and concentrated in vacuo. The observed yellowish syrup was purified by flash column chromatography on silica gel using ethyl acetate and hexanes $(1: 9, \mathrm{v} / \mathrm{v})$ as the eluent to give $0.103 \mathrm{~g}$ of a colorless syrup $\mathbf{1 8}$ in $98 \%$ yield: $R_{f}=0.23$ (ethyl acetate: hexanes $=1: 1(\mathrm{v} / \mathrm{v})$ ); $[\alpha]^{23}+45.5\left(\mathrm{c} 0.53, \mathrm{CHCl}_{3}\right)$; FT-IR (neat) $v_{\max } 3457,2925,2854,1729,1688,1519,1460,1368,1262,1099,847,776,707 \mathrm{~cm}^{-1} ;{ }^{1} \mathrm{H}$ NMR $(400$ $\left.\mathrm{MHz} \mathrm{CDCl}_{3}\right) \delta 8.09(\mathrm{~d}, J=8.3 \mathrm{~Hz}, 2 \mathrm{H}, \mathrm{ArH}), 7.94(\mathrm{~d}, J=8.3 \mathrm{~Hz}, 2 \mathrm{H}, \mathrm{ArH}), 7.61-7.27(\mathrm{~m}, 11 \mathrm{H}, \mathrm{ArH}), 7.14-7.09(\mathrm{~m}, 5 \mathrm{H}, \mathrm{ArH}), 6.34(\mathrm{~d}, J=8.6$ $\mathrm{Hz}, 1 \mathrm{H}, \mathrm{NH}), 5.10\left(\mathrm{t}, J=8.1 \mathrm{~Hz}, 1 \mathrm{H}, \mathrm{H}-2^{\mathrm{Glc}}\right), 4.99$ (t, $\left.J=6.3 \mathrm{~Hz}, 1 \mathrm{H}, \mathrm{H}-2^{\text {, Cer }}\right), 4.66,4.54(\mathrm{ABq}, J=12.3 \mathrm{~Hz}, 2 \mathrm{H}, \mathrm{CHaHbPh}), 4.55-4.53(\mathrm{~m}, 3 \mathrm{H}, \mathrm{H}-$ $\left.1^{\mathrm{Glc}}, \mathrm{CH}_{2} \mathrm{Ph}\right), 4.19-4.04\left(\mathrm{~m}, 3 \mathrm{H}, \mathrm{H}-3^{\mathrm{Cer}}, \mathrm{H}-2^{\mathrm{Cer}}, \mathrm{H}-1 \mathrm{a}^{\mathrm{Cer}}\right), 3.87-3.83\left(\mathrm{~m}, 1 \mathrm{H}, \mathrm{H}-4^{\mathrm{Cer}}\right), 3.73-3.66\left(\mathrm{~m}, 2 \mathrm{H}, \mathrm{H}-6 \mathrm{a}^{\mathrm{Glc}}, \mathrm{H}-1 \mathrm{~b}^{\mathrm{Cer}}\right), 3.59-3.47\left(\mathrm{~m}, 4 \mathrm{H}, \mathrm{H}-6 \mathrm{~b}^{\mathrm{Glc}}\right.$, $\left.\mathrm{H}-5^{\mathrm{Glc}}, \mathrm{H}-4^{\mathrm{Glc}}, \mathrm{H}-3^{\mathrm{Glc}}\right), 1.81-1.75\left(\mathrm{~m}, 2 \mathrm{H}, \mathrm{H}-3{ }^{\text {, Cer }}\right), 1.36\left(\mathrm{~s}, 3 \mathrm{H},\left(\mathrm{CH}_{3}\right)_{2} \mathrm{C}\right), 1.26-1.25\left(\mathrm{~m}, 69 \mathrm{H}\right.$, aliphatic), $0.88\left(\mathrm{t}, J=6.7 \mathrm{~Hz}, 6 \mathrm{H}, \mathrm{CH}_{3}\right), 0.84(\mathrm{~s}, 9 \mathrm{H}$, $\left(\mathrm{CH}_{3}\right)_{3} \mathrm{C}$ ), -0.03 (s, 3H, $\left.\mathrm{CH}_{3} \mathrm{Si}\right),-0.05$ (s, 3H, $\left.\mathrm{CH}_{3} \mathrm{Si}\right) ;{ }^{13} \mathrm{C}$ NMR $\left(100 \mathrm{MHz}, \mathrm{CDCl}_{3}\right) \delta 169.3(\mathrm{C}), 165.5(\mathrm{C}), 164.9(\mathrm{C}), 138.0(\mathrm{C}), 137.9(\mathrm{C}), 133.4$ $(\mathrm{CH}), 133.1(\mathrm{CH}), 129.9(\mathrm{CH}), 129.8(\mathrm{C}), 129.6(\mathrm{CH}), 129.6(\mathrm{C}), 128.6(\mathrm{CH}), 128.4(\mathrm{CH}), 128.0(\mathrm{CH}), 127.6(\mathrm{CH}), 127.6(\mathrm{CH}), 127.3(\mathrm{CH}), 127.2$ $(\mathrm{CH}), 107.9(\mathrm{C}), 101.0(\mathrm{CH}), 83.0(\mathrm{CH}), 77.7(\mathrm{CH}), 76.3(\mathrm{CH}), 75.2(\mathrm{CH}), 75.1(\mathrm{CH}), 74.5\left(\mathrm{CH}_{2}\right), 74.3(\mathrm{CH}), 73.5\left(\mathrm{CH}_{2}\right), 71.0\left(\mathrm{CH}^{2}, 69.2\left(\mathrm{CH}_{2}\right)\right.$, $68.7\left(\mathrm{CH}_{2}\right), 48.7(\mathrm{CH}), 31.9\left(\mathrm{CH}_{2}\right), 31.9\left(\mathrm{CH}_{2}\right), 31.6\left(\mathrm{CH}_{2}\right), 29.7(\mathrm{CH}), 29.7\left(\mathrm{CH}_{2}\right), 29.6\left(\mathrm{CH}_{2}\right), 29.5\left(\mathrm{CH}_{2}\right), 29.4\left(\mathrm{CH}_{2}\right), 29.4\left(\mathrm{CH}_{2}\right), 29.4\left(\mathrm{CH}_{2}\right)$, $29.0\left(\mathrm{CH}_{2}\right), 28.1\left(\mathrm{CH}_{3}\right), 26.6\left(\mathrm{CH}_{2}\right), 25.9\left(\mathrm{CH}_{3}\right), 25.8\left(\mathrm{CH}_{3}\right), 25.1\left(\mathrm{CH}_{2}\right), 22.7\left(\mathrm{CH}_{2}\right), 17.9(\mathrm{C}), 14.1\left(\mathrm{CH}_{3}\right) ; \mathrm{HRMS}-\mathrm{ESI}[\mathrm{M}+\mathrm{Na}]^{+} \mathrm{Calcd}$ for $\mathrm{C}_{85} \mathrm{H}_{133} \mathrm{NO}_{12} \mathrm{SiNa} 1410.9489$, Found 1410.9483 .

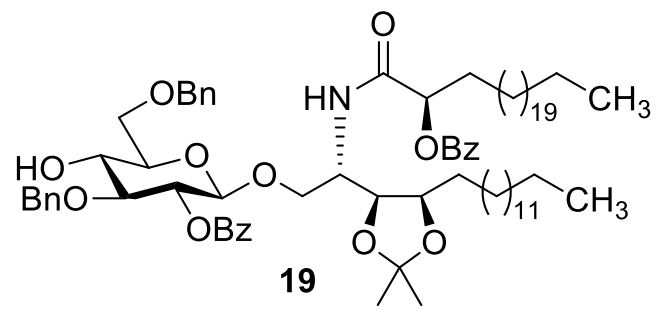

2-O-benzoyl-3,6-di- $O$-benzyl- $\beta$-D-glucopyranosyl- $(1 \rightarrow 1)-(2 S, 3 S, 4 R)$-2-[(2R)-2-(benzoyloxy)tetracosanoylamino]-3,4- $O$-isopropylideneoctadecan-1,3,4-triol (19) To a stirring solution of compound $18(0.520 \mathrm{~g}, 0.37 \mathrm{mmol})$ in THF (19 mL) was added tetra- $n$-butylammonium fluoride $(0.53 \mathrm{~mL}, 0.56 \mathrm{mmol})$ at $0{ }^{\circ} \mathrm{C}$. The mixture was warmed to $40{ }^{\circ} \mathrm{C}$ and continuously stirred at this temperature for $3.5 \mathrm{~h}$. The reaction mixture was concentrated in vасио. The resulting colorless syrup residue was purified by flash column chromatography on silica gel using ethyl acetate and hexanes $(1: 2, \mathrm{v} / \mathrm{v})$ as the eluent to get $0.435 \mathrm{~g}$ of $\mathbf{1 9}$ as a white solid compound in $91 \%$ yield: $R_{f}=0.18$ (ethyl acetate: hexanes $=1: 3(\mathrm{v} / \mathrm{v})$ ); $\mathrm{mp}=$ $101-102{ }^{\circ} \mathrm{C} ;[\alpha]^{23}{ }_{\mathrm{D}}+24.0\left(\mathrm{c} 0.35, \mathrm{CHCl}_{3}\right)$; FT-IR (neat) $v_{\max } 3443,3314,3069,2923,2853,1728,1664,1549,1459,1368,1315,1268,1174,1080$, 993, 930, 869, 741, $702 \mathrm{~cm}^{-1}$; ${ }^{1} \mathrm{H}$ NMR (400 MHz, $\left.\mathrm{CDCl}_{3}\right) \delta 8.06$ (d, J=7.7 Hz, 2H, ArH), $7.99(\mathrm{~d}, J=7.7 \mathrm{~Hz}, 2 \mathrm{H}, \operatorname{ArH}), 7.60-7.55(\mathrm{~m}, 2 \mathrm{H}, \mathrm{ArH})$, 7.48-7.42 (m, 4H, ArH), 7.38-7.30 (m, 5H, ArH), 7.20-7.16 (m, 5H, ArH), 6.26 (d, J= 8.2 Hz, 1H, NH), $5.12\left(\mathrm{t}, J=7.8 \mathrm{~Hz}, 1 \mathrm{H}, \mathrm{H}-2^{\mathrm{Glc}}\right), 4.99$ (t, $J$ $\left.=6.3 \mathrm{~Hz}, 1 \mathrm{H}, \mathrm{H}-2^{\text {,Cer }}\right), 4.65-4.54\left(\mathrm{~m}, 4 \mathrm{H}, \mathrm{CH}_{2} \mathrm{Ph}\right), 4.53\left(\mathrm{~d}, J=7.8 \mathrm{~Hz}, 1 \mathrm{H}, \mathrm{H}-1^{\mathrm{Glc}}\right), 4.15-4.06\left(\mathrm{~m}, 3 \mathrm{H}, \mathrm{H}-3^{\mathrm{Cer}}, \mathrm{H}-2^{\mathrm{Cer}}, \mathrm{H}-1 \mathrm{a}^{\mathrm{Cer}}\right), 3.87-3.83(\mathrm{~m}, 1 \mathrm{H}$, $\left.\mathrm{H}-4^{\mathrm{Cer}}\right), 3.74-3.64\left(\mathrm{~m}, 4 \mathrm{H}, \mathrm{H}-6 \mathrm{a}^{\mathrm{Glc}}, \mathrm{H}-5^{\mathrm{Glc}}, \mathrm{H}-4^{\mathrm{Glc}}, \mathrm{H}-1 \mathrm{a}^{\mathrm{Cer}}\right), 3.57-3.50\left(\mathrm{~m}, 2 \mathrm{H}, \mathrm{H}-6 \mathrm{~b}^{\mathrm{Glc}}, \mathrm{H}-3^{\mathrm{Glc}}\right), 2.71\left(\mathrm{~d}, J=1.5 \mathrm{~Hz}, 1 \mathrm{H}, \mathrm{OH}-4^{\mathrm{Glc}}\right), 1.80-1.74(\mathrm{~m}, 2 \mathrm{H}$, $\left.\mathrm{H}-3{ }^{,}{ }^{\mathrm{Cer}}\right), 1.35\left(\mathrm{~s}, 3 \mathrm{H},\left(\mathrm{CH}_{3}\right)_{2} \mathrm{C}\right), 1.25-1.23\left(\mathrm{~m}, 69 \mathrm{H},\left(\mathrm{CH}_{3}\right)_{2} \mathrm{C}\right.$, aliphatic), $0.88\left(\mathrm{t}, J=6.7 \mathrm{~Hz}, 6 \mathrm{H}, \mathrm{CH}_{3}\right) ;{ }^{13} \mathrm{C} \mathrm{NMR}(100 \mathrm{MHz}, \mathrm{CDCl}) \delta 169.4(\mathrm{C})$, $165.6(\mathrm{C}), 165.1(\mathrm{C}), 138.0(\mathrm{C}), 137.7(\mathrm{C}), 133.5(\mathrm{CH}), 133.3(\mathrm{CH}), 129.9(\mathrm{CH}), 129.8(\mathrm{C}), 129.8(\mathrm{CH}), 129.5(\mathrm{C}), 128.6(\mathrm{CH}), 128.6(\mathrm{CH}), 128.4$ $(\mathrm{CH}), 128.0(\mathrm{CH}), 127.9(\mathrm{CH}), 127.8(\mathrm{CH}), 127.8(\mathrm{CH}), 108.0(\mathrm{C}), 101.1(\mathrm{CH}), 82.1(\mathrm{CH}), 77.7(\mathrm{CH}), 75.3(\mathrm{CH}), 74.9(\mathrm{CH}), 74.4(\mathrm{CH}), 74.3$ $(\mathrm{CH}), 73.8\left(\mathrm{CH}_{2}\right), 73.7(\mathrm{CH}), 72.0(\mathrm{CH}), 70.3\left(\mathrm{CH}_{2}\right), 68.7\left(\mathrm{CH}_{2}\right), 48.8(\mathrm{CH}), 32.0\left(\mathrm{CH}_{2}\right), 32.0\left(\mathrm{CH}_{2}\right), 31.6\left(\mathrm{CH}_{2}\right), 29.8\left(\mathrm{CH}_{2}\right), 29.8\left(\mathrm{CH}_{2}\right), 29.7$ $\left(\mathrm{CH}_{2}\right), 29.7\left(\mathrm{CH}_{2}\right), 29.6\left(\mathrm{CH}_{2}\right), 29.6\left(\mathrm{CH}_{2}\right), 29.5\left(\mathrm{CH}_{2}\right), 29.4\left(\mathrm{CH}_{2}\right), 29.2\left(\mathrm{CH}_{2}\right), 28.1\left(\mathrm{CH}_{3}\right), 26.6\left(\mathrm{CH}_{2}\right), 25.8\left(\mathrm{CH}_{3}\right), 25.0\left(\mathrm{CH}_{2}\right), 22.8\left(\mathrm{CH}_{2}\right), 14.2$ $\left(\mathrm{CH}_{3}\right)$; HRMS-ESI $[\mathrm{M}+\mathrm{Na}]^{+} \mathrm{Calcd}$ for $\mathrm{C}_{79} \mathrm{H}_{119} \mathrm{NO}_{12} \mathrm{Na} 1296.8624$, Found 1296.8623 .

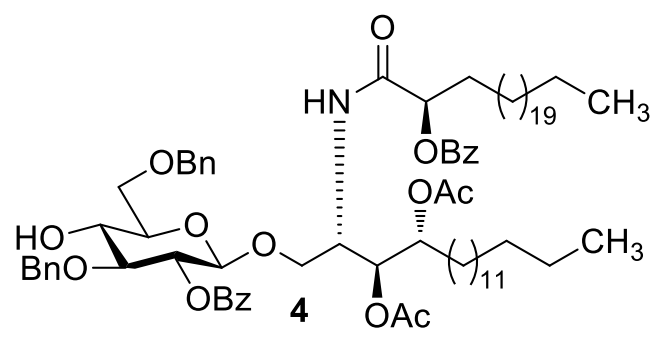


1,3,4-triol (4) To a stirring solution of compound $19(0.101 \mathrm{~g}, 78.43 \mu \mathrm{mol})$ in dry dichloroethane $(0.8 \mathrm{~mL})$ was added 2-picolinic acid $(0.012 \mathrm{~g}$, $94.12 \mu \mathrm{mol})$, DMAP $(0.001 \mathrm{~g}, 7.84 \mu \mathrm{mol})$ and DCC $(0.025 \mathrm{~mL}, 0.16 \mathrm{mmol})$ at $0{ }^{\circ} \mathrm{C}$. The mixture was warmed to room temperature and continuously stirred at this temperature for $1 \mathrm{~h}$. The reaction mixture was filtered through a short pad of Celite and concentrated in vacuo. The resulting compound $\left(R_{f}=0.95\right.$ (ethyl acetate: hexanes $\left.=1: 1(\mathrm{v} / \mathrm{v})\right)$ was dissolved in a solution of $80 \%$ aqueous $\mathrm{AcOH}(1.6 \mathrm{~mL})$ at $0{ }^{\circ} \mathrm{C}$ and then the mixture was continuously stirred for $3 \mathrm{~h}$ at $80{ }^{\circ} \mathrm{C}$. The reaction mixture was co-evaporated with toluene to give yellowish solid residue. The resulting compound $\left(R_{f}=0.20\right.$ (ethyl acetate: hexanes $\left.=1: 1(\mathrm{v} / \mathrm{v})\right)$ was dissolved in dry dichloroethane $(0.8 \mathrm{~mL})$ and was added $\operatorname{Im}(0.013 \mathrm{~g}, 0.20$ mmol), DMAP $(0.001 \mathrm{~g}, 7.84 \mu \mathrm{mol})$, and andacetic anhydride $(0.018 \mathrm{~mL}, 0.20 \mathrm{mmol})$ at $0{ }^{\circ} \mathrm{C}$. The mixture was warmed to room temperature and continuously stirred at this temperature for $1.5 \mathrm{~h}$. The reaction mixture was carefully quenched with $\mathrm{MeOH}$ and extracted with ethyl acetate, washed with cold $1 \mathrm{~N} \mathrm{HCl}$, brine, dried over $\mathrm{MgSO}_{4}$, and concentrated in vacuo. The resulting compound $\left(R_{f}=0.18\right.$ (ethyl acetate: hexanes $=1: 1$ $(\mathrm{v} / \mathrm{v}))$ was dissolved in $\mathrm{MeOH}(0.8 \mathrm{~mL})$ and was added $\mathrm{Cu}(\mathrm{OAc})_{2}(0.017 \mathrm{~g}, 94.11 \mu \mathrm{mol})$ at $0{ }^{\circ} \mathrm{C}$. The mixture was warmed to room temperature and continuously stirred at this temperature for $1.5 \mathrm{~h}$. The reaction mixture was extracted with ethyl acetate, washed with cold $1 \mathrm{~N} \mathrm{HCl}$, brine, dried over $\mathrm{MgSO} 4$, and concentrated in vacuo. The observed brown solid was purified by flash column chromatography on silica gel using ethyl acetate and hexanes $(1: 3, \mathrm{v} / \mathrm{v})$ as the eluent to give $0.084 \mathrm{~g}$ of a white solid 4 in $82 \%$ yield: $R_{f}=0.18$ (ethyl acetate: hexanes $\left.=1: 3(\mathrm{v} / \mathrm{v})\right) ;[\alpha]^{29} \mathrm{D}+12.3(\mathrm{c}$ $0.40, \mathrm{CHCl}_{3}$ ); FT-IR (neat) $v_{\max } 3425,2924,2853,1730,1690,1521,1454,1368,1267,1226,1071,1027,711 \mathrm{~cm}^{-1} ;{ }^{1} \mathrm{H} \mathrm{NMR}\left(400 \mathrm{MHz}, \mathrm{CDCl}_{3}\right)$ $\delta 8.07(\mathrm{~d}, J=7.6 \mathrm{~Hz}, 2 \mathrm{H}, \mathrm{ArH}), 7.92(\mathrm{~d}, J=7.5 \mathrm{~Hz}, 2 \mathrm{H}, \mathrm{ArH}), 7.60-7.30(\mathrm{~m}, 11 \mathrm{H}, \mathrm{ArH}), 7.17-7.13(\mathrm{~m}, 5 \mathrm{H}, \mathrm{ArH}), 6.82(\mathrm{~d}, J=9.0 \mathrm{~Hz}, 1 \mathrm{H}, \mathrm{NH})$, 5.17-5.13 (m, 2H, H-3 $\left.{ }^{\mathrm{Cer}}, \mathrm{H}-2^{\text {,Cer }}\right), 5.06$ (t, $\left.J=8.6 \mathrm{~Hz}, 1 \mathrm{H}, \mathrm{H}-2^{\mathrm{Glc}}\right), 4.97$ (dt, $\left.J=10.2,2.6 \mathrm{~Hz}, 1 \mathrm{H}, \mathrm{H}-4^{\mathrm{Cer}}\right), 4.63\left(\mathrm{~s}, 2 \mathrm{H}, \mathrm{CH}_{2} \mathrm{Ph}\right), 4.57,4.52(\mathrm{ABq}, J$ $=11.9 \mathrm{~Hz}, 2 \mathrm{H}, \mathrm{CHaHbPh}), 4.46\left(\mathrm{~d}, J=8.0 \mathrm{~Hz}, 1 \mathrm{H}, \mathrm{H}-1^{\mathrm{Glc}}\right), 4.32-4.26\left(\mathrm{~m}, 1 \mathrm{H}, \mathrm{H}-2^{\mathrm{Cer}}\right), 3.80\left(\mathrm{dd}, J=10.5,3.6 \mathrm{~Hz}, 1 \mathrm{H}, \mathrm{H}-1 \mathrm{a}^{\mathrm{Cer}}\right), 3.72-3.61(\mathrm{~m}, 4 \mathrm{H}$, $\left.\mathrm{H}-6 \mathrm{a}^{\mathrm{Glc}}, \mathrm{H}-6 \mathrm{~b}^{\mathrm{Glc}}, \mathrm{H}-4^{\mathrm{Glc}}, \mathrm{H}-1 \mathrm{~b}^{\mathrm{Cer}}\right), 3.55\left(\mathrm{t}, J=9.1 \mathrm{~Hz}, 1 \mathrm{H}, \mathrm{H}-3^{\mathrm{Glc}}\right), 3.38\left(\mathrm{dt}, J=9.5,4.7 \mathrm{~Hz}, 1 \mathrm{H}, \mathrm{H}-5^{\mathrm{Glc}}\right), 1.97$ (s, 3H, Ac), $1.94(\mathrm{~s}, 3 \mathrm{H}, \mathrm{Ac}), 1.86-$ $1.80\left(\mathrm{~m}, 2 \mathrm{H}, \mathrm{H}-3{ }^{\text {,Cer }}\right), 1.65-1.49\left(\mathrm{~m}, 2 \mathrm{H}, \mathrm{H}-5^{\mathrm{Cer}}\right), 1.25-1.21\left(\mathrm{~m}, 64 \mathrm{H}\right.$, aliphatic), $0.88\left(\mathrm{t}, J=6.7 \mathrm{~Hz}, 6 \mathrm{H}, \mathrm{CH}_{3}\right) ;{ }^{13} \mathrm{C} \mathrm{NMR}\left(100 \mathrm{MHz} \mathrm{CDCl}_{3}\right) \delta 170.8$ (C), 169.9 (C), 169.8 (C), 165.3 (C), $165.2(\mathrm{C}), 138.0(\mathrm{C}), 137.7$ (C), $133.5(\mathrm{CH}), 133.3(\mathrm{CH}), 130.0(\mathrm{CH}), 129.8(\mathrm{CH}), 129.7(\mathrm{C}), 129.6(\mathrm{C}), 128.6$ $(\mathrm{CH}), 128.5(\mathrm{CH}), 128.4(\mathrm{CH}), 128.0(\mathrm{CH}), 127.9(\mathrm{CH}), 127.8(\mathrm{CH}), 127.8(\mathrm{CH}), 100.1(\mathrm{CH}), 82.1(\mathrm{CH}), 74.5(\mathrm{CH}), 74.5(\mathrm{CH}), 73.9(\mathrm{CH}), 73.8$ $\left(\mathrm{CH}_{2}\right), 73.2(\mathrm{CH}), 72.9(\mathrm{CH}), 72.2(\mathrm{CH}), 72.0(\mathrm{CH}), 70.4\left(\mathrm{CH}_{2}\right), 65.6\left(\mathrm{CH}_{2}\right), 47.8(\mathrm{CH}), 32.0\left(\mathrm{CH}_{2}\right), 31.8\left(\mathrm{CH}_{2}\right), 29.8\left(\mathrm{CH}_{2}\right), 29.8\left(\mathrm{CH}_{2}\right), 29.8$ $\left(\mathrm{CH}_{2}\right), 29.7\left(\mathrm{CH}_{2}\right), 29.7\left(\mathrm{CH}_{2}\right), 29.5\left(\mathrm{CH}_{2}\right), 29.5\left(\mathrm{CH}_{2}\right), 29.5\left(\mathrm{CH}_{2}\right), 29.5\left(\mathrm{CH}_{2}\right), 29.4\left(\mathrm{CH}_{2}\right), 28.4\left(\mathrm{CH}_{2}\right), 25.7\left(\mathrm{CH}_{2}\right), 24.8\left(\mathrm{CH}_{2}\right), 22.8\left(\mathrm{CH}_{2}\right), 21.1$ $\left(\mathrm{CH}_{3}\right), 20.7\left(\mathrm{CH}_{3}\right), 14.2\left(\mathrm{CH}_{3}\right)$; HRMS-ESI [M + Na] $]^{+}$Calcd for $\mathrm{C}_{80} \mathrm{H}_{11} \mathrm{NO}_{14} \mathrm{Na} 1340.8523$, Found 1340.8546. 
(I) Completion of the total synthesis of the ganglioside LLG-3 (1):

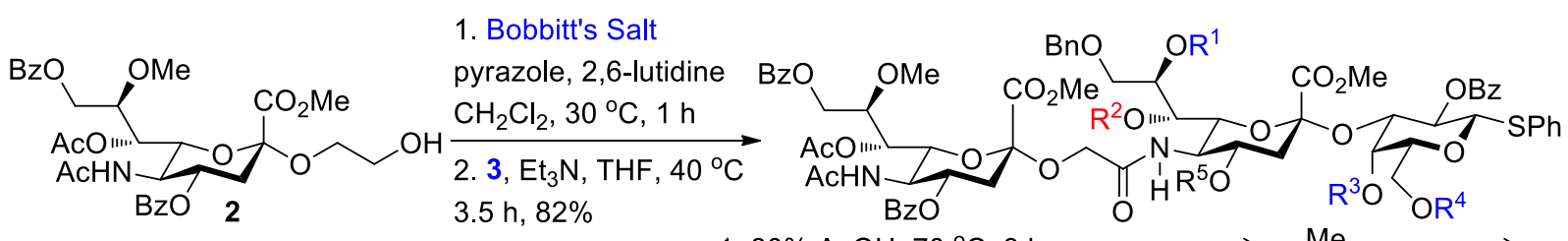

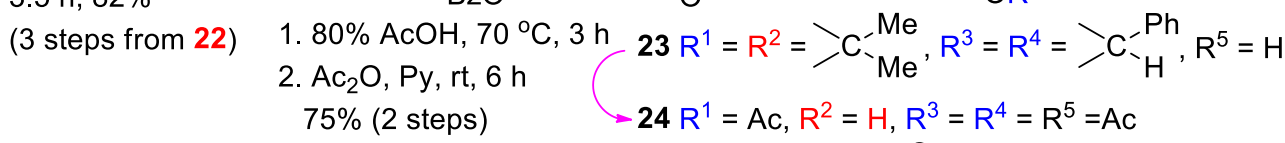

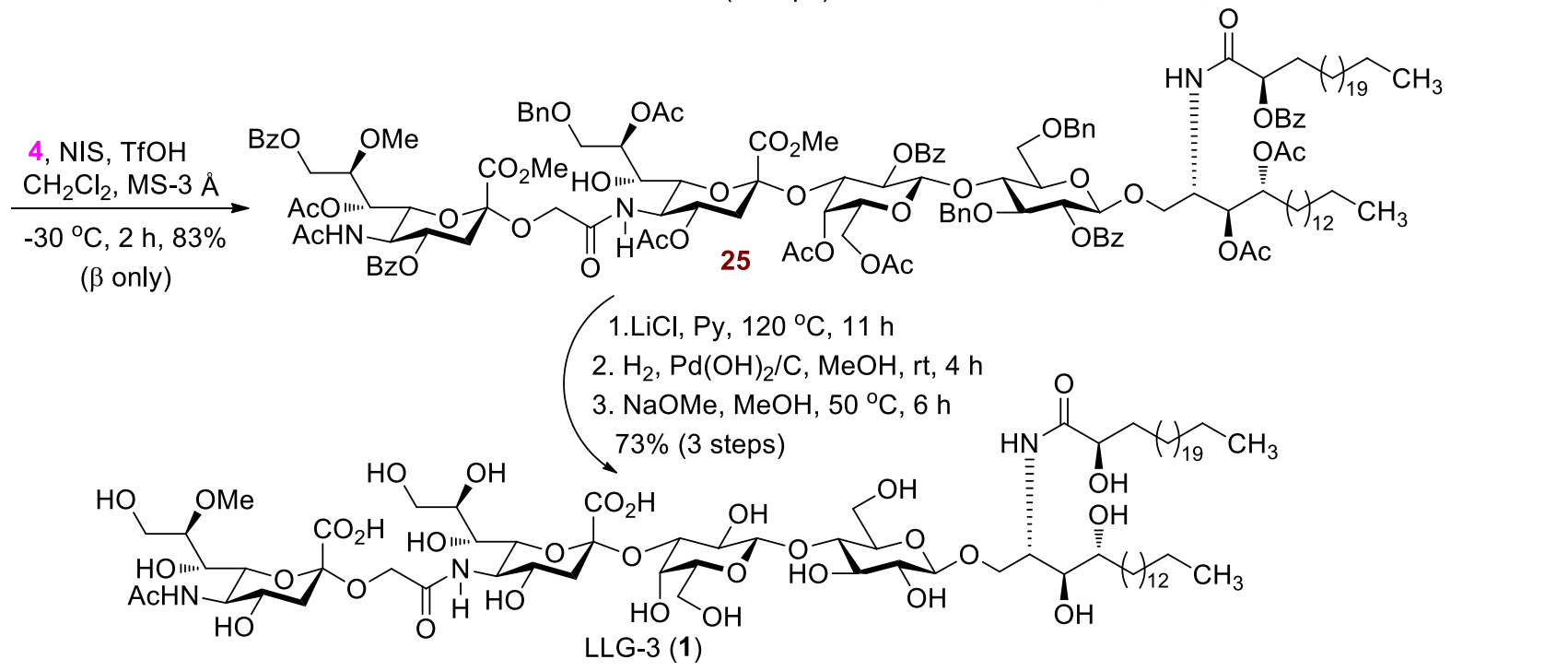

Scheme 6. Completion of the total synthesis of the ganglioside LLG-3 (1).

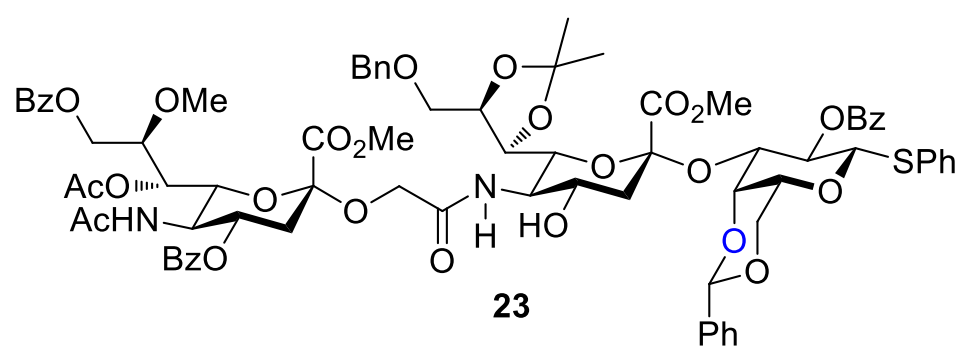

Phenyl (methyl 5-acetamido-7-O-acetyl-4,9-di-O-benzoyl-3,5-dideoxy-8-O-methyl-D-glycero- $\alpha$-D-galacto-2-nonulopyranosylonate)-(2 $\rightarrow 11$ )methyl 9-O-benzyl-3,5-dideoxy-5-glycolylamido-7,8- $O$-isopropylidene-D-glycero- $\alpha$-D-galacto-2-nonulopyranosylonate-(2 $\rightarrow 3$ )-2- $O$-benzoyl4,6-O-benzylidene-1-thio-p-D-galactopyranoside (23) To a stirring solution of compound 2 (0.103 g, $0.16 \mathrm{mmol})$ and pyrazole $(0.013 \mathrm{~g}, 0.20$ $\mathrm{mmol})$ in $\mathrm{CH}_{2} \mathrm{Cl}_{2}(3.3 \mathrm{~mL})$ was added 2,6-lutidine $(0.087 \mathrm{~mL}, 0.73 \mathrm{mmol})$ and 4-acetamido-2,2,6,6-tetramethylpiperidine-1-oxoammonium tetrafluoroborate $(0.244 \mathrm{~mL}, 0.81 \mathrm{mmol})$ at $0{ }^{\circ} \mathrm{C}$. The mixture was warmed to $30{ }^{\circ} \mathrm{C}$ and continuously stirred at this temperature for $1 \mathrm{~h}$. After this time, $\mathrm{Et}_{2} \mathrm{O}$ was added, causing precipitation of the nitroxide. The mixture was filtered and the filter was washed with $\mathrm{Et}_{2} \mathrm{O}$. $\mathrm{The}$ filtrate was extracted with $\mathrm{Et}_{2} \mathrm{O}$, washed with $1 \mathrm{~N} \mathrm{HCl}$, brine, dried over $\mathrm{MgSO}_{4}$, and concentrated in vacuo. The resulting compound $\left(R_{f}=0.45\right.$ (ethyl acetate:hexanes $=2: 1(\mathrm{v} / \mathrm{v})))$ and $\mathbf{3}(0.155 \mathrm{~g}, 0.18 \mathrm{mmol})$ was dissolved in THF $(0.8 \mathrm{~mL})$ was added triethylamine $(0.11 \mathrm{~mL}, 0.82 \mathrm{mmol})$ at $0{ }^{\circ} \mathrm{C}$. The mixture was warmed to $40{ }^{\circ} \mathrm{C}$ and continuously stirred at this temperature for $3.5 \mathrm{~h}$. The reaction mixture was extracted with ethyl acetate, washed with brine, dried over $\mathrm{MgSO}_{4}$, and concentrated in vacuo. The resulting yellowish syrup residue was purified by flash column chromatography on silica gel using ethyl acetate and hexanes $(2: 1, \mathrm{v} / \mathrm{v})$ as the eluent to get $0.200 \mathrm{~g}$ of $\mathbf{2 3}$ as a white solid compound in $82 \%$ yield: $R_{f}=0.13$ (ethyl acetate:hexanes $\left.=2: 1(\mathrm{v} / \mathrm{v})\right) ; \mathrm{mp}=132-133{ }^{\circ} \mathrm{C} ;[\alpha]^{23}{ }_{\mathrm{D}}+3.4\left(\mathrm{c} 0.46, \mathrm{CHCl}_{3}\right) ;$ FT-IR (neat) $v_{\max } 3365,2999,2942,1736,1594,1533$, 1447, 1373, 1275, 1211, 1103, 1035, 868, 814, 753, $710 \mathrm{~cm}^{-1}$; ${ }^{1} \mathrm{H}$ NMR (400 MHz, $\left.\mathrm{CDCl}_{3}\right) \delta$ 8.05-7.95 (m, 6H, ArH), 7.60-7.20 (m, 24H, ArH), $6.35\left(\mathrm{~d}, J=8.3 \mathrm{~Hz}, 1 \mathrm{H}, \mathrm{NH}^{\mathrm{NeuB}}\right), 5.51\left(\mathrm{t}, J=10.0 \mathrm{~Hz}, 1 \mathrm{H}, \mathrm{H}-2^{\mathrm{Gal}}\right), 5.42(\mathrm{~s}, 1 \mathrm{H}, \mathrm{CHPh}), 5.31-5.24\left(\mathrm{~m}, 2 \mathrm{H}, \mathrm{NH}^{\mathrm{NeuA}}, \mathrm{H}^{7^{\mathrm{NeuA}}}\right), 5.17(\mathrm{td}, J=9.9,5.1$ $\left.\mathrm{Hz}, 1 \mathrm{H}, \mathrm{H}-4^{\mathrm{NeuA}}\right), 4.76\left(\mathrm{~d}, J=10.0 \mathrm{~Hz}, 1 \mathrm{H}, \mathrm{H}-1^{\mathrm{Gal}}\right), 4.72\left(\mathrm{dd}, J=12.2,2.4 \mathrm{~Hz}, 1 \mathrm{H}, \mathrm{H}-9 \mathrm{a}^{\mathrm{NeuA}}\right), 4.60,4.53(\mathrm{ABq}, J=12.1 \mathrm{~Hz}, 2 \mathrm{H}, \mathrm{CHaHbPh}), 4.54-$ $4.48\left(\mathrm{~m}, 3 \mathrm{H}, \mathrm{H}-8^{\mathrm{NeuB}}, \mathrm{H}-4^{\mathrm{Gal}}, \mathrm{H}-3^{\mathrm{Gal}}\right), 4.37-4.24\left(\mathrm{~m}, 4 \mathrm{H}, \mathrm{H}-6^{\mathrm{NeuA}}, \mathrm{H}-6 \mathrm{a}^{\mathrm{Gal}}, \mathrm{H}-5^{\mathrm{NeuA}}, \mathrm{OCHaHbCO}\right), 4.18-4.09\left(\mathrm{~m}, 3 \mathrm{H}, \mathrm{H}-9 \mathrm{~b}^{\mathrm{NeuA}}, \mathrm{H}-7^{\mathrm{NeuB}}\right.$, OCHaHbCO), 4.04, $3.98\left(\mathrm{ABX}, J_{\mathrm{AB}}=10.3 \mathrm{~Hz}, J_{\mathrm{AX}}=5.0 \mathrm{~Hz}, J_{\mathrm{BX}}=6.9 \mathrm{~Hz}, 2 \mathrm{H}, \mathrm{H}-9 \mathrm{a}^{\mathrm{NeuB}}, \mathrm{H}_{-}-9 \mathrm{~b}^{\mathrm{NeuB}}\right), 3.89-3.80\left(\mathrm{~m}, 4 \mathrm{H}, \mathrm{H}-8^{\mathrm{NeuA}}, \mathrm{H}-6^{\mathrm{NeuB}}, \mathrm{H}-6 \mathrm{~b}^{\mathrm{Gal}}\right.$, 
$\left.\mathrm{H}-5^{\mathrm{NeuB}}\right), 3.85\left(\mathrm{~s}, 3 \mathrm{H}, \mathrm{CO}_{2} \mathrm{CH}_{3}{ }^{\mathrm{NeuA}}\right), 3.55-3.53\left(\mathrm{~m}, 1 \mathrm{H}, \mathrm{H}-4^{\mathrm{NeuB}}\right), 3.50\left(\mathrm{~s}, 3 \mathrm{H}, \mathrm{OCH}_{3}\right), 3.43\left(\mathrm{~s}, 1 \mathrm{H}, \mathrm{H}-5^{\mathrm{Gal}}\right), 3.34\left(\mathrm{~s}, 3 \mathrm{H}, \mathrm{CO}_{2} \mathrm{CH}_{3}{ }^{\mathrm{NeuB}}\right), 3.10(\mathrm{~d}, J=$ $4.2 \mathrm{~Hz}, 1 \mathrm{H}, \mathrm{OH}), 2.86\left(\mathrm{dd}, J=12.9,5.1 \mathrm{~Hz}, 1 \mathrm{H}, \mathrm{H}-3 \mathrm{eq}^{\mathrm{NeuA}}\right), 2.45\left(\mathrm{dd}, J=12.9,4.3 \mathrm{~Hz}, 1 \mathrm{H}, \mathrm{H}-3 \mathrm{eq}^{\mathrm{NeuB}}\right), 2.10-2.04\left(\mathrm{~m}, 1 \mathrm{H}, \mathrm{H}-3 \mathrm{ax}^{\mathrm{NeuA}}\right), 2.07(\mathrm{~s}, 3 \mathrm{H}$, $\mathrm{Ac}), 1.77(\mathrm{~s}, 3 \mathrm{H}, \mathrm{Ac}), 1.58\left(\mathrm{~s}, 3 \mathrm{H},\left(\mathrm{CH}_{3}\right)_{2} \mathrm{C}\right), 1.55-1.52\left(\mathrm{~m}, 1 \mathrm{H}, \mathrm{H}-3 \mathrm{ax}^{\mathrm{NeuB}}\right), 1.39\left(\mathrm{~s}, 3 \mathrm{H},\left(\mathrm{CH}_{3}\right)_{2} \mathrm{C}\right) ;{ }^{13} \mathrm{C} \mathrm{NMR}\left(100 \mathrm{MHz}, \mathrm{CDCl}_{3}\right) \delta 170.5(\mathrm{C}), 169.9$ (C), $169.8(\mathrm{C}), 168.8(\mathrm{C}), 167.8(\mathrm{C}), 166.4(\mathrm{C}), 166.3(\mathrm{C}), 164.8(\mathrm{C}), 137.6(\mathrm{C}), 137.5(\mathrm{C}), 133.6(\mathrm{CH}), 133.4(\mathrm{CH}), 133.1(\mathrm{CH}), 133.1(\mathrm{CH}), 131.7$ $(\mathrm{C}), 129.7(\mathrm{C}), 129.6(\mathrm{CH}), 129.6(\mathrm{C}), 129.0(\mathrm{CH}), 128.9(\mathrm{C}), 128.6(\mathrm{C}), 128.4(\mathrm{CH}), 128.4(\mathrm{CH}), 128.0(\mathrm{CH}), 127.8(\mathrm{CH}), 127.8(\mathrm{CH}), 126.4$ $(\mathrm{CH}), 108.6(\mathrm{C}), 100.7(\mathrm{CH}), 98.6(\mathrm{C}), 98.1(\mathrm{C}), 85.8(\mathrm{CH}), 76.5(\mathrm{CH}), 76.3(\mathrm{CH}), 74.0(\mathrm{CH}), 73.9(\mathrm{CH}), 73.2(\mathrm{CH}), 72.9\left(\mathrm{CH}_{2}\right), 72.6(\mathrm{CH}), 72.6$ $(\mathrm{CH}), 70.5(\mathrm{CH}), 69.5(\mathrm{CH}), 69.1\left(\mathrm{CH}_{2}\right), 68.7\left(\mathrm{CH}_{2}\right), 68.2(\mathrm{CH}), 67.9(\mathrm{CH}), 67.7(\mathrm{CH}), 63.6\left(\mathrm{CH}_{2}\right), 62.6\left(\mathrm{CH}_{2}\right), 58.6\left(\mathrm{CH}_{3}\right), 53.0\left(\mathrm{CH}_{3}\right), 53.0(\mathrm{CH})$, $52.3\left(\mathrm{CH}_{3}\right), 48.1(\mathrm{CH}), 39.8\left(\mathrm{CH}_{2}\right), 37.5\left(\mathrm{CH}_{2}\right), 27.0\left(\mathrm{CH}_{3}\right), 24.8\left(\mathrm{CH}_{3}\right), 22.9\left(\mathrm{CH}_{3}\right), 20.7\left(\mathrm{CH}_{3}\right) ; \mathrm{HRMS}-\mathrm{ESI}[\mathrm{M}+\mathrm{Na}]^{+} \mathrm{Calcd}$ for $\mathrm{C}_{77} \mathrm{H}_{84} \mathrm{~N}_{2} \mathrm{O}_{26} \mathrm{SNa}$ 1507.4925, Found 1507.4932.

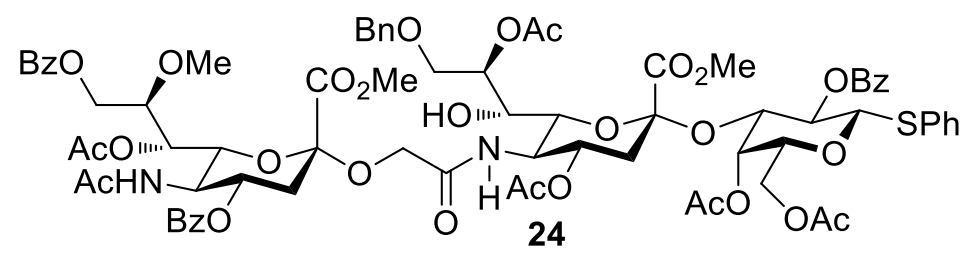

Phenyl (methyl 5-acetamido-7- $O$-acetyl-4,9-di- $O$-benzoyl-3,5-dideoxy-8- $O$-methyl-D-glycero- $\alpha$-D-galacto-2-nonulopyranosylonate)-(2 $\rightarrow 11$ )(methyl 9-O-benzyl-4,8-di- $O$-acetyl-3,5-dideoxy-5-glycolylamido-D-glycero-a-D-galacto-2-nonulopyranosylonate)-(2 $\rightarrow$ 3)-2-O-benzoyl-4,6di- $\boldsymbol{O}$-acetyl-1-thio- $\boldsymbol{\beta}$-D-galactopyranoside (24) The isopropylidene acetal $23(0.041 \mathrm{~g}, 26.92 \mu \mathrm{mol})$ was dissolved in a solution of $80 \%$ aqueous $\mathrm{AcOH}(0.9 \mathrm{~mL})$ at $0{ }^{\circ} \mathrm{C}$ and then the mixture was continuously stirred for $3 \mathrm{~h}$ at $70{ }^{\circ} \mathrm{C}$. The reaction mixture was co-evaporated with toluene to give a white solid residue. The resulting compound $\left(R_{f}=0.40\right.$ (ethyl acetate: hexanes $\left.=3: 1(\mathrm{v} / \mathrm{v})\right)$ was dissolved in pyridine $(0.9 \mathrm{~mL})$ was added acetic anhydride $(0.019 \mathrm{~mL}, 0.20 \mathrm{mmol})$ at $0{ }^{\circ} \mathrm{C}$. The mixture was warmed to room temperature and continuously stirred at this temperature for $6 \mathrm{~h}$. The reaction mixture was carefully quenched with $\mathrm{MeOH}$ and extracted with ethyl acetate, washed with cold $1 \mathrm{~N} \mathrm{HCl}$, brine, dried over $\mathrm{MgSO}_{4}$, and concentrated in vасио. The resulting yellowish syrup residue was purified by flash column chromatography on silica gel using ethyl acetate and hexanes $(2: 1, \mathrm{v} / \mathrm{v})$ as the eluent to get $0.030 \mathrm{~g}$ of $\mathbf{2 4}$ as a white solid compound in $75 \%$ yield: $R_{f}=0.38$ (ethyl acetate: hexanes $=3: 1(\mathrm{v} / \mathrm{v})$ ); $[\alpha]^{29}+28.6\left(\mathrm{c} 0.18, \mathrm{CHCl}_{3}\right)$; FT-IR (neat) $v_{\max } 3366,2953,2929,1745,1665,1539,1451,1371,1269,1234,1097,1034,714 \mathrm{~cm}^{-1} ;{ }^{1} \mathrm{H} \mathrm{NMR}(400$ $\left.\mathrm{MHz}, \mathrm{CDCl}_{3}\right) \delta 8.22(\mathrm{~d}, J=7.6 \mathrm{~Hz}, 2 \mathrm{H}, \mathrm{ArH}), 8.06(\mathrm{~d}, J=7.6 \mathrm{~Hz}, 2 \mathrm{H}, \mathrm{ArH}), 7.98(\mathrm{~d}, J=7.6 \mathrm{~Hz}, 2 \mathrm{H}, \mathrm{ArH}), 7.58-7.51(\mathrm{~m}, 3 \mathrm{H}, \mathrm{ArH}), 7.48-7.39(\mathrm{~m}$, $8 \mathrm{H}, \mathrm{ArH}), 7.33-7.22(\mathrm{~m}, 8 \mathrm{H}, \mathrm{ArH}), 6.76\left(\mathrm{~d}, J=8.0 \mathrm{~Hz}, 1 \mathrm{H}, \mathrm{NH}^{\mathrm{NeuB}}\right), 5.46-5.44\left(\mathrm{~m}, 1 \mathrm{H}, \mathrm{H}-8^{\mathrm{NeuB}}\right), 5.38\left(\mathrm{t}, J=10.0 \mathrm{~Hz}, 1 \mathrm{H}, \mathrm{H}-2^{\mathrm{Gal}}\right), 5.30-5.26(\mathrm{~m}$, $\left.2 \mathrm{H}, \mathrm{H}-7^{\mathrm{NeuA}}, \mathrm{NH}^{\mathrm{NeuA}}\right), 5.22-5.15\left(\mathrm{~m}, 1 \mathrm{H}, \mathrm{H}_{-}{ }^{\mathrm{NeuA}}\right), 5.11\left(\mathrm{~d}, J=2.1 \mathrm{~Hz}, 1 \mathrm{H}, \mathrm{H}-4^{\mathrm{Gal}}\right), 5.02\left(\mathrm{~d}, J=10.1 \mathrm{~Hz}, 1 \mathrm{H}, \mathrm{H}-1^{\mathrm{Gal}}\right), 4.99-4.92\left(\mathrm{~m}, 2 \mathrm{H}, \mathrm{H}-4^{\mathrm{NeuB}}\right.$, $\left.\mathrm{H}-3^{\mathrm{Gal}}\right), 4.73\left(\mathrm{dd}, J=12.3,2.2 \mathrm{~Hz}, 1 \mathrm{H}, \mathrm{H}-9 \mathrm{a}^{\mathrm{NeuA}}\right), 4.66\left(\mathrm{~d}, J=4.6 \mathrm{~Hz}, 1 \mathrm{H}, \mathrm{OH}-7^{\mathrm{NeuB}}\right), 4.56,4.51(\mathrm{ABq}, J=12.2 \mathrm{~Hz}, 2 \mathrm{H}, \mathrm{CH} a \mathrm{HbPh}), 4.37(\mathrm{AB}, J=$ $15.4 \mathrm{~Hz}, 1 \mathrm{H}, \mathrm{OCHaHbCO}), 4.27$ (q, $\left.J=10.1 \mathrm{~Hz}, 1 \mathrm{H}, \mathrm{H}-5^{\mathrm{NeuA}}\right), 4.20$ (d, $\left.J=10.8 \mathrm{~Hz}, 1 \mathrm{H}, \mathrm{H}-6^{\mathrm{NeuA}}\right), 4.12-4.08$ (m, 3H, H-9a $\left.{ }^{\mathrm{NeuA}}, \mathrm{H}-5^{\mathrm{Gal}}, \mathrm{H}-6^{\mathrm{Gal}}\right)$, $4.02(\mathrm{AB}, J=15.4 \mathrm{~Hz}, 1 \mathrm{H}, \mathrm{OCHa} H b \mathrm{CO}), 3.87-3.78$ (m, 3H, H-9a $\left.{ }^{\mathrm{NeuB}}, \mathrm{H}-8^{\mathrm{NeuA}}, \mathrm{H}-7^{\mathrm{NeuB}}\right), 3.85\left(\mathrm{~s}, 3 \mathrm{H}, \mathrm{CO}_{2} \mathrm{CH}_{3}{ }^{\mathrm{NeuA}}\right), 3.81\left(\mathrm{~s}, 3 \mathrm{H}, \mathrm{CO}_{2} \mathrm{CH}_{3}{ }^{\mathrm{NeuB}}\right)$, $3.75-3.68\left(\mathrm{~m}, 2 \mathrm{H}, \mathrm{H}-9 \mathrm{~b}^{\mathrm{NeuB}}, \mathrm{H}-5^{\mathrm{NeuB}}\right), 3.54\left(\mathrm{~s}, 3 \mathrm{H}, \mathrm{OCH}_{3}{ }^{\mathrm{NeuA}}\right), 3.34\left(\mathrm{~d}, J=10.6 \mathrm{~Hz}, 1 \mathrm{H}, \mathrm{H}-6^{\mathrm{NeuB}}\right), 2.88\left(\mathrm{dd}, J=12.7,4.5 \mathrm{~Hz}, 1 \mathrm{H}, \mathrm{H}-3 \mathrm{eq}^{\mathrm{NeuA}}\right), 2.51$ (dd, $J=12.5,4.4 \mathrm{~Hz}, 1 \mathrm{H}, \mathrm{H}-3 \mathrm{eq}^{\mathrm{NeuB}}$ ), 2.18 (s, 3H, Ac), 2.16-2.13 (m, 1H, H-3ax ${ }^{\mathrm{NeuA}}$ ), 2.09 (s, 3H, Ac), 2.09 (s, 3H, Ac), 2.05 (s, 3H, Ac), 2.01 (s, $3 \mathrm{H}, \mathrm{Ac}), 1.78$ (s, 3H, Ac), 1.50 (t, $\left.J=12.5,1 \mathrm{H}, \mathrm{H}-3 \mathrm{ax}^{\mathrm{NeuB}}\right) ;{ }^{13} \mathrm{C} \mathrm{NMR}\left(100 \mathrm{MHz}, \mathrm{CDCl}_{3}\right) \delta 171.5$ (C), $171.4(\mathrm{C}), 171.0(\mathrm{C}), 170.5(\mathrm{C}), 170.5(\mathrm{C})$, $170.4(\mathrm{C}), 169.9(\mathrm{C}), 168.2(\mathrm{C}), 167.6(\mathrm{C}), 166.6(\mathrm{C}), 166.4(\mathrm{C}), 166.3(\mathrm{C}), 138.3(\mathrm{C}), 133.7(\mathrm{CH}), 133.6(\mathrm{C}), 133.2(\mathrm{CH}), 133.2(\mathrm{CH}), 131.9(\mathrm{CH})$, $130.7(\mathrm{CH}), 130.0(\mathrm{C}), 129.9(\mathrm{CH}), 129.9(\mathrm{CH}), 129.2(\mathrm{C}), 128.8(\mathrm{CH}), 128.7(\mathrm{CH}), 128.5(\mathrm{CH}), 128.5(\mathrm{CH}), 128.4(\mathrm{CH}), 127.8(\mathrm{CH}), 127.7(\mathrm{CH})$, 127.6 (CH), $98.5(\mathrm{C}), 96.5(\mathrm{C}), 86.4(\mathrm{CH}), 76.3(\mathrm{CH}), 74.5(\mathrm{CH}), 73.8(\mathrm{CH}), 73.3\left(\mathrm{CH}_{2}\right), 73.0(\mathrm{CH}), 71.9(\mathrm{CH}), 70.1(\mathrm{CH}), 70.0(\mathrm{CH}), 69.3(\mathrm{CH})$, 68.6 (CH), $68.3(\mathrm{CH}), 68.3(\mathrm{CH}), 68.1(\mathrm{CH}), 66.2(\mathrm{CH}), 63.0\left(\mathrm{CH}_{2}\right), 62.7\left(\mathrm{CH}_{2}\right), 62.2\left(\mathrm{CH}_{2}\right), 58.7\left(\mathrm{CH}_{3}\right), 53.4\left(\mathrm{CH}_{3}\right), 53.3\left(\mathrm{CH}_{3}\right), 51.4(\mathrm{CH}), 49.2$ $(\mathrm{CH}), 37.9\left(\mathrm{CH}_{2}\right), 37.6\left(\mathrm{CH}_{2}\right), 23.3\left(\mathrm{CH}_{3}\right), 22.0\left(\mathrm{CH}_{3}\right), 21.0\left(\mathrm{CH}_{3}\right), 21.0(\mathrm{CH}), 20.9\left(\mathrm{CH}_{3}\right), 20.9\left(\mathrm{CH}_{3}\right)$; HRMS-ESI [M + Na] $]^{+} \mathrm{Calcd}$ for $\mathrm{C}_{75} \mathrm{H}_{84} \mathrm{~N}_{2} \mathrm{O}_{30} \mathrm{SNa}$ 1547.4722, Found 1547.4724 . 


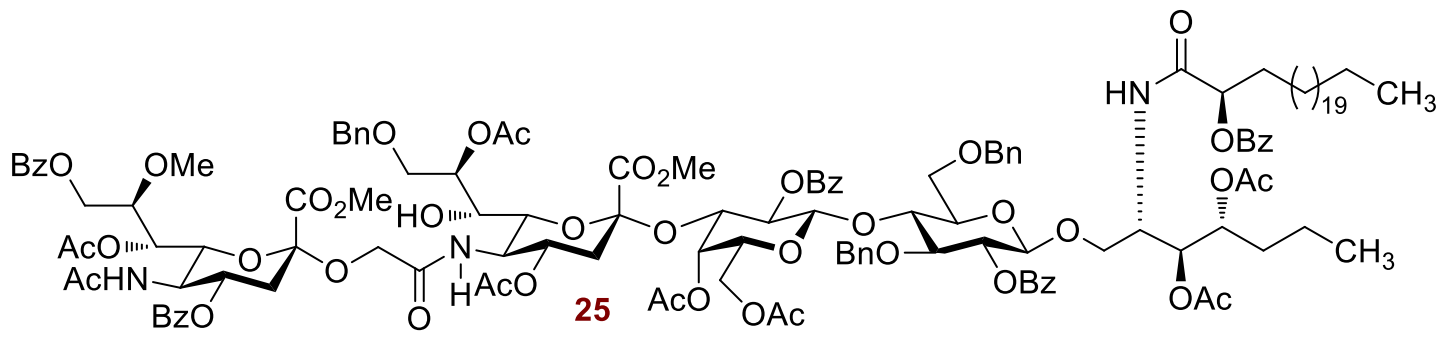

(Methyl 5-acetamido-7-O-acetyl-4,9-di- $O$-benzoyl-3,5-dideoxy-8-O-methyl-D-glycero- $\alpha$-D-galacto-2-nonulopyranosylonate)-(2 $\rightarrow 11$ )(methyl 4,8-di-O-acetyl-9-O-benzyl-3,5-dideoxy-5-glycolylamido-D-glycero- $\alpha$-D-galacto-2-nonulopyranosylonate)-(2 $\rightarrow 3$ )-2- $O$-benzoyl-4,6-

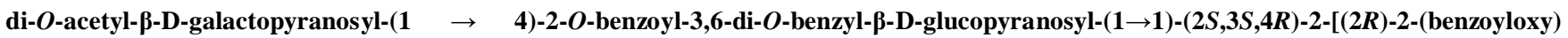
tetracosanoylamino]-3,4-di- $\boldsymbol{O}$-acetyloctadecan-1,3,4-triol (25) A mixture of the donor 24 (0.014 g, $8.94 \mu \mathrm{mol})$, acceptor 4 (0.014 g, $10.73 \mu \mathrm{mol})$, and activated $3 \AA$ powdered molecular sieves $(0.083 \mathrm{~g})$ in anhydrous $\mathrm{CH}_{2} \mathrm{Cl}_{2}(0.9 \mathrm{~mL})$ was stirred at room temperature for $1 \mathrm{~h}$ under nitrogen to remove any trace amounts of water. The reaction mixture was then cooled to $-30{ }^{\circ} \mathrm{C}$ followed by addition of $N$-iodosuccinimide $(0.004 \mathrm{~g}, 17.88$ $\mu \mathrm{mol})$ and $\mathrm{TfOH}(1 \mu \mathrm{L}, 8.94 \mu \mathrm{mol})$. Continuously stirred at this temperature for $2 \mathrm{~h}$, the reaction mixture was carefully quenched with triethylamine and then filtered through a short pad of Celite. The filtrate was washed with cold saturated aqueous $\mathrm{Na}_{2} \mathrm{~S}_{2} \mathrm{O}_{3}$, brine, dried over $\mathrm{MgSO}_{4}$, filtered, and concentrated in vacuo. The observed yellowish syrup was purified by flash column chromatography on silica gel using ethyl acetate and hexanes $(1: 1, \mathrm{v} / \mathrm{v})$ as the eluent to give $0.024 \mathrm{~g}$ of a white solid 25 in $83 \%$ yield: $R_{f}=0.38\left(\right.$ ethyl acetate: hexanes $=3: 2(\mathrm{v} / \mathrm{v})$ ); $[\alpha]^{28}{ }_{\mathrm{D}}$ +37.2 (c 0.11, $\mathrm{CHCl}_{3}$ ); FT-IR (neat) $v_{\max } 3337,3065,2926,1722,1658,1551,1440,1373,1280,1245,1205,1129,1083,1029,753,712 \mathrm{~cm}^{-1} ;{ }^{1} \mathrm{H}$ NMR $\left(400 \mathrm{MHz}, \mathrm{CDCl}_{3}\right) \delta 8.29(\mathrm{~d}, J=7.4 \mathrm{~Hz}, 2 \mathrm{H}, \mathrm{ArH}), 8.07(\mathrm{~d}, J=7.4 \mathrm{~Hz}, 2 \mathrm{H}, \mathrm{ArH}), 7.98(\mathrm{~d}, J=7.4 \mathrm{~Hz}, 2 \mathrm{H}, \operatorname{ArH}), 7.93(\mathrm{~d}, J=7.4 \mathrm{~Hz}, 2 \mathrm{H}$, $\operatorname{ArH}), 7.86(\mathrm{~d}, J=7.4 \mathrm{~Hz}, 2 \mathrm{H}, \mathrm{ArH}), 7.61-7.35(\mathrm{~m}, 14 \mathrm{H}, \mathrm{ArH}), 7.31-7.18(\mathrm{~m}, 11 \mathrm{H}, \mathrm{ArH}), 7.11-7.06(\mathrm{~m}, 5 \mathrm{H}, \mathrm{ArH}), 6.77\left(\mathrm{~d}, J=8.4 \mathrm{~Hz}, 2 \mathrm{H}, \mathrm{NH}^{\mathrm{NeuB}}\right.$, $\left.\mathrm{NH}^{\mathrm{Cer}}\right), 5.48-5.45\left(\mathrm{~m}, 1 \mathrm{H}, \mathrm{H}-8^{\mathrm{NeuB}}\right), 5.35\left(\mathrm{dd}, J=10.3,8.1 \mathrm{~Hz}, 1 \mathrm{H}, \mathrm{H}-2^{\mathrm{Gal}}\right), 5.29-5.24\left(\mathrm{~m}, 2 \mathrm{H}, \mathrm{H}-7^{\mathrm{NeuA}}, \mathrm{NH}^{\mathrm{NeuA}}\right), 5.22-5.15\left(\mathrm{~m}, 1 \mathrm{H}, \mathrm{H}-4^{\mathrm{NeuA}}\right), 5.08-$ $5.05\left(\mathrm{~m}, 2 \mathrm{H}, \mathrm{H}-2^{\mathrm{Gal}}, \mathrm{H}-2{ }^{\text {,Cer }}\right), 5.01-4.86\left(\mathrm{~m}, 7 \mathrm{H}, \mathrm{H}-4^{\mathrm{NeuB}}, \mathrm{H}-4^{\mathrm{Cer}}, \mathrm{H}-3^{\mathrm{Gal}}, \mathrm{H}-3^{\mathrm{Cer}}, \mathrm{H}-2^{\mathrm{Glc}}, \mathrm{H}-1^{\mathrm{Gal}}, \mathrm{CHaHbPh}\right), 4.83\left(\mathrm{~d}, J=4.7 \mathrm{~Hz}, 1 \mathrm{H}, \mathrm{OH}-7^{\mathrm{NeuB}}\right), 4.74$ $\left(\mathrm{dd}, J=12.2,2.6 \mathrm{~Hz}, 1 \mathrm{H}, \mathrm{H}_{-} \mathrm{a}^{\mathrm{NeuA}}\right.$ ), 4.54-4.47 (m, 2H, CHaHbPh), 4.43-4.35 (m, 3H, CHaHbPh, OCHaHbCO), 4.31-4.19 (m, 5H, H-6 ${ }^{\mathrm{NeuA}}, \mathrm{H}-$ $\left.5^{\mathrm{NeuA}}, \mathrm{H}-2^{\mathrm{Cer}}, \mathrm{H}-1^{\mathrm{Glc}}, \mathrm{CHaHbPh}\right), 4.10\left(\mathrm{dd}, \mathrm{J}=12.3,4.2 \mathrm{~Hz}, 1 \mathrm{H}, \mathrm{H}-9 \mathrm{~b}^{\mathrm{NeuA}}\right), 4.05-3.89$ (m, 5H, H-7 $\left.{ }^{\mathrm{NeuB}}, \mathrm{H}-6 \mathrm{a}^{\mathrm{Gal}}, \mathrm{H}-5^{\mathrm{Gal}}, \mathrm{H}-4^{\mathrm{Glc}}, \mathrm{OCHaHbCO}\right), 3.85$ (s, 3H, $\mathrm{CO}_{2} \mathrm{CH}_{3}{ }^{\mathrm{NeuA}}$ ), 3.83-3.67 (m, 5H, H-9 $\left.{ }^{\mathrm{NeuB}}, \mathrm{H}-8^{\mathrm{NeuA}}, \mathrm{H}-6 \mathrm{~b}^{\mathrm{Gal}}, \mathrm{H}-5^{\mathrm{NeuB}}, \mathrm{H}-1 \mathrm{a}^{\mathrm{Cer}}\right), 3.81$ (s, 3H, $\mathrm{CO}_{2} \mathrm{CH}_{3}{ }^{\mathrm{NeuB}}$ ), 3.59-3.47 (m, 3H, H-9b ${ }^{\mathrm{NeuB}}, \mathrm{H}-$ $\left.3^{\mathrm{Glc}}, \mathrm{H}-1 \mathrm{~b}^{\mathrm{Cer}}\right), 3.54\left(\mathrm{~s}, 3 \mathrm{H}, \mathrm{OCH}_{3}{ }^{\mathrm{NeuA}}\right), 3.40\left(\mathrm{dd}, J=10.9,3.7 \mathrm{~Hz}, 1 \mathrm{H}, \mathrm{H}-6 \mathrm{a}^{\mathrm{Glc}}\right), 3.33\left(\mathrm{~d}, J=10.0 \mathrm{~Hz}, 1 \mathrm{H}, \mathrm{H}-6^{\mathrm{NeuB}}\right), 3.25(\mathrm{~d}, J=10.2 \mathrm{~Hz}, 1 \mathrm{H}, \mathrm{H}-$ $6 \mathrm{~b}^{\mathrm{Glc}}$ ), $3.03\left(\mathrm{~d}, J=9.2 \mathrm{~Hz}, 1 \mathrm{H}, \mathrm{H}-5^{\mathrm{Glc}}\right), 2.89\left(\mathrm{dd}, J=12.8,4.6 \mathrm{~Hz}, 1 \mathrm{H}, \mathrm{H}-3 \mathrm{eq}^{\mathrm{NeuA}}\right.$ ), $2.51\left(\mathrm{dd}, J=12.2,4.3 \mathrm{~Hz}, 1 \mathrm{H}, \mathrm{H}-3 \mathrm{eq}{ }^{\mathrm{NeuB}}\right), 2.16(\mathrm{t}, J=12.3 \mathrm{~Hz}$, $1 \mathrm{H}, \mathrm{H}-3 \mathrm{ax}^{\mathrm{NeuA}}$ ), 2.13 (s, 3H, Ac), 2.09 (s, 3H, Ac), 2.06 (s, 3H, Ac), 2.01 (s, 3H, Ac), 1.93 (s, 3H, Ac), 1.90 (s, 3H, Ac), 1.84 (s, 3H, Ac), 1.78 (s, $3 \mathrm{H}, \mathrm{Ac}$ ), 1.76-1.74 (m, 2H, H-3 ${ }^{\mathrm{Cer}}$ ), 1.62-1.47 (m, 2H, H-5a ${ }^{\mathrm{Cer}}, \mathrm{H}-5 \mathrm{~b}^{\mathrm{Cer}}$ ), 1.48 (t, $J=12.2 \mathrm{~Hz}, 1 \mathrm{H}, \mathrm{H}-3 \mathrm{ax}^{\mathrm{NeuB}}$ ), 1.25-1.19 (m, $65 \mathrm{H}$, aliphatic), 0.87 $\left(\mathrm{t}, J=6.7 \mathrm{~Hz}, 6 \mathrm{H}, \mathrm{CH}_{3}\right) ;{ }^{13} \mathrm{C}$ NMR $\left(100 \mathrm{MHz}, \mathrm{CDCl}_{3}\right) \delta 171.5$ (C), 170.8 (C), 170.5 (C), 170.4 (C), 170.3 (C), 170.1 (C), 169.9 (C), 169.8 (C), $169.8(\mathrm{C}), 168.0(\mathrm{C}), 167.6(\mathrm{C}), 166.6(\mathrm{C}), 166.4(\mathrm{C}), 166.2(\mathrm{C}), 166.2(\mathrm{C}), 165.2(\mathrm{C}), 165.1(\mathrm{C}), 138.7(\mathrm{C}), 138.2(\mathrm{C}), 138.2(\mathrm{C}), 133.7(\mathrm{CH})$, $133.4(\mathrm{CH}), 133.3(\mathrm{CH}), 133.1(\mathrm{CH}), 130.7(\mathrm{CH}), 129.9(\mathrm{CH}), 129.5(\mathrm{C}), 129.2(\mathrm{C}), 128.7(\mathrm{CH}), 128.5(\mathrm{CH}), 128.4(\mathrm{CH}), 128.4(\mathrm{CH}), 128.3(\mathrm{CH})$, 128.0 (CH), $127.8(\mathrm{CH}), 127.7(\mathrm{CH}), 127.7(\mathrm{CH}), 127.6(\mathrm{CH}), 127.2(\mathrm{CH}), 100.9(\mathrm{CH}), 99.8(\mathrm{CH}), 98.5(\mathrm{C}), 96.6(\mathrm{C}), 80.4(\mathrm{CH}), 76.7(\mathrm{CH}), 76.4$ $(\mathrm{CH}), 74.7(\mathrm{CH}), 74.6(\mathrm{CH}), 74.4\left(\mathrm{CH}_{2}\right), 73.9(\mathrm{CH}), 73.3\left(\mathrm{CH}_{2}\right), 73.3(\mathrm{CH}), 73.0\left(\mathrm{CH}_{2}\right), 73.0(\mathrm{CH}), 72.7(\mathrm{CH}), 72.1(\mathrm{CH}), 70.9(\mathrm{CH}), 70.8(\mathrm{CH})$, $70.7(\mathrm{CH}), 70.1(\mathrm{CH}), 70.0(\mathrm{CH}), 69.1\left(\mathrm{CH}_{2}\right), 68.3(\mathrm{CH}), 68.2(\mathrm{CH}), 68.0(\mathrm{CH}), 67.7\left(\mathrm{CH}_{2}\right), 66.0(\mathrm{CH}), 64.9\left(\mathrm{CH}_{2}\right), 63.0\left(\mathrm{CH}_{2}\right), 62.2\left(\mathrm{CH}_{2}\right), 61.6$ $\left(\mathrm{CH}_{2}\right), 58.7\left(\mathrm{CH}_{3}\right), 53.4\left(\mathrm{CH}_{3}\right), 51.5(\mathrm{CH}), 49.3(\mathrm{CH}), 47.7(\mathrm{CH}), 37.9\left(\mathrm{CH}_{2}\right), 37.6\left(\mathrm{CH}_{2}\right), 21.1\left(\mathrm{CH}_{2}\right), 31.8\left(\mathrm{CH}_{2}\right), 29.8\left(\mathrm{CH}_{2}\right), 29.8\left(\mathrm{CH}_{2}\right), 29.7$ $\left(\mathrm{CH}_{2}\right), 29.7\left(\mathrm{CH}_{2}\right), 29.6\left(\mathrm{CH}_{2}\right), 29.5\left(\mathrm{CH}_{2}\right), 29.4\left(\mathrm{CH}_{2}\right), 28.5\left(\mathrm{CH}_{2}\right), 25.7\left(\mathrm{CH}_{2}\right), 24.9\left(\mathrm{CH}_{2}\right), 23.3\left(\mathrm{CH}_{3}\right), 22.8\left(\mathrm{CH}_{2}\right), 21.8\left(\mathrm{CH}_{3}\right), 21.1\left(\mathrm{CH}_{3}\right), 21.0$ $\left(\mathrm{CH}_{3}\right), 20.9\left(\mathrm{CH}_{3}\right), 20.7\left(\mathrm{CH}_{3}\right), 20.7\left(\mathrm{CH}_{3}\right), 14.3\left(\mathrm{CH}_{3}\right)$; HRMS-ESI [M + Na] ${ }^{+} \mathrm{Calcd}$ for $\mathrm{C}_{149} \mathrm{H}_{197} \mathrm{~N}_{3} \mathrm{O}_{44} \mathrm{Na} 2756.3196$, Found 2756.3310.

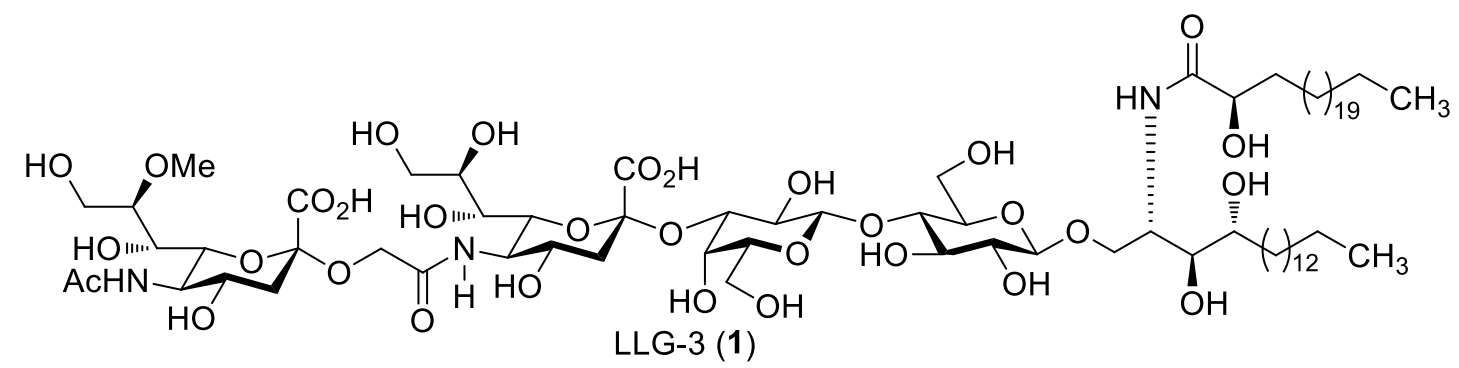

Synthesis of Target Compound LLG-3 (1) The ester $25(0.020 \mathrm{~g}, 7.20 \mu \mathrm{mol})$ and $\mathrm{LiCl}(0.012 \mathrm{~g}, 0.29 \mathrm{mmol})$ was dissolved in pyridine $(0.7 \mathrm{~mL})$ at room temperature and then the mixture was continuously stirred for $11 \mathrm{~h}$ at $120{ }^{\circ} \mathrm{C}$. The reaction mixture was extracted with ethyl acetate, washed with $1 \mathrm{~N} \mathrm{HCl}$, dried over $\mathrm{MgSO}_{4}$, and concentrated in vacuo. The resulting compound $\left(R_{f}=0.43\left(\mathrm{MeOH} \mathrm{CH}_{2} \mathrm{Cl} l_{2}=1: 5(\mathrm{v} / \mathrm{v})\right)\right.$ and 
$\mathrm{Pd}(\mathrm{OH})_{2} / \mathrm{C}(0.033 \mathrm{~g})$ was dissolved in $\mathrm{MeOH}(0.7 \mathrm{~mL})$ at room temperature. The reaction mixture was stirred under hydrogen balloon at room temperature for $4 \mathrm{~h}$. The $\mathrm{Pd}(\mathrm{OH})_{2} / \mathrm{C}$ was removed through a short pad of $\mathrm{SiO}_{2} / \mathrm{Celite}$ and the filter was washed with $\mathrm{MeOH}$. The filtrate was concentrated in vacuo. The resulting compound $\left(R_{f}=0.08\left(\mathrm{MeOH}: \mathrm{CH}_{2} \mathrm{Cl}_{2}=1: 5(\mathrm{v} / \mathrm{v})\right)\right.$ was dissolved in $\mathrm{MeOH}(0.7 \mathrm{~mL})$ and then $\mathrm{MeONa}(4 \mathrm{mg}$, $72.04 \mu \mathrm{mol})$ was added to this solution at $0{ }^{\circ} \mathrm{C}$. After stirring for $6 \mathrm{~h}$ at $50{ }^{\circ} \mathrm{C}$, the solution was neutralized with Dowex $50 \mathrm{w} \mathrm{X} 8\left[\mathrm{H}^{+}\right]$. The resin was filtered out and washed with $\mathrm{MeOH} / \mathrm{CHCl}_{3} / \mathrm{H}_{2} \mathrm{O}(4 / 6 / 1, \mathrm{v} / \mathrm{v} / \mathrm{v})$. The filtrate was concentrated under reduced pressure to give a white solid residue. The residue were purified by flash column chromatography on silica gel using $\mathrm{MeOH} / \mathrm{CHCl}_{3} / \mathrm{H}_{2} \mathrm{O}(4 / 6 / 1$, v/v/v) as the eluent to give to produce $0.008 \mathrm{~g}$ of a white solid LLG-3 in $73 \%$ yield. $R_{f}=0.30\left(\mathrm{MeOH} / \mathrm{CHCl}_{3} / \mathrm{H}_{2} \mathrm{O}(4 / 6 / 1, \mathrm{v} / \mathrm{v} / \mathrm{v})\right) ;[\alpha]^{22}{ }_{\mathrm{D}}+16.9\left(\mathrm{c} 0.24, \mathrm{CHCl} / \mathrm{MeOH} / \mathrm{H}_{2} \mathrm{O}=\right.$ 1/2/0.2); FT-IR (neat) $v_{\max } 3418,2957,2921,2851,1740,1652,1373,1268,1232,1102,1074,1039 \mathrm{~cm}^{-1} ;{ }^{1} \mathrm{H} \mathrm{NMR}\left(700 \mathrm{MHz}, \mathrm{CD}_{3} \mathrm{OD}\right) \mathrm{d} 4.43$ (d, $J=7.8 \mathrm{~Hz}, 1 \mathrm{H}), 4.32(\mathrm{~d}, J=7.8 \mathrm{~Hz}, 1 \mathrm{H}), 4.31-4.22(\mathrm{~m}, 3 \mathrm{H}), 4.09-4.01(\mathrm{~m}, 4 \mathrm{H}), 3.93-3.76(\mathrm{~m}, 13 \mathrm{H}), 3.67-3.63(\mathrm{~m}, 4 \mathrm{H}), 3.59-3.50(\mathrm{~m}, 7 \mathrm{H}), 3.49(\mathrm{~s}$, $3 \mathrm{H}), 3.44-3.40(\mathrm{~m}, 2 \mathrm{H}), 3.25(\mathrm{t}, J=8.4 \mathrm{~Hz}, 1 \mathrm{H}), 2.87(\mathrm{dd}, J=12.4,3.3 \mathrm{~Hz}, 1 \mathrm{H}), 2.65(\mathrm{~d}, J=10.2 \mathrm{~Hz}, 1 \mathrm{H}), 1.99(\mathrm{~s}, 3 \mathrm{H}), 1.76-1.54(\mathrm{~m}, 6 \mathrm{H}), 1.43-$ 1.29 (m, 64H), 0.90 (t, J=7.0 Hz, 6H); ${ }^{13} \mathrm{C}$ NMR (175 MHz, CD $\left.{ }_{3} \mathrm{OD}\right)$ d $177.1(\mathrm{C}), 175.2(\mathrm{C}), 174.9(\mathrm{C}), 105.1(\mathrm{CH}), 104.5$ (CH), $101.9(\mathrm{C}), 101.1$ (C), $81.7(\mathrm{CH}), 80.7(\mathrm{CH}), 77.7(\mathrm{CH}), 77.1(\mathrm{CH}), 76.5(\mathrm{CH}), 76.2(\mathrm{CH}), 75.3(\mathrm{CH}), 74.6(\mathrm{CH}), 74.5(\mathrm{CH}), 74.5(\mathrm{CH}), 73.1(\mathrm{CH}), 73.0(\mathrm{CH}), 72.9$ $(\mathrm{CH}), 70.9(\mathrm{CH}), 69.9(\mathrm{CH}), 69.9\left(\mathrm{CH}_{2}\right), 69.5(\mathrm{CH}), 69.5(\mathrm{CH}), 69.0(\mathrm{CH}), 64.7\left(\mathrm{CH}_{2}\right), 64.7\left(\mathrm{CH}_{2}\right), 62.7\left(\mathrm{CH}_{2}\right), 61.9\left(\mathrm{CH}_{2}\right), 61.7\left(\mathrm{CH}_{2}\right), 59.0\left(\mathrm{CH}_{3}\right)$, $54.1(\mathrm{CH}), 54.0(\mathrm{CH}), 41.9\left(\mathrm{CH}_{2}\right), 41.6\left(\mathrm{CH}_{2}\right), 35.7\left(\mathrm{CH}_{2}\right), 33.1\left(\mathrm{CH}_{2}\right), 33.1\left(\mathrm{CH}_{2}\right), 32.8\left(\mathrm{CH}_{2}\right), 31.0\left(\mathrm{CH}_{2}\right), 30.9\left(\mathrm{CH}_{2}\right), 30.8\left(\mathrm{CH}_{2}\right), 30.8\left(\mathrm{CH}_{2}\right)$, $30.5\left(\mathrm{CH}_{2}\right), 30.5\left(\mathrm{CH}_{2}\right), 27.1\left(\mathrm{CH}_{2}\right), 26.1\left(\mathrm{CH}_{2}\right), 23.8\left(\mathrm{CH}_{2}\right), 23.8\left(\mathrm{CH}_{2}\right), 22.7\left(\mathrm{CH}_{3}\right), 14.5\left(\mathrm{CH}_{3}\right), 14.5\left(\mathrm{CH}_{3}\right)$; HRMS-ESI [M/2 - H] Calcd for $\mathrm{C}_{77} \mathrm{H}_{139} \mathrm{~N}_{3} \mathrm{O}_{32} 808.9671$, Found 808.9673.

\section{Materials and Methods for Biological Studies:}

\section{(A) Results:}

\section{LLG3 promotes neurite outgrowth in primary cortical neurons}

We investigated the effect of LLG3 on neurite outgrowth in primary cortical neurons, and compared with other gangliosides such as GM1 and DSG-A. The cultured cortical neurons were treated with or without $35 \mu \mathrm{M}$ of LLG-3, GM1, DSG-A for $24 \mathrm{~h}$, respectively, and then subjected to immunofluorescence staining using anti-MAP2 antibody. As shown in Fig.1, LLG-3, but not GM1 or DSG-A, significantly increased the average total neurite length per cell by 1.45 -fold of control group.

\section{(B) Methods:}

\section{Primary cortical neuron culture}

All experimental procedures were approved by the Institutional Animal Care and Use Committee at the National Defense Medical Center (Taipei, Taiwan). Rat primary cortical neuron culture was performed according to a published procedure ${ }^{[1]}$. One-day-old Sprague-Dawley rats were sacrificed, their brains were collected, and the meninges and blood vessels were carefully removed. The cerebral cortex was placed in icecold calcium and magnesium-free Hank's balanced salt solution (HBSS; Invitrogen, Carlsbad, CA), minced, digested with $0.125 \%$ trypsin and $0.05 \%$ DNase for $10 \mathrm{~min}$ at $37{ }^{\circ} \mathrm{C}$, resuspended in DMEM with 10\% FBS to inactivate the trypsin, and pelleted by centrifugation at 1,000 rpm for $10 \mathrm{~min}$. After resuspending the cell pellet in culture medium and adjusting the cell density to $1 \times 10^{6} \mathrm{cells} / \mathrm{ml}$, the neurons were seeded on coverslips or in culture dishes, which were precoated with poly-D-lysine, and cultured in medium comprising a 1:1 mixture of DMEM/F-12 and neurobasal medium (Invitrogen) supplemented with 5\% FBS, $0.5 \mathrm{mM} \mathrm{L}$-glutamine, 1\% penicillin-streptomycin and 2\% B-27 serum-free supplement (Invitrogen). On the third day after plating, nonneuronal cell proliferation was suppressed by treatment with $5 \mu \mathrm{M}$ cytosine-D-arabinofuranoside for $24 \mathrm{~h}$. On the 5-7 days of culture, the cells were used for experiments. Neurons were identified by positive staining with a neuron-specific antiMAP2 antibody (Sigma Chemical, St. Louis, MO); the percentage of MAP2-immunostained neurons was over 90\%.

\section{Neurite Outgrowth Assay}

Neurite outgrowth visible in MAP2-positive neuron images was quantified as the total length of neurites radiated from a single neuron. Images were acquired from randomly selected fields ( $\mathrm{n}=8 \sim 12)$ under fluorescence Microscope. The length of the total neurite of 40-50 neurons per condition was determined using NeuronJ/ImageJ software (NIH, Bethesda, MD). NeuronJ is an ImageJ plugin to facilitate the tracing and quantification of elongated and network image structures, in particular neurites in fluorescence microscopy images ${ }^{[2]}$. NeuroJ allows the user to interactively analyze running averages and statistic of neurite length, soma number, neurite attachment points, and neurite ending points from a single image. Each experimental condition was done in duplicate wells, and at least three independent experiments were conducted to acquire the final results. Approximately a total of 200-300 cells were counted in each group. 


\section{Statistics}

Quantitative data are presented as the mean \pm SD of at least three independent experiments. Statistical analysis of data was performed using an unpaired two-tailed $\mathrm{t}$ t-test or one-way analysis of variance followed by the Bonferroni post hoc test to determine statistical significance. Differences were considered to be significant at a $\mathrm{P}$ value $<0.05$; a $\mathrm{P}$ value $<0.01$ was considered highly statistically significant.
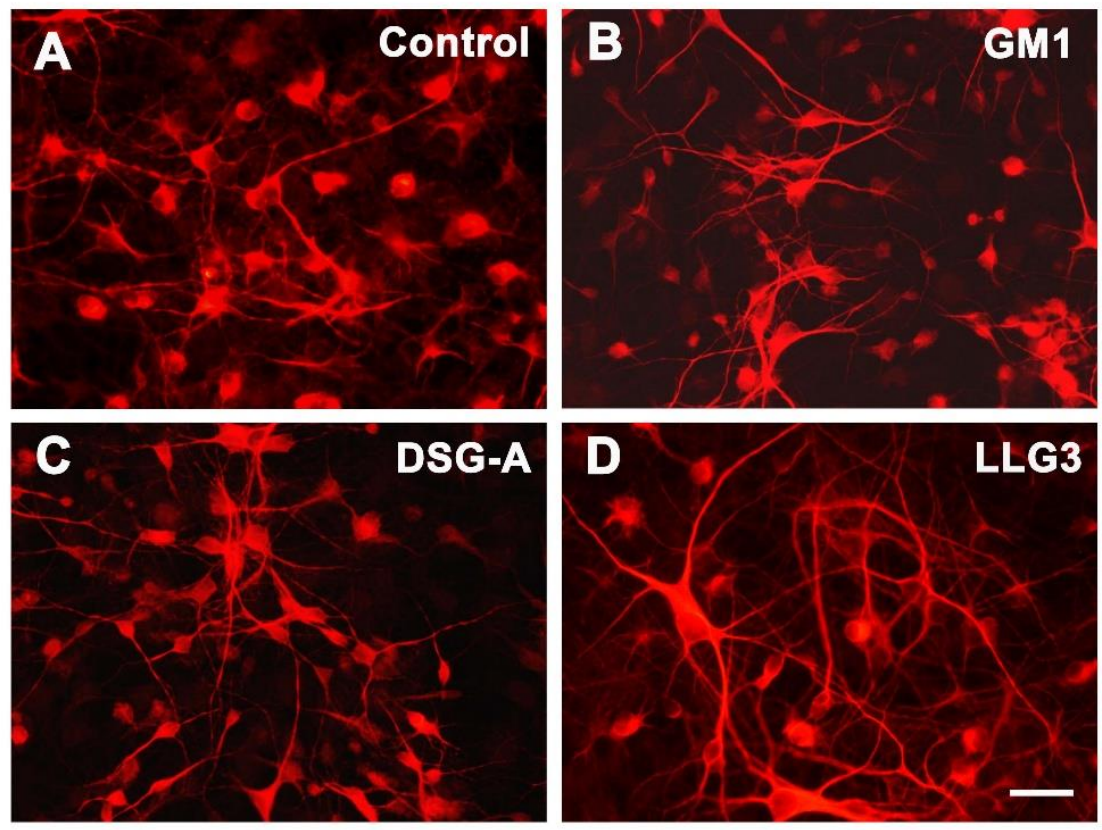

$\mathbf{E}$

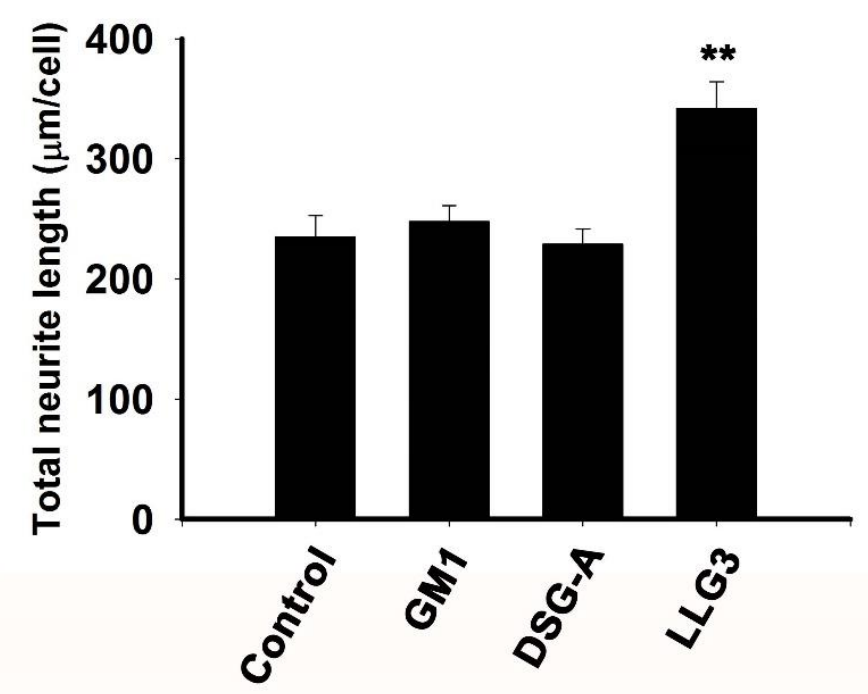

Figure 1. Effect of ganglioside GM1, DSG-A and LLG-3 on neurite outgrowth in primary cortical neurons. (A-D) Cultured cortical neurons were treated with or without $35 \mu \mathrm{M}$ of GM1, DSG-A and LLG-3 for $24 \mathrm{~h}$, respectively, and then subjected to immunofluorescence staining with anti-MAP2 antibody. (E) Quantitation of neurite outgrowth. The total neurite length are shown as mean \pm SD., $\mathrm{n}=3$ experiments with $200-300$ cells per experiment, $* * \mathrm{P}<0.01$ vs control. 


\section{References}

[1] Li, I.-H.; Ma, K.-H.; Weng, S.-J.; Huang, S.-S.; Liang, C.-M.; Huang, Y.-S. Autophagy Activation is Involved in 3,4Methylenedioxymethamphetamine ('Ecstasy')_-Induced Neurotoxicity in Cultured Cortical Neurons. PLoS One 2014, 9, e116565.

[2] Popko, J.; Fernandes, A.; Brites, D.; Lanier, L. M. Automated Analysis of NeuronJ Tracing Data. Cytometry. Part A 2009, 75, $371-376$. 


\section{Spectra of Compounds:}

\section{${ }^{1} \mathrm{H}$ NMR $\left(400 \mathrm{MHz}, \mathrm{CDCl}_{3}\right)$ of $\mathbf{6}$ :}

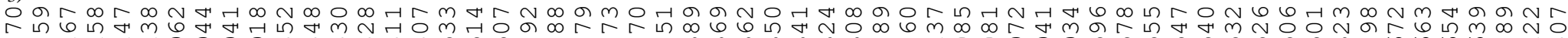
$\infty \omega_{0}^{0} 00000$

Current Data Parameters

NAME 70 Neu-5NAC-4,90Bz-

EXPNO ${ }^{7,8 \mathrm{Pi} \mathrm{C} \mathrm{O}-\mathrm{OCH} 2 \mathrm{CH} 2 \mathrm{OB}}$

EXPNO
PROCNO

F2 - Acquisition Parameters

Date_ 20180208

Time ${ }^{-}$

21.34

PROBHD $5 \mathrm{~mm}$ BBO $\mathrm{BB}-1 \mathrm{H}$

PULPROG

$\mathrm{BB}-13$
$\mathrm{zg} 30$
32768
$\mathrm{CDC} 13$

SOLVENT

NS

DS

SWH
FIDRES

FIDR
AQ
RG

$\mathrm{DW}$
$\mathrm{DE}$
$\mathrm{TE}$
$\mathrm{D}$

TE
D1
TD0

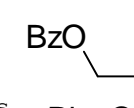

OPico

$\mathrm{CO}_{2} \mathrm{Me}$ $\mathrm{AcHN} \longrightarrow \mathrm{O}$

BzO

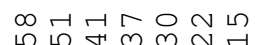

뉴맘

$6 \in \infty$

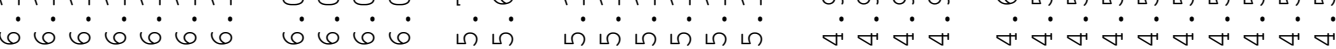
11111111110

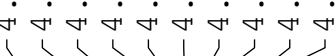

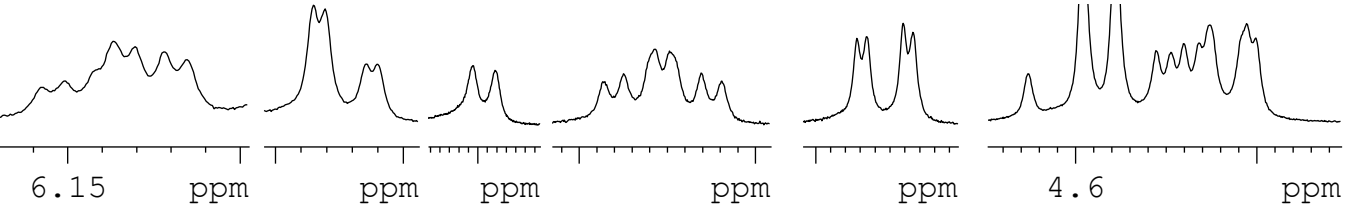
$6009.615 \mathrm{~Hz}$
$0.183399 \mathrm{~Hz}$ $2.7263477 \mathrm{sec}$

83.200 usec 6.50 usec $1.50000000 \mathrm{sec}$ $\begin{array}{lr}=======\text { CHANNEL } \mathrm{f} 1 \mathrm{1}======= \\ \text { NUC1 } & 1 \mathrm{H} \\ \text { P1 } & 14.00 \mathrm{usec} \\ \text { PL1 } & -1.00 \mathrm{~dB} \\ \text { PL1W } & 7.55784369 \mathrm{~W} \\ \text { SFO1 } & 400.1326010 \mathrm{MHz}\end{array}$ $400.1326010 \mathrm{MH}$ F2 - Processing parameters $\begin{array}{ll}\text { SI } & 32768 \\ \text { SF } & 400.1300095 \mathrm{MHz}\end{array}$ WSW $\quad 0$ $\mathrm{LB} \quad \mathrm{Hz}$ PC $\quad 1.00$<smiles>CCC</smiles>

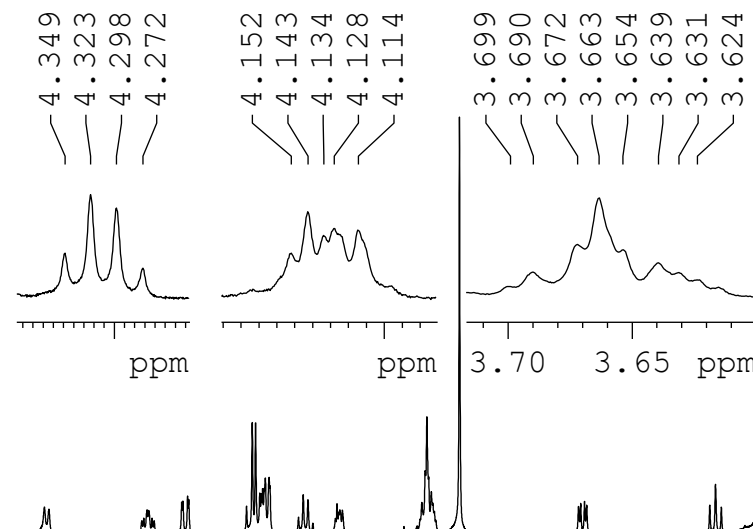

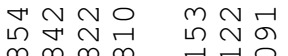
$\therefore \dot{\sim} \dot{\sim} \dot{ }$ Nij MM Nu 10

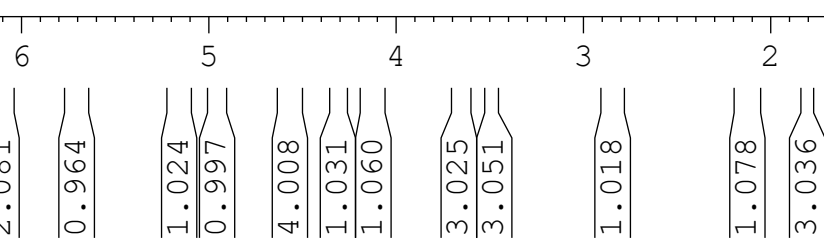


${ }^{13} \mathrm{C}$, DEPT-90 and DEPT-135 NMR (101 MHz, $\left.\mathrm{CDCl}_{3}\right)$ of $\mathbf{6}$ :

Bzico $\mathrm{CO}_{2} \mathrm{Me}$

PicoOn...

$\mathrm{AcHN} \underset{\mathrm{BzO}}{6} 6$
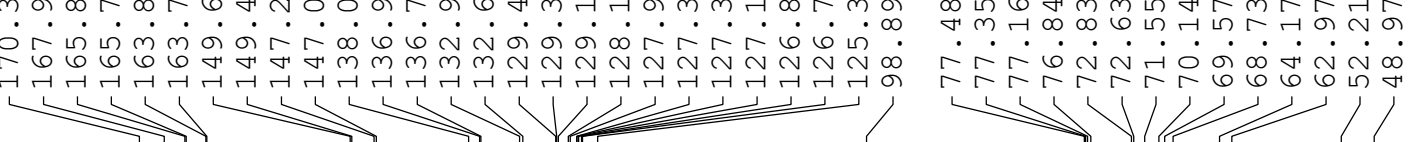

Current Data Parameters
NAME
70 Neu-5NAC-4, $90 \mathrm{Bz}-$

NAME 7, 8 PiCO-OCH2CH2OBn

EXPNO
PROCNO

F2 - Acquisition Parameters

Time 10.08

INSTRUM
PROBHD

PULPROG $5 \mathrm{~mm} \mathrm{BBO} \mathrm{BB-1H}$
zgpg30
65536

SD

NS

DS

$\begin{array}{lr}249 \\ \text { SWH } & 0 \\ \text { DSH } & 20161.291\end{array}$

$\begin{array}{ll}20161.291 \mathrm{~Hz} \\ \text { FIDRES } & 0.307637 \mathrm{~Hz}\end{array}$

RG

$\begin{array}{lr}\text { DW } & 24.800 \text { usec } \\ \text { DE } & 6.50 \text { usec }\end{array}$

$\begin{aligned} & 6.50 \text { usec } \\ & 295.5 \mathrm{~K}\end{aligned}$

$1.50000000 \mathrm{sec}$

D11 $\quad 0.03000000 \mathrm{sec}$

$=======$ CHANNEL f1 $========$
NUC1 $13 \mathrm{C}$
PC

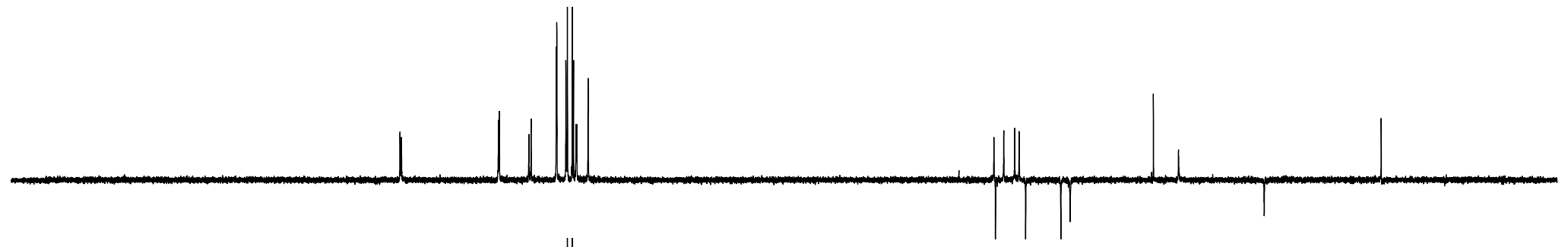

12.40 use

$\begin{array}{lrl}\text { PL1 } & \text { dB } \\ \text { PL1W } & 31.64976883 \mathrm{~W} \\ \text { SFO1 } & 100.6228303 \mathrm{MHz}\end{array}$

$=======$ CHANNEL f2 $========$

CPDPRG2
waltz16

$\begin{array}{lr}\text { NUC2 } & 11 \mathrm{H} \\ \text { PCPD2 } & 90.00 \mathrm{usec} \\ \text { PL2 } & -1.00 \mathrm{~dB} \\ \text { PL12 } & 15.16 \mathrm{~dB} \\ \text { PL13 } & 17.40 \mathrm{~dB} \\ \text { PL2W } & 7.55784369 \mathrm{~W} \\ \text { PL12W } & 0.18297760 \mathrm{~W} \\ \text { PL13W } & 0.10924409 \mathrm{~W} \\ \text { SF22 } & 400.1320007\end{array}$

F2 - Processing parameters

$\begin{array}{ll}\text { SI } & 32768 \\ \mathrm{SF} & 100.6127975 \mathrm{MHz}\end{array}$

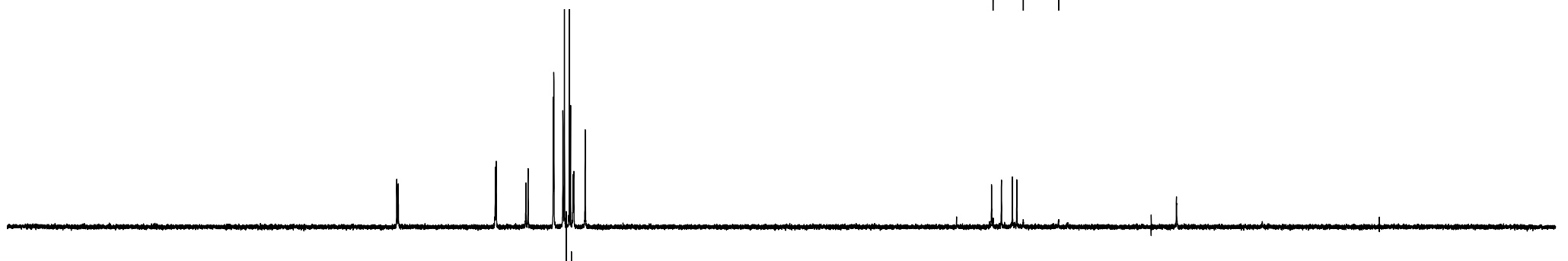
${ }_{0}^{0} \mathrm{~Hz}$ 
${ }^{1} \mathrm{H}$ NMR $\left(400 \mathrm{MHz}, \mathrm{CDCl}_{3}\right)$ of 7:

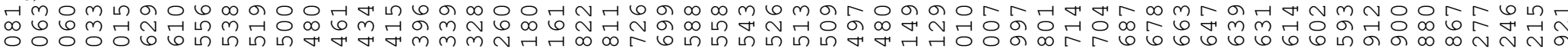

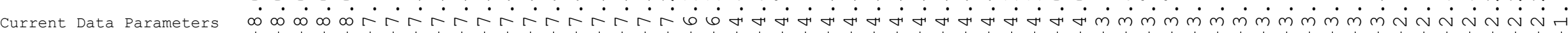

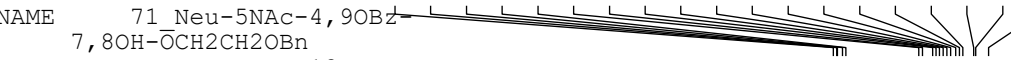
EXPNO ${ }^{7}$, $8 \mathrm{OH}-\mathrm{OCH} 2 \mathrm{CH} 2 \mathrm{OBn}$

F2 - Acquisition Parameters

$\begin{array}{lr}\text { Date } & 20171109 \\ \text { Time } & 12.29\end{array}$

$\begin{array}{lr}\text { Time } & 12.29 \\ \text { INSTRUM } & \text { spect } \\ \text { RROBD } & \text { BB }-1 \mathrm{H}\end{array}$

PROBHD

TD

DS

23

FIDRES $\quad 6009.615 \mathrm{~Hz}$

AQ $\quad 2.7263477 \mathrm{~Hz}$

$\begin{array}{lr}\text { RG } & 256 \\ \text { DW } & 83.200 \text { usec }\end{array}$

$\begin{array}{lr}\text { DE } & 6.50 \text { usec } \\ \text { TE } & 1.5000000 \mathrm{~K}\end{array}$

D1 $1.50000000 \mathrm{sec}$

TDO

$=======$ CHANNEL $\mathrm{fl}=======$

$\begin{array}{ll}\text { NUC1 } & 1 \mathrm{H} \\ \text { P1 } & 14.00 \text { usec } \\ \text { PL1 } & -1.00 \mathrm{~dB}\end{array}$

$\begin{array}{ll}\text { PL1W } & 7.55784369 \\ \text { PL1W } & 400.1326010 \mathrm{WHz}\end{array}$

SFO1 $\quad 400.1326010 \mathrm{MHz}$

F2 - Processing parameters

$\begin{array}{lr}\text { SI } & 32768 \\ \text { SF } & 400.1300097 \mathrm{MH}\end{array}$

WDW $\quad$ EM

$\begin{array}{lll}\text { SSB } & 0 \\ \text { LB } & 0 & \mathrm{~Hz} \\ \text { GB } & 0\end{array}$

$\mathrm{BzO}$

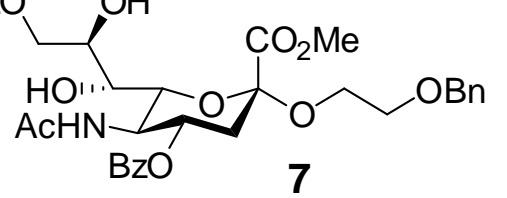

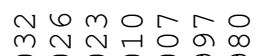

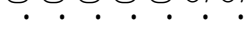
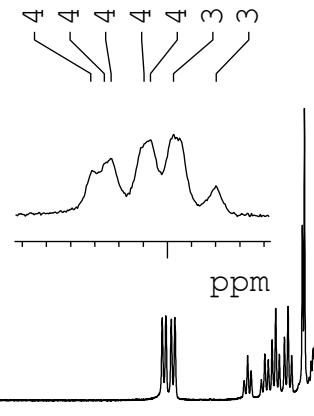

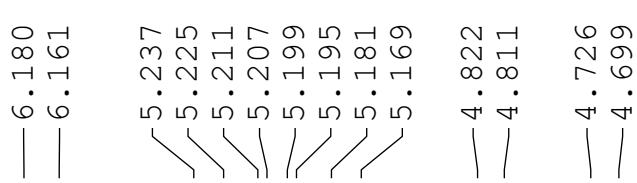

$\infty \infty m \in m a r \circ \quad N N N O G$

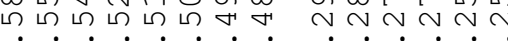

अवのmm

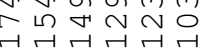

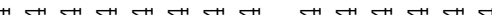
111

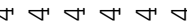

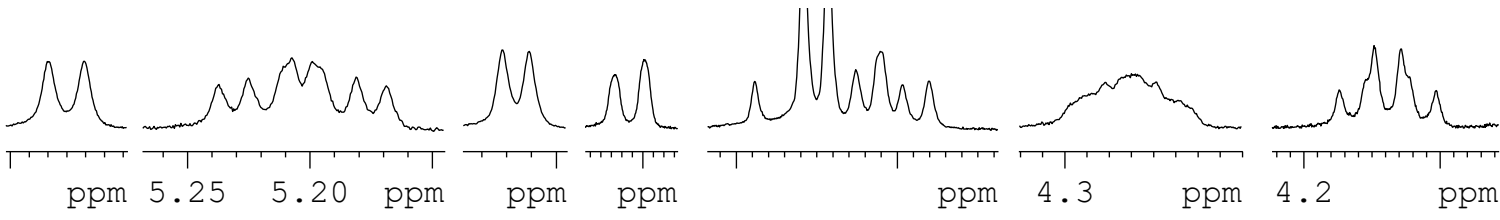

ppm $5.25 \quad 5.20 \quad \mathrm{ppm} \quad \mathrm{ppm} \quad \mathrm{ppm}$

pm 4.2

ppm

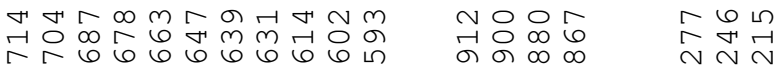

$\dot{m} \dot{m} \dot{m} \dot{m} \dot{m} \dot{m} \dot{m} \dot{m} \quad \dot{\sim} \dot{\sim} \dot{\sim} \quad \dot{\sim} \dot{\sim}$

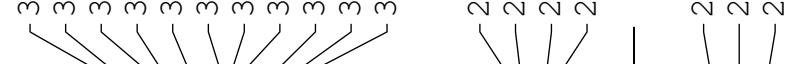

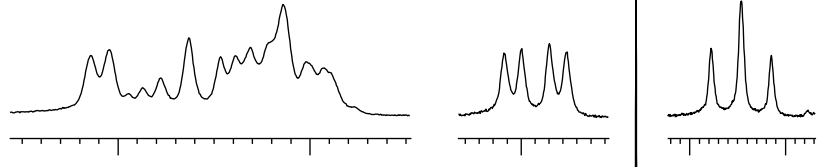

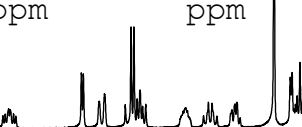

W

ppm
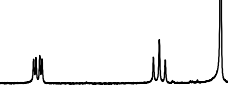

10

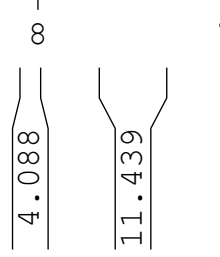

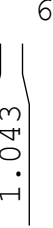

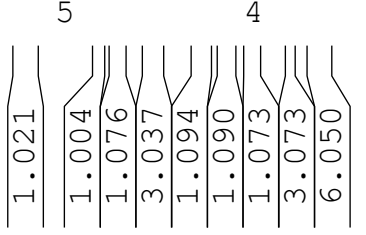

$\left|\begin{array}{r}3 \\ 0 \\ 0 \\ 0 \\ 0 \\ -1\end{array}\right|$

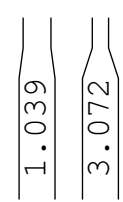

0 ppm 
${ }^{13} \mathrm{C}$, DEPT-90 and DEPT-135 NMR (101 MHz, $\mathrm{CDCl}_{3}$ ) of 7:

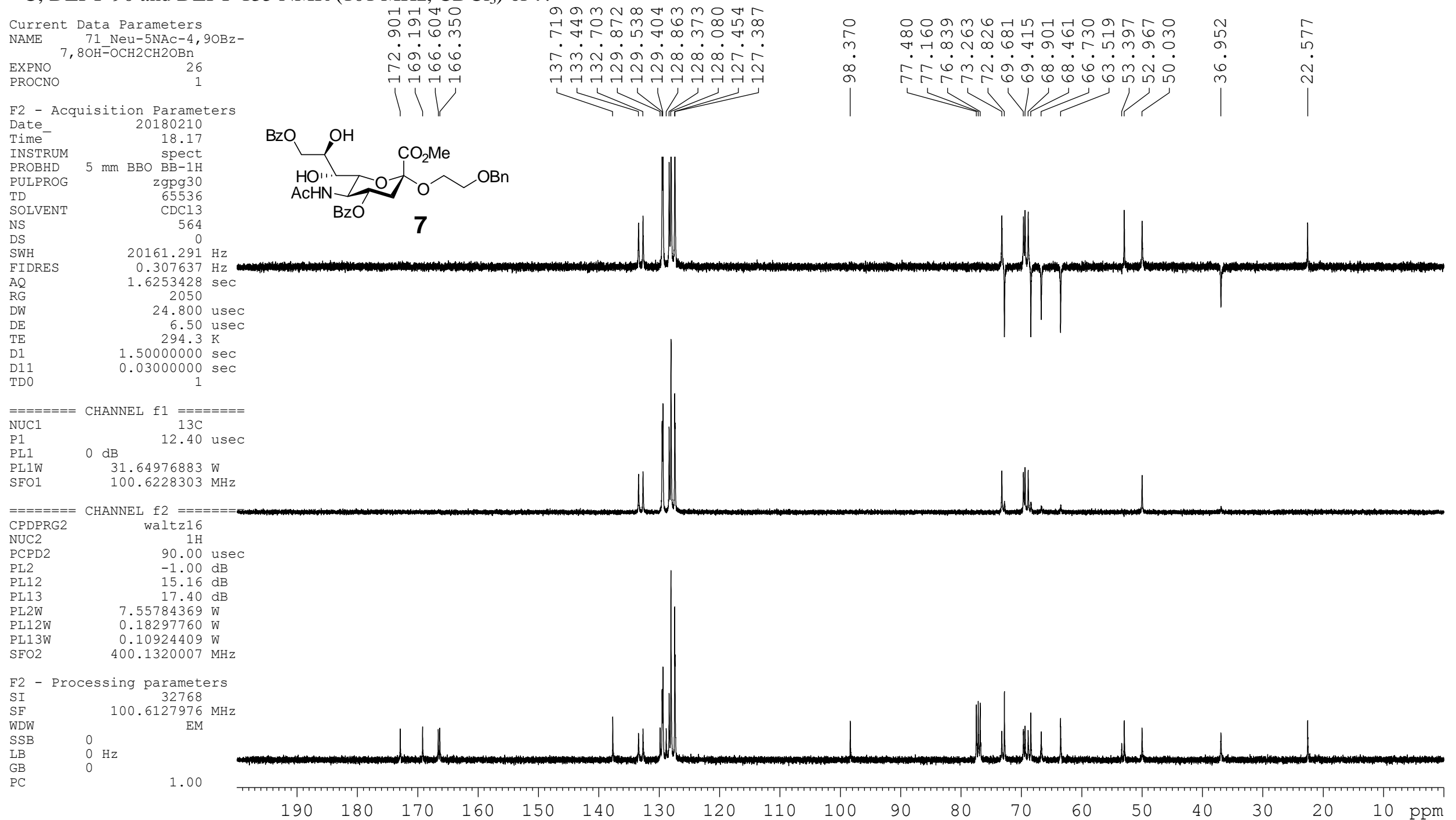




\section{${ }^{1} \mathrm{H}$ NMR $\left(400 \mathrm{MHz}, \mathrm{CDCl}_{3}\right)$ of $\mathbf{8}$ :}

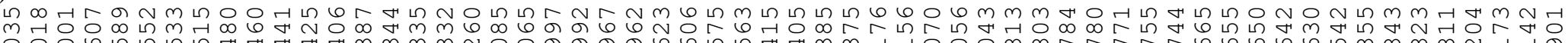
Current Data Parameters

$80 \mathrm{Me}-\overline{7} \mathrm{Neu}-\mathrm{OCHAC}-4,9 \mathrm{OBz}-$ $\begin{array}{lr}\text { EXPNO } & 16 \\ \text { PROCNO } & 1\end{array}$

F2 - Acquisition Parameters

$\begin{array}{ll}\text { Date } & 20171019 \\ \text { Time } & 21.27\end{array}$

Time
INSTRUM

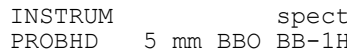

\begin{tabular}{lr} 
PROLPROG & mm BBO $\mathrm{BB}-1 \mathrm{H}$ \\
$\mathrm{TD}$ & $\mathrm{zg} 30$ \\
\hline & 32768
\end{tabular}

TD
SOLVENT

NS

DS
SWH

FIDRES $\quad 6009.615 \mathrm{~Hz}$

$\begin{array}{ll}\text { AQ } & 0.183399 \mathrm{~Hz} \\ & 2.7263477 \mathrm{sec}\end{array}$

$\begin{array}{lr}\text { RG } & 181 \\ \text { DW } & 83.200 \text { usec }\end{array}$

$\begin{array}{lr}\text { DW } & 83.200 \text { usec } \\ \text { DE } & 6.50 \text { usec }\end{array}$

$\begin{array}{lr}\text { TE } & 294.6 \mathrm{~K} \\ \text { D1 } & 1.5000000 \mathrm{sec}\end{array}$

$=======$ CHANNEL $\mathrm{fl}=======-$
NUC1

$\begin{array}{lr}\text { NUC1 } & 1 \mathrm{H} \\ \text { P1 } & 14.00 \text { usec }\end{array}$

$-1.00 \mathrm{~dB}$
PL1W
SE01

SFO1 $400.1326010 \mathrm{MHz}$

F2 - Processing parameters

$\begin{array}{ll}\text { SI } & 32768 \\ \text { SF } & 400.1300096 \\ \text { WDH } & \text { MH }\end{array}$

WDW $\quad$ EM

$\begin{array}{ll}\text { SSB } & 0 \\ \text { LB } & 0 \mathrm{~Hz}\end{array}$

$\mathrm{GB} \quad 0$
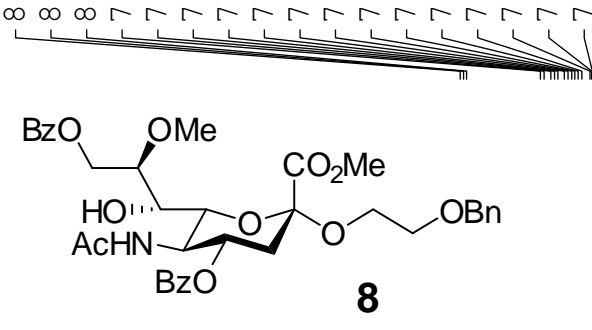

$$
\mid \begin{aligned}
& 0 \\
& 0 \\
& 0 \\
& 0 \\
& 0 \\
& 1 \\
& 1 \\
& 1 \\
& 1
\end{aligned}
$$

ก

m

$\dot{ம}$ ம்ம்ம்ம் சு

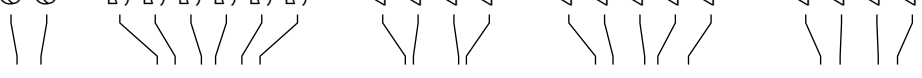

M. Mho

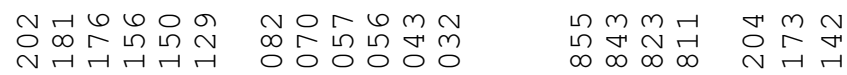

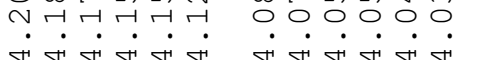

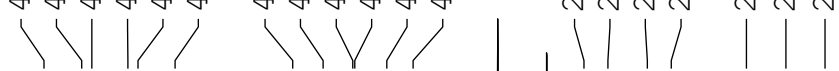

NMn NMG NM N

$\begin{array}{lll}4.2 & \mathrm{ppm} & \mathrm{ppm} \\ \mathrm{ppm} & \mathrm{ppm}\end{array}$

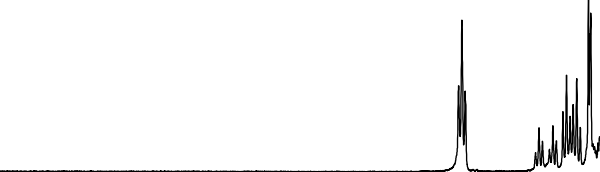

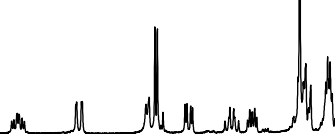

ppm

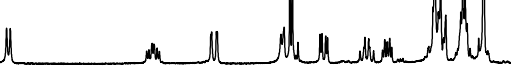

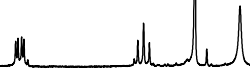

10

9
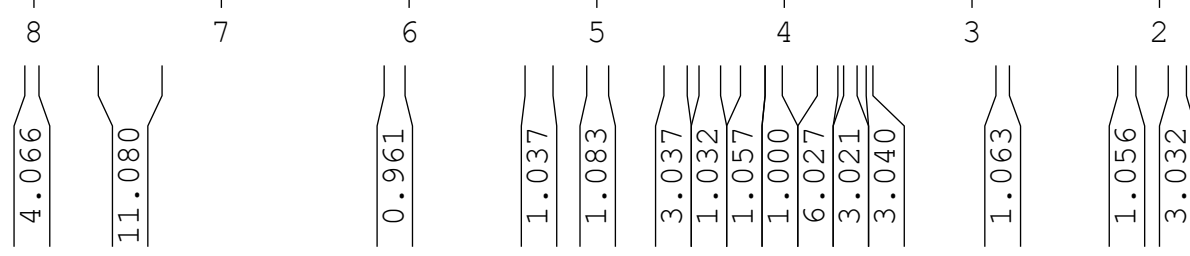

0 ppm 
${ }^{13} \mathrm{C}$, DEPT-90 and DEPT-135 NMR (101 MHz, $\left.\mathrm{CDCl}_{3}\right)$ of $\mathbf{8}$ :

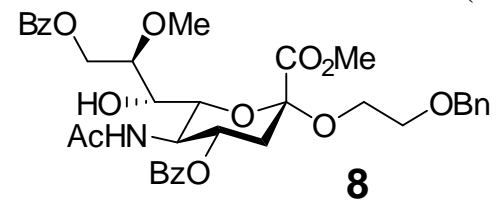

Current Data Parameters
NAME 72 Neu-5NAC-4, $90 \mathrm{Bz}-$ $8 \mathrm{OMe}-\overline{\mathrm{O}} \mathrm{H}-\mathrm{OCH} 2 \mathrm{CH} 2 \mathrm{OBn}$

EXPNO
PROCNO

F2 - Acquisition Parameters

Date_r 2017102

TimSTRUM

PROBHD

PULPROG
TD

SOLVE

NS

SWH
FIDRES

$\mathrm{AQ}$

RG

$\mathrm{DW}$
$\mathrm{TE}$
$\mathrm{D}$

D1
D11

D11
TD0

$===$
NUC1
P1

P1
PL1
PL1

PL1W

$=======$
CPDPRG2

CPDPR
NUC2
PCPD2

PCPD2

PL12

PL13
PL2W

PL12W

SFO2

F2

SF $\quad 100.6127886$ $0 \mathrm{~Hz}$

$20161.291 \mathrm{~Hz}$ $1.6253428 \mathrm{sec}$

2050
24.800 usec

$.50000000 \mathrm{sec}$

$31.64976883 \mathrm{~W}$
$100.6228303 \mathrm{MHz}$

HANNEL $f 2=======$

90.00 usec

$-1.00 \mathrm{~dB}$

$15.16 \mathrm{~dB}$

$7.55784369 \mathrm{~W}$

ing parameter
32768
100.6127886

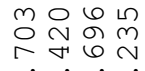

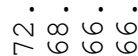

가이

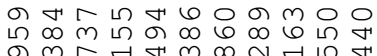

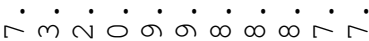

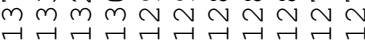

急

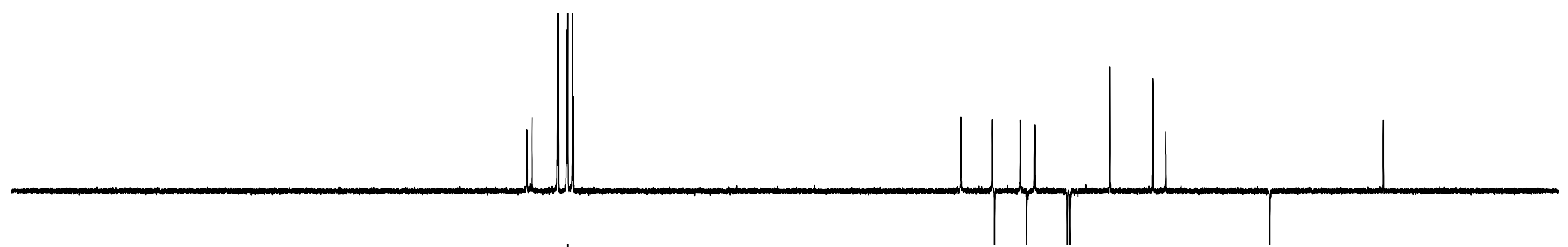

$\mathrm{f} 1=======$
$13 \mathrm{C}$
12.40 use

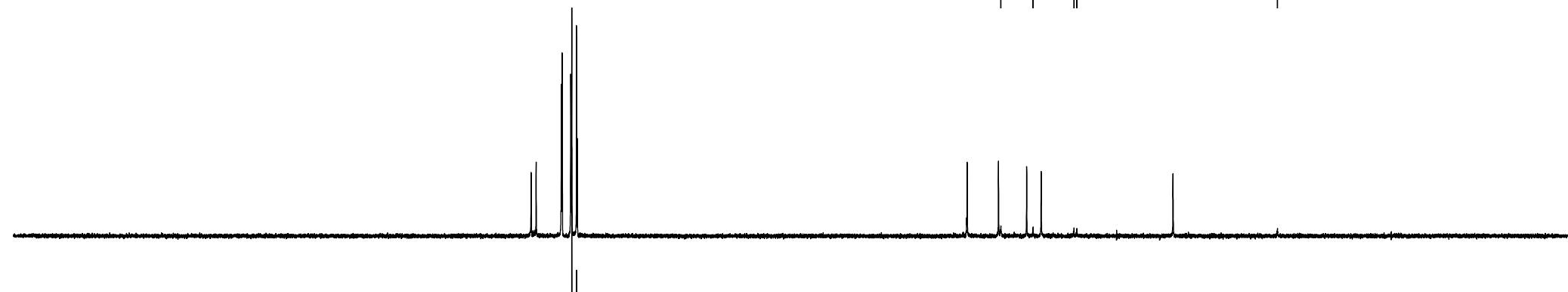

150

140

30

120

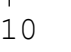

100

90

80

1.00

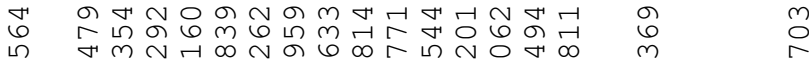

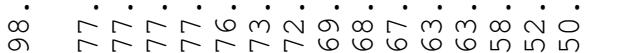

$\longrightarrow$ VI

| 


\section{${ }^{1} \mathrm{H}$ NMR $\left(400 \mathrm{MHz}, \mathrm{CDCl}_{3}\right)$ of $\mathbf{9}$}

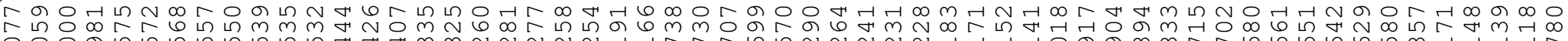

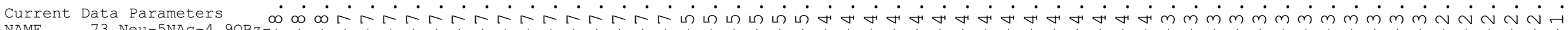

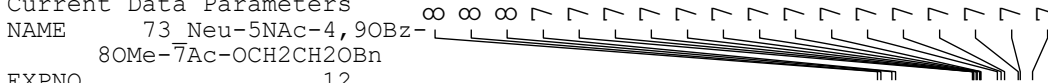

EXPNO
PROCNO

F2 - Acquisition Parameters

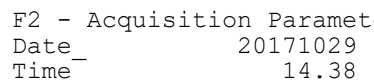

INSTRUM
PROBHD

PULPROG

TD

SOLVENT
NS

SWH

FIDRES

$\mathrm{AQ}$
$\mathrm{RG}$

RG

$\mathrm{DE}$

D1 0

$=======$ CHANNEL $\mathrm{fl}========$
NUC1
$1 \mathrm{H}$

$\begin{array}{lr}\text { NUC1 } & 1 \mathrm{H} \\ \mathrm{P} 1 & 14.00 \\ \mathrm{PL} 1 & -1.00 \mathrm{usec}\end{array}$

$\begin{array}{lr}\text { PL1 } & -1.00 \mathrm{~dB}\end{array}$

$\begin{array}{lr}\text { PL1W } & 7.55784369 \mathrm{~W} \\ \text { SFO1 } & 400.1326010 \mathrm{MHz}\end{array}$

F2 - Processing parameters

$\begin{array}{ll}\text { SI } & 32768 \\ \text { SF } & 400.1300096 \mathrm{MHz}\end{array}$

$\begin{array}{ll}\text { SF } & 400.1300096 \mathrm{M} \\ \text { WDW } & \text { O }\end{array}$

$\begin{array}{ll}\text { SSB } & 0 \\ \text { LB } & 0 \\ \text { Hz }\end{array}$

GB $\quad 0$

1.00

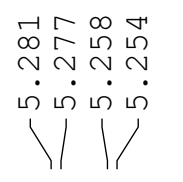

ம் ம் ம் ம் ம்

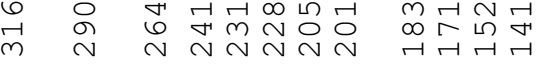

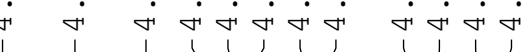

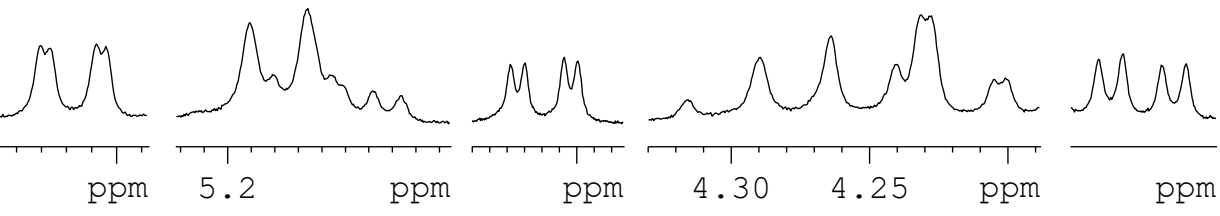

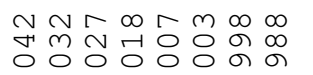

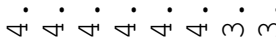

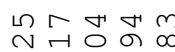

नू नुㅇํ

$m m m n$

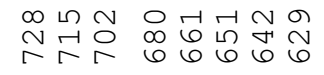

$m m m \dot{m} m \dot{m}$

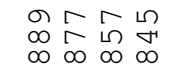

$\sim \sim \sim \sim$

111

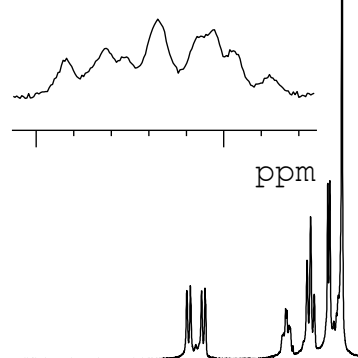

NMh

ppm
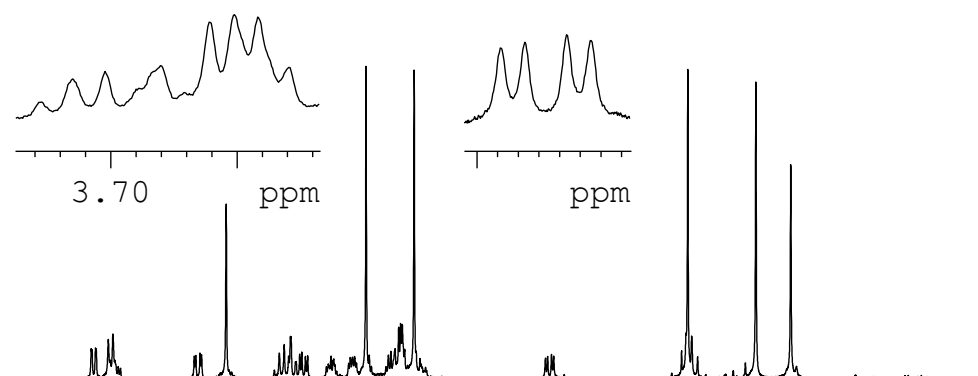
5

4

3

2

1

0 ppm

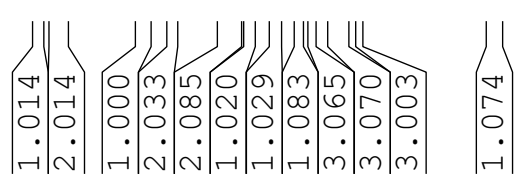

\begin{tabular}{cc|c}
1 & 1 \\
0 & & \\
$m$ & -1 \\
0 & -1 \\
0 & 0 \\
0
\end{tabular} 
${ }^{13} \mathrm{C}$, DEPT-90 and DEPT-135 NMR (101 MHz, $\left.\mathrm{CDCl}_{3}\right)$ of $\mathbf{9 :}$

$\mathrm{BzO} \mathrm{OMe} \mathrm{CO}_{2} \mathrm{Me}$
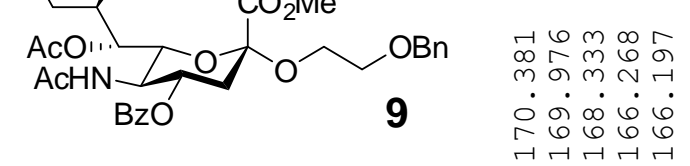

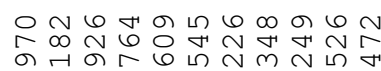

$\dot{m} \dot{m} \dot{m} \dot{m} \dot{\sim} \dot{\sim} \dot{\sim} \dot{\sim} \dot{\sim} \stackrel{\infty}{\sim} \stackrel{\sim}{\sim} \hat{\sim}$

$\underset{N}{\infty} \quad$ o

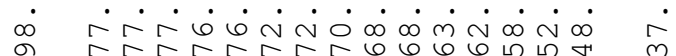

SN

Current Data Parameters
NAME 73 Neu-5NAC-4, $90 \mathrm{Bz}$

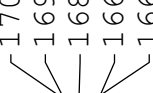

$80 \bar{M}=-7 \mathrm{AC}-\mathrm{OCH} 2 \mathrm{CH} 2 \mathrm{OBn}$

EXPNO
PROCNO

F2 - Acquisition Parameters

Date Time $^{-} \quad 20171029$

spect
INSTRUM
PROBHD

PULPROG

TD

SOLVEN

20161.291

$\begin{array}{lr}20161.291 \mathrm{~Hz} \\ \text { FIDRES } & 0.307637 \mathrm{~Hz}\end{array}$

$\begin{array}{ll}\mathrm{AQ} & 1.6253428 \mathrm{sec} \\ \mathrm{RG} & 2050 \mathrm{sec}\end{array}$

$\begin{array}{rr}\mathrm{DW} & 24.800 \text { usec } \\ \mathrm{DE} & 6.50 \text { usec }\end{array}$

$295.1 \mathrm{~K}$

$\begin{array}{ll}\text { D1 } & 1.50000000 \mathrm{sec} \\ \text { D11 } & 0.03000000 \mathrm{sec}\end{array}$

TDO

$=======$ CHANNEL $\mathrm{fl}=======$
NUC1 $13 \mathrm{C}$
P1
PL1

$\begin{array}{lll}\text { PL1 } & 0 \mathrm{~dB} \\ \text { PL1W } & 31.64976883 \mathrm{~W}\end{array}$

$\begin{array}{ll}\text { PL1W } & 31.64976883 \mathrm{~W} \\ \text { SFO1 } & 100.6228303 \mathrm{MHz}\end{array}$

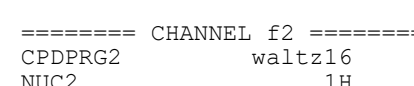

$\begin{array}{ll}\text { NUC2 } & 1 \mathrm{H} \\ \text { PCPD2 } & 90.00 \mathrm{usec}\end{array}$

PL1 2
PL12

PL13

PL12W

SFO2

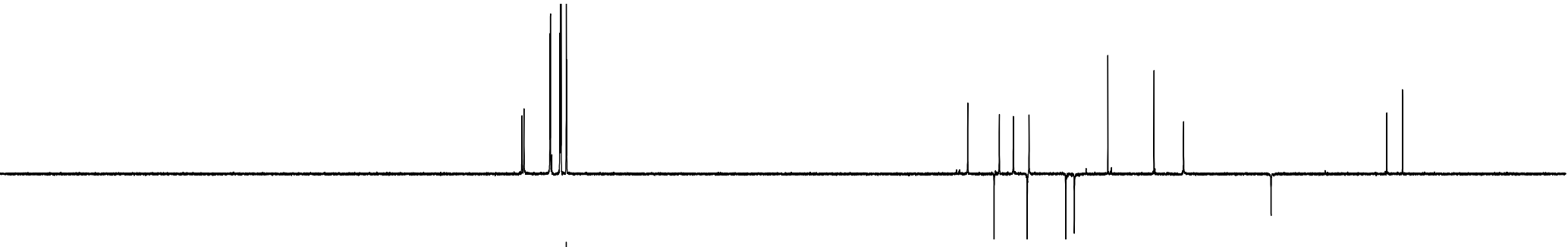

sing parameters

SI Processing para 32768

$-1.00 \mathrm{~dB}$
$15.16 \mathrm{~dB}$

$17.40 \mathrm{~dB}$

7.55784369 W

0.18297760 W

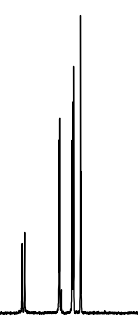

제에

ล่ำ

1

$\begin{array}{lll}\mathrm{SEN} & \mathrm{EM} \\ \mathrm{SSB} & 0 & \end{array}$ $\mathrm{Hz}$

$190-180-170=160$
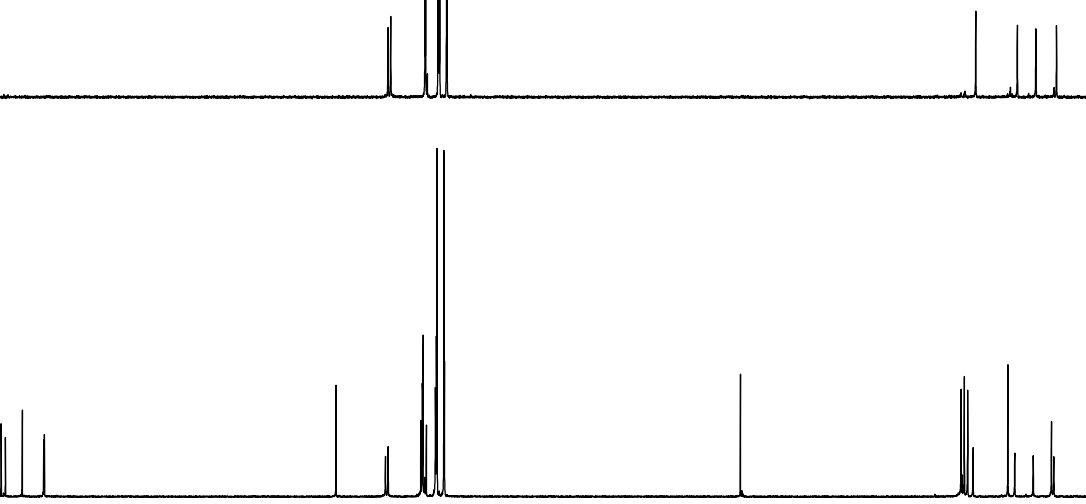

1.00 


\section{${ }^{1} \mathrm{H}$ NMR $\left(400 \mathrm{MHz}, \mathrm{CDCl}_{3}\right)$ of 2:}

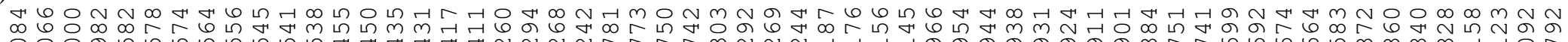

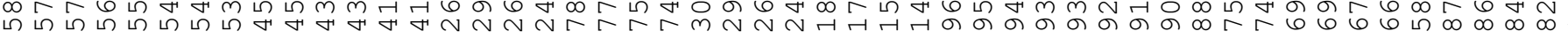

Current Data Parameters $74 \mathrm{Neu}-5 \mathrm{NAC}-4,90 \mathrm{O} 2-10$
$8 \mathrm{Me}-7 \mathrm{AC}-\mathrm{OCH} 2 \mathrm{CH} 2 \mathrm{OH}$ EXPNO
PROCNO

F2 - Acquisition Parameters

$\begin{array}{lr}\text { Date_ } & 20180121 \\ \text { Time- } & 16.25\end{array}$

INSTRUM

PROBHD

TD

$5 \mathrm{~mm}$ BBO $\begin{array}{r}\text { spect } \\ \mathrm{BB}-1 \mathrm{H} \\ \mathrm{zg} 30\end{array}$

SOLVE

$\mathrm{CDC13}$

DS

FIDRES

$6009.615 \mathrm{~Hz}$

$0.183399 \mathrm{~Hz}$

$0.7263477 \mathrm{sec}$
36

83.200 usec

$\begin{array}{lr}\text { DW } & 83.200 \text { use } \\ D E & 6.50 \text { use } \\ \text { TE } & 293.9 \mathrm{~K}\end{array}$

D1 $\quad 1.50000000 \mathrm{sec}$

$=======$ CHANNEL $\mathrm{f} 1========$
NUC1 $1 \mathrm{H}$
P1 14.00 usec
PL1W

PL1 $\quad-1.00 \mathrm{~dB}$

$\begin{array}{lr}\text { PL1W } & 7.55784369 \mathrm{~W} \\ \text { SFO1 } & 400.1326010 \mathrm{MHz}\end{array}$

F2 - Processing parameters

F2 - Processing parameters

SE $\quad 400.1300095 \mathrm{MHZ}$

WDW

$\begin{array}{lll}\text { SSB } & 0 \\ \text { LB } & 0 & \mathrm{~Hz}\end{array}$

GB
PC

1.00

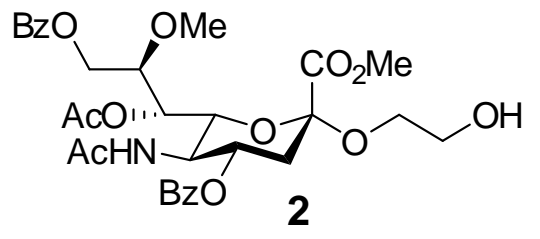

स $\infty N 6$ 几

ํํำ

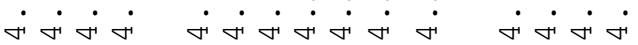

$\mathrm{AcHN} \sim \mathrm{O}$

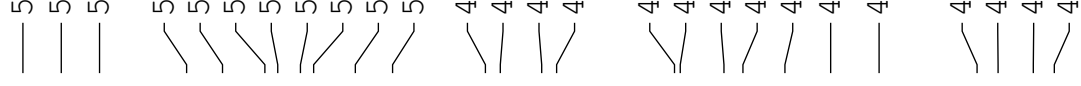

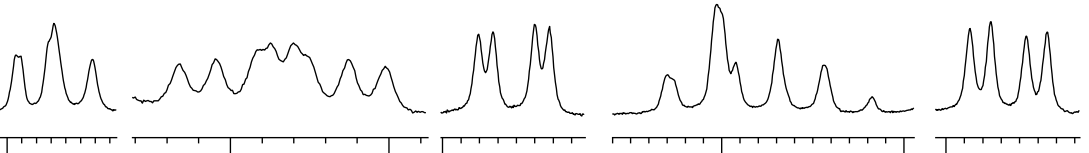

$\begin{array}{lllllll}\text { ppm } 5.20 \quad \mathrm{ppm} & \mathrm{ppm} & 4.3 & \mathrm{ppm} & \mathrm{ppm}\end{array}$

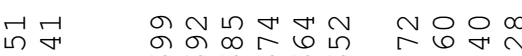

r.

$m m m m m m \sim N \sim \sim$

11111111

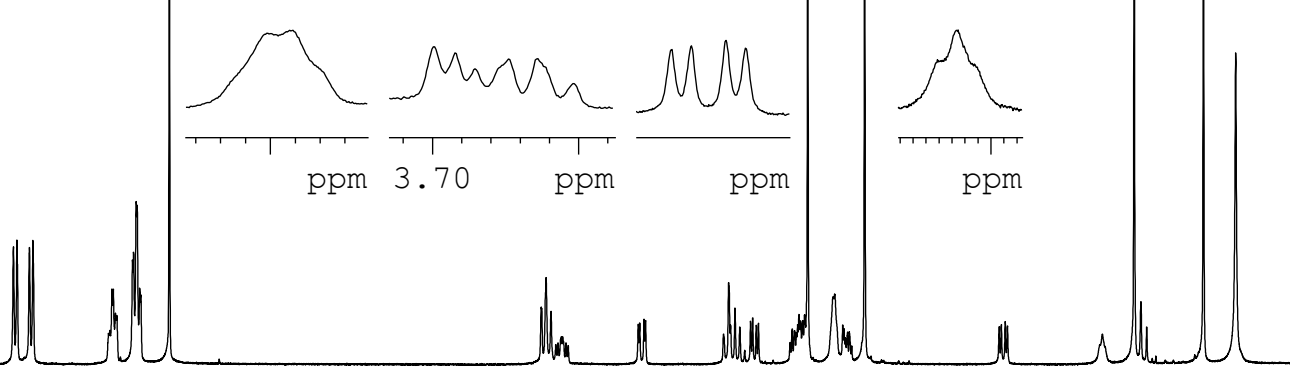

$m \sim n$

$\dot{\sim} \dot{\sim} \dot{\sim}$

11

10

9

8

5

4

3

2

1

0 ppm

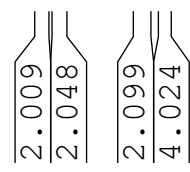

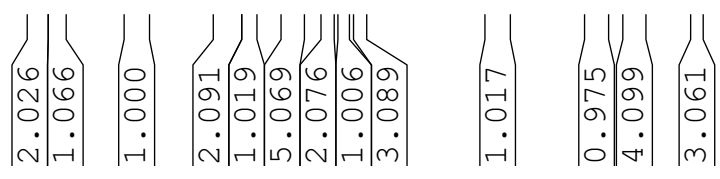


${ }^{13} \mathrm{C}$, DEPT-90 and DEPT-135 NMR (101 MHz, $\mathrm{CDCl}_{3}$ ) of $\mathbf{2}$ :

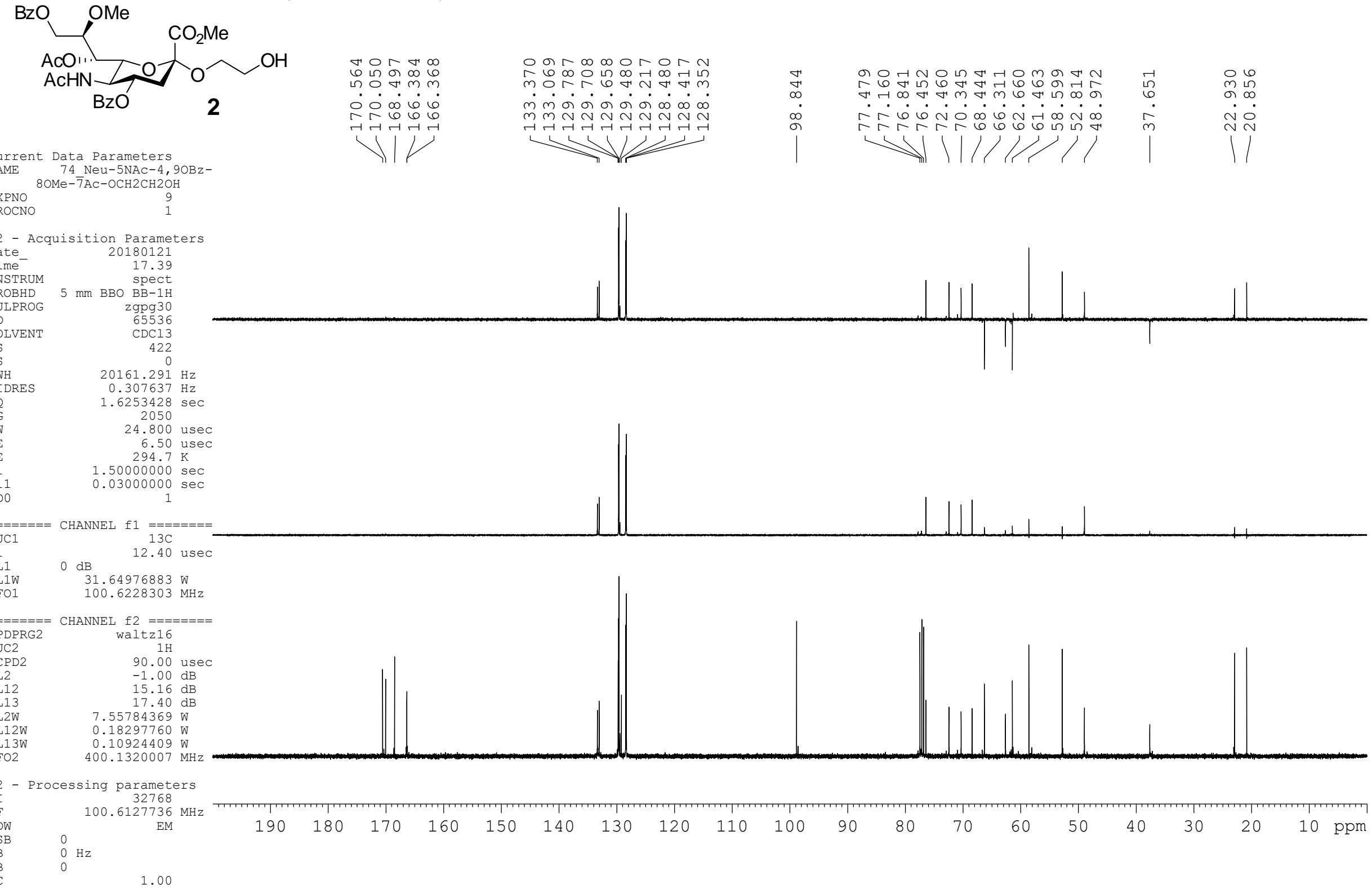


${ }^{1} \mathrm{H}$ NMR $\left(400 \mathrm{MHz}, \mathrm{CDCl}_{3}\right)$ of $\mathbf{S 2}$ :

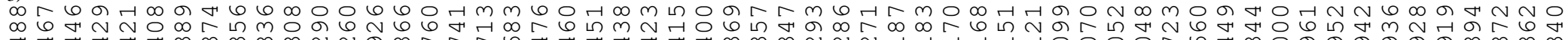

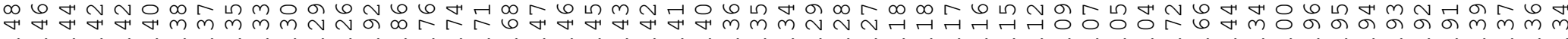

Current Data Parameters $89 \mathrm{CHPh}-\overline{7} \mathrm{OH}-\mathrm{SPh}$

EXPNO
PROCNO

F2 - Acquisition Parameters

F2 - Acquisition Parameter
Date_ 20180301

Time-

INSTRUM
PROBHD

PROBHD
PULPROG

SOLVENT

NS

DS

FIDRES

$\mathrm{AQ}$
$\mathrm{RG}$

RG

$\mathrm{DW}$
$\mathrm{DE}$

$\mathrm{DE}$
$\mathrm{TE}$
$\mathrm{D} 1$

DI
TDO

$=======$ CHANNEL $\mathrm{f} 1 \mathrm{l}=======$

P1

PL1W

$7.55784369 \mathrm{~W}$
$400.1326010 \mathrm{MHz}$

F2 - Processing parameters

$\begin{array}{lr}\text { SI } & 32768 \\ \text { SF } & 400.1300098 \\ \text { MHz }\end{array}$

WDW $\quad 400.1300098$

$\begin{array}{lll}\mathrm{SSB} & 0 \\ \mathrm{~TB} & 0 & 0\end{array}$

$\mathrm{Hz}$

$6009.615 \mathrm{~Hz}$ $0.183399 \mathrm{~Hz}$
$2.7263477 \mathrm{sec}$ 83.200 usec 6.50 use $294.1 \mathrm{~K}$

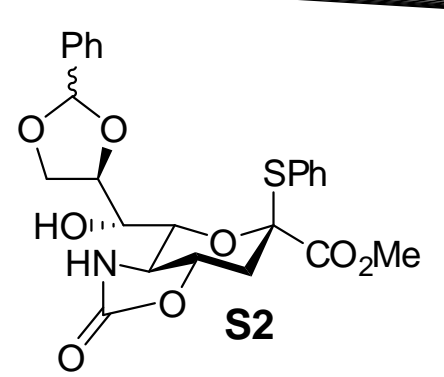

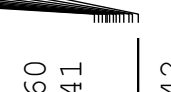

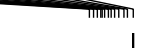

NNHmmNmm

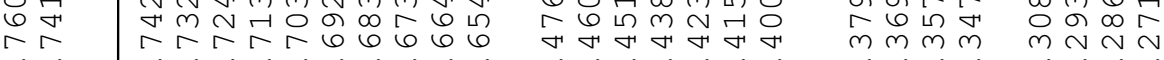
ம்

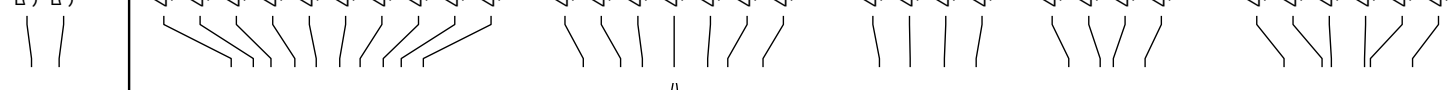
$\cdot \cdot \cdot \cdot \cdot \cdot$

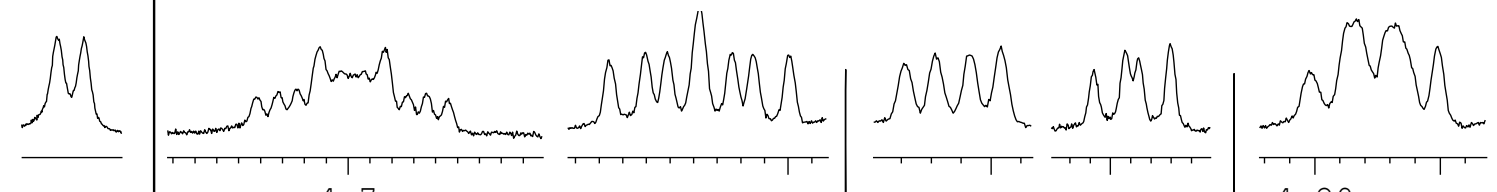

ppm

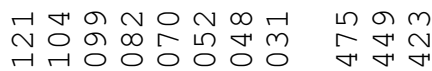

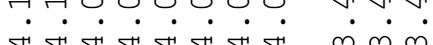
$\int_{1}^{1 / 1}$

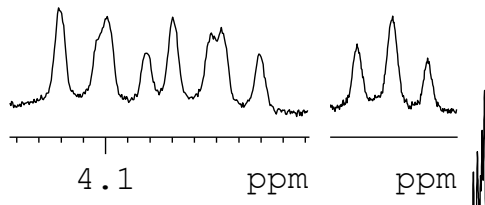

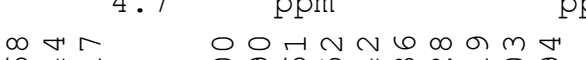

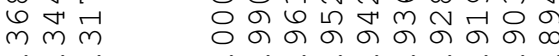

$\dot{m} \dot{m} \quad \dot{m} \dot{\sim} \dot{\sim} \dot{\sim} \dot{\sim} \dot{\sim} \dot{\sim}$

1111111

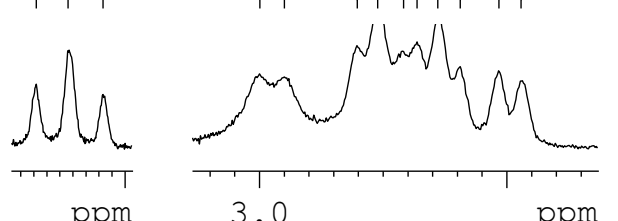$$
\text { ppm ppm }
$$

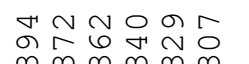

$\dot{\sim} \dot{\sim} \dot{\sim} \dot{\sim} \dot{\sim}$

1111

$$
\text { 4.20 ppm }
$$
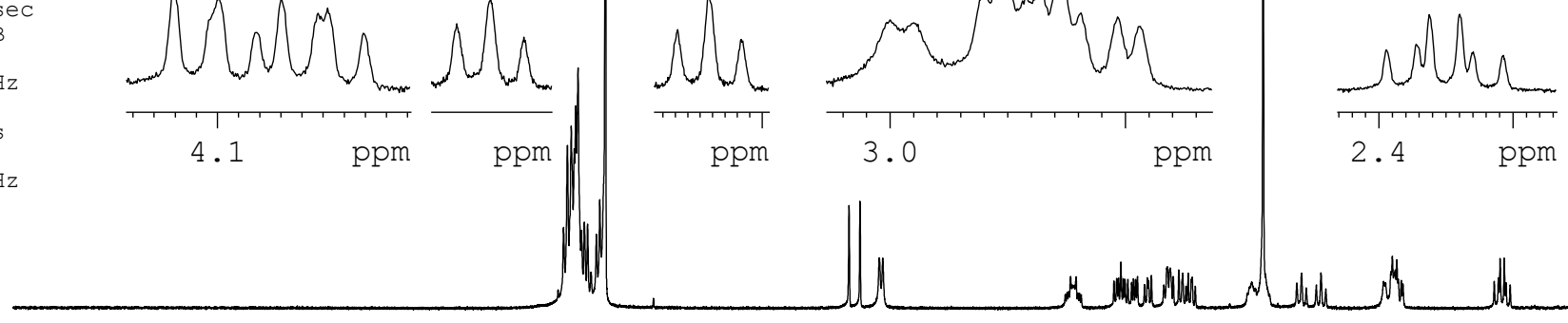

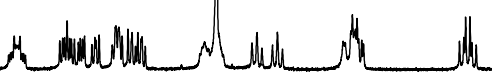
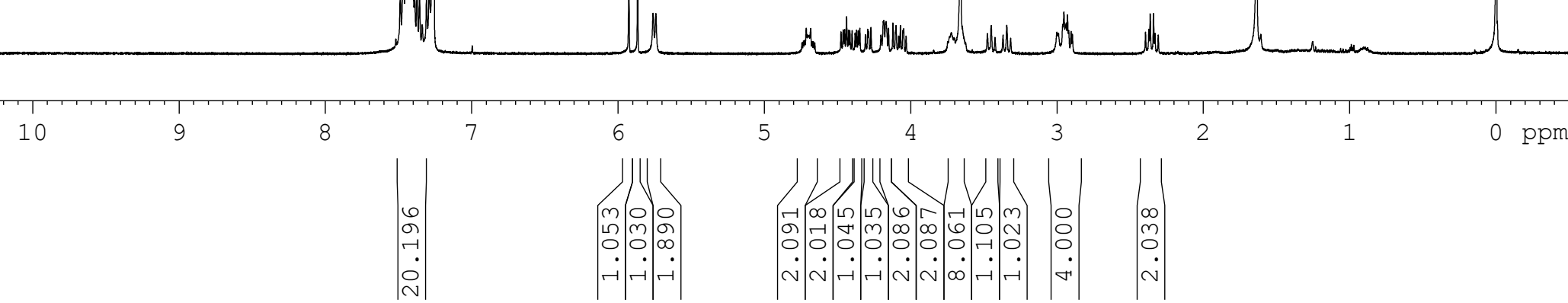
${ }^{1} \mathrm{H}$ NMR (400 MHz, $\mathrm{CDCl}_{3}$ ) of $\mathbf{S 3}$ :

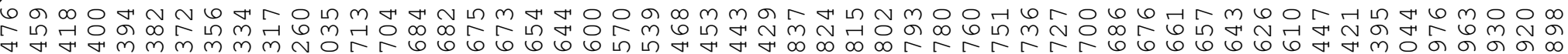

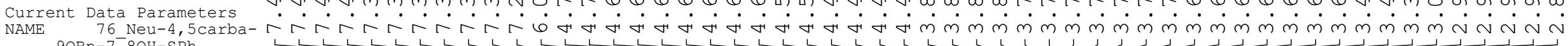
EXPNO PROCNO

F2 - Acquisition Parameters

Date_

Time 20.30

PROBHD $5 \mathrm{~mm}$ BBO $\mathrm{BB}-1 \mathrm{H}$

PULPROG

TD

NS
DS

$\mathrm{BB}-1 \mathrm{H}$
$\mathrm{zg} 30$

$\mathrm{CDCl} 3$

SWH

$A Q$
$R G$

6009.615

$0.183399 \mathrm{~Hz}$

$2.7263477 \mathrm{sec}$

83.287 usec

$\begin{array}{lr}\text { DW } & 83.200 \text { usec } \\ \text { DE } & 6.50 \text { usec } \\ \text { TE } & 294.0 \mathrm{~K} \\ \text { D1 } & 1.50000000 \mathrm{sec}\end{array}$

$\begin{array}{lr}\text { DE } & 6.50 \mathrm{usec} \\ \mathrm{TE} & 294.0 \mathrm{~K} \\ \mathrm{D} & 1.50000000 \mathrm{sec}\end{array}$

TD0

N

CHANNEL $\mathrm{f}$

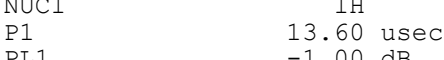

PL1 $\quad-1.00 \mathrm{~dB}$

PL1W

F2 - Processing parameters

SI $\quad 400.1300095 \mathrm{MHz}$

WDW

$\begin{array}{ll}\text { SSB } & 0 \\ \text { LB } & 0 \\ \text { GB } & 0\end{array}$

$\mathrm{BnO}$

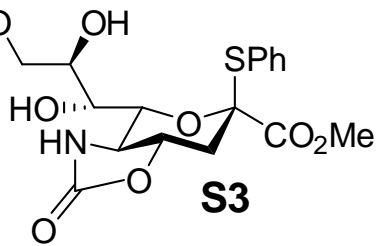

단다요

을

$\begin{array}{llll}\circ & 0 & 6 & -1 \\ 0 & 0 & 6 & 6 \\ 0 & 0 & 6 & 6\end{array}$

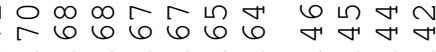

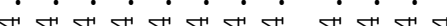

$\dot{m} \dot{m} \dot{m} \cdot$

$\dot{m} \dot{m} \dot{m}$

$\dot{m} \dot{m}$

$1+$

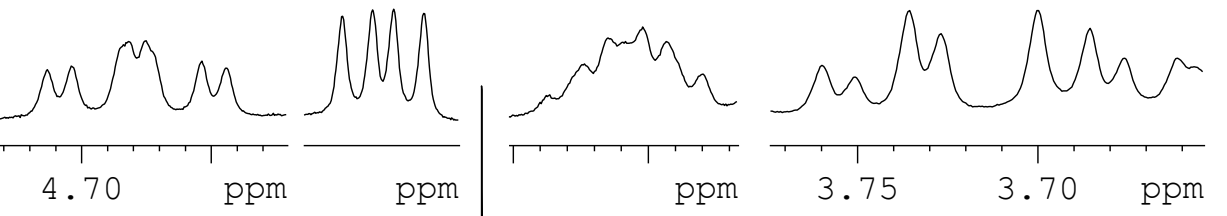

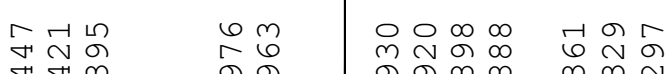

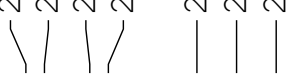

MU M

MM UU

ppm

1

1.00

10

9

8

$\left|\begin{array}{l}\mid \\ \\ 6 \\ +1 \\ -1 \\ 0 \\ 0 \\ -1\end{array}\right|$
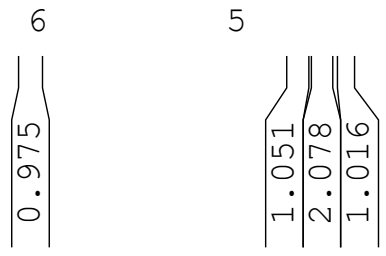

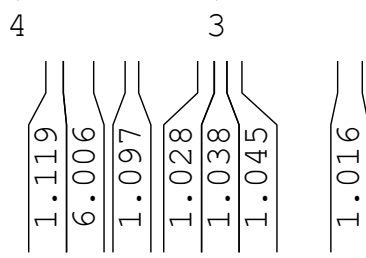

ppm 


\section{${ }^{1} \mathrm{H}$ NMR (400 MHz, $\mathrm{CDCl}_{3}$ ) of $\mathbf{S 4 :}$}

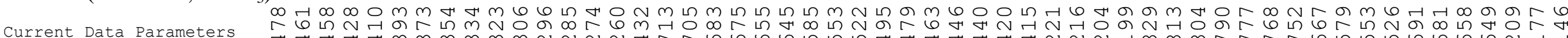
NAME EXPNO $90 \mathrm{Bn}-7,8 \mathrm{X}-\mathrm{SPh}$

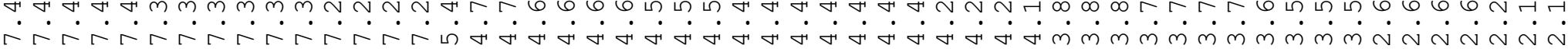
PROCNO

F2 - Acquisition Parameters

Date_ 20180102

INSTRUM

PROBHD

$5 \mathrm{~mm}$ BBO $\mathrm{BB}-1 \mathrm{H}$

PULPROG
TD

SOLVENT

NS

DS

FIDRES

AQ

DW

$\mathrm{DW}$
$\mathrm{DE}$
$\mathrm{TE}$
$\mathrm{D}$

D1 0

$=======$ CHANNEL $\mathrm{f}$

P1

PLIW

$\begin{array}{lr}\text { PL1W } & 7.55784369 \mathrm{~W} \\ \text { SFO1 } & 400.1326010 \mathrm{MHz}\end{array}$

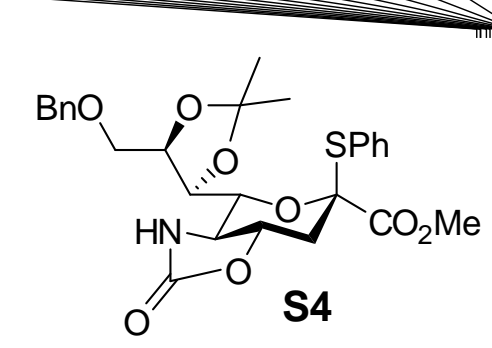

$m \backsim m \backsim \backsim \backsim$ ก

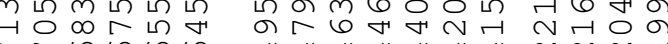

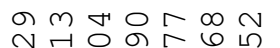

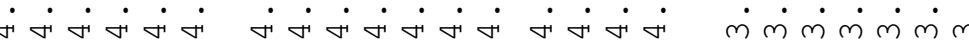

$\mathrm{BB}-1 \mathrm{H}$
$\mathrm{zg} 30$
32768

$\mathrm{CDC}$
64

$6009.615 \mathrm{~Hz}$ $2.7263477 \mathrm{sec}$

83.2000

3.200 usec
6.50 usec

$1.50000000 \mathrm{sec}$

Processing parameters

$\begin{array}{lr}\text { SI } & 32768 \\ \text { SF } & 400.1300098 \mathrm{MHz}\end{array}$

$\begin{array}{lr}\text { SF } & 400.1300098 \mathrm{MHz} \\ \text { WDW } & \text { EM }\end{array}$

$\mathrm{SSB} \quad 0$

GB

$\mathrm{O}^{\mathrm{O}} \mathrm{Hz}$

1.00

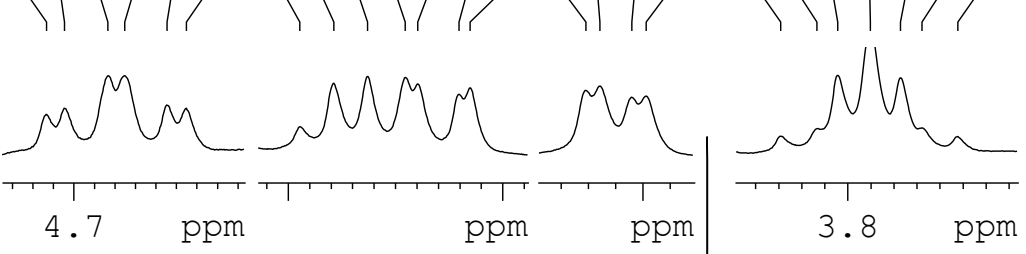

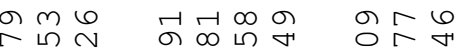

감

$\dot{m} \dot{m} \quad \dot{\sim} \dot{\sim} \dot{\sim} \quad \dot{\sim} \dot{\sim} \dot{\sim}$

1111111

na ma Nu

ppm $2.7 \mathrm{ppm}$

ppm

Ninl 1
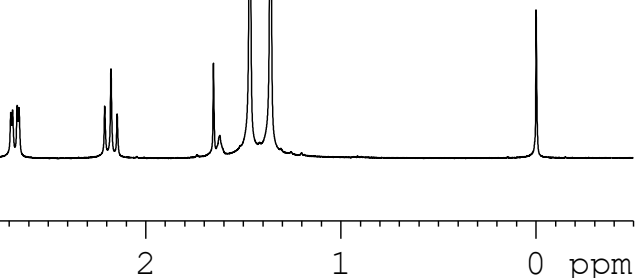

10

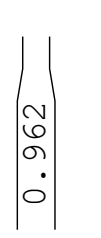

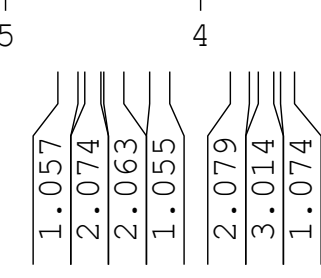

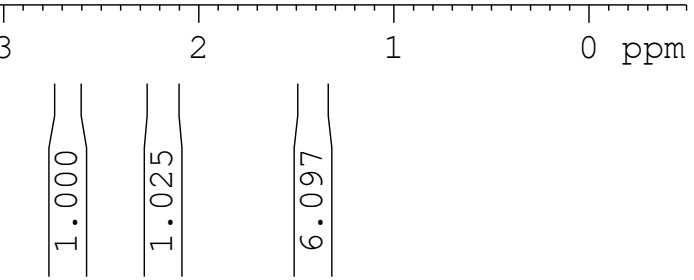




\section{${ }^{1} \mathrm{H}$ NMR (400 MHz, $\mathrm{CDCl}_{3}$ ) of $\mathbf{2 0} \boldsymbol{\alpha}$ :}

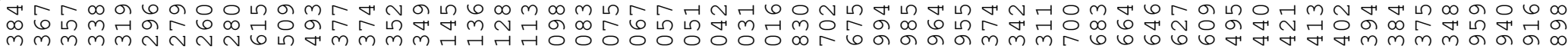

Current Data Parameters $08 \mathrm{-}-\overline{7}, 8 \mathrm{X}-\mathrm{OPO}(\mathrm{OBu}) 2$

$\begin{array}{lr}\text { EXPNO } & \text { POBn-7, } \\ \text { PROCNO } & 1\end{array}$

F2 - Acquisition Parameters Date_ 20180314

INSTRUM spect

PROBHD $5 \mathrm{~mm}$ BBO BB-1H

PULPROG

SOLVENT

DS

FIDRES

$A Q$

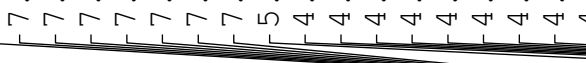

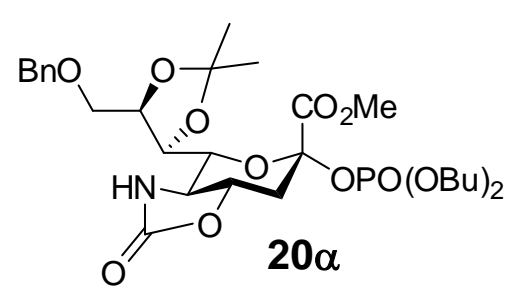

$0.7263477 \mathrm{sec}$
181

83.200 usec

$\begin{array}{lr}\mathrm{DE} & 6.50 \mathrm{usec} \\ \mathrm{TE} & 294.4 \mathrm{~K}\end{array}$

TDO

$=======$ CHANNEL

P1

PL1

SFO1 $=======$
$1 \mathrm{H}$ 14.00 usec $-1.00 \mathrm{~dB}$
$7.55784369 \mathrm{~W}$ $400.1326010 \mathrm{MHz}$

F2 - Processing parameters

SI $\quad 400.1300092$

EM MH

$\begin{array}{ll}\mathrm{SSB} & 0 \\ \mathrm{LB} & 0 \mathrm{~Hz}\end{array}$

GB

$\mathrm{O} \mathrm{Hz}$

1.00

(1)

10

9

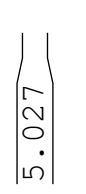

7

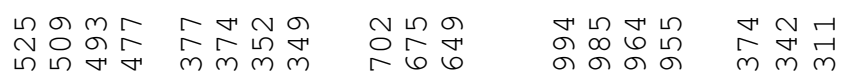
मां

111111120

Mh Ju Nh MM MU

$3.0 \mathrm{ppm} \quad \mathrm{ppm}$

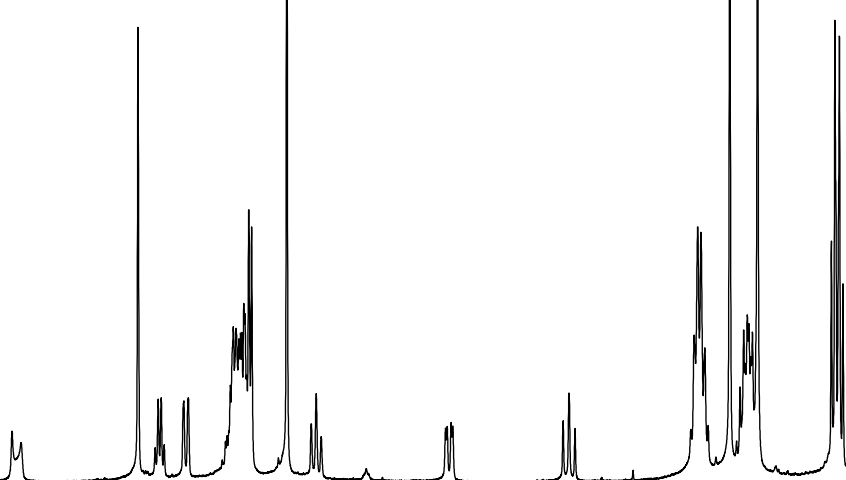

2

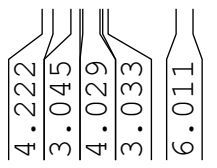


${ }^{1} \mathrm{H}$ NMR $\left(400 \mathrm{MHz}, \mathrm{CDCl}_{3}\right)$ of $\mathbf{2 0} \boldsymbol{\beta}$ :

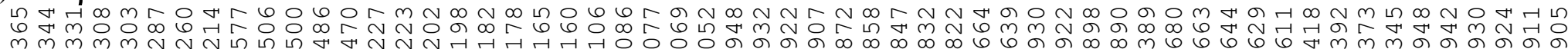

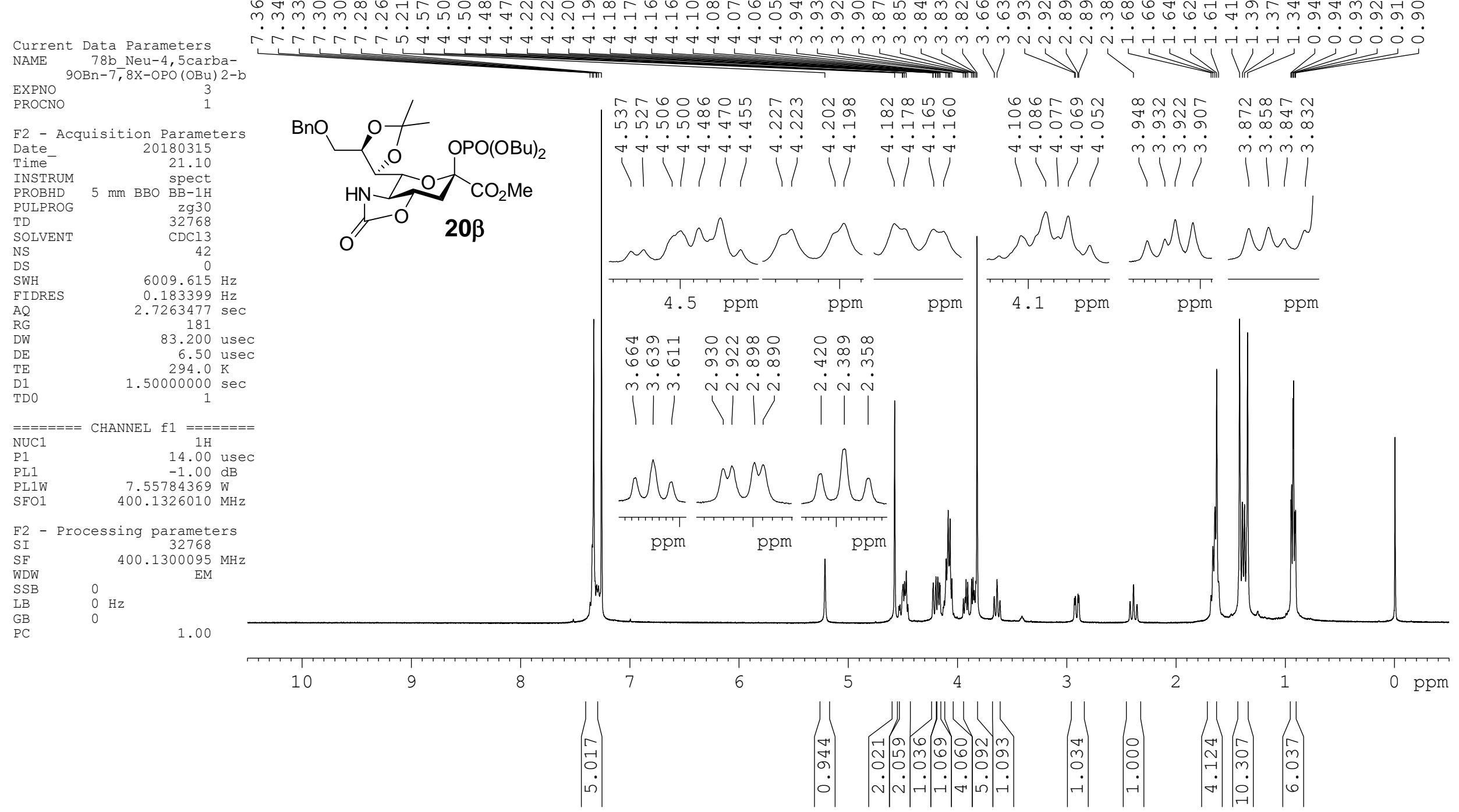




\section{${ }^{1} \mathrm{H}$ NMR (400 MHz, $\left.\mathrm{CDCl}_{3}\right)$ of $\mathbf{S 6}$ :}

Current Data Parameters

NAME 85_Glu-2, 3, 4, 60AC-SPh EXPNO 2
1

F2 - Acquisition Parameters

$\begin{array}{lr}\text { Date } & 20180213 \\ \text { Time } & 17.50\end{array}$

INSTRUM spect

$\begin{array}{lr}\text { PROBHD } & 5 \mathrm{~mm} \text { BBO } \mathrm{BB}-1 \mathrm{H} \\ \text { PULPROG } & \mathrm{zg} 30 \\ \mathrm{TD} & 32768\end{array}$

SOLVENT

SOLV
NS

DS

$\begin{array}{ll}\text { SWH } & 6009.615 \mathrm{~Hz} \\ \text { FIDRES } & 0.183399 \mathrm{~Hz}\end{array}$

$\begin{array}{lr}\mathrm{AQ} & 2.7263477 \mathrm{sec}\end{array}$

$\begin{array}{lr}\text { RG } & 144 \text { usec } \\ \text { DW } & 63.200 \text { usec }\end{array}$

6.50 use
$293.6 \mathrm{~K}$

$\begin{array}{lr}\text { TE } & 293.6 \mathrm{~K} \\ \text { D1 } & 1.50000000 \mathrm{sec} \\ \text { TD0 } & 1\end{array}$

$=======$ CHANNEL $\mathrm{fl}========$
NUC1 $1 \mathrm{H}$

P1

PL1 14.00 usec

PL1W 7.55784369 W

$400.1326010 \mathrm{MHz}$

F2 - Processing parameters

SI - Processing parameters

$\begin{array}{ll}\text { SI } & 400.1300094 \mathrm{MHz}\end{array}$

$\begin{array}{ll}\text { SSB } & 0 \\ \text { IB } & 0\end{array}$

$\begin{array}{lll}\text { LB } & 0 \mathrm{~Hz} \\ \text { GB } & 0\end{array}$

$\mathrm{PC}$

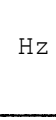

$\mathrm{EM}$

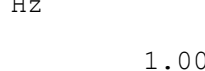

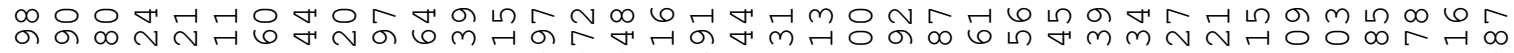

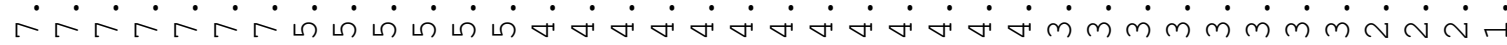
$\longrightarrow 1 /$
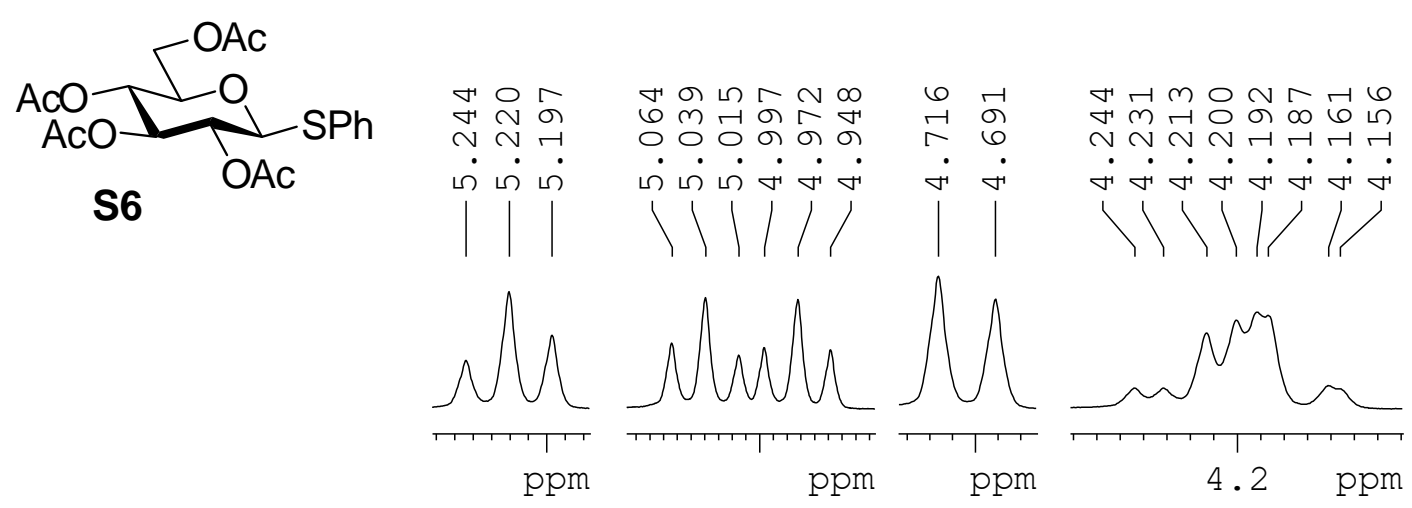

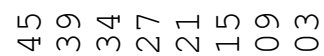

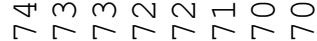

$\dot{m} \dot{m} \dot{m} \dot{m} \dot{m} \dot{m} \dot{m} \dot{m}$

S6

ppm

ppm

ppm

4.2 $\mathrm{ppm}$
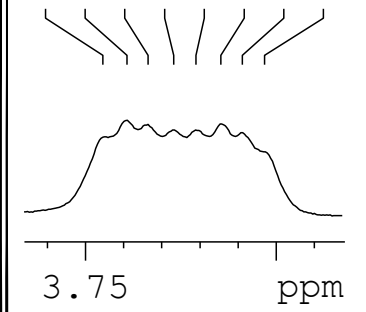

T11

9

8

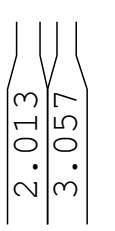

6

5

3

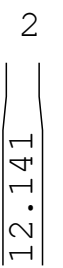


${ }^{1} \mathrm{H}$ NMR (400 MHz, $\mathrm{CDCl}_{3}$ ) of $\mathbf{S 7}$ :

Current Data Parameters
NAME 86 Glu-4, 6CHPh-

NAME
EXPNO

PROCNO

F2 - Acquisition Parameters

$\begin{array}{lr}\text { Date } & 20170911 \\ \text { Time } & 16.00\end{array}$

INSTRUM $\quad$ spect

PROBHD $5 \mathrm{~mm}$ BBO BB-1H

$\begin{array}{lr}\text { PULPROG } & \text { zg30 } \\ \text { TD } & 32768\end{array}$

$\begin{array}{ll}\text { TD } & 32768 \\ \text { SOLVENT } & \text { CDC13 } \\ \text { DS } & 15\end{array}$

$\begin{array}{lr}\text { NS } & 15 \\ \text { DS } & 0\end{array}$

$\begin{array}{ll}\text { DS } & 6009.615 \mathrm{~Hz}\end{array}$

FIDRES $\quad 0.183399 \mathrm{~Hz}$

$\begin{array}{lr}\text { AQ } & 2.7263477 \mathrm{sec} \\ \text { RG } & 228\end{array}$

$\begin{array}{lr}\text { RG } & 228 \\ \text { DW } & 83.200 \text { usec }\end{array}$

$\begin{array}{lr}\mathrm{DE} & 6.50 \mathrm{usec} \\ \mathrm{TE} & 296.6 \mathrm{~K}\end{array}$

D1 $1.50000000 \mathrm{sec}$

TDO

क거

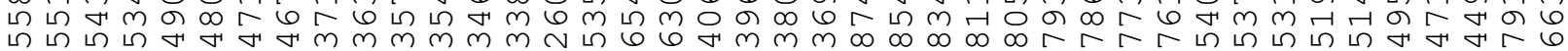

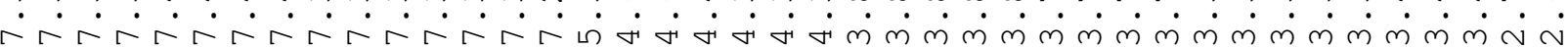

$=======$ CHANNEL fl $========$ NUC1

P1 1

13.60 use

PL1W $\quad 7.55784369 \mathrm{~W}$

$400.1326010 \mathrm{MHz}$

F2 - Processing parameters

$\begin{array}{lr}\text { SI } & 32768 \\ \text { SF } & 400.1300096 \mathrm{MHz}\end{array}$

WDW

SSB

LB

GB
PC

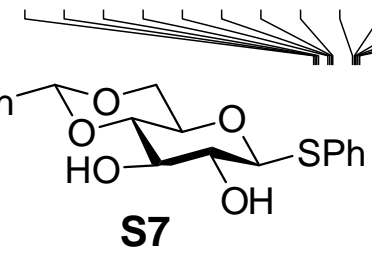

(1)

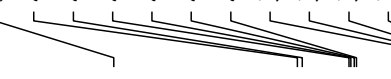

$\mathbf{S 7}$
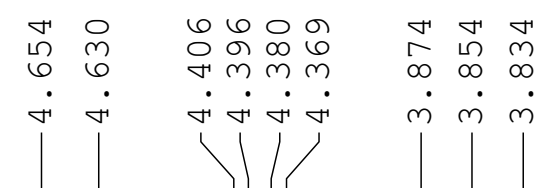

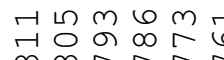

위ल댁

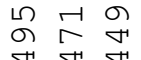

$\dot{m} \dot{m} \dot{m} \dot{m} \dot{m} \dot{m} \dot{m} \dot{m}$

$\dot{m} \dot{m} \dot{m}$

में

$m m m$
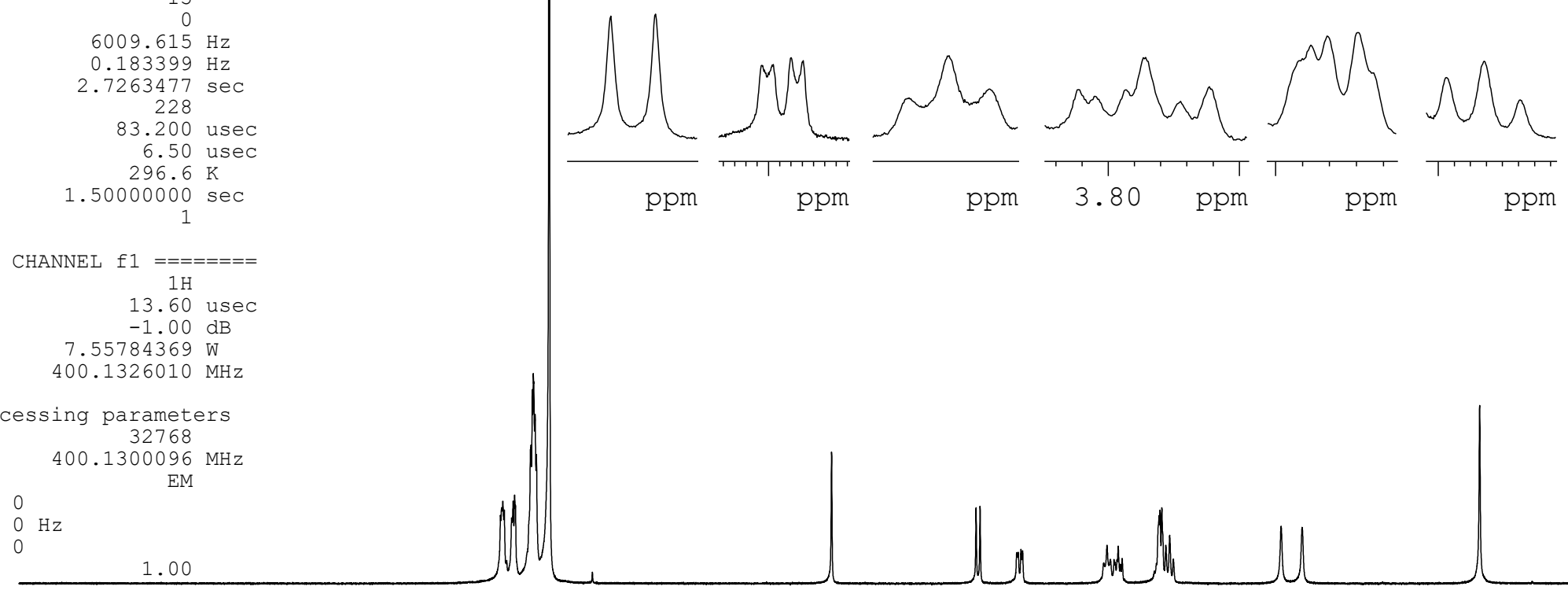

1
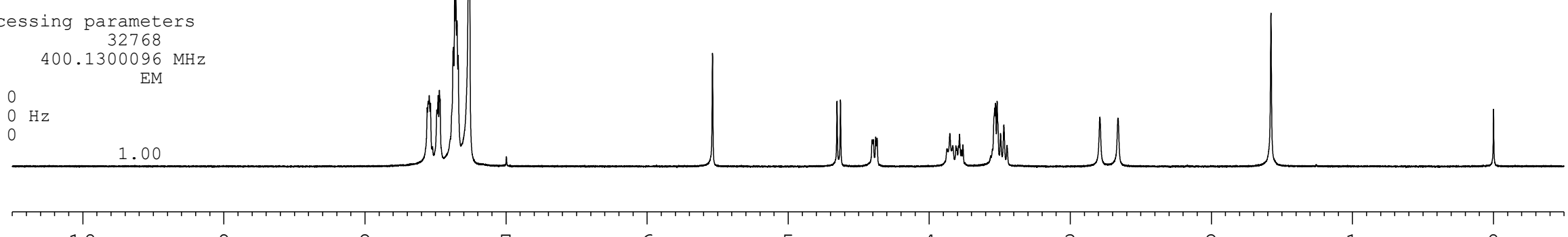

8

7

6

5

3

2

1

0 ppm

$\left|\begin{array}{c} \\ \multirow{1}{*}{} \\ 0 \\ \sim \\ 0 \\ -1\end{array}\right|$

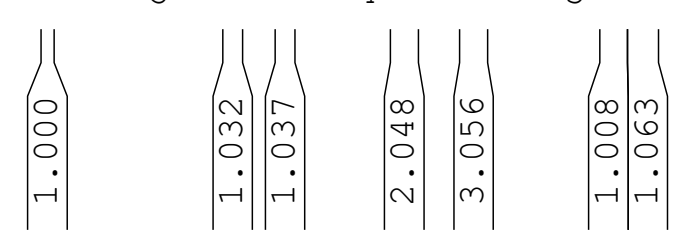




\section{${ }^{1} \mathrm{H}$ NMR $\left(400 \mathrm{MHz}, \mathrm{CDCl}_{3}\right)$ of $\mathbf{S 8 :}$}

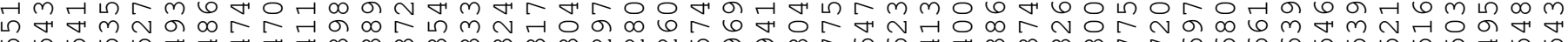
Current Data parameters?

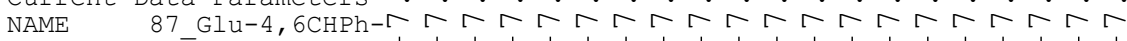
EXPNO $3 \mathrm{OBn}-\overline{2} \mathrm{OH}-\mathrm{SPh}$

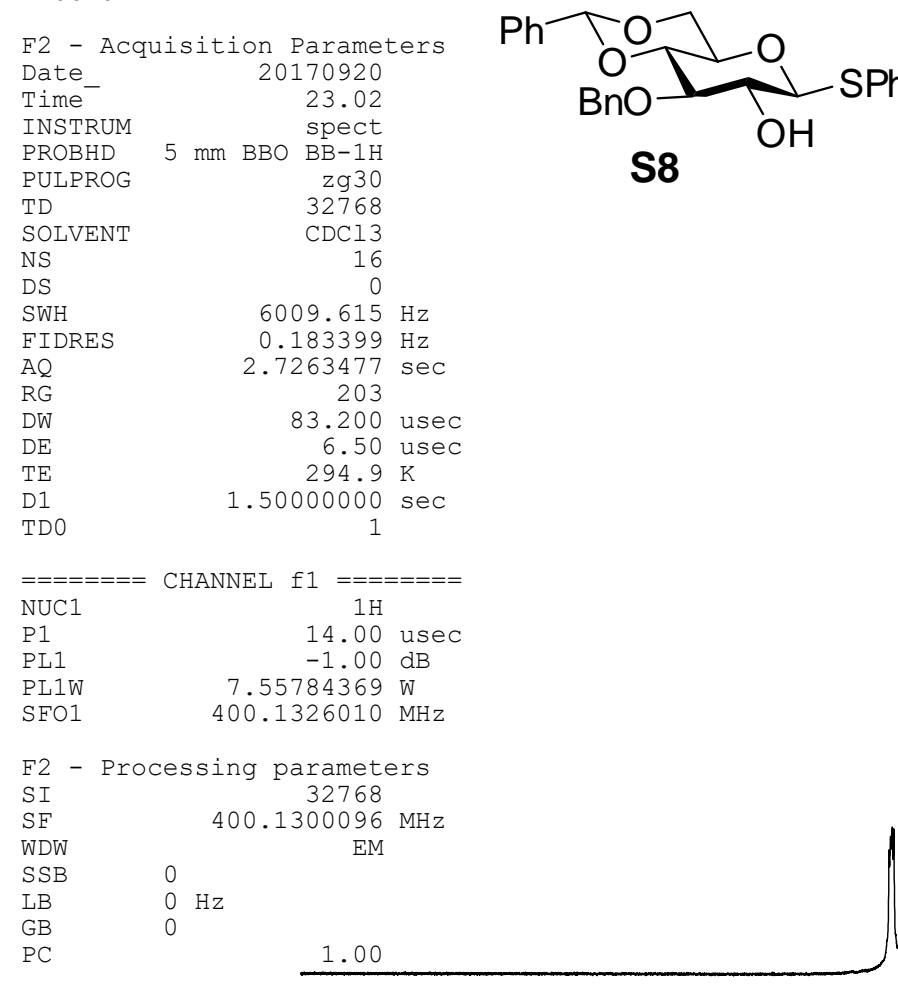

गilillin

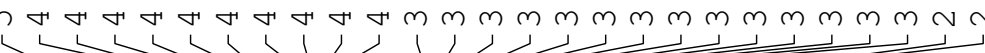
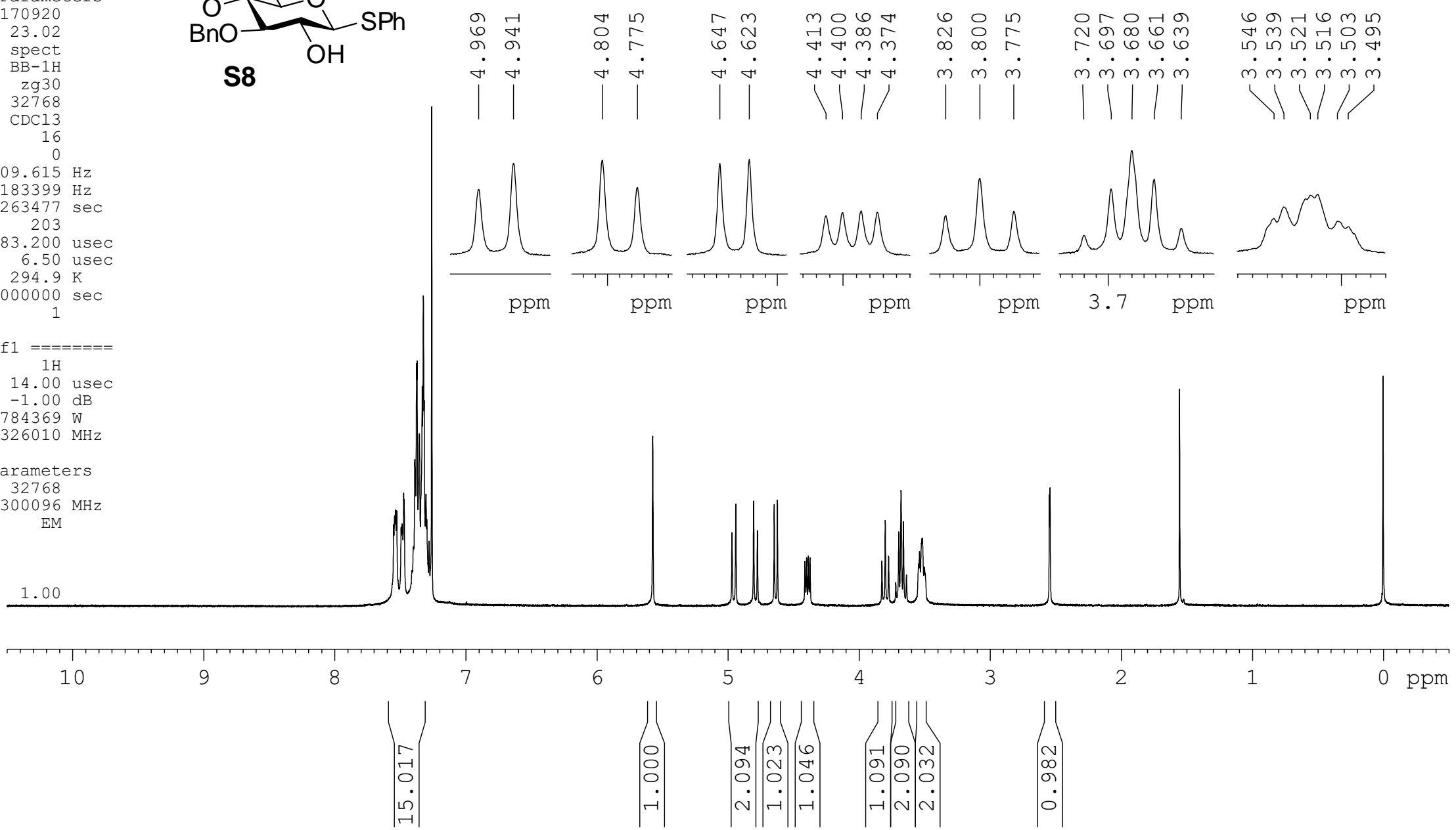
${ }^{1} \mathrm{H}$ NMR $\left(400 \mathrm{MHz}, \mathrm{CDCl}_{3}\right)$ of $\mathbf{S 9}$ :

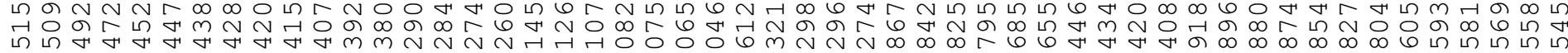

Current Data Parameters

$88-\mathrm{Glu}-4,6 \mathrm{CHPh}$
3OBn-2OBz-SPh

EXPNO

F2 - Acquisition Parameters

Date__ 20170924

Time
INSTRUM

12.55

PROBHD
PULPROG

$5 \mathrm{~mm}$ BBO $\mathrm{spect}$

PULPROG
TD

SOLVENT
NS

NS
DS

FIDRES

$\mathrm{AQ}$
$\mathrm{RG}$

RG
DW

DE

DE

TDO
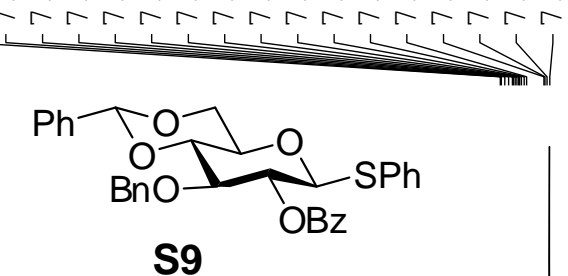

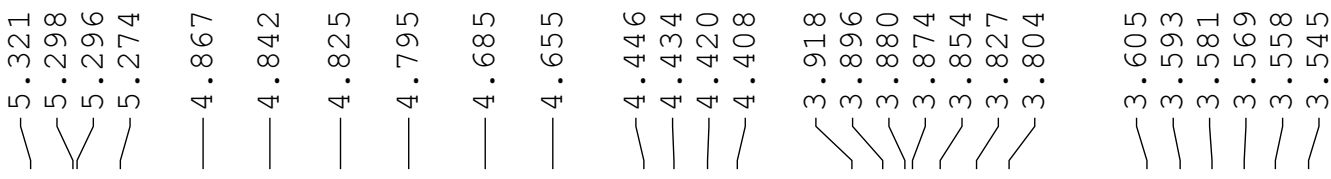

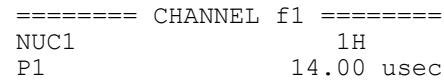

PL1 $\quad-1.00 \mathrm{~dB}$

$\begin{array}{lr}\text { PLIW } & 7.55784369 \mathrm{~W} \\ \text { SFO1 } & 400.1326010 \mathrm{MH}\end{array}$

F2 - Processing parameters

$\begin{array}{ll}\text { SI } & 32768 \\ \text { SF } & 400.1300096 \\ \mathrm{MHz}\end{array}$

WDW

$\begin{array}{ll}\mathrm{SSB} & 0 \\ \mathrm{LB} & 0 \\ \mathrm{~GB} & 0\end{array}$

PC

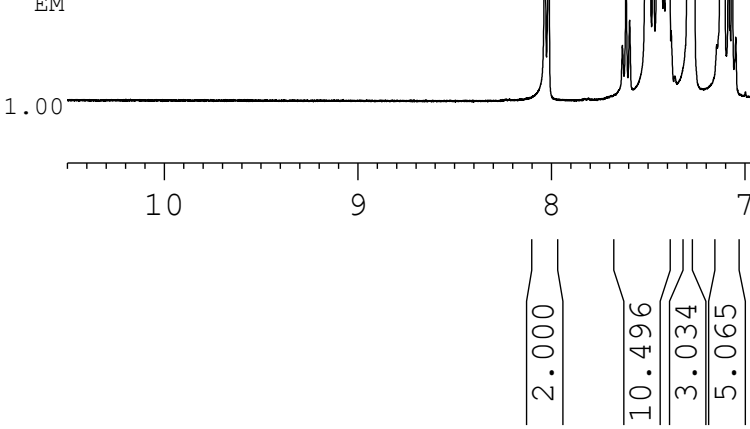

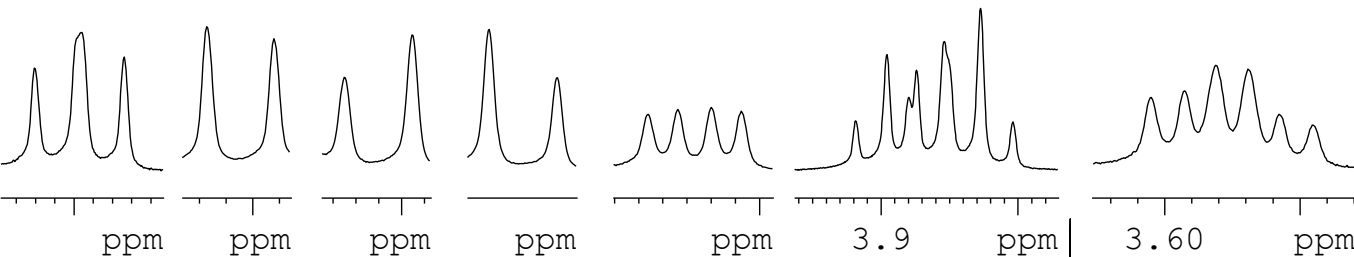

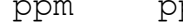

(2.

7

5

4

3

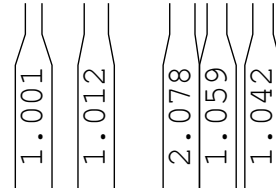

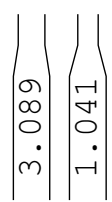




\section{${ }^{1} \mathrm{H}$ NMR (400 MHz, $\left.\mathrm{CDCl}_{3}\right)$ of $\mathbf{S 1 0}$ :}

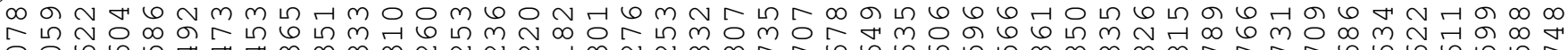

Current Data Parameters $\dot{0} \dot{0}$

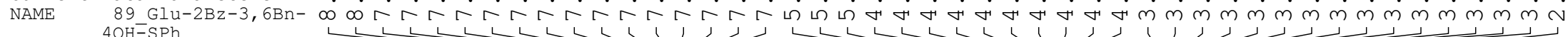
EXPNO
PROCNO

F2 - Acquisition Parameters

Date_ 2017092

Time $\quad 11.32$

INSTRUM $5 \mathrm{~mm}$ BBO $\begin{aligned} & \text { spect } \\ & \mathrm{BB}-1 \mathrm{H}\end{aligned}$

PROBHD

PULPROG

SOLVENT

NS

DS

FIDRES $\quad 0.183399 \mathrm{~Hz}$

$\mathrm{AQ} \quad 2.7263477 \mathrm{~Hz}$

$\begin{array}{lr}\text { RG } & 90.5 \\ \text { DW } & 83.200 \text { usec }\end{array}$

$\begin{array}{lr}\text { DE } & 6.50 \text { usec }\end{array}$

TE

$\begin{array}{lr}\text { D1 } & 1.50000000 \mathrm{sec} \\ \text { TD0 } & 1\end{array}$

$=======$ CHANNEL $\mathrm{fl}=======$
NUC1 $1 \mathrm{H}$

$\mathrm{P} 1$
$\mathrm{PL} 1$

PL1W
SFO1

14.00 usec

F2 - Processing parameters

SI 32768

$\begin{array}{lc}\text { SF } & 400.1300099 \mathrm{MHz} \\ \text { WDW } & \mathrm{EM}\end{array}$

$\begin{array}{ll}\text { WSW } & 0 \\ \text { LB } & 0 \\ \text { H }\end{array}$

$\begin{array}{lll}\mathrm{LB} & 0 & \mathrm{~Hz} \\ \mathrm{~GB} & 0\end{array}$
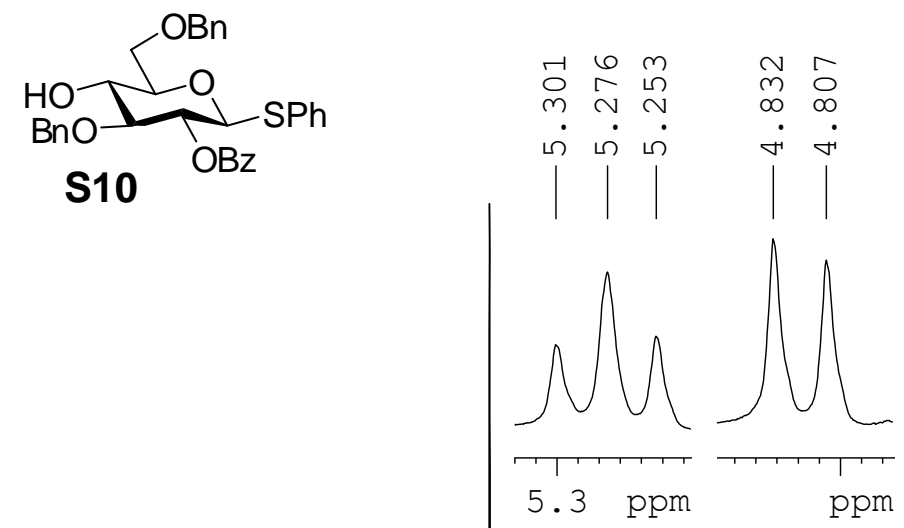

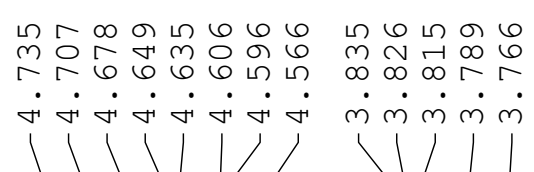

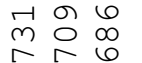

mָ

.

S10
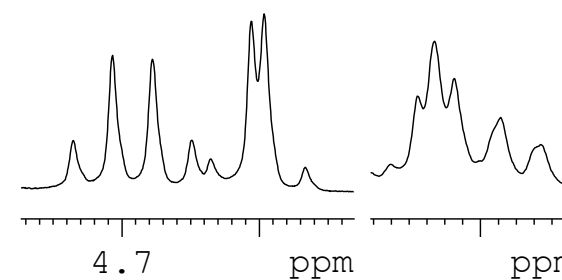

- \11
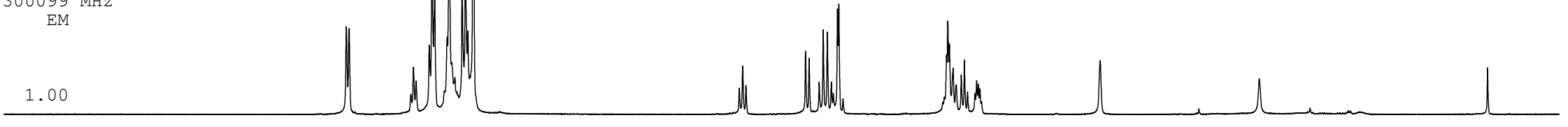

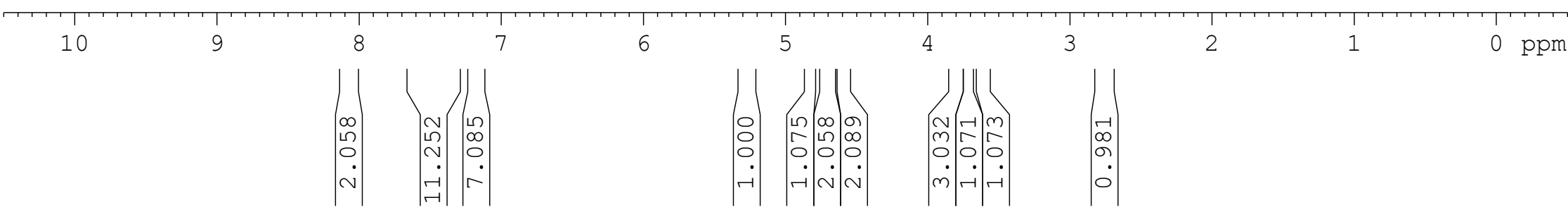




\section{${ }^{1} \mathrm{H}$ NMR (400 MHz, $\mathrm{CDCl}_{3}$ ) of $\mathbf{1 7}$ :}

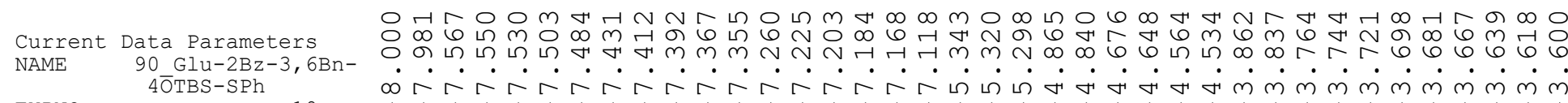

EXPNO 4OTBS-SPh 10 orrrriririm

PROCNO

F2 - Acquisition Parameters

Time-

INSTRUM

PROBHD $5 \mathrm{~mm}$ BBO $\mathrm{BB}-1 \mathrm{H}$

PULPROG

TD

NS

DS

SWH
FIDRES

$\mathrm{AQ}$

RG

$\mathrm{DW}$
$\mathrm{DE}$
$\mathrm{TE}$

TE

TDO

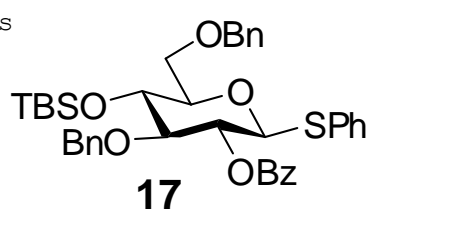
$\stackrel{m}{\sim} \stackrel{\infty}{\sim}$
$m m$ ?
i

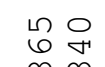

ש

ซื

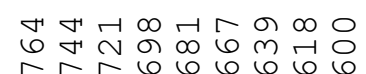

$\dot{m} \dot{m}$

$\dot{m} \dot{m} \dot{m} \dot{m} \dot{m} \dot{m} \dot{m}$

$|1|$

1) 11

16

$6009.615 \mathrm{~Hz}$

$0.183399 \mathrm{~Hz}$

83.200 usec

83.200 usec
6.50 usec

$293.7 \mathrm{~K}$
$.50000000 \mathrm{sec}$
$=====$
NUC1
$\mathrm{P} 1$
P1
PL1

PL1W
SFO1
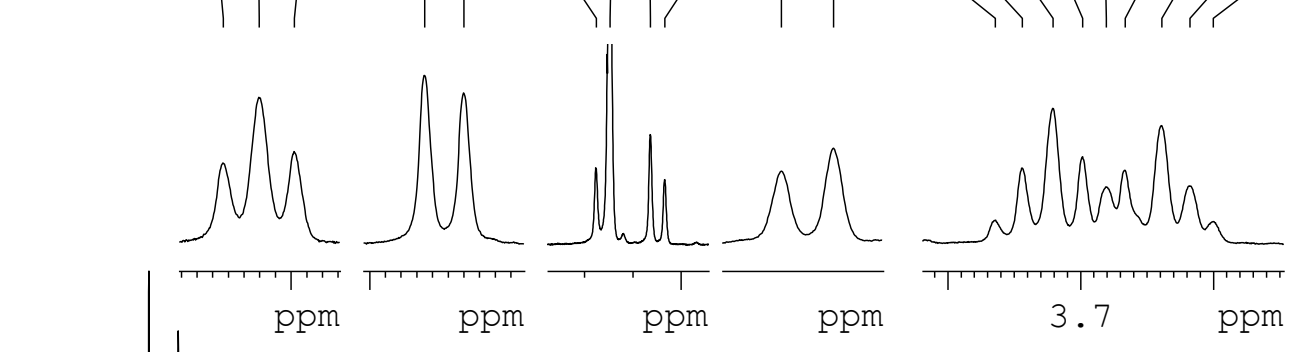

F2 - Processing parameter

$\begin{array}{lr}\text { SI } & 32768 \\ \text { SF } & 400.1300095 \\ \text { MHz }\end{array}$

WDW

SSB

LB
GB

0
0 $\mathrm{~Hz}$

1300095
EM

PC

1

1.00

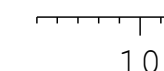

10

9

7

6

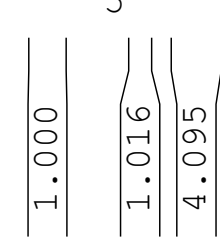

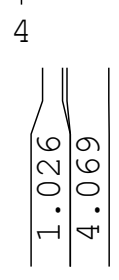

3

2

$\begin{aligned} & 1 \\ & \mid \\ & n \\ & 0 \\ & 0 \\ & 0\end{aligned} \mid$

0 ppm 
${ }^{13} \mathrm{C}$, DEPT-90 and DEPT-135 NMR (101 MHz, $\left.\mathrm{CDCl}_{3}\right)$ of 17:

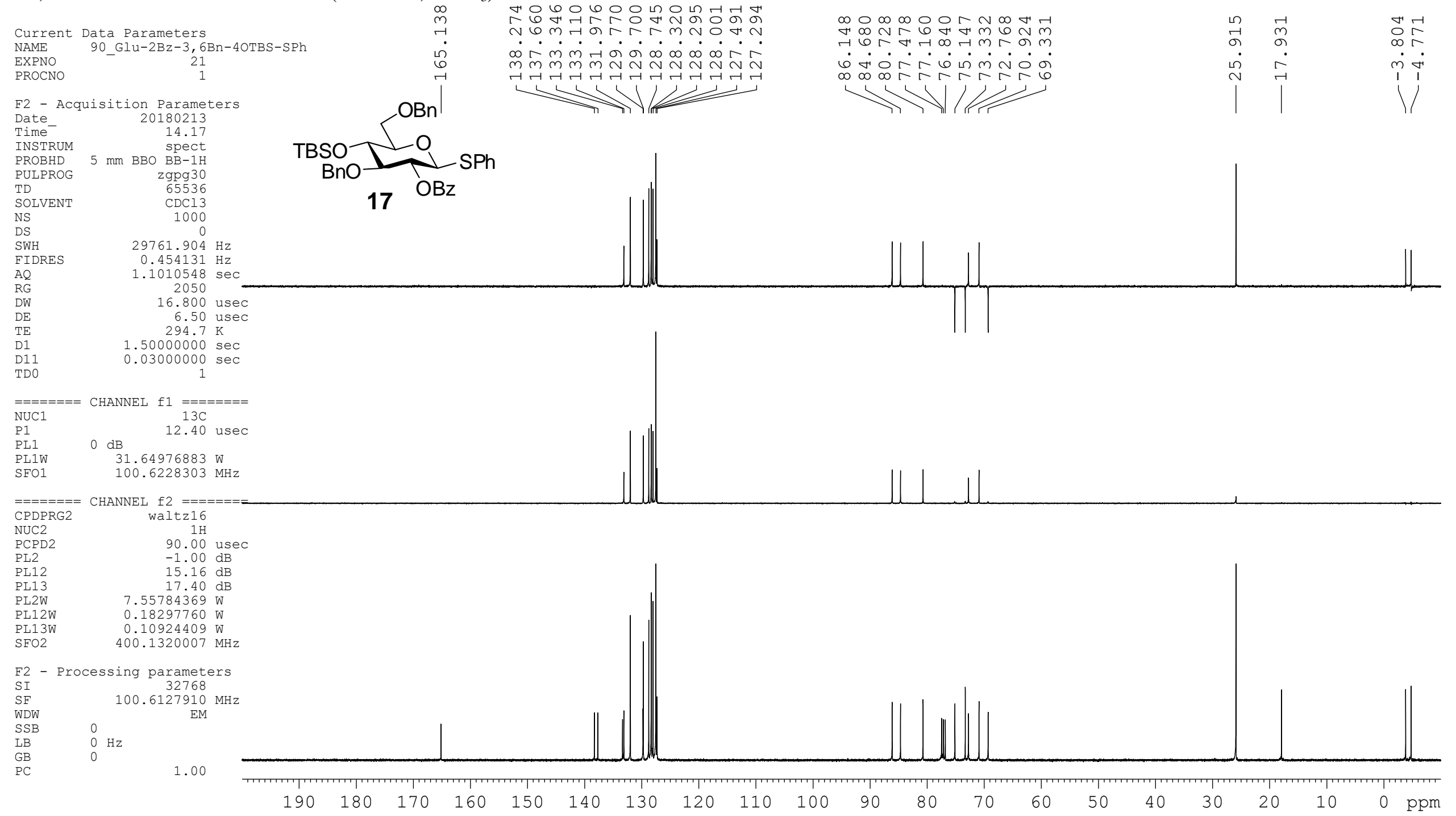


${ }^{1} \mathrm{H}$ NMR (400 MHz, $\mathrm{CDCl}_{3}$ ) of $\mathbf{S 1 2 :}$

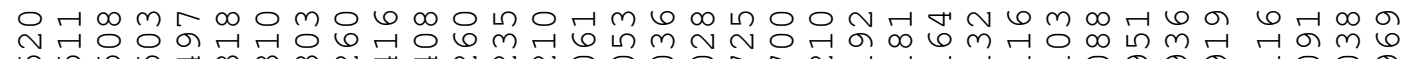

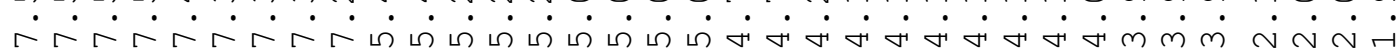

Current Data Parameters NAME 79 Gal-Ac-SPh EXPNO $\longrightarrow$ $\underset{1}{\longrightarrow}$ भमन

$\begin{array}{lr} & 1 \\ \text { F2 - Acquisition } & \text { Parameters } \\ \text { Date_ } & 20170830 \\ \text { Time } & 14.06 \\ \text { INSTRUM } & \text { spect } \\ \text { PROBHD } & \text { 5 m BBO BB-1H } \\ \text { PULPROG } & \text { zg30 } \\ \text { TD } & 32768 \\ \text { SOLVENT } & \text { CDC13 } \\ \text { NS } & 10 \\ \text { DS } & 0 \\ \text { SWH } & 6009.615 \mathrm{~Hz} \\ \text { FIDRES } & 0.183399 \mathrm{~Hz} \\ \text { AQ } & 2.7263477 \mathrm{sec} \\ \text { RG } & 128 \mathrm{u} \\ \text { DW } & 83.200 \mathrm{use} \\ \text { DE } & 6.50 \mathrm{use} \\ \text { TE } & 294.9 \mathrm{~K} \\ \text { D1 } & 1.5000000 \mathrm{sec} \\ \text { TDO } & 1 \\ & \end{array}$

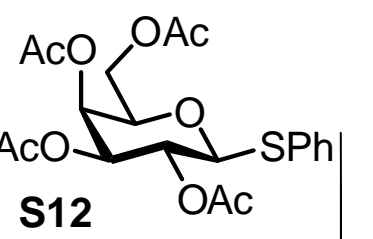

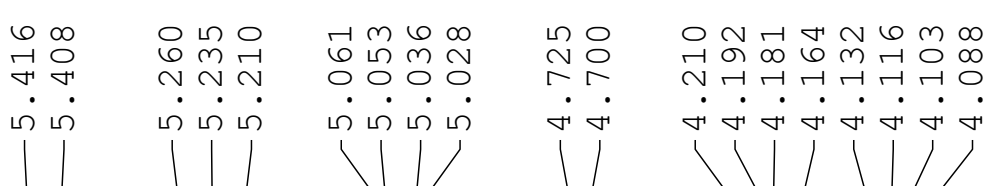

다 6

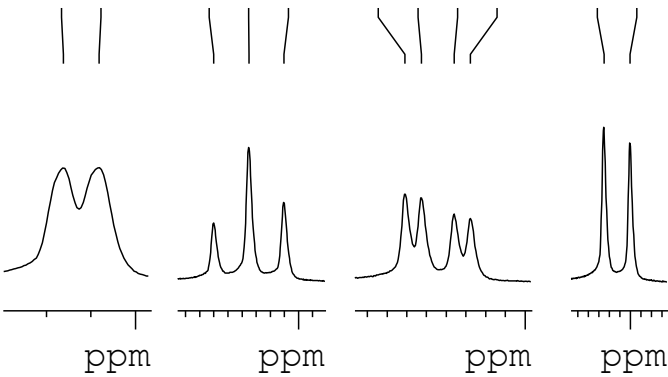

$\dot{m} \dot{m}$

$1 \mid$

$=======$ CHANNEL $\mathrm{f} 1 \mathrm{l}=======$

$1 \mathrm{H}$
.00 usec

$\begin{array}{lr}\text { P1 } & 14.00 \text { us } \\ \text { PL1 } & -1.00 \mathrm{~dB}\end{array}$

$\mathrm{PL1W}$
$\mathrm{SFO1}$

F2 - Processing parameters

SI 32768

SF $\quad 400.1300097 \mathrm{MHz}$

WDW

SSB

LB

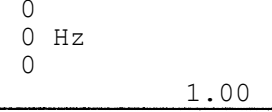

10
$1+1+1+1+1,1$

98

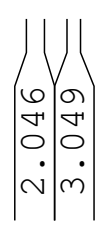

7

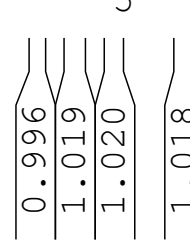

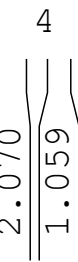

1

0 ppm 


\section{${ }^{1} \mathrm{H}$ NMR $\left(400 \mathrm{MHz}, \mathrm{CDCl}_{3}\right)$ of $\mathbf{S 1 3}$}

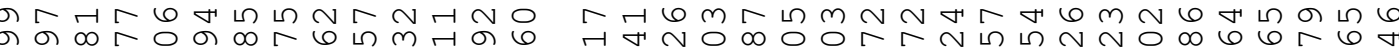
Я

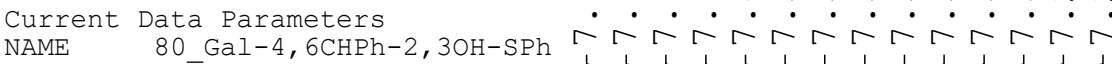

NAME

80_Gal-4,6CHPh-2, 30H-SPh

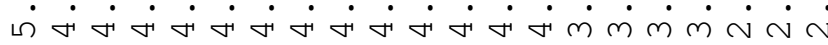

PROCNO

F2 - Acquisition Parameters

Date__ 20170903

18.16

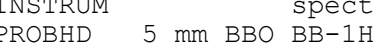

PROBHD

PULPROG

SOLVENT

NS

DS

FIDRES

$\mathrm{AQ}$

RG

DW

DE

D1

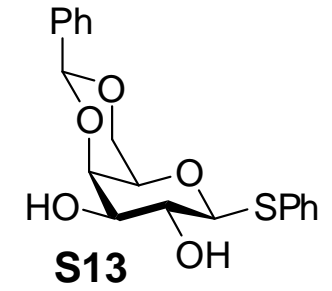

$====$

$\mathrm{zg} 30$
32768
$\mathrm{CDCl}$

$\mathrm{CDC} 13$
16

$6009.615 \mathrm{~Hz}$ $0.183399 \mathrm{~Hz}$

203

203
83.200 usec

6.50 usec

$1.50000000 \mathrm{sec}$

CHANNEL $\mathrm{f}$

$========$

PL1
PL1W

PL1W
SFO1

14.00 usec

$-1.00 \mathrm{~dB}$

$7.55784369 \mathrm{~W}$

F2 - Processing parameters

SI $\quad 32768$

$097 \mathrm{MHz}$

$\begin{array}{ll}\text { SSB } & 0 \\ \text { LB } & 0\end{array}$

GB

GB
PC

$0 \mathrm{~Hz}$

(1)

.00
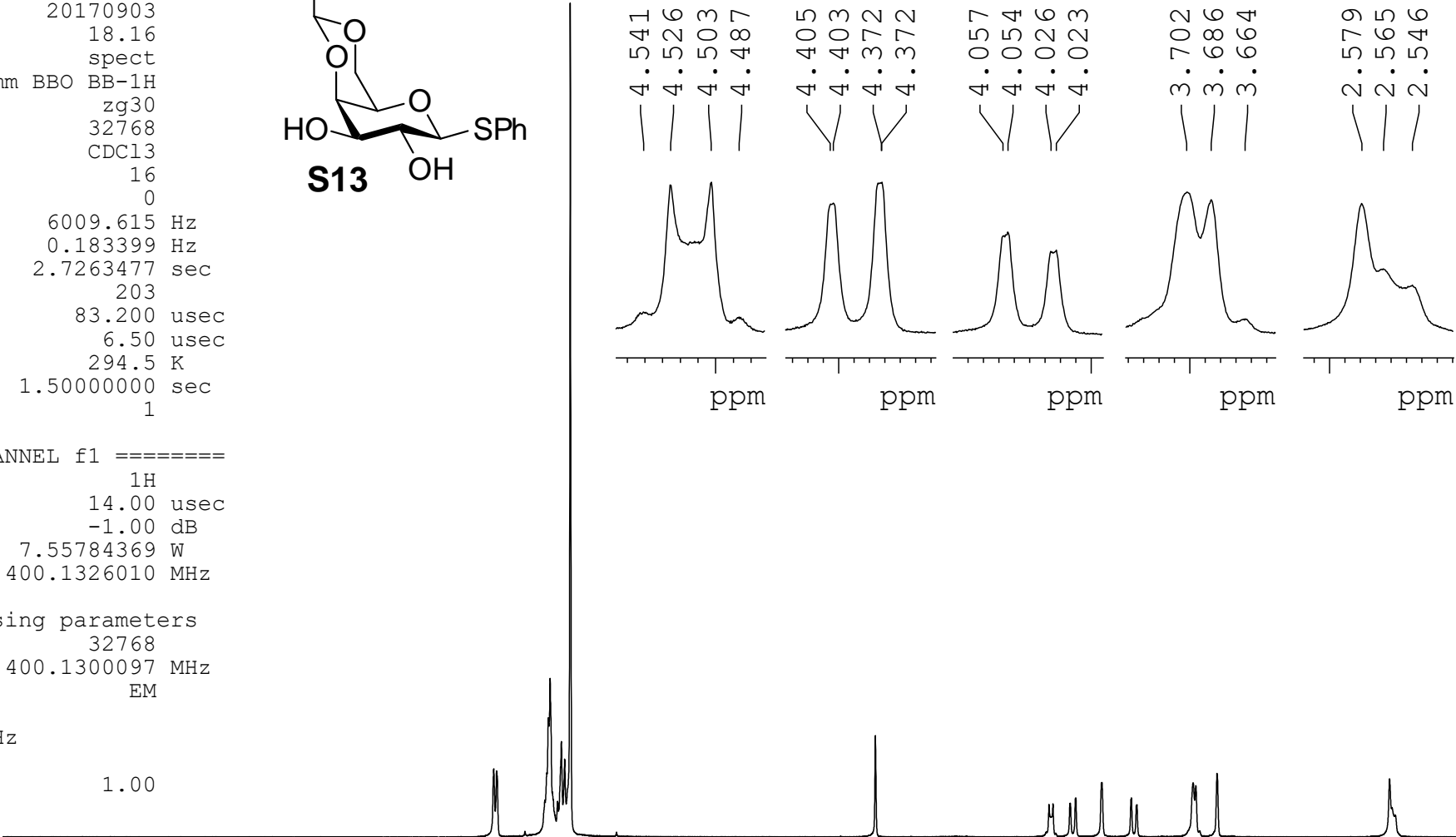

MLLIII

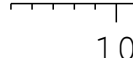

9

8

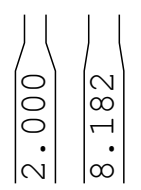

6

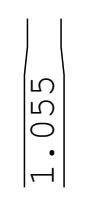

5

4

3

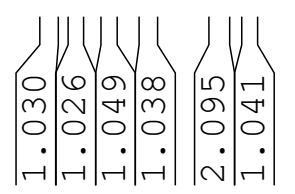

2

1

0 ppm

$\left|\begin{array}{c} \\ 0 \\ 0 \\ 0 \\ 0 \\ \dot{N}\end{array}\right|$ 


\section{${ }^{1} \mathrm{H}$ NMR (400 MHz, $\mathrm{CDCl}_{3}$ ) of $\mathbf{S 1 4 :}$}

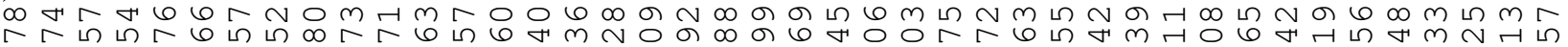

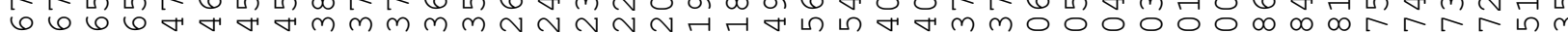

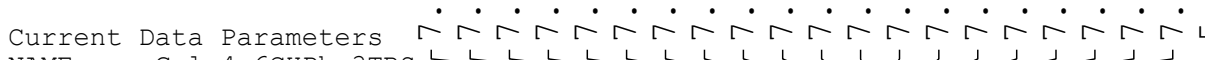
NAME Gal-4, 6CHPh-3TBS-

EXPNO
PROCNO $2 \mathrm{OH}-\mathrm{SPh}$

F2 - Acquisition Parameters

Time ${ }^{-}$

TROBR

PROBHD

20170903

$5 \mathrm{~mm} \mathrm{BBO} \mathrm{BB}-1 \mathrm{H}$

SOLVENT

NS

SWH

FIDRES

$\mathrm{AQ}$

DW

$\mathrm{DE}$

D1

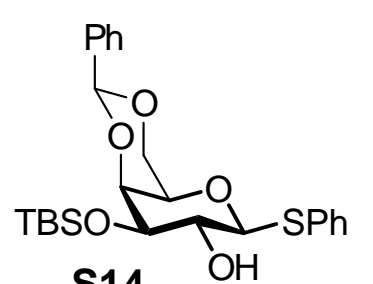

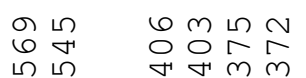

.

tr $m$ m

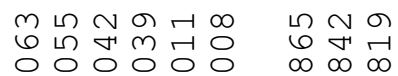

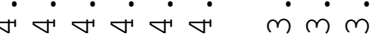

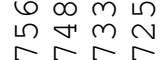

$\mathrm{zg} 30$
32768

$\mathrm{CDCl} 3$

$\mathrm{S} 14$

$6009.615 \mathrm{~Hz}$

$0.183399 \mathrm{~Hz}$

$2.7263477 \mathrm{sec}$

83.200 usec

6.50 use

$1.50000000 \mathrm{sec}$

TDO

$\begin{array}{lr}=======\text { CHANNEL } \mathrm{fl}======== \\ \text { NUC1 } & 1 \mathrm{H} \\ \text { P1 } & 13.60 \mathrm{useC} \\ \text { PL1 } & -1.00 \mathrm{~dB} \\ \text { PL1W } & 7.55784369 \mathrm{~W} \\ \text { SFO1 } & 400.1326010 \mathrm{MHz}\end{array}$

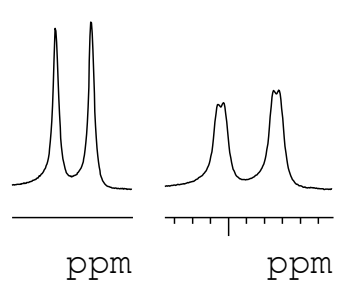

$\dot{\nabla} \dot{\nabla} \dot{\gamma} \dot{\gamma}$

F2 - Processing

SI - Processing parameters

$\begin{array}{lr}\text { SI } & 32768 \\ \text { SF } & 400.1300087 \mathrm{MHz}\end{array}$

$\begin{array}{lr}\text { SF } & 400.1300087 \\ \text { WDW } & \text { EM }\end{array}$

WDW $\quad 0$

$\begin{array}{lll}\text { LB } & 0 & \mathrm{~Hz} \\ \text { GB } & 0 & \end{array}$

$\mathrm{PC}$

${ }_{0} \mathrm{~Hz}$

1.00
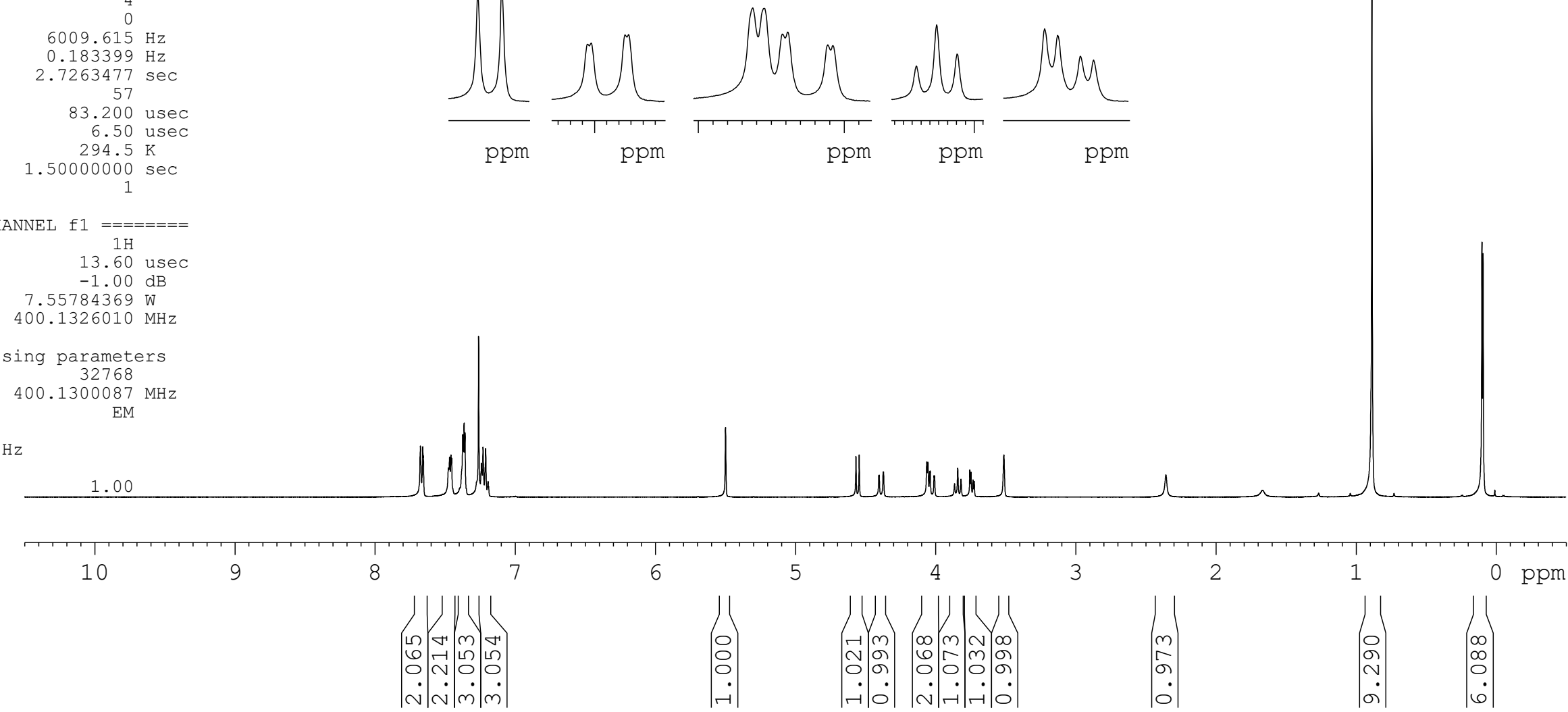


\section{${ }^{1} \mathrm{H}$ NMR (400 MHz, $\mathrm{CDCl}_{3}$ ) of $\mathbf{S 1 5}$ :}

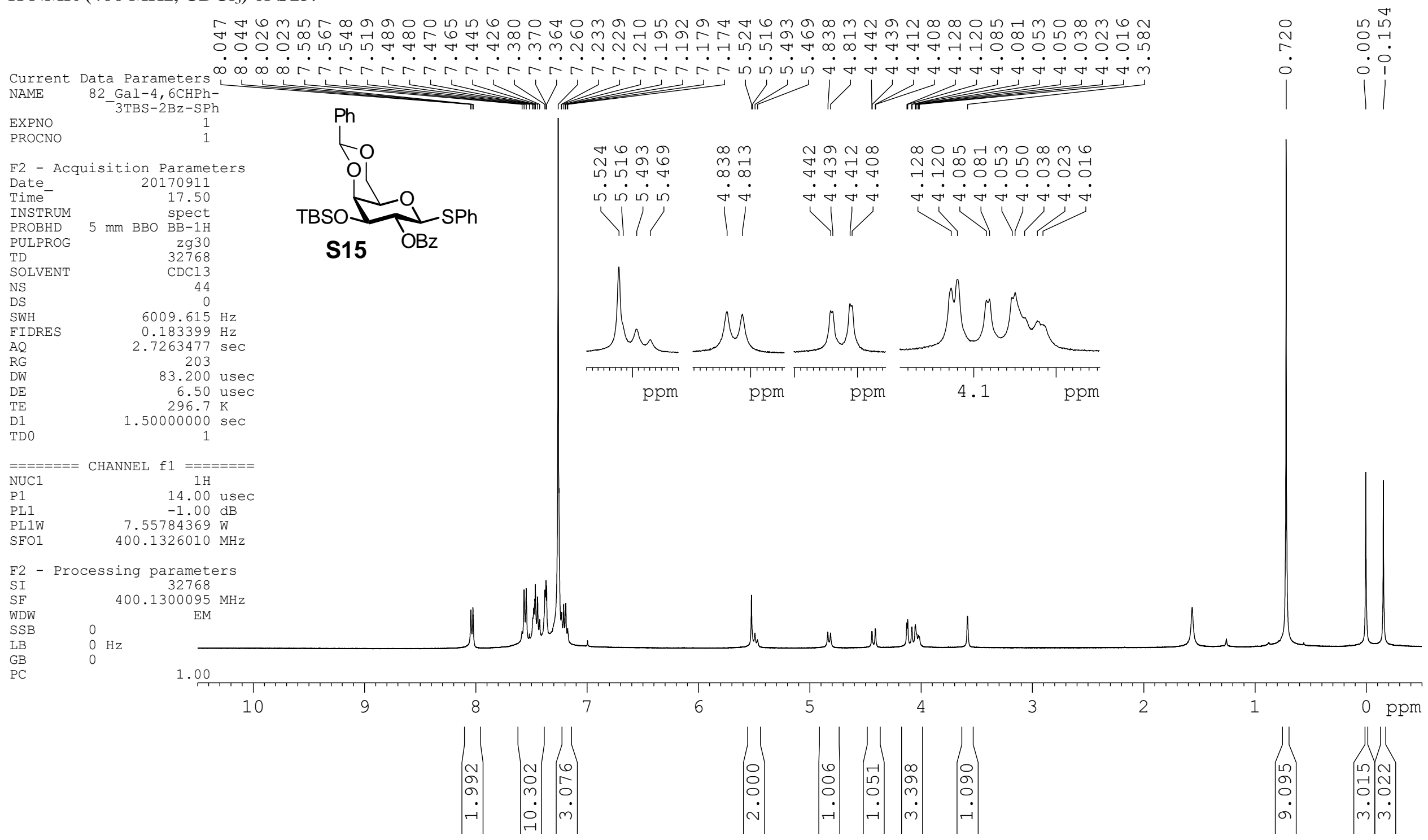


${ }^{13} \mathrm{C}$, DEPT-90 and DEPT-135 NMR (101 MHz, $\mathrm{CDCl}_{3}$ ) of $\mathbf{S 1 5}$ :

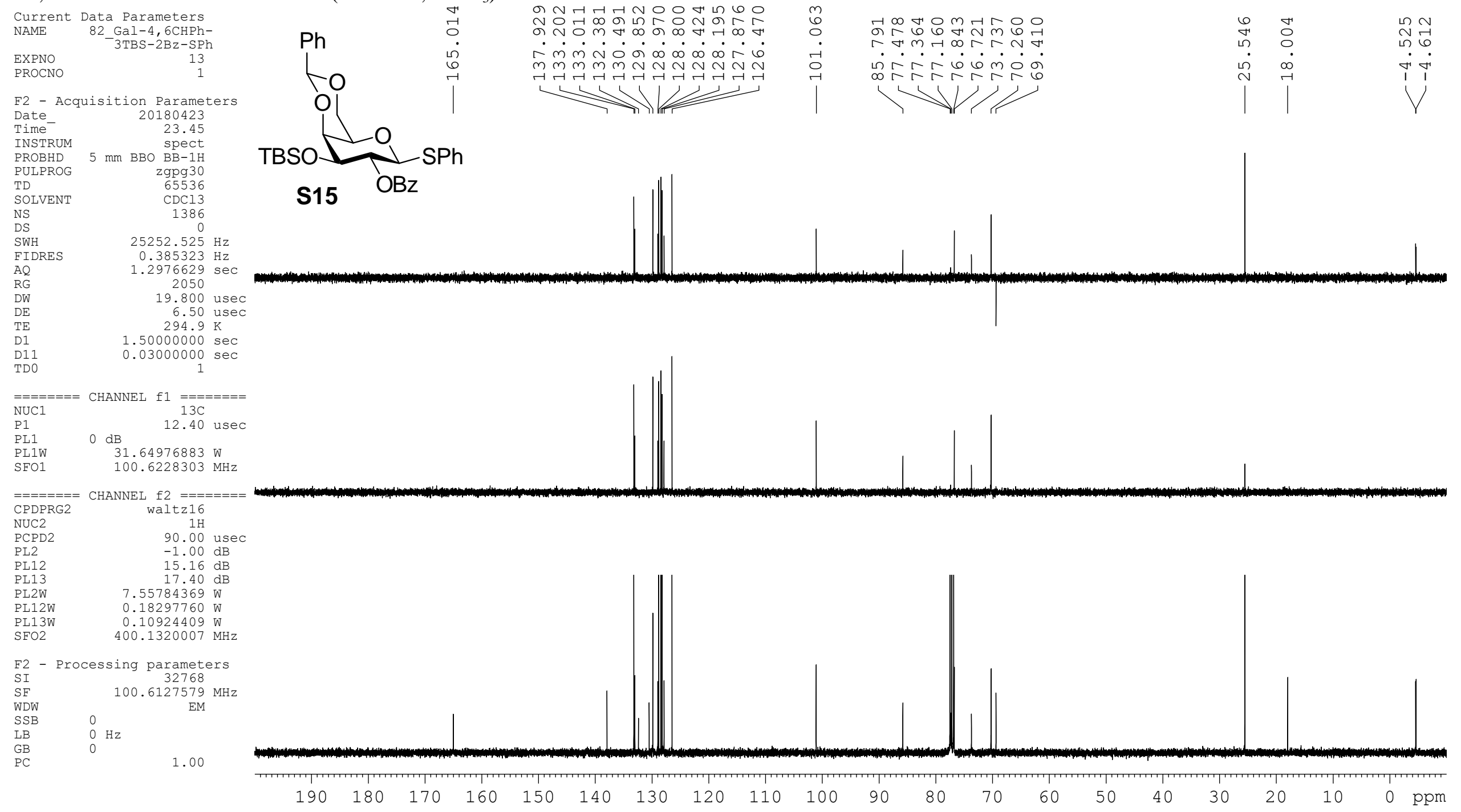




\section{${ }^{1} \mathrm{H}$ NMR (400 MHz, $\mathrm{CDCl}_{3}$ ) of 21:}

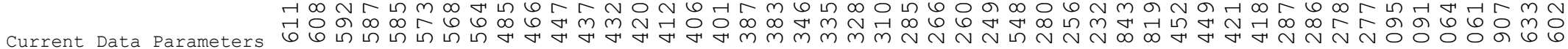
EXPNO PROCNO

$\begin{array}{lr}\text { F2 - Acquisition Parameters } \\ \text { Date- } \\ \text { Da170924 } \\ \text { Time } & 12.46 \\ \text { INSTRUM } & \text { spect } \\ \text { PROBHD } & 5 \mathrm{~mm} \text { BBO BB-1H } \\ \text { PULPROG } & \text { zg30 } \\ \text { TD } & 32768 \\ \text { SOLVENT } & \text { CDC13 } \\ \text { NS } & 28 \\ \text { DS } & 0 \\ \text { SWH } & 6009.615 \mathrm{~Hz} \\ \text { FIDRES } & 0.183399 \mathrm{~Hz} \\ \text { AQ } & 2.7263477 \mathrm{sec} \\ \text { RG } & 203 \mathrm{sec} \\ \text { DW } & 83.200 \mathrm{usec} \\ \text { DE } & 6.50 \mathrm{usec} \\ \text { TE } & 294.7 \mathrm{~K} \\ \text { D1 } & 1.5000000 \mathrm{sec} \\ \text { TDO } & 1\end{array}$

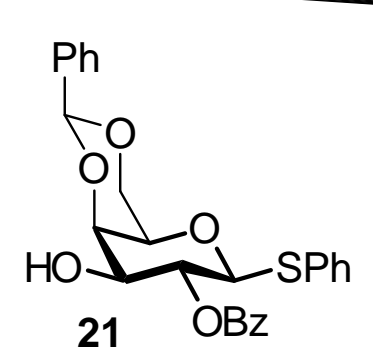

$=======$ CHANNEL f 1 l $========$

NUC1

P1

PL1 14.00 usec

PL1W $\quad 7.55784369 \mathrm{~W}$

F2 - Processing parameters

$\begin{array}{ll}\text { SI } & 32768 \\ \text { SF } & 400.1300096 \\ \text { MHz }\end{array}$

$\begin{array}{r}\text { EM } \\ \hline\end{array}$

$\begin{array}{lll}\mathrm{SSB} & 0 \\ \mathrm{LB} & 0 & \mathrm{~Hz}\end{array}$

$\mathrm{GB}$
$\mathrm{PC}$

1.00

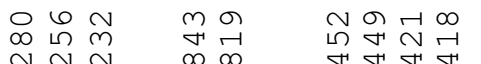

$\cdot \cdot$

เก เก เ

$1 \mid$

में

$\dot{0}$

में वं।

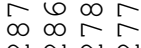

मंनं नं

बून नु

$\cdot . \cdot$.

11

$\begin{array}{llll}\text { ppm ppm } & \text { ppm } & \text { ppm } 4.1 \text { ppm }\end{array}$
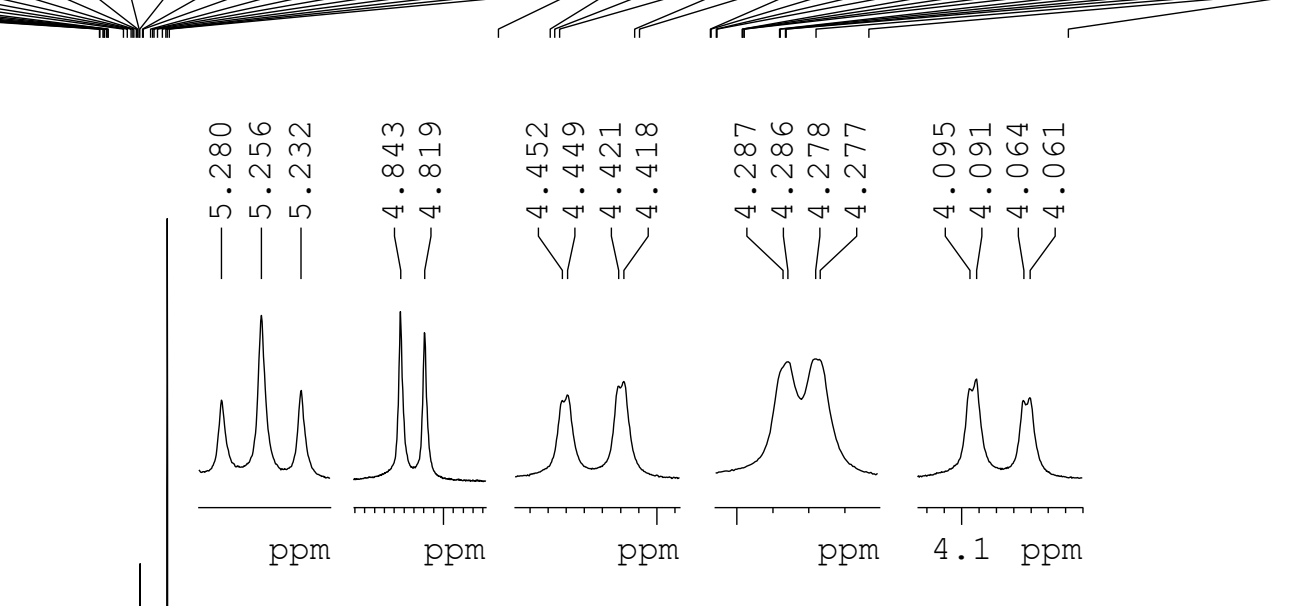

10

9

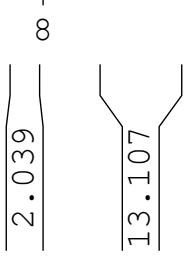

6

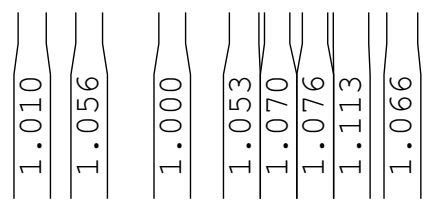

3

2

1

0 ppm 


\section{${ }^{1} \mathrm{H}$ NMR $\left(400 \mathrm{MHz}, \mathrm{CDCl}_{3}\right.$ ) of $\mathbf{S 1 6}$ :}

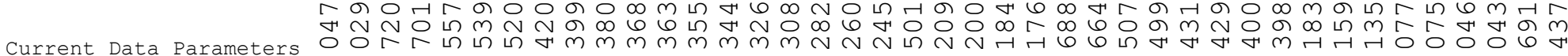

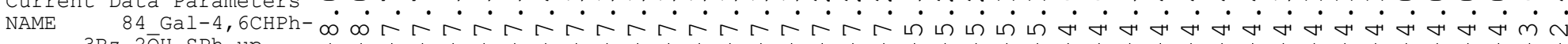

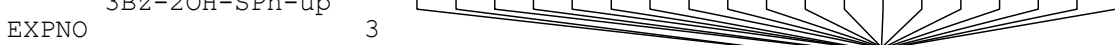

\section{EROCNO}

F2 - Acquisition Parameters

Date_ 20171103

INSTRUM

PROBHD $5 \mathrm{~mm}$ BBO BB-1H

$\begin{array}{lr}\text { PULPROG } & \text { zg30 } \\ \text { TD } & 32768\end{array}$

SOLVENT

NS

DS

FIDRES

$\mathrm{AQ}$
$\mathrm{RG}$

RG

$\mathrm{DE}$

D1

TDO

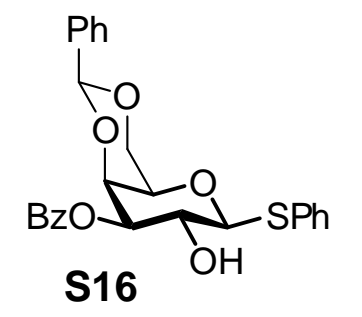
○○
ำ
ம்
111

$\longrightarrow$

1

$========$ CHANNEL fl

NUC1

P1

$7.55784369 \mathrm{~dB}$

SFO1 $400.1326010 \mathrm{MHz}$

F2 - Processing parameters

SF $\quad 400.1300097 \mathrm{MHz}$

WDW

$\begin{array}{ll}\text { SSB } & 0 \\ \text { LB } & 0 \\ \text { Hz }\end{array}$

GB

$\mathrm{Hz}$

1.00

1.00

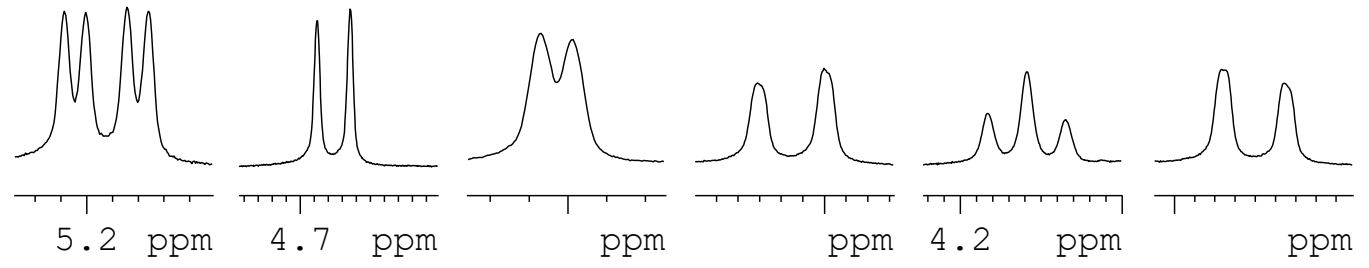

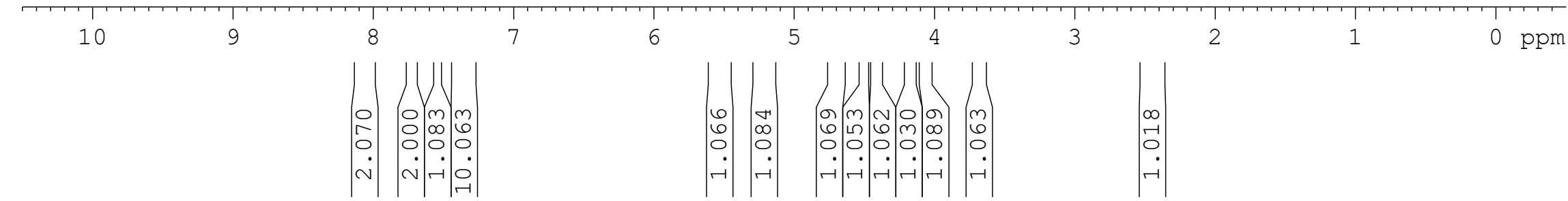


${ }^{1} \mathrm{H}$ NMR (400 MHz, $\left.\mathrm{CDCl}_{3}\right)$ of $\mathbf{S 1 8}$ :

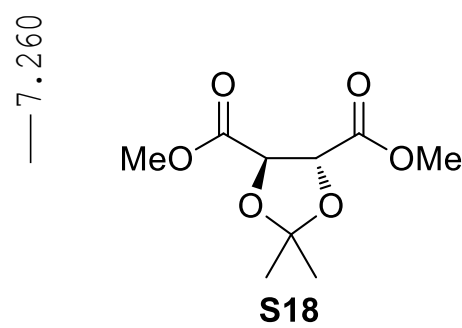

$\begin{array}{lr}\text { Current Data } & \text { Parameters } \\ \text { NAME } & 242 \\ \text { EXPNO } & 1 \\ \text { PROCNO } & 1\end{array}$

F2 - Acquisition Parameters

$\begin{array}{lr}\text { Date_- } & 20160813 \\ \text { Time } & 18.59\end{array}$

INSTRUM

PROBHD

PULPROG

TD

NS

DS

FIDRES

$\mathrm{AQ}$
$\mathrm{RG}$

RG

DW

TE

D1

TDO

$\mathrm{mm}$ BBO $\mathrm{BB}-1 \mathrm{H}$

$\mathrm{BB}-1 \mathrm{H}$
$\mathrm{zg} 30$

32768

(DC13

$6009.615 \mathrm{~Hz}$

$0.183399 \mathrm{~Hz}$

$2.7263477 \mathrm{sec}$

83.200 use

6.50 usec

$296.5 \mathrm{~K}$
$1.50000000 \mathrm{sec}$

$======$ CHANNEL f $1=======$

$\mathrm{NUC1}$

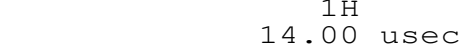

PLIW

SFO1 400.1326010 MHz

F2 - 1 100cessing porameters 8

$\begin{array}{lr}\text { SI } & 32768 \\ \text { SF } & 400.1300098 \mathrm{MHz}\end{array}$

$S S B$

$\begin{array}{lll}0 & \\ 0 & \mathrm{~Hz} \\ 0 & \end{array}$

GB

1.00

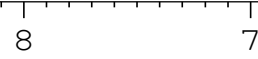

6

5

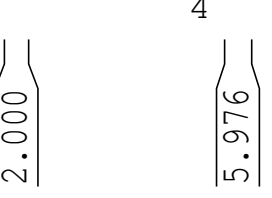

2

1

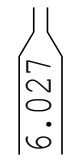


${ }^{1} \mathrm{H}$ NMR $\left(400 \mathrm{MHz}, \mathrm{CDCl}_{3}\right)$ of $\mathbf{S 1 9}$ :

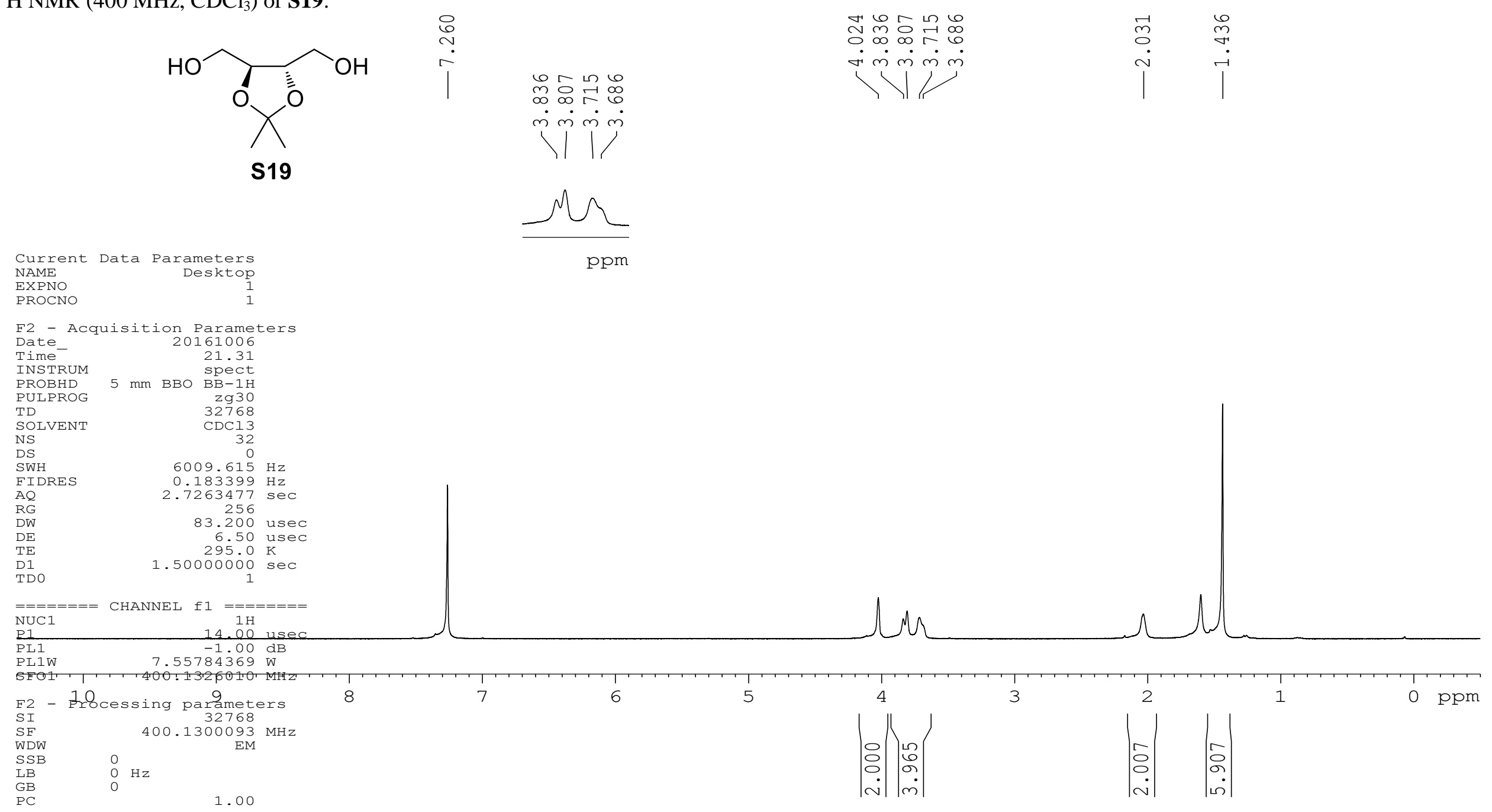


${ }^{1} \mathrm{H}$ NMR (400 MHz, $\mathrm{CDCl}_{3}$ ) of $\mathbf{S 2 0}$ :

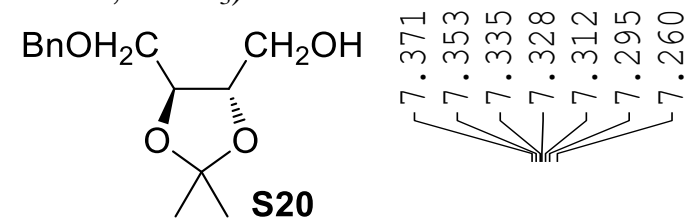

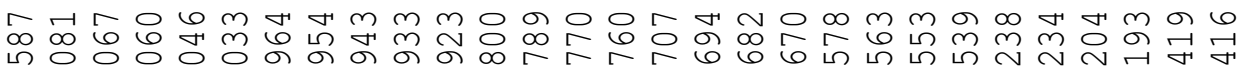

Current vaca raramecers

$$
\text { EXPNO }
$$

F2 - Acquisition Parame

Date 2016091 s

Time-

INSTRUM

PROBHD

PULPROG

TD

NS

DS

SWH

AQ

RG

$\mathrm{DE}$

TE

TDO

$=======$ CHANNEL f $1==$

NUC1

$\mathrm{P} 1$

PL1

PLIW

spect

. $\mathrm{mm}$ BBO $\mathrm{BB}-1$

$\mathrm{zg} 36$
$3276\}$

$\mathrm{CDCl}$

6009.61

0.18339
2.726347

83.201

83.5(

1.5000000

CHANNEL $\mathrm{f} 1==$

$-1.0$

7.55784365
400.1326016

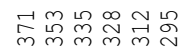

1)

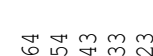

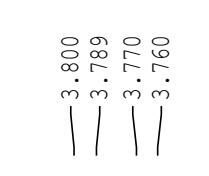

$\mid$ |l|
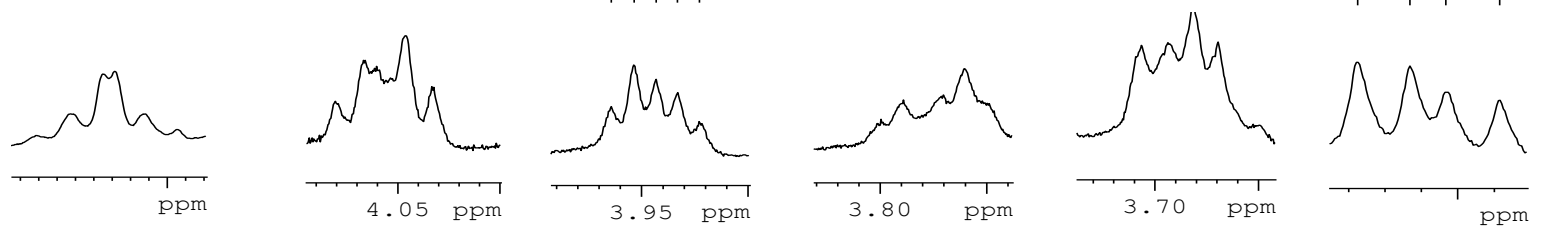

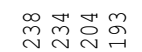

Vijij
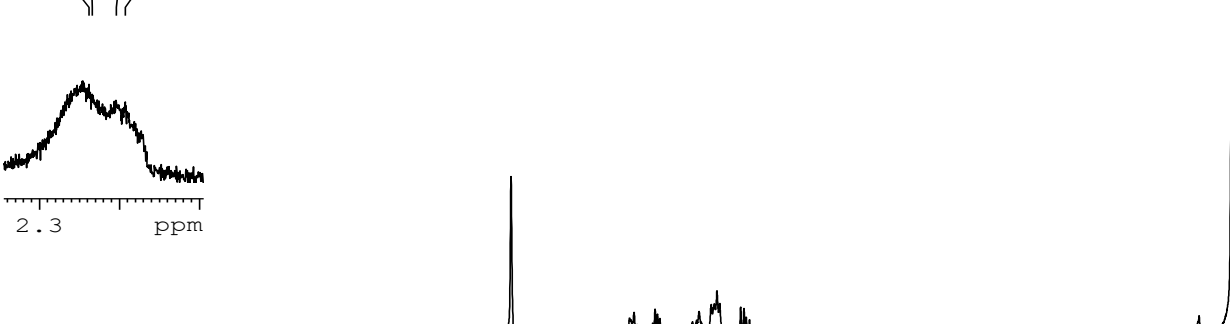

1.1 1

9

8

$\left|\begin{array}{l}\infty \\ \infty \\ 0 \\ 6 \\ \vdots \\ \llcorner\end{array}\right|$

6

5

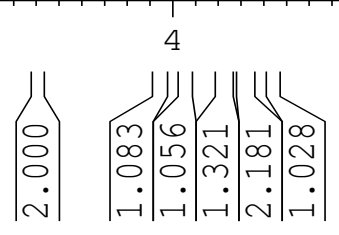

3

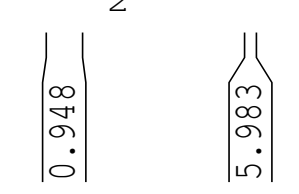

o ppm 
${ }^{1} \mathrm{H}$ NMR (400 MHz, $\mathrm{CDCl}_{3}$ ) of $\mathbf{S 2 1}$ :

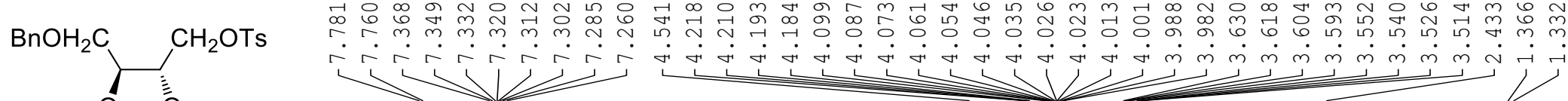

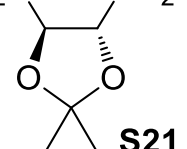

Current Data Parameters NAME

PXPNO

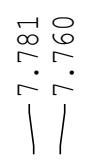

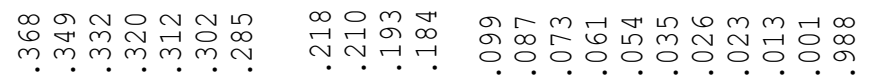

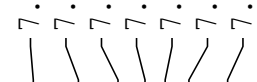

$\mid$

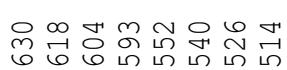

$\dot{m} \dot{m} \dot{m} \dot{m} \dot{m} \dot{m}$

F2 - Acquisition Parameters

Date 20160813

Time-

INSTRUM

PROBHD

PULPROG

spect

spect
$\mathrm{BB}-1 \mathrm{H}$

TD

NS

DS

SWH
FIDRES

$A Q$
AQ

RG

DW

DE

D1
TD0

$\mathrm{zg} 30$
32768

$\mathrm{CDCl} 13$

13
16
0

$6009.615 \mathrm{~Hz}$ $2.7263477 \mathrm{sec}$ 83.200

83.200 usec $296.5 \mathrm{~K}$

$1.50000000 \mathrm{sec}$

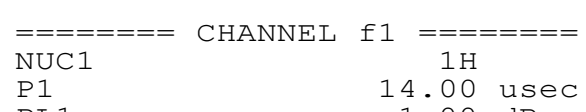

PL1

PLI 14.00 usec
$-1.00 \mathrm{~dB}$

F2 - Prdcessing parameters

$\begin{array}{lr}\text { SI } & 32768 \\ \text { SF } & 400.1300096 \mathrm{MHz}\end{array}$

SF
WDW

WDW

LB

GB

PC

0
0
0

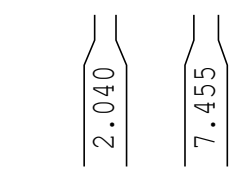
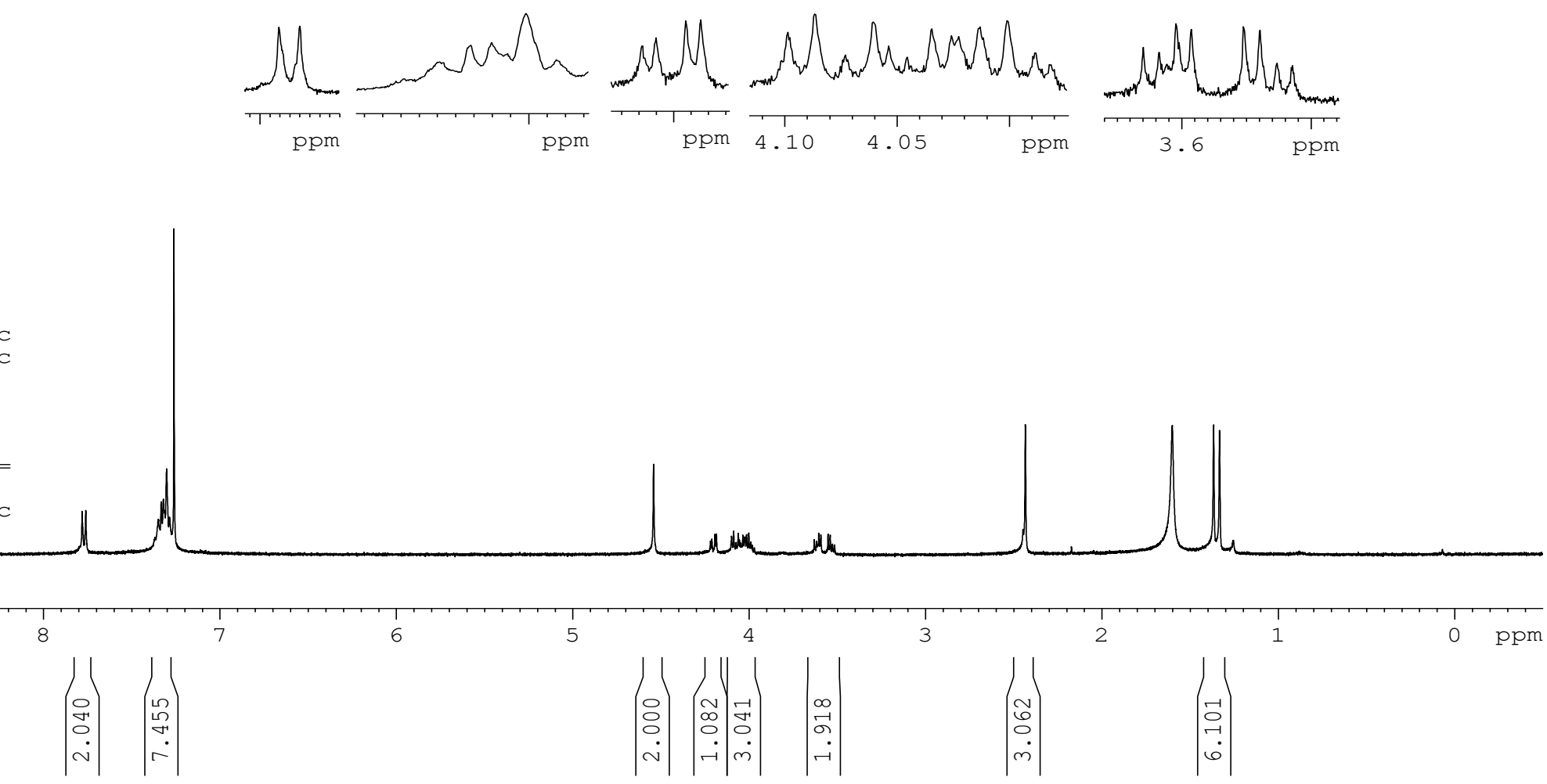
${ }^{1} \mathrm{H}$ NMR (400 MHz, $\mathrm{CDCl}_{3}$ ) of $\mathbf{S 2 2}$ :

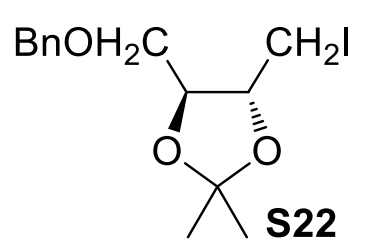

Current Data Parameters NAME

PROCNO

F2 - Acquisition Parameters Date_ 20160919

Time

INSTRUM

PUI PROG

TD

$\mathrm{SO}$

NS

SWH

AQ
RG
DW

$\mathrm{DW}$

$\mathrm{DE}$

$\mathrm{D} 1$

TDO

NUC1

P1

$\frac{\text { PLI }}{\text { PLIW }}$

SFO1

F2 -1Processing arameters 8

SI $\quad 32768$

$\mathrm{SF}$

WDW

SSB

GB

PC
$400.1300099 \mathrm{MHz}$

$\begin{array}{ll}0 & \\ 0 & H z \\ 0 & \end{array}$

1.00
$6009.615 \mathrm{~Hz}$

$2.7263477 \mathrm{sec}$

83.200 usec

6.50 use

$1.50000000 \mathrm{sec}$

CHANNEL $\mathrm{I} 1=======$ 4.00 usec $-1.00$

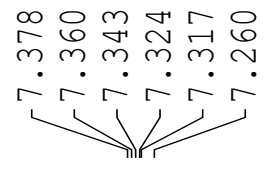

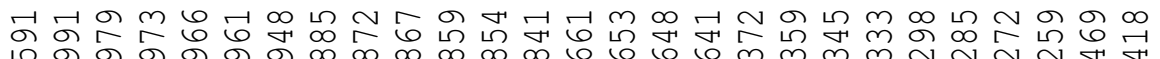
m m

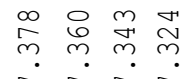

$\dot{m} \dot{m} \dot{m} \dot{m} \dot{m}$

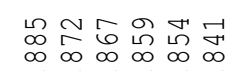

$\dot{m} \dot{m} \dot{m} \dot{m}$

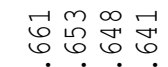

$\dot{m} \dot{m} \dot{n}$

|
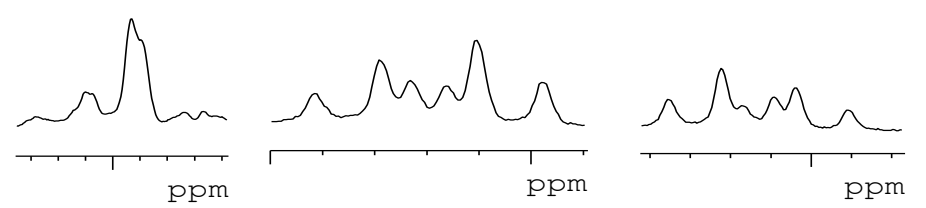

$M_{\text {ppm }}^{M}$

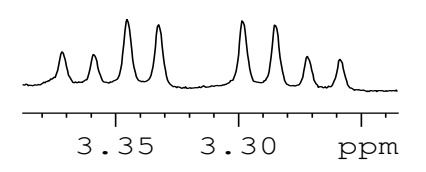

c

thel the
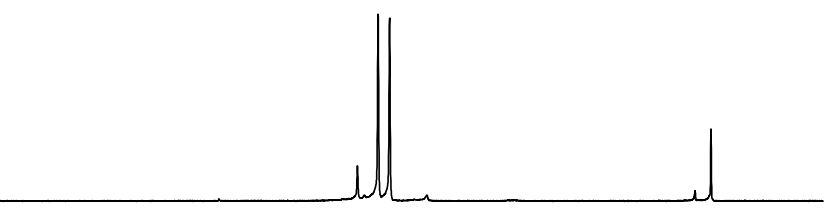

3

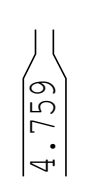

6

5

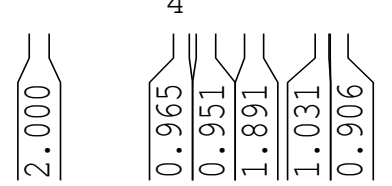

o ppm

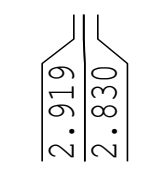


${ }^{1} \mathrm{H}$ NMR (400 MHz, $\mathrm{CDCl}_{3}$ ) of $\mathbf{S 2 3}$ :

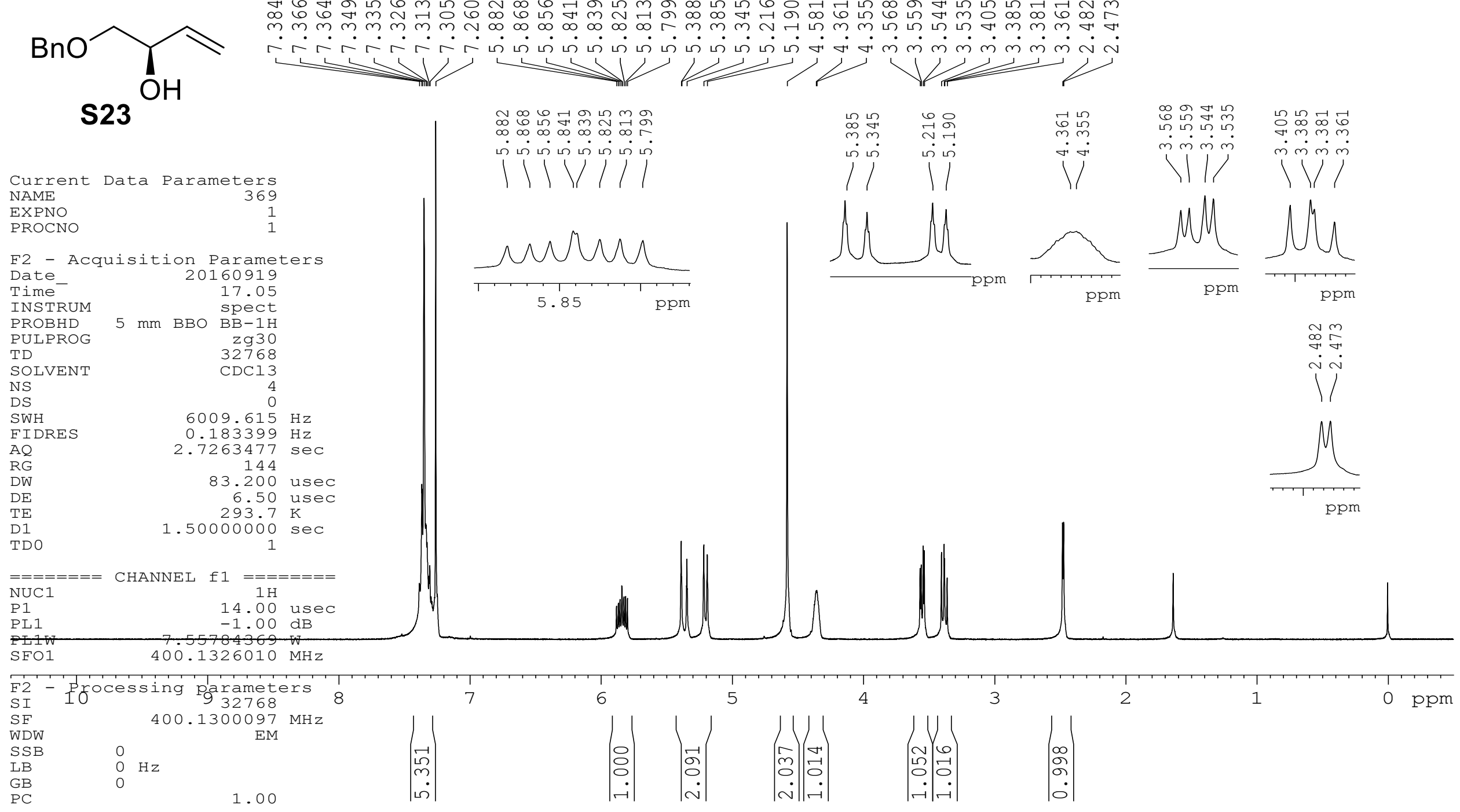


${ }^{1} \mathrm{H}$ NMR $\left(400 \mathrm{MHz}, \mathrm{CDCl}_{3}\right)$ of $\mathbf{1 0}$ :

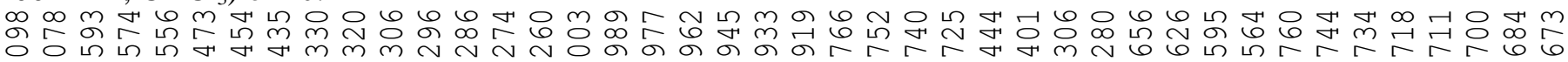

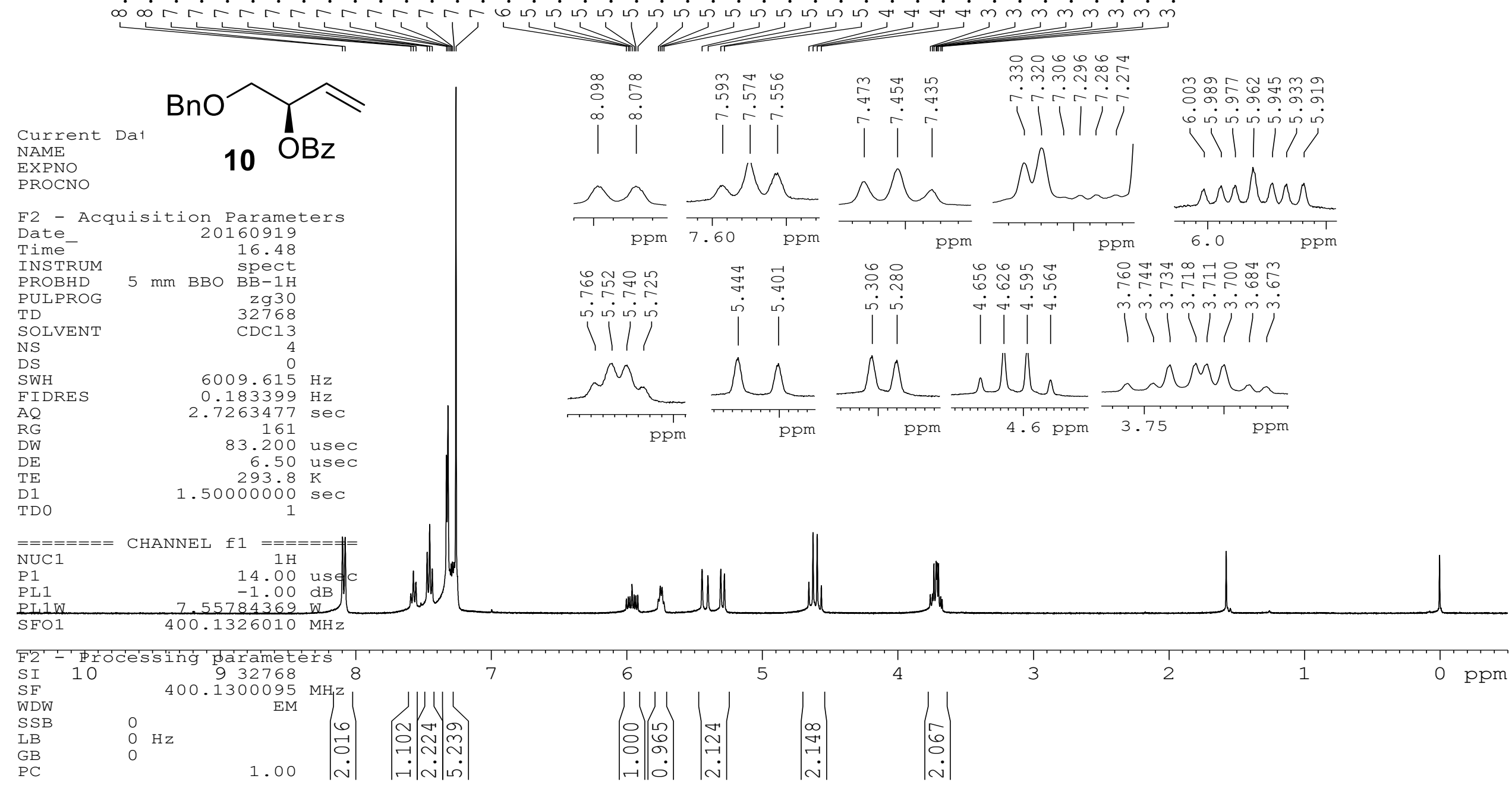


${ }^{13} \mathrm{C}$, DEPT-90 and DEPT-135 NMR (101 MHz, $\left.\mathrm{CDCl}_{3}\right)$ of 10:

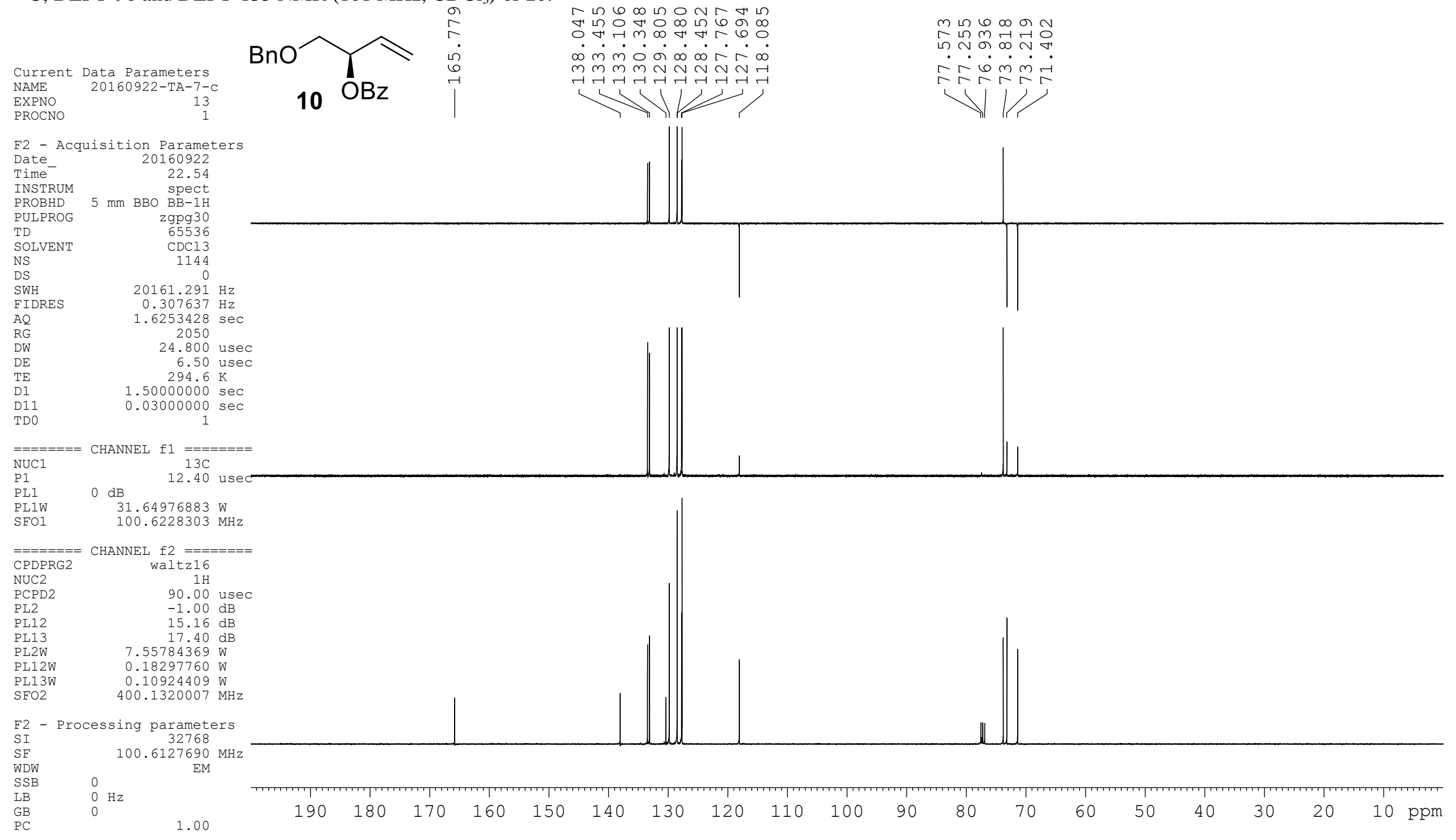




\section{${ }^{1} \mathrm{H}$ NMR $\left(400 \mathrm{MHz}, \mathrm{CDCl}_{3}\right)$ of $\mathbf{1 1}$ :}

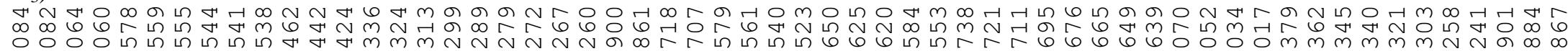
$\circ \circ 0.0 n$.

Current Data Parameters NAME $\quad 91-$ Ta-8-Grubbs EXPNO

F2 - Acquisition Parameters

$\begin{array}{lr}\text { Date } & 20161030 \\ \text { Time } & 18.20\end{array}$

INSTRUM

PROBHD $5 \mathrm{~mm}$ BBO $\begin{array}{r}\text { spect } \\ \mathrm{BB}-1 \mathrm{H}\end{array}$

PULPROG

SOLVENT

NS

SWH

FIDRES

FIDR
AQ
RG
DW

$\mathrm{DE}$

DE
TDO

$=======$ CHANNEL f $1========$

NUC1

$\begin{array}{ll}\text { P1 } & 14.00 \text { usec } \\ \text { PL1 } & -1.00 \mathrm{~dB}\end{array}$

PL1W $7.55784369 \mathrm{~W}$

F2 - Processing parameters

$\begin{array}{ll}\text { SI } & 32768 \\ \text { SF } & 400.1300097 \mathrm{MHz}\end{array}$

WDW

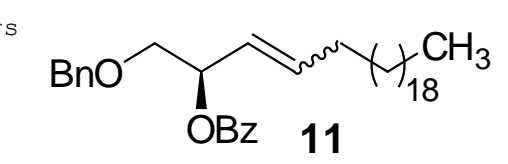

மூ

$\dot{\omega} \dot{6} \dot{0} \dot{0} \dot{0}$

ம மா ம் ம் ம் ம் ம் ம்

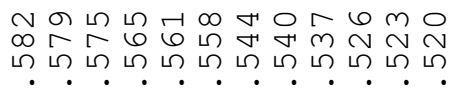

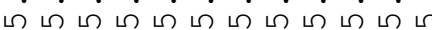
11111

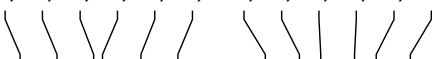

$\backslash 1 / 1 / 1 / 1$

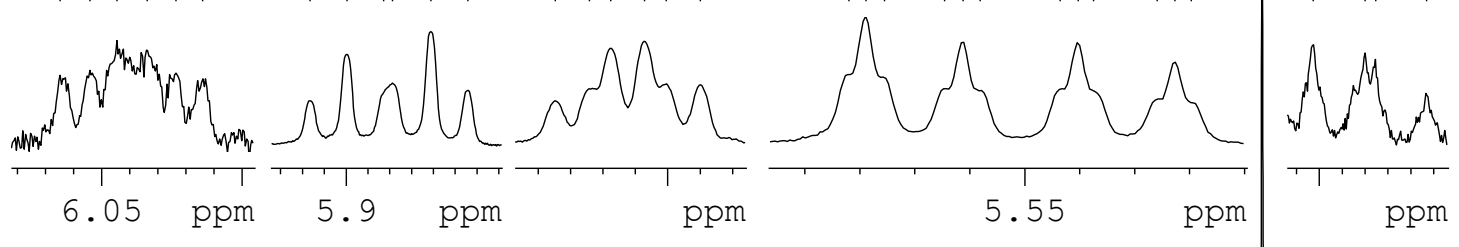

60 กㅁ

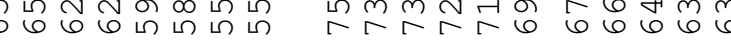

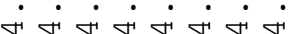

$\dot{m} \dot{m} \dot{m} \dot{m} \dot{m} \dot{m} \dot{m} \dot{m} \dot{m}$

on

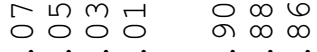

$\sim \sim \sim \sim 00$

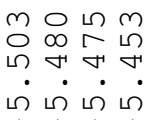

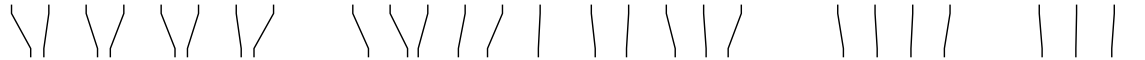

$\begin{array}{lll}\mathrm{LB} & \mathrm{O} \mathrm{Hz}\end{array}$

1.00
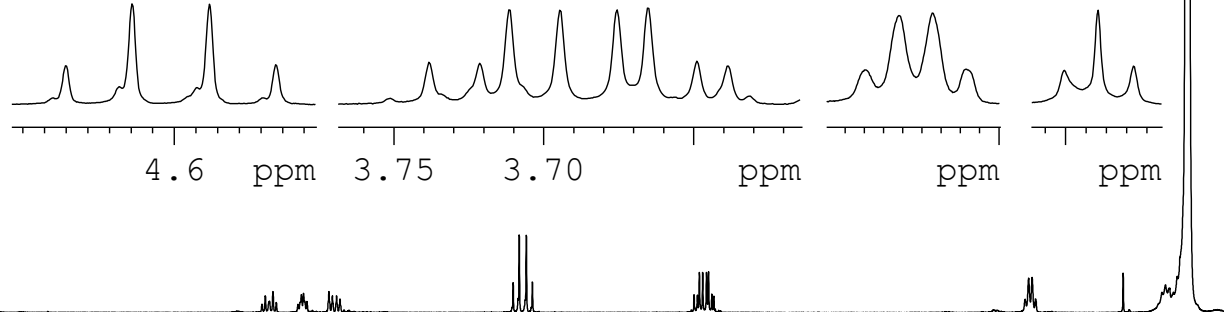

$\begin{array}{lll}10 & 0 \\ 10 & 9\end{array}$

7

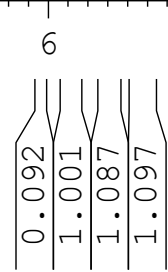

5

4

3

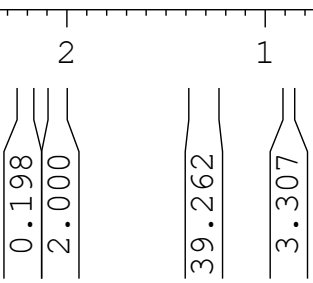


${ }^{13} \mathrm{C}$, DEPT-90 and DEPT-135 NMR (101 MHz, $\left.\mathrm{CDCl}_{3}\right)$ of 11:

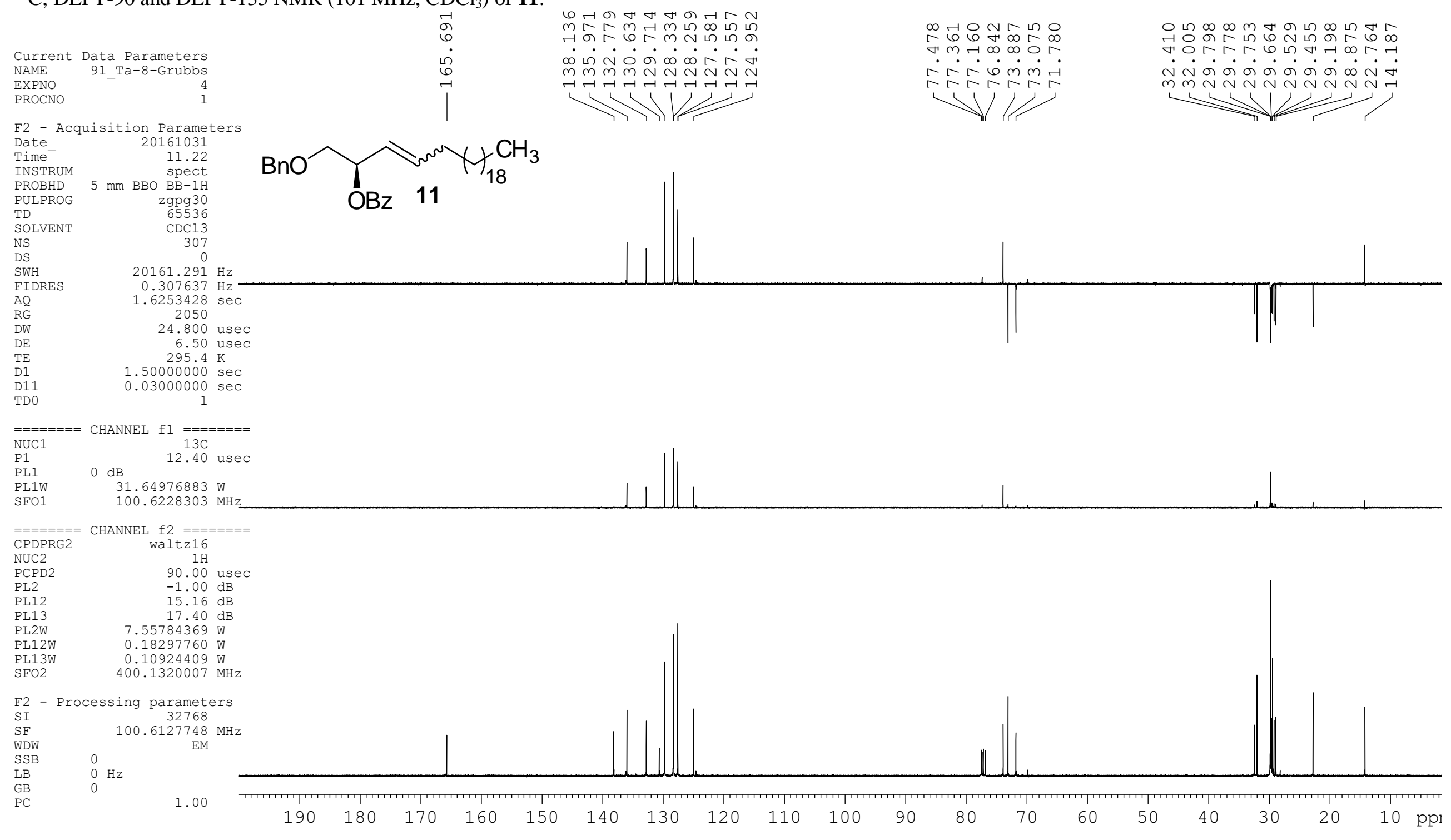




\section{${ }^{1} \mathrm{H}$ NMR (400 MHz, $\mathrm{CDCl}_{3}$ ) of 12:}

$\begin{array}{ll}\text { Current Data Parameters } \\ \text { NAME } & 92 \mathrm{~b} \text {-Ta-9-H2-MeO }\end{array}$

2b_Ta-9-H2-MeO

EXPNO

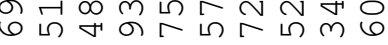
$\infty \infty \infty r a r r r$

31

F2 - Acquisition Parameters Date__ 20171011

INSTRUM 5 - 13.13

INSTRUM $5 \mathrm{~mm}$ BBO spect

PULPROG

TD

NS

DS

FIDRES

$\mathrm{AQ}$

$\mathrm{RG}$

RG
DW
DE
TE
D1

D1

$======$

NUC1

P1

PL1

SFO1

$\mathrm{zg} 30$
32768

$\mathrm{CDCl} 13$

17
0

$6009.615 \mathrm{~Hz}$

$0.183399 \mathrm{~Hz}$ $2.7263477 \mathrm{sec}$

83.200 usec

6.50 usec

$1.50000000 \mathrm{sec}$

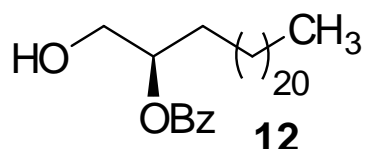

$1 \mathrm{H}$
$14.00 \mathrm{use}$
$-1.00 \mathrm{~dB}$

7.55784369

$400.1326010 \mathrm{MHz}$
ம의 年

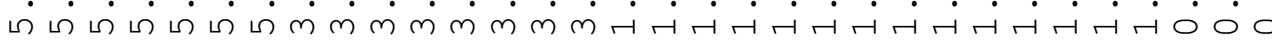

$\longrightarrow+1$

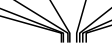

ஜூำ

$\dot{n} \dot{n} \dot{n} \dot{n} \dot{n} \dot{n} \dot{m} \dot{m} \dot{m} \dot{m} \dot{m} \dot{m}$

1111

$\backslash 11111$

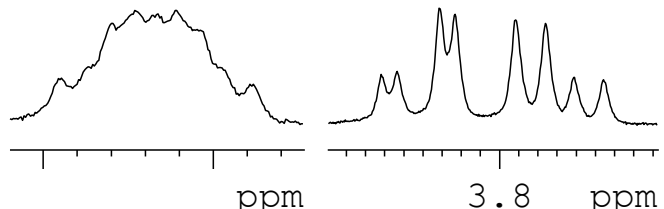

F2 - Processing parameter

32768

$\begin{array}{ll}\mathrm{SF} & 400.1300098 \mathrm{MHz}\end{array}$

LB

GB
PC

0
0
0

(1.00)




\section{${ }^{1} \mathrm{H}$ NMR $\left(400 \mathrm{MHz}, \mathrm{CDCl}_{3}\right.$ ) of $\mathbf{1 6}$ :}

Current Data Parameters NAME 93 Lyx-a hydro-Bz-C24 EXPNO

F2 - Acquisition Parameters Date_ 20180419

Time

INSTRUM

PROBHD

PULPROG

SOLVENT

NS

DS
SWH
FIDRES

$\mathrm{AQ}$

RG

$\mathrm{DW}$

TE

TD 1

$====$
NUC1

PL1

PL1W
SFO1

$40.55784369 \mathrm{MHz}$

SI Processing parameter

SF $\quad 400.1300094 \mathrm{MHz}$

WDW

SS

LB

PC

の

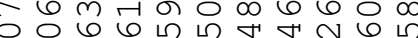

morrararaje

11

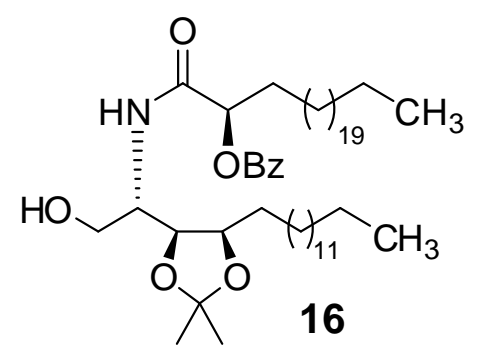

$0.183399 \mathrm{~Hz}$

$7263477 \mathrm{sec}$

83.200 usec

6.50 usec

$.50000000 \mathrm{se}$

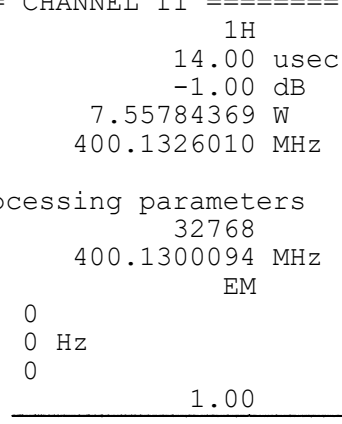

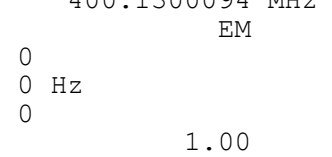

0
0
0

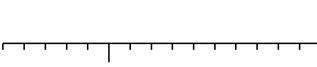

10

9

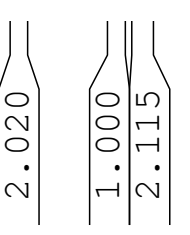

16

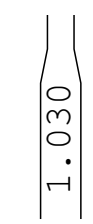

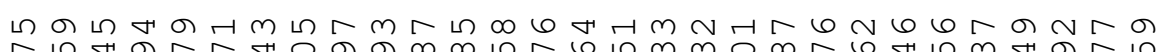
ति

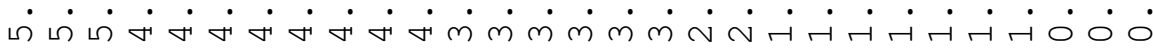

$1+$
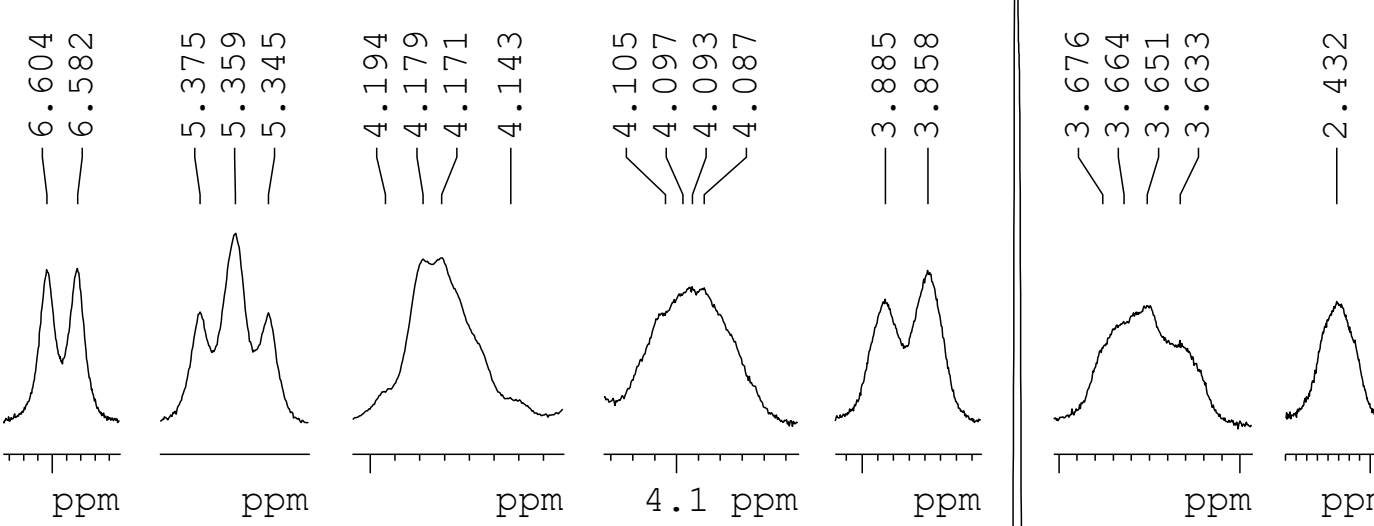

ppm

ppm
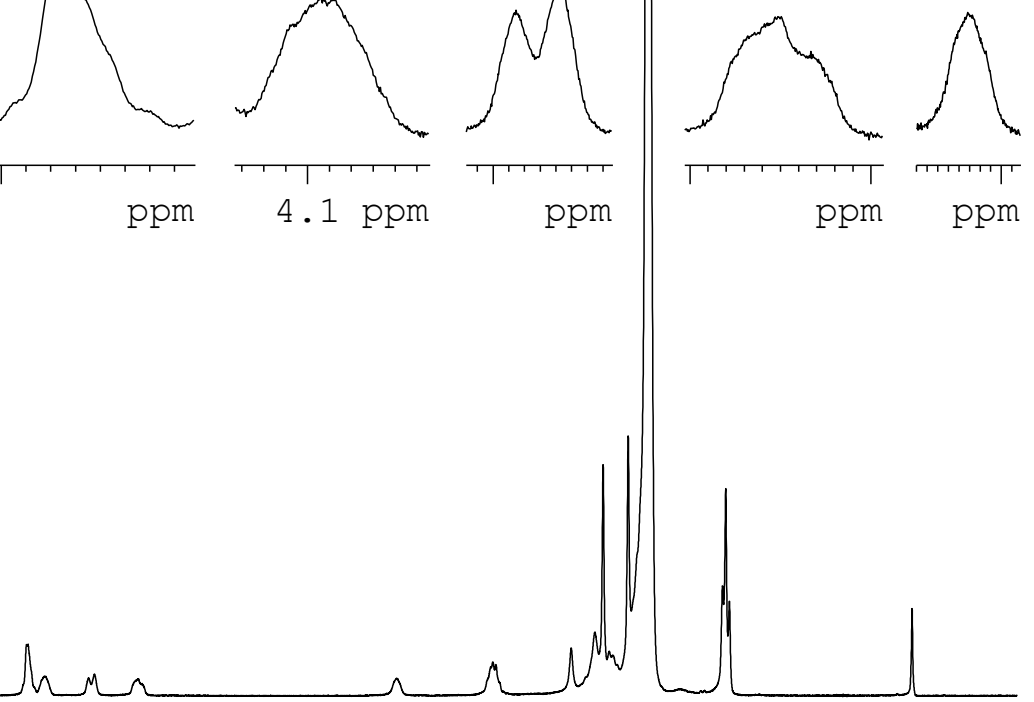

3
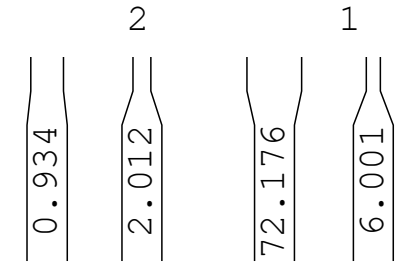

0 ppm

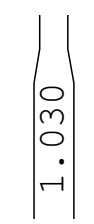

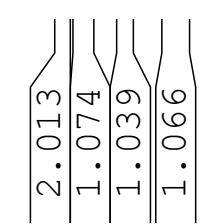

N 
${ }^{13} \mathrm{C}$, DEPT-90 and DEPT-135 NMR (101 MHz, $\left.\mathrm{CDCl}_{3}\right)$ of $\mathbf{1 6}$ :

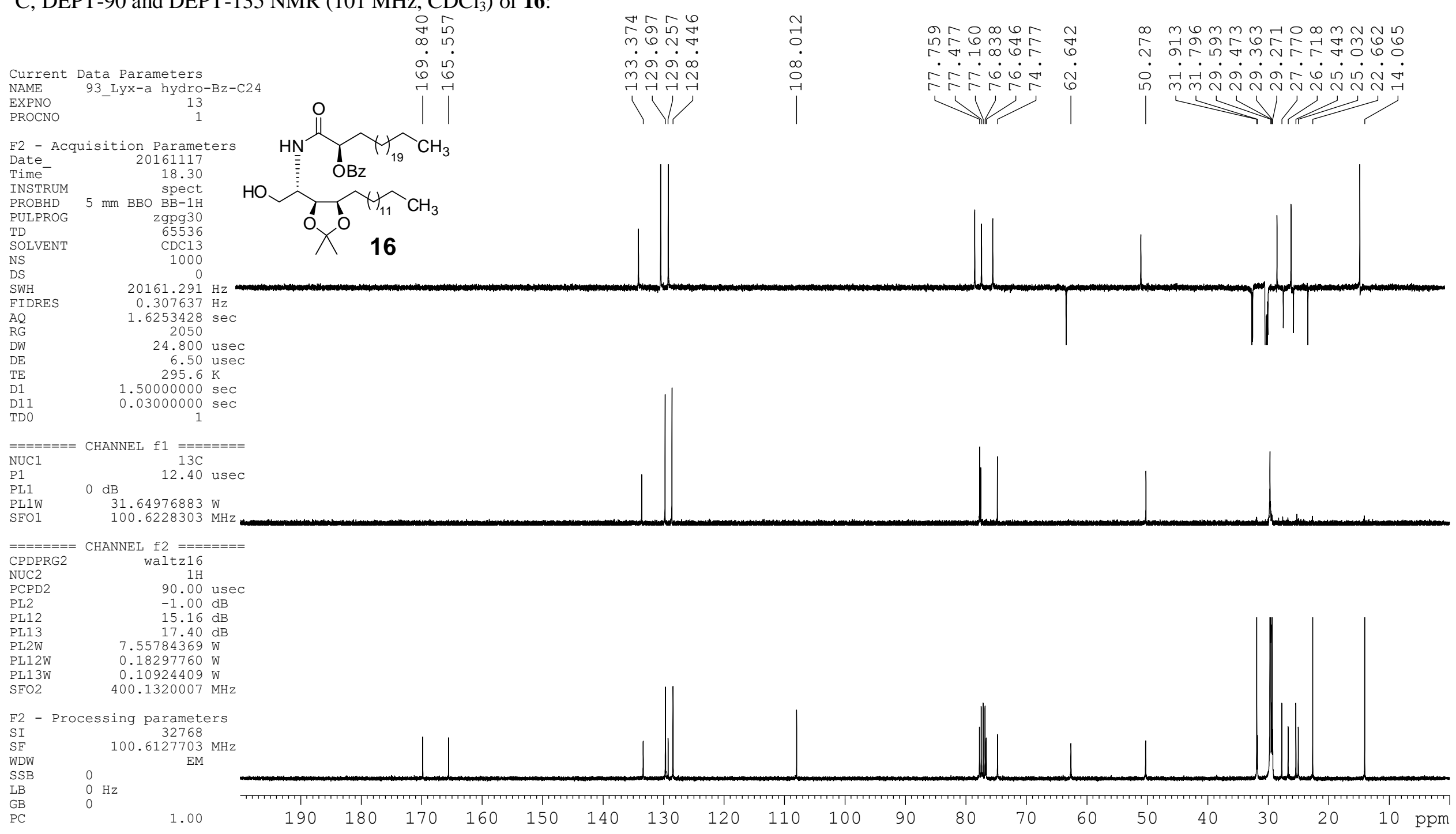




\section{${ }^{1} \mathrm{H}$ NMR $\left(400 \mathrm{MHz}, \mathrm{CDCl}_{3}\right.$ ) of $\mathbf{1 8}$ :}

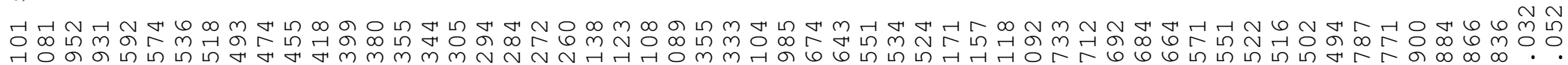

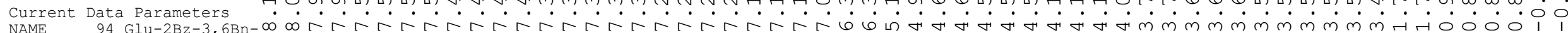

(O) $\begin{array}{lr}\text { EXPNO } & 10 \\ \text { PROCNO } & 1\end{array}$

F2 - Acquisition Parameters

Date_ 20180121

$\begin{array}{ll}\text { Time } & 18.37 \\ \text { INSTRUM } & \text { spect }\end{array}$

PROBHD $5 \mathrm{~mm}$ BBO BB-1H

$\mathrm{zg} 30$
32768
$\mathrm{CDCl}$

SOLVENT

NS
DS

64

SWH

FIDRES

$6009.615 \mathrm{~Hz}$ $2.7263477 \mathrm{~Hz}$

36

83.200 usec

$\begin{array}{lr}\text { DW } & 83.200 \text { use } \\ \text { DE } & 6.50 \text { use } \\ \text { TE } & 294.0 \mathrm{~K} \\ \text { D1 } & 1.50000000 \mathrm{sec}\end{array}$

D1 0

$========$ CHANNEL $\mathrm{f} 1========$

$\begin{array}{lr}\mathrm{NUC1} & 1 \mathrm{H} \\ \mathrm{P} 1 & 14.00 \text { usec }\end{array}$

PL1 $\quad-1.00 \mathrm{~dB}$

$\begin{array}{lr}\text { PL1W } & 7.55784369 \mathrm{~W} \\ \text { SFO1 } & 400.1326010 \mathrm{MHz}\end{array}$

F2 - Processing parameters

F2 - Processing parameters

$\begin{array}{lr}\text { SI } & 32768 \\ \text { SF } & 400.1300098 \mathrm{MHz}\end{array}$

$\begin{array}{ll}\text { WDW } & \\ \text { SSB } & 0 \\ \text { LB } & 0\end{array}$

$\begin{array}{lll}\mathrm{LB} & 0 \mathrm{~Hz} \\ \mathrm{~GB} & 0\end{array}$

EM

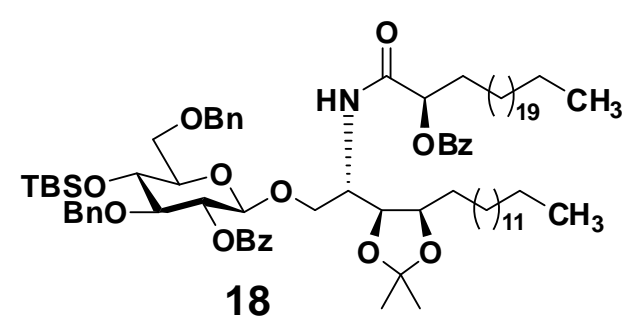

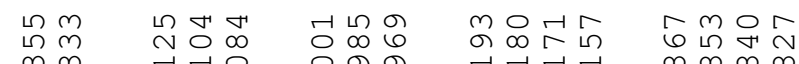

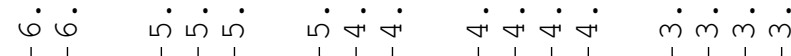

1.00
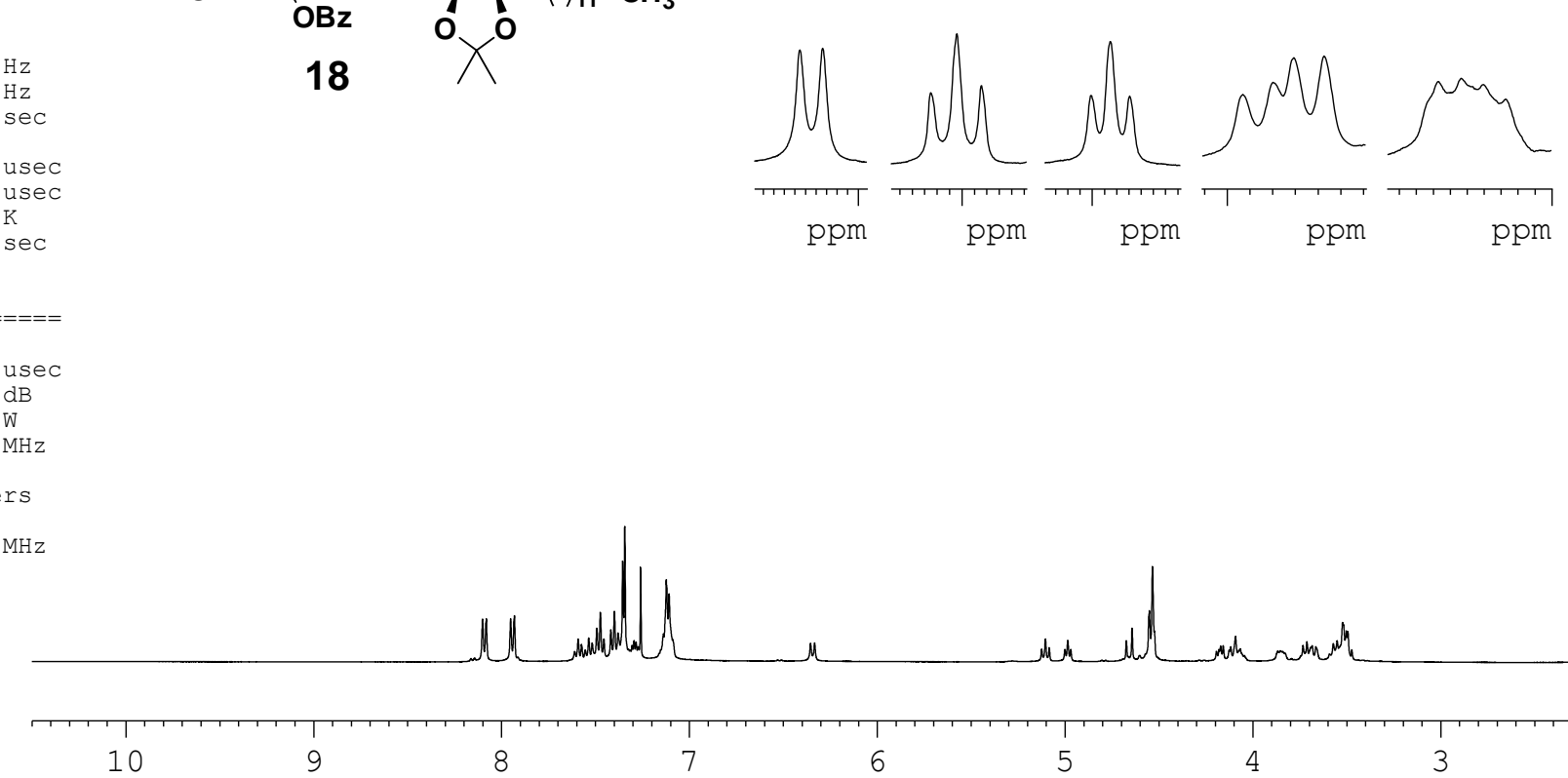

10

18
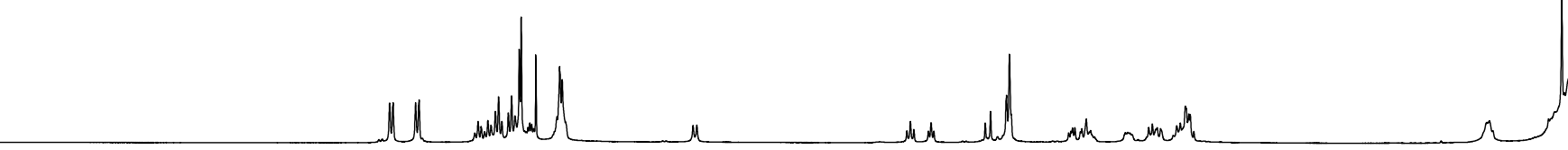

1
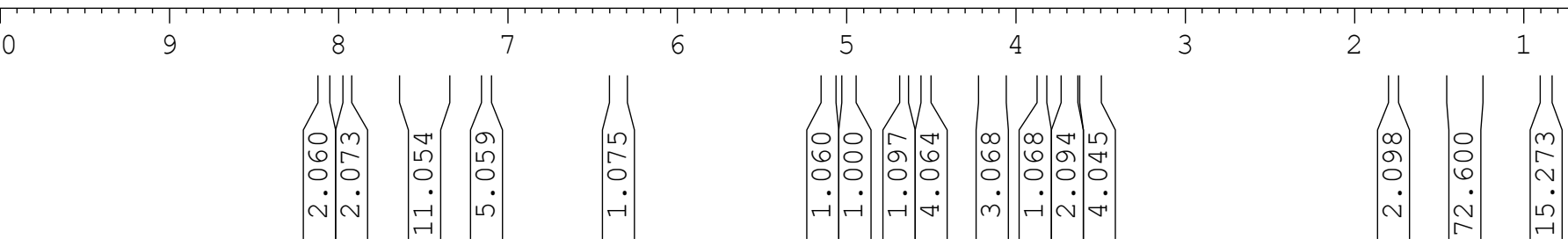
${ }^{13} \mathrm{C}$, DEPT-90 and DEPT-135 NMR (101 MHz, $\mathrm{CDCl}_{3}$ ) of $\mathbf{1 8}$ :

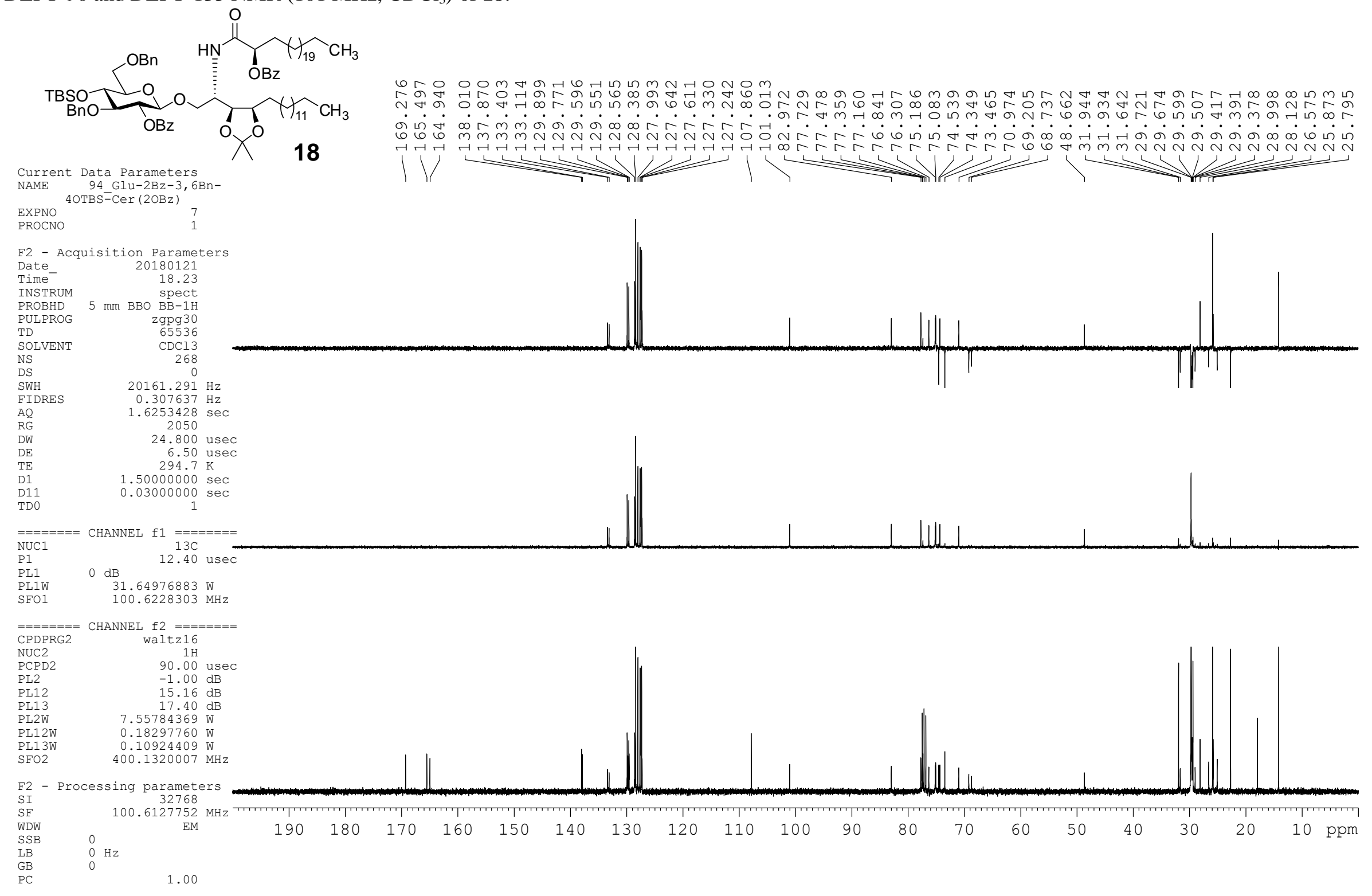




\section{${ }^{1} \mathrm{H}$ NMR $\left(400 \mathrm{MHz}, \mathrm{CDCl}_{3}\right)$ of 19:}

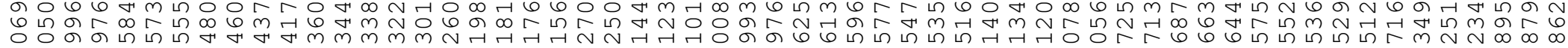
Current Data Parameters NAME $\quad 95 \mathrm{Glu}-2 \mathrm{Bz}-3,6 \mathrm{Bn}-{ }_{4 \mathrm{OH}-\mathrm{Cer}(2 \mathrm{OBz})}$ EXPNO

2-

22 - Acquisition Parameters

$\begin{array}{lr} & 20180126 \\ \text { Time } & 21.14\end{array}$

INSTRUM
PROBHD $5 \mathrm{~mm}$ BBO BB-1H

PROBHD

PULPROG
TD

SOLVEN'

DS

SWH

AQ

$\mathrm{AQ}$
$\mathrm{RG}$
$\mathrm{DW}$

$\mathrm{RG}$
$\mathrm{DW}$
$\mathrm{DE}$

DE

D1
TD0

$\begin{array}{lr}=======\text { CHANNEL } & \text { f1 }======== \\ \text { NUC1 } & 1 \mathrm{H} \\ \text { P1 } & 14.00 \text { usec }\end{array}$

PL1 $1-1.00$ dB

$\begin{array}{lr}\text { PL1W } & 7.55784369 \mathrm{~W} \\ \text { SFO1 } & 400.1326010 \mathrm{MHz}\end{array}$

F2 - Processing parameters

$\begin{array}{lr}\text { SI } & 32768 \\ \text { SF } & 400.1300096 \mathrm{MHz}\end{array}$

$400.1300096 \mathrm{MHz}$ $0.183399 \mathrm{~Hz}$
$2.7263477 \mathrm{sec}$ 144 3.200 use $293.7 \mathrm{~K}$

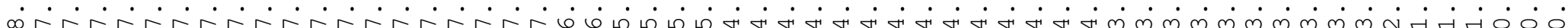

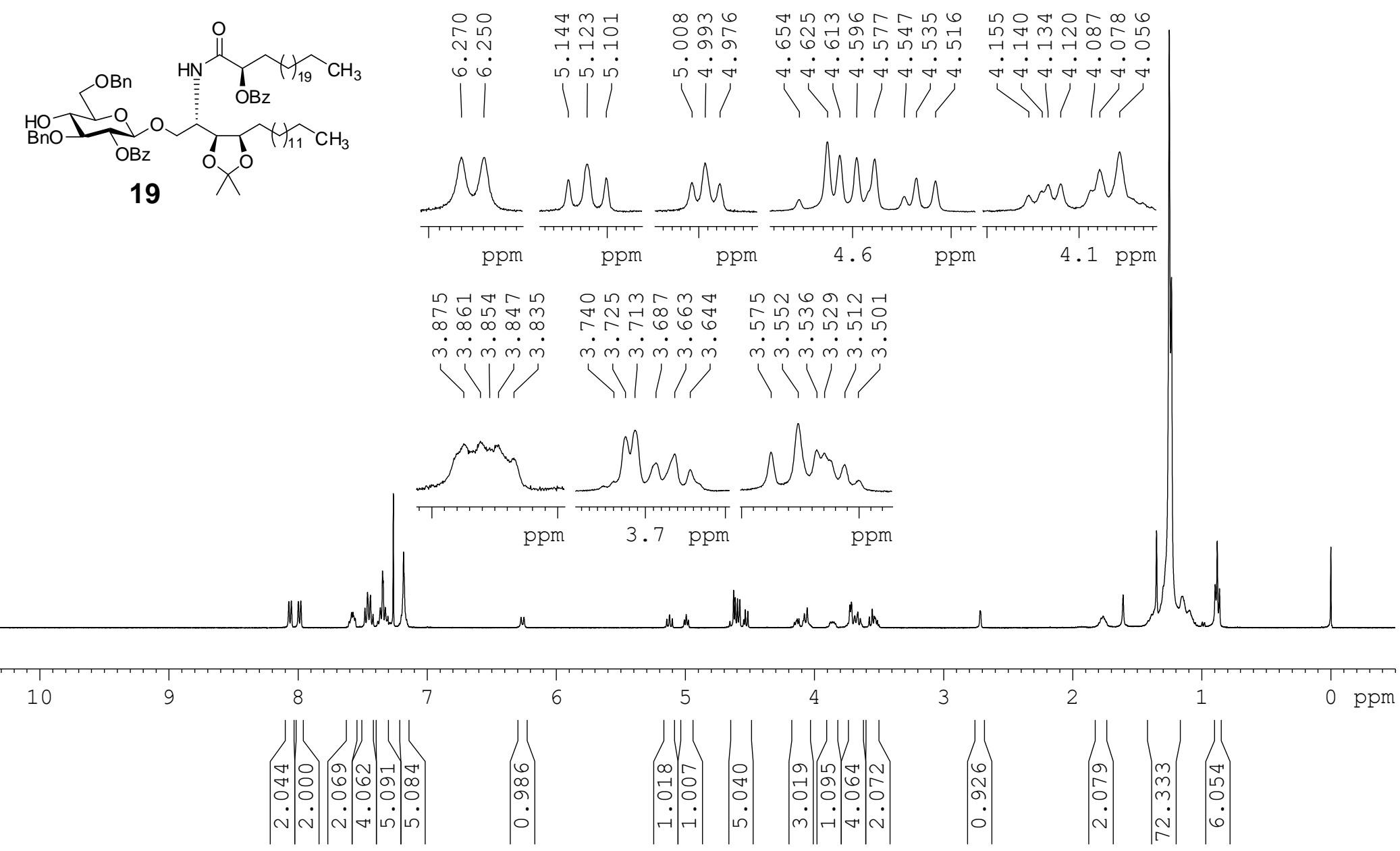


${ }^{13} \mathrm{C}$, DEPT-90 and DEPT-135 NMR (101 MHz, $\mathrm{CDCl}_{3}$ ) of $\mathbf{1 9}$ :

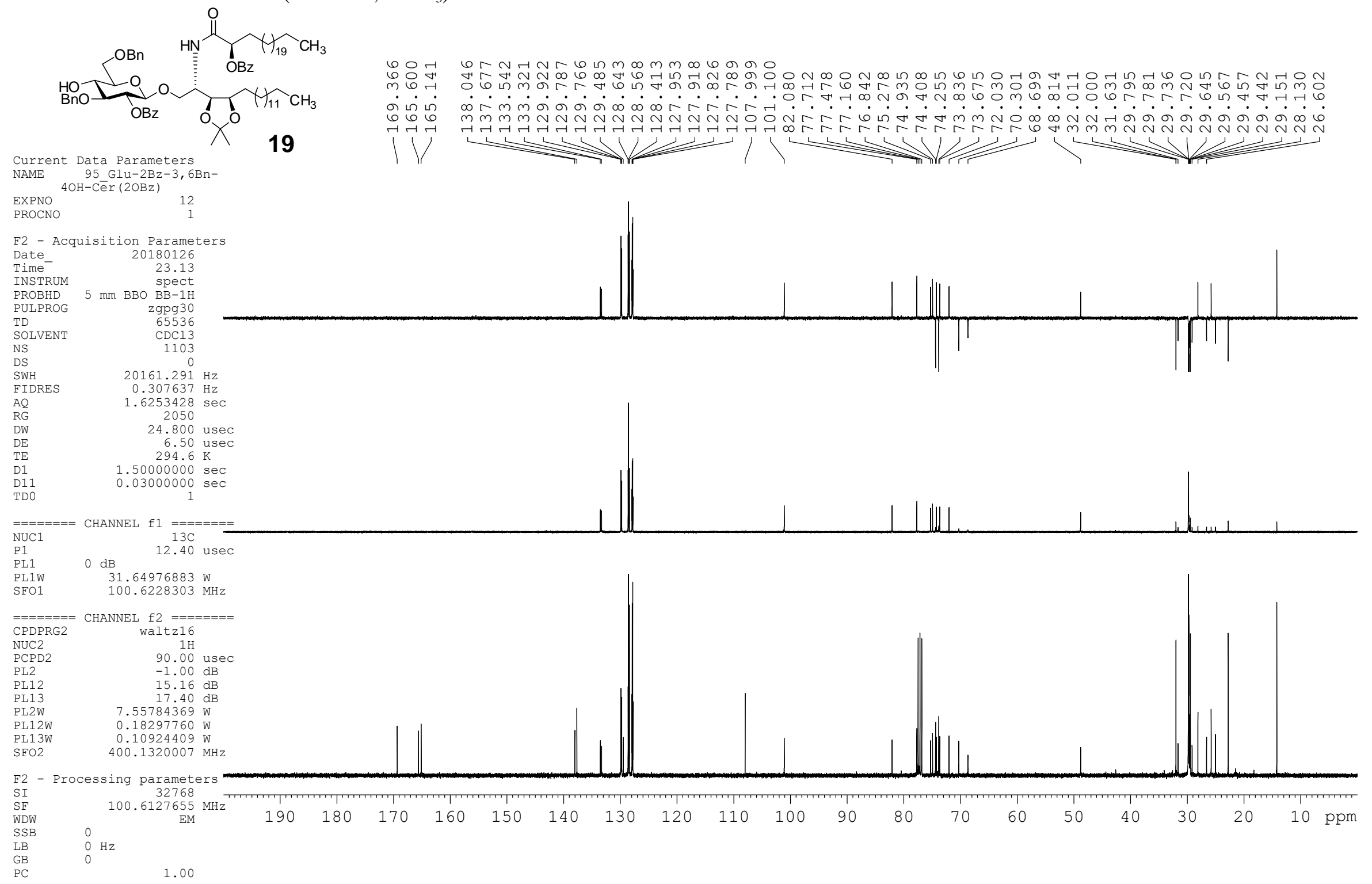




\section{${ }^{1} \mathrm{H}$ NMR $\left(400 \mathrm{MHz}, \mathrm{CDCl}_{3}\right)$ of $\mathbf{4}$}

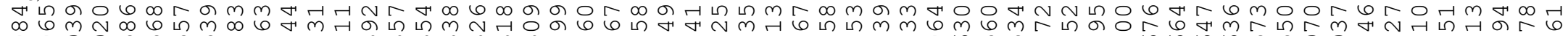
$\left.\begin{array}{lll}0 \\ 0\end{array}\right)$ Current Data Parameters $4 \mathrm{OH}-\mathrm{Cer}(2 \mathrm{OBz})-\mathrm{di} \mathrm{Ac}$ EXPNO
PROCNO

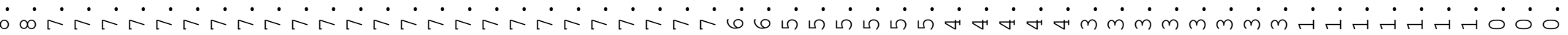

F2 - Acquisition Parameters

Date_ 20180610

$\begin{array}{ll}\text { INSTRUM } & \text { spect } \\ \text { RROBHD } 5 \mathrm{~mm} \text { BBO BB-1H } & \text { z } 3 \text {. }\end{array}$

$\begin{array}{lr}\text { PROBHD } 5 \mathrm{~mm} \text { BBO } & \text { BB-1H } \\ \text { PULPROG } & \mathrm{zg} 30\end{array}$

TD

SOLVEN

DS

$\begin{array}{lr}\text { DS } & 0 \\ \text { SWH } & 6009.615 \mathrm{~Hz} \\ \text { FIDRES } & 0.183399 \mathrm{~Hz}\end{array}$

AQ $\quad 2.7263477 \mathrm{sec}$

$\begin{array}{lr}\text { RG } & 71.8 \\ \text { DW } & 83.200 \text { usec }\end{array}$

DE $\quad 6.50$ use

$\begin{array}{lr}\mathrm{TE} & 294.2 \mathrm{~K} \\ \mathrm{D} 1 & 1.5000000 \mathrm{sec}\end{array}$

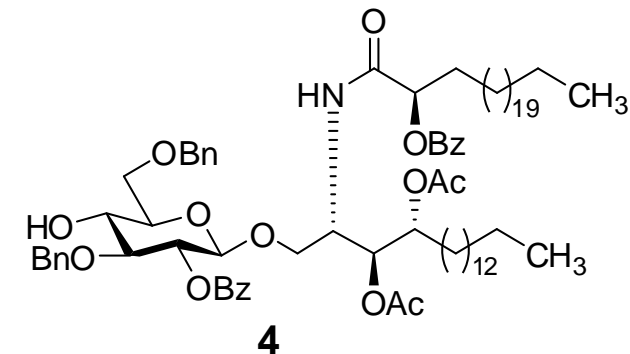

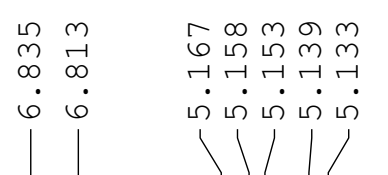

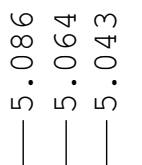

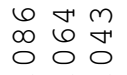

느요요

क ने के ने क.
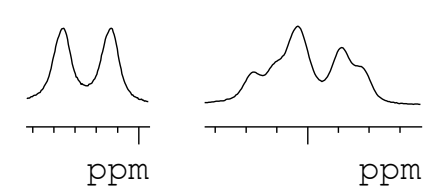

$\frac{\Lambda U}{\mathrm{ppm}} \frac{\Lambda}{\mathrm{ppm}}$

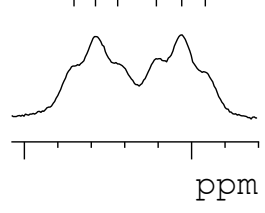

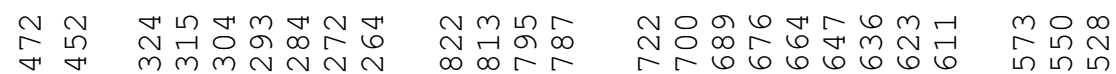

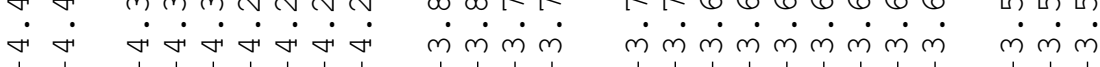

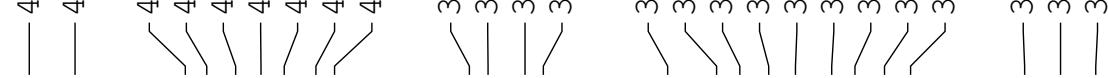

$======$ CHANNEL $\mathrm{fl}========$
NUC1 $1 \mathrm{H}$
P1
14.00 usec

$\begin{array}{lr}\text { P1 } & 14.00 \text { use } \\ \text { PL1 } & -1.00 \mathrm{~dB}\end{array}$

PL1W $\quad 7.55784369 \mathrm{~W}$

F2 - Processing parameters

$\begin{array}{ll}\mathrm{SI} & 32768 \\ \mathrm{SF} & 400.1300097 \mathrm{MHz}\end{array}$

WDW $\quad 400.1300097$ MM

$\begin{array}{ll}\text { SSB } & 0 \\ \text { LB } & 0 \\ \text { Hz }\end{array}$

GB
PC

1.00

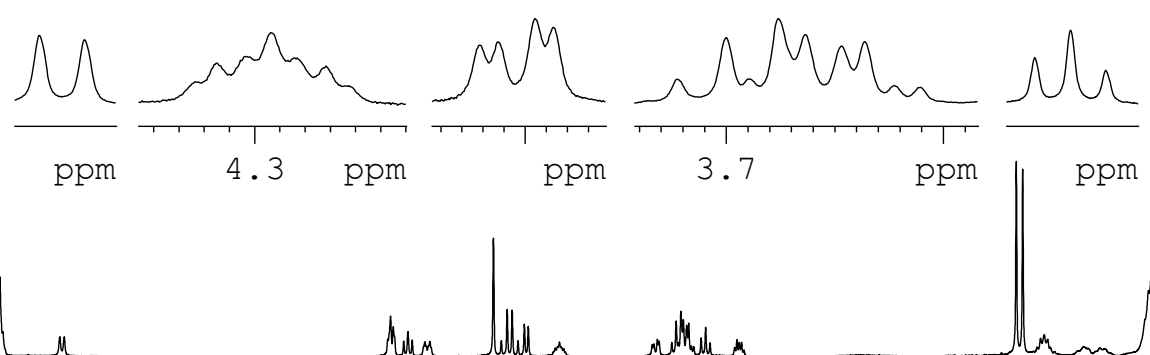

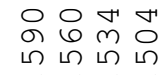

$4 \pi+4$

111

NUu

ppm

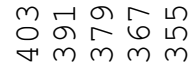

$\dot{m} \dot{m} \dot{m} \dot{m} \dot{m}$

1111

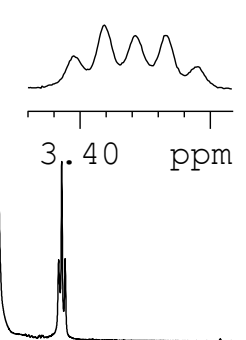

10
10

7

6

4

3

0 ppm
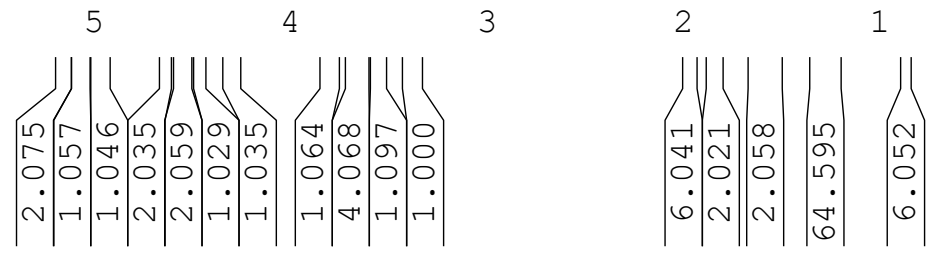
${ }^{13} \mathrm{C}$, DEPT-90 and DEPT-135 NMR (101 MHz, $\left.\mathrm{CDCl}_{3}\right)$ of 4:

Current Data Parameters
NAME Glu-2Bz-3,6Bn-4OH-

Cer (2OBZ) -di AC
EXPNO
PROCNO

F2 - Acquisition Parameters

Date_rition 20180611

$\begin{array}{lr}\text { Time } & 6.05 \\ \text { INSTRUM } & \text { spect } \\ \text { PROBHD } & 5 \mathrm{~mm} \text { BBO BB-1H } \\ \text { BROBH } & \text { Bgpg30 }\end{array}$

PULPROG

TD
SOLVENT

NS

SWH
FIDRES

AQ

RG

$\mathrm{DW}$
$\mathrm{DE}$
$\mathrm{TE}$

TE

D11
TD0

NUC1
P1
PL1 $\quad 0 \mathrm{~dB} \quad 12.40$ usec
P1

$\begin{array}{ll}\text { PL1 } & \mathrm{dB} \\ \text { PL1W } & 31.64976883 \mathrm{~W}\end{array}$

SFO1 $\quad 100.6228303 \mathrm{MHz}$

$=======$ CHANNEL $\mathrm{f} 2=======$

CPDPRG2 waltz16

$\begin{array}{ll}\text { NUC2 } & 90.1 \mathrm{H} \text { usec } \\ \text { PCPD2 } & 90.00 \text { us }\end{array}$

$\begin{array}{ll}90.00 & \text { usec } \\ -1.00 \mathrm{~dB} & \end{array}$

$15.16 \mathrm{~dB}$

$7.55784369 \mathrm{~W}$

$0.18297760 \mathrm{~m}$

$\begin{array}{ll}\text { PL13W } & 0.10924409 \mathrm{~W} \\ \text { SEO2 } & 400.1320007 \mathrm{MHz}\end{array}$

F2 - Processing parameters

$\begin{array}{ll}\text { SI } & 100.6127619 \\ \text { SF } & \mathrm{MHz}\end{array}$

WDW $\quad 100.6127619 \mathrm{MHz}$

$\begin{array}{lll}\text { SSB } & 0 & \\ \text { LB } & 0 & H z \\ \text { GB } & 0\end{array}$

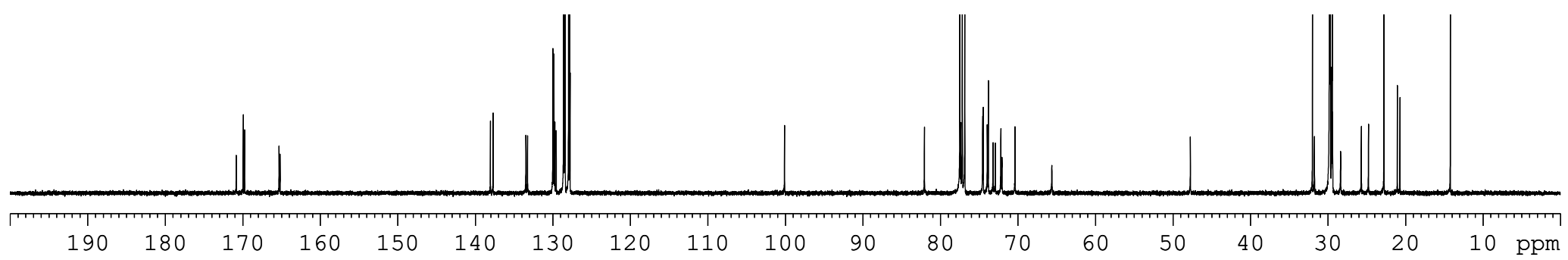




\section{${ }^{1} \mathrm{H}$ NMR (400 MHz, $\mathrm{CDCl}_{3}$ ) of $\mathbf{2 2} \alpha$ :}

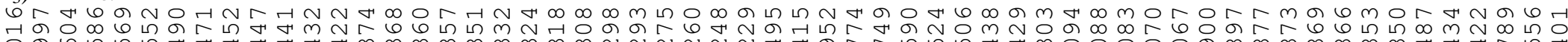
Current Data Parameters

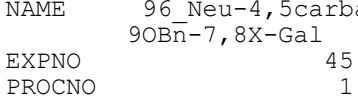

F2 - Acquisition Parameters Date__ 20180126

Time

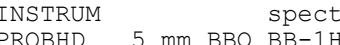
PULPROG

TD

SOLVENT

DS

SWH
FIDRES

$\mathrm{AQ}$

RG

$\mathrm{DW}$
$\mathrm{DE}$
$\mathrm{TE}$

TE
D1
TDO

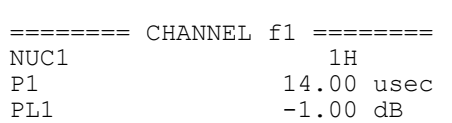

$-1.00 \mathrm{~dB}$
PLI

$\begin{array}{lr}\text { PL1W } & 7.55784369 \mathrm{~W} \\ \text { SFO1 } & 400.1326010 \mathrm{MHz}\end{array}$

F2 - Processing parameter

$\begin{array}{ll}\text { SI } & 32768 \\ \text { SF } & 400.1300095 \mathrm{MHz}\end{array}$

WD

$\begin{array}{ll}\text { SSB } & 0 \\ \text { LB } & 0\end{array}$

$\begin{array}{lll}\mathrm{LB} & 0 \mathrm{~Hz} \\ \mathrm{~GB} & 0\end{array}$

$\mathrm{Hz}$

1.00

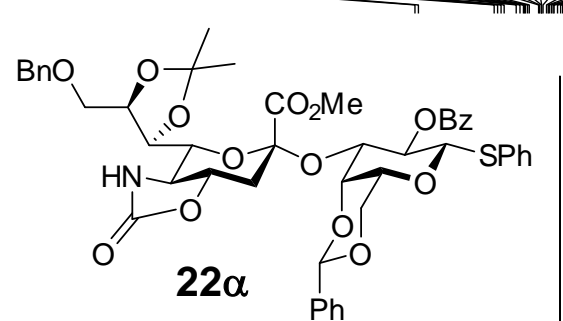

$22 \alpha$

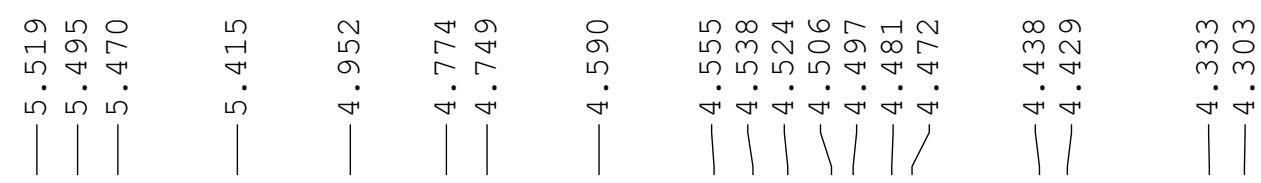

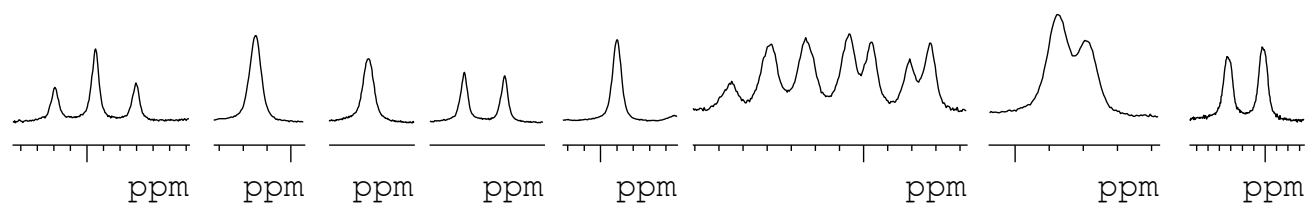

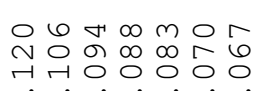

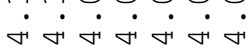

$111 /$

$\begin{array}{lll}-1 & 6 & 0 \\ 0 & 0 & 1 \\ 0 & 0 & 0\end{array}$

$\dot{\nabla} \dot{m} \dot{m}$

$1 / 1$

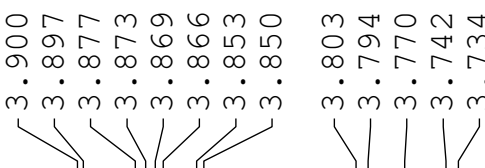

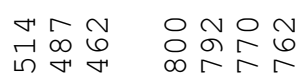

$\dot{m} \dot{m} \dot{\sim} \dot{\sim} \dot{\sim} \dot{\sim}$

$|1| \mid 1$

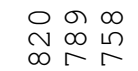

में

i

$\aleph^{N} \underbrace{}_{1,1}$

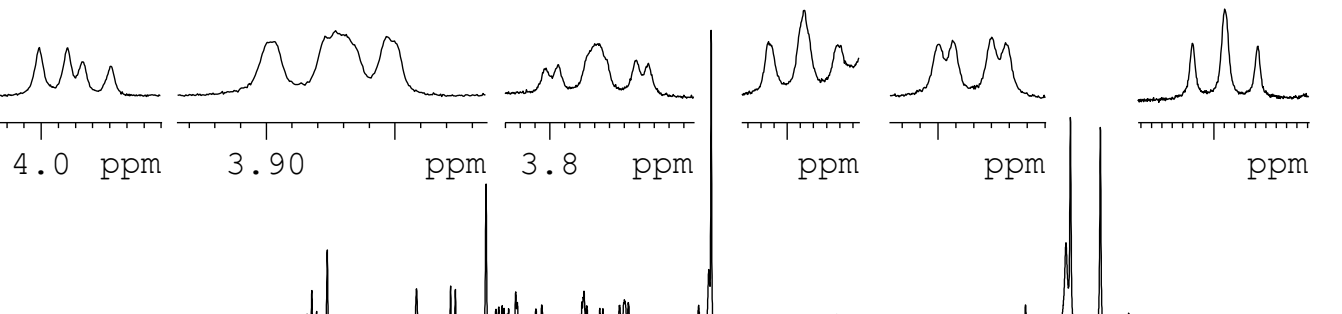

\section{T1 1}

10

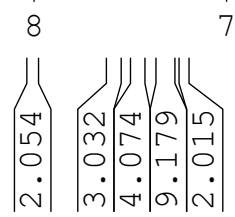

6

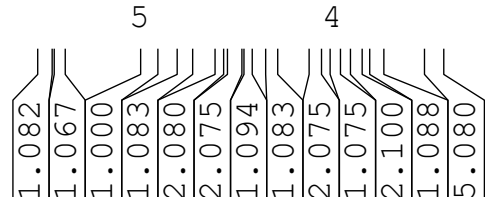

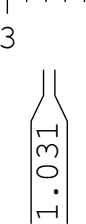


${ }^{13} \mathrm{C}$, DEPT-90 and DEPT-135 NMR (101 MHz, $\left.\mathrm{CDCl}_{3}\right)$ of $\mathbf{2 2} \alpha$ :

$\mathrm{BnO}$

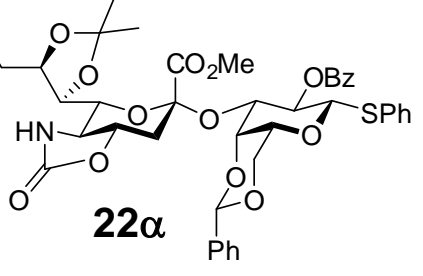

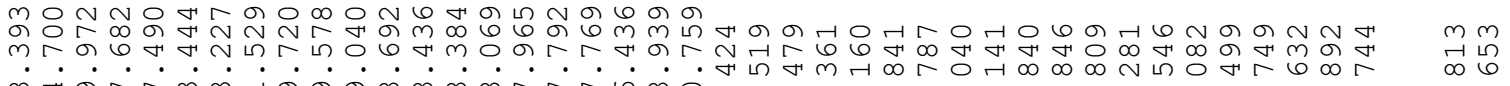

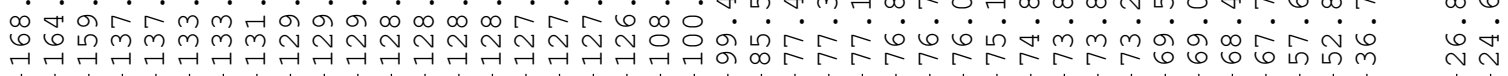

Current Data Parameters
NAME

EXPNO $90 \mathrm{Bn}-7,8 \mathrm{X}-\mathrm{Gal} 52$

F2 - Acquisition Parameters

Date_r 20180127

Time
INSTRUM

INSTRUM
PROBHD

PROBHD
PULPROG

SOLVENT

NS
DS
SWH

SWH
FIDRES
AQ

$\mathrm{AQ}$
$\mathrm{RG}$

$\mathrm{RG}$
$\mathrm{DW}$
$\mathrm{DE}$
$\mathrm{TE}$

D1
D11
TD0

$=======$ CHANNEL

NUC1
P1

PL1 12.40 usec

$\begin{array}{ll}\text { PL1W } & \mathrm{dB} \\ \text { PLI } & 31.64976883 \mathrm{~W} \\ \text { SF1 } & 100.6228303 \mathrm{MHz}\end{array}$

$=======$ CHANNEL $\mathrm{f} 2======$
CPDPRG2

$\begin{array}{lr}\text { CPDPRG2 } & \text { waltz16 } \\ \text { NUC2 } & 1 \mathrm{H} \\ \text { PCPD2 } & 90.00 \text { use } \\ \text { PL2 } & -1.00 \text { us }\end{array}$

$\begin{array}{lr}\text { PCPD2 } & 90.00 \text { usec } \\ \text { PL2 } & -1.00 \mathrm{~dB} \\ \text { PL12 } & 15.16 \mathrm{~dB} \\ \text { PL13 } & 17.40 \mathrm{~dB} \\ \text { PL2W } & 7.55784369 \mathrm{~W} \\ \text { PL12W } & 0.18297760 \mathrm{~W}\end{array}$

$\begin{array}{ll}\text { PL12W } & 0.18297760 \mathrm{~W} \\ \text { PL13W } & 0.10924409 \mathrm{~W}\end{array}$

$\mathrm{SFO} 2$

$0.10924409 \mathrm{~W}$
$400.1320007 \mathrm{MHz}$

F2 - Processing parameters
SI

SI $\quad 32768$

$\begin{array}{ll}\text { SF } & 100.6127829 \\ \text { WDW } & \end{array}$

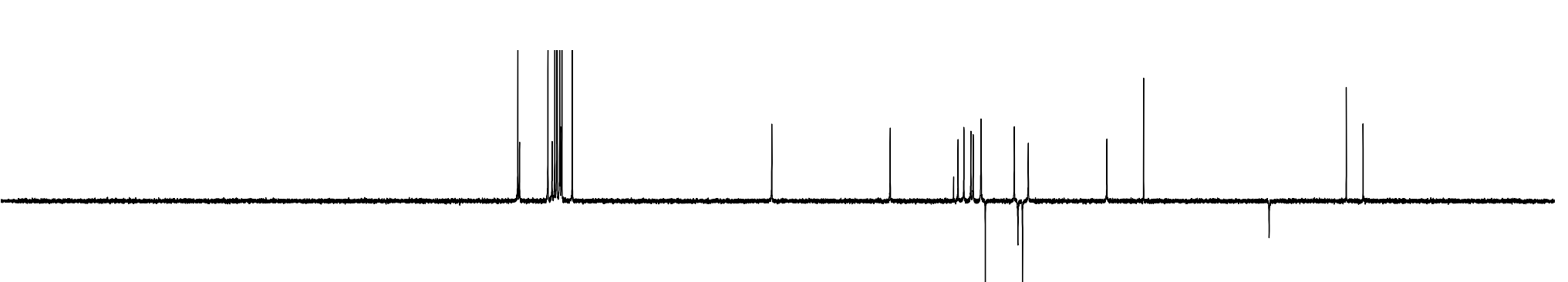




\section{${ }^{1} \mathrm{H}$ NMR $\left(400 \mathrm{MHz}, \mathrm{CDCl}_{3}\right)$ of $\mathbf{2 2 \beta}$}

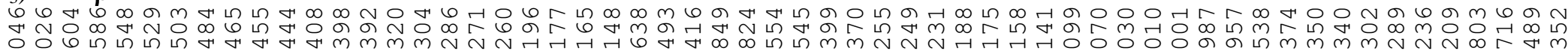

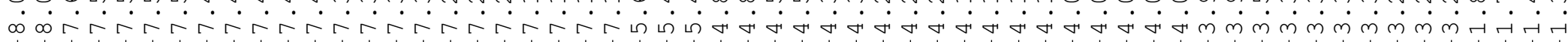
Current Data Parameters EXPNO 7,8X-Gal-b form 6 PROCNO

F2 - Acquisition Parameters $\begin{array}{lr}\text { Date_ruisition } & \text { Parameter } \\ \text { Time } & 23.55\end{array}$ Time-

INSTRUM

PROBHD $5 \mathrm{~mm}$ BBO $\mathrm{BB}-1 \mathrm{H}$

TD

NS
DS

SWH

FIDRES

zg30
32768
CDC13
31

$6009.615 \mathrm{~Hz}$
$0.183399 \mathrm{~Hz}$ $2.7263477 \mathrm{sec}$ 161
83.200 use 83.200 usec
6.50 usec

$\begin{array}{lr}\text { DE } & 6.50 \text { usec } \\ \text { TE } & 293.8 \mathrm{~K} \\ \text { D1 } & 1.5000000 \mathrm{sec}\end{array}$ TDO

$\begin{array}{lr}=======\text { CHANNEL } & \mathrm{f} 1 \mathrm{1}======= \\ \text { NUC1 } & 1 \mathrm{H} \\ \text { P1 } & 14.00 \text { usec }\end{array}$ $\begin{array}{ll}14.00 & \text { usec } \\ \text { PL1 } & -1.00 \mathrm{~dB}\end{array}$ $\begin{array}{lr}\text { PL1W } & 7.55784369 \mathrm{~W} \\ \text { SFO1 } & 400.1326010 \mathrm{MHz}\end{array}$

F2 - Processing parameters $\begin{array}{lr}\text { SI } & 32768 \\ \text { SF } & 400.1300093 \\ \text { MH }\end{array}$

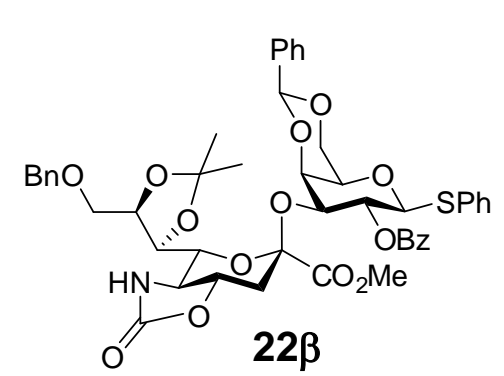

$$
\begin{aligned}
& \text { 아 } 6 \text { ก }
\end{aligned}
$$

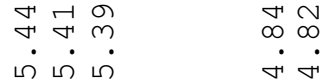

$$
\begin{aligned}
& \text { में }
\end{aligned}
$$

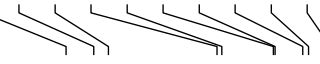

我另

$$
\begin{aligned}
& 6 \\
& 6
\end{aligned}
$$

$m$ वा

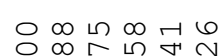
मंब्वं

मं अं अं

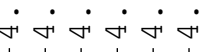

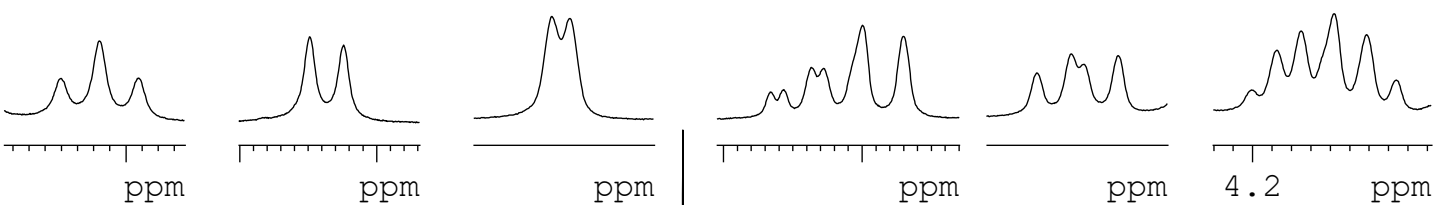
a o o ohr hoO naI

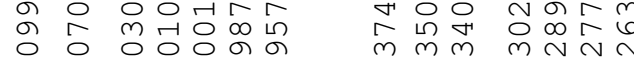

$\dot{\nabla} \dot{\gamma} \dot{\gamma} \dot{\gamma} \dot{r} \dot{m}$

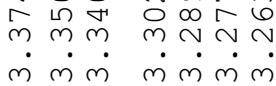
1111 $|1| 1 \mid$
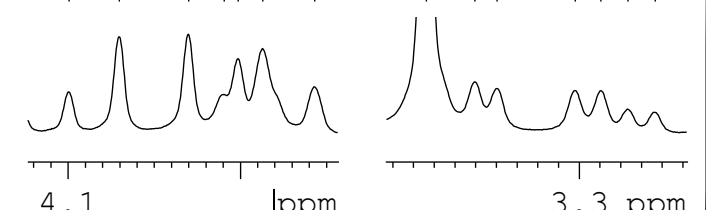

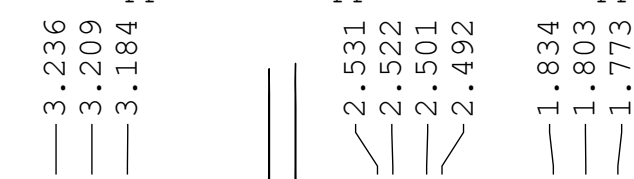
$\mathrm{Hz}$ EM

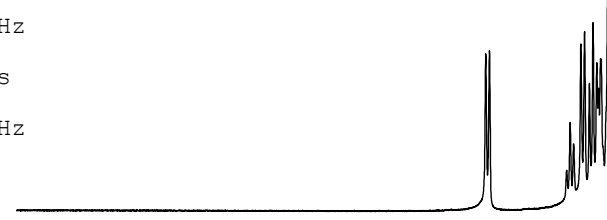

1.00 
${ }^{13} \mathrm{C}$, DEPT-90 and DEPT-135 NMR (101 MHz, $\left.\mathrm{CDCl}_{3}\right)$ of $\mathbf{2 2} \beta$ :

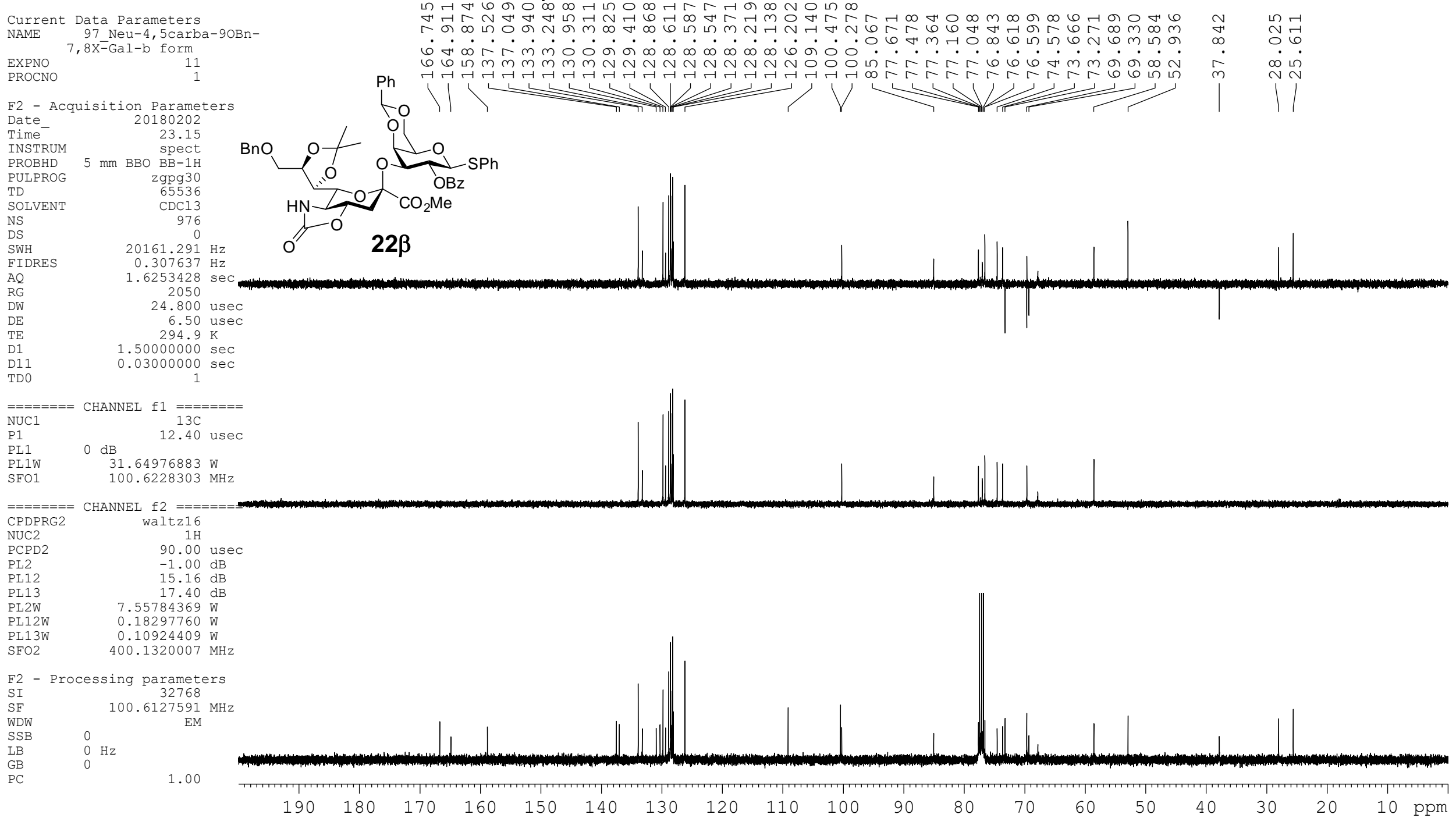


${ }^{1} \mathrm{H}$ NMR $\left(400 \mathrm{MHz}, \mathrm{CDCl}_{3}\right)$ of 23:

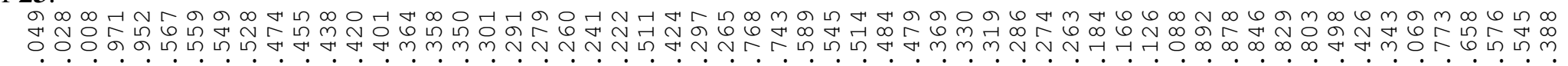

Current Data Parameters

NAME 99_Neu-Neu-Gal-SP

EXPNO
PROCNO

F2 - Acquisition Parameters BzO

Time
Timat
TNSTRUM

INSTRUM

PROBHD
PULPROG

20180204
18.23

$5 \mathrm{~mm}$ BBO $\mathrm{BB}-1 \mathrm{H}$

SD

$\mathrm{BB}-1 \mathrm{H}$
$\mathrm{zg} 30$
32768

SOLVENT
NS

CDC13

SWH

AQ
RG
DW

$\mathrm{DW}$
$\mathrm{DE}$
$\mathrm{TE}$
$\mathrm{D} 1$

TD0

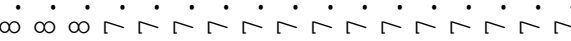

$==\pi==2$ chat

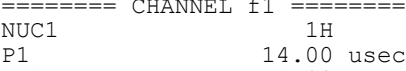

PL1 $\quad-1.00 \mathrm{~dB}$

$\begin{array}{lr}\text { PL1W } & 7.55784369 \mathrm{~W} \\ \text { SFO1 } & 400.1326010 \mathrm{MHz}\end{array}$

F2 - Processing parameters

F2 - Processing parameters

$\begin{array}{lc}\text { SF } & 400.1300094 \mathrm{MHz} \\ \text { WDW } & \text { EM }\end{array}$

$\begin{array}{ll}\text { SSB } & 0 \\ \text { LB } & 0 \\ \text { Hz }\end{array}$

EM

1.00

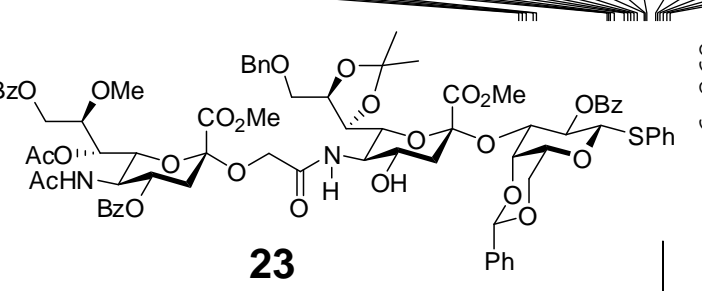

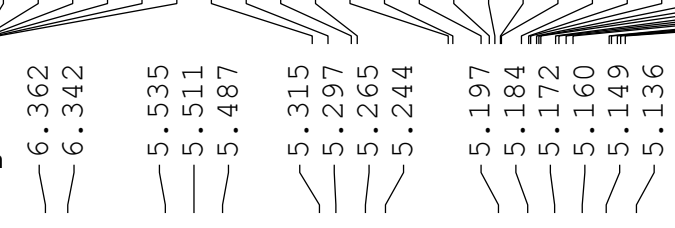

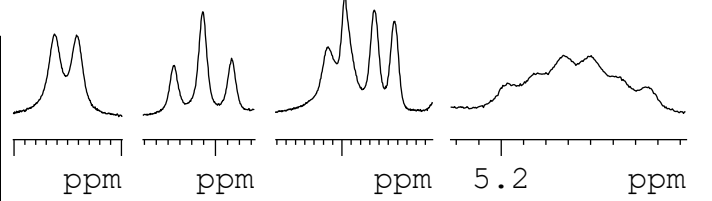

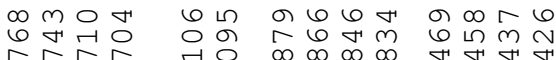

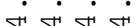

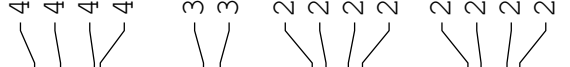

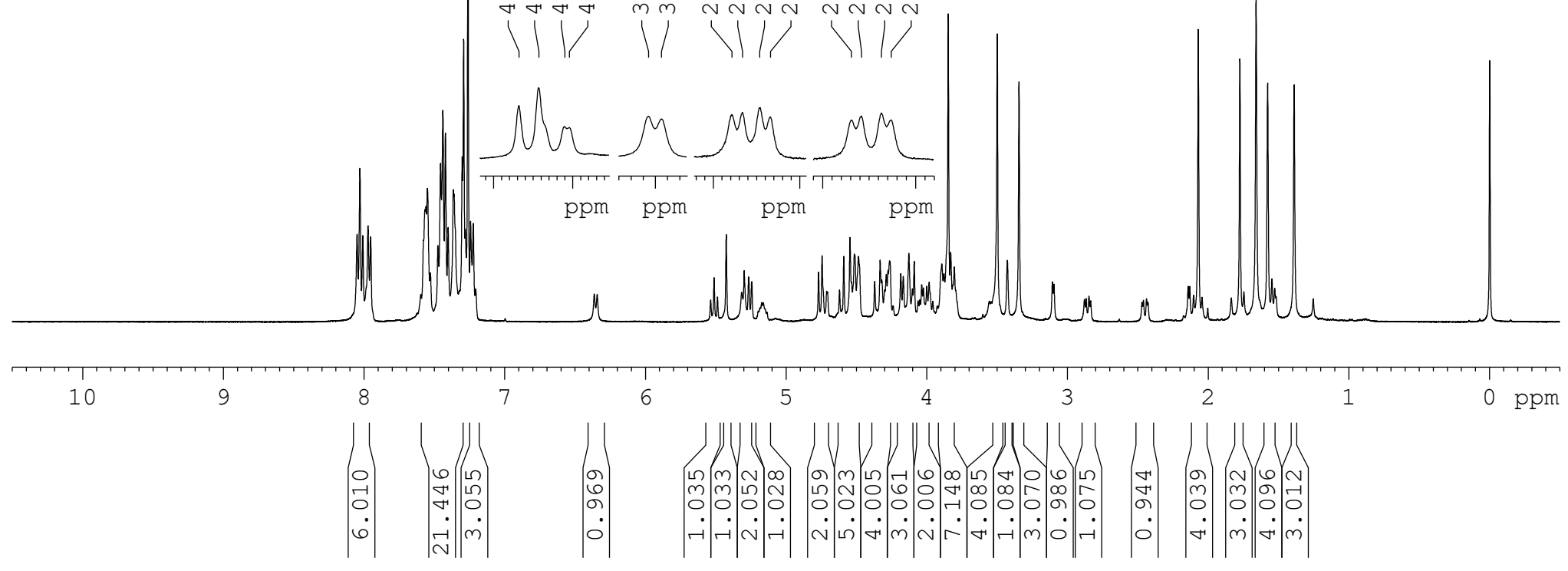


${ }^{13} \mathrm{C}$, DEPT-90 and DEPT-135 NMR (101 MHz, $\left.\mathrm{CDCl}_{3}\right)$ of 23: $\begin{array}{lc}\text { Current } & \text { Data Parameters } \\ \text { NAME } & 99 \text { Neu-Neu-Gal-SPh } \\ \text { EXPNO } & 6 \\ \text { PROCNO } & 1\end{array}$

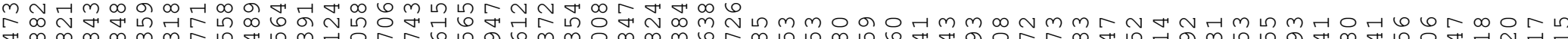

F2 - Acquisition Parameters

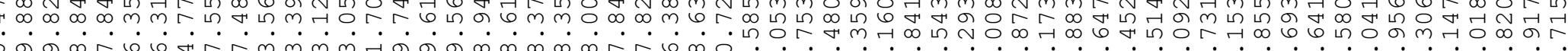

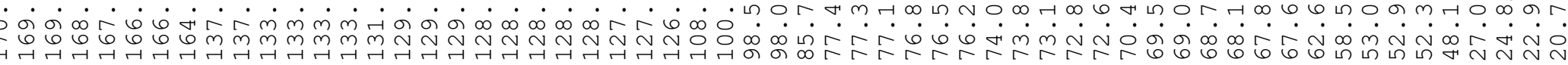

$\begin{array}{lrl}\text { Time } & 0.01 \\ \text { INSTRUM } & \text { BRect }\end{array}$

PROBHD

PULPROG

TD
SOLVENT

NS

DS

FIDRES

$\mathrm{AQ}$

$\mathrm{AQ}$
$\mathrm{RG}$
$\mathrm{DW}$
$\mathrm{DE}$

$\mathrm{DE}$

D1
D11

TDO

$=======$ CHANNEL $\mathrm{f} 1 \quad========$
NUC1 $13 \mathrm{C}$
P1 r 12.40 usec

PL1 $\quad 0 \mathrm{~dB}$

$31.64976883 \mathrm{~W}$

$\mathrm{SFO1} 100.6228303 \mathrm{MHz}$

$======$ CHANNEL $f 2====$
waltz16

$\begin{array}{ll}\text { NUC2 } & 1 \mathrm{H} \\ \text { PCPD2 } & 90.00 \text { usec }\end{array}$

$\begin{array}{ll}\text { PCPD2 } & 90.00 \text { usec } \\ \text { PL2 } & -1.00 \mathrm{~dB} \\ \text { PL12 } & 15.16 \mathrm{~dB}\end{array}$

$\begin{array}{rr}17.16 \mathrm{~dB} \\ \text { PL13 } & 17.40 \mathrm{~dB}\end{array}$

PL2W

PL12W

PL13W

$7.55784369 \mathrm{~W}$

$0.10924409 \mathrm{~W}$

F2 - Processing parameters

$\begin{array}{ll}\text { SI } & 32768 \\ \text { SF } & 100.6127818 \mathrm{MHz}\end{array}$

EM

LB $\quad 0 \mathrm{~Hz}$

(1)

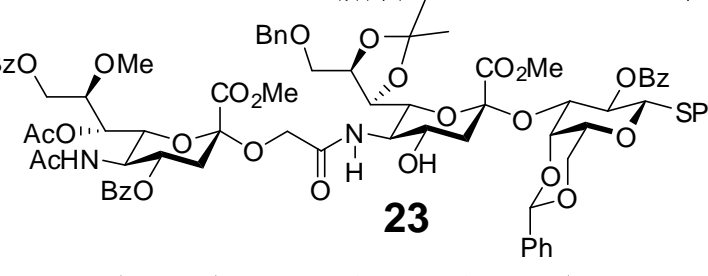

(1)
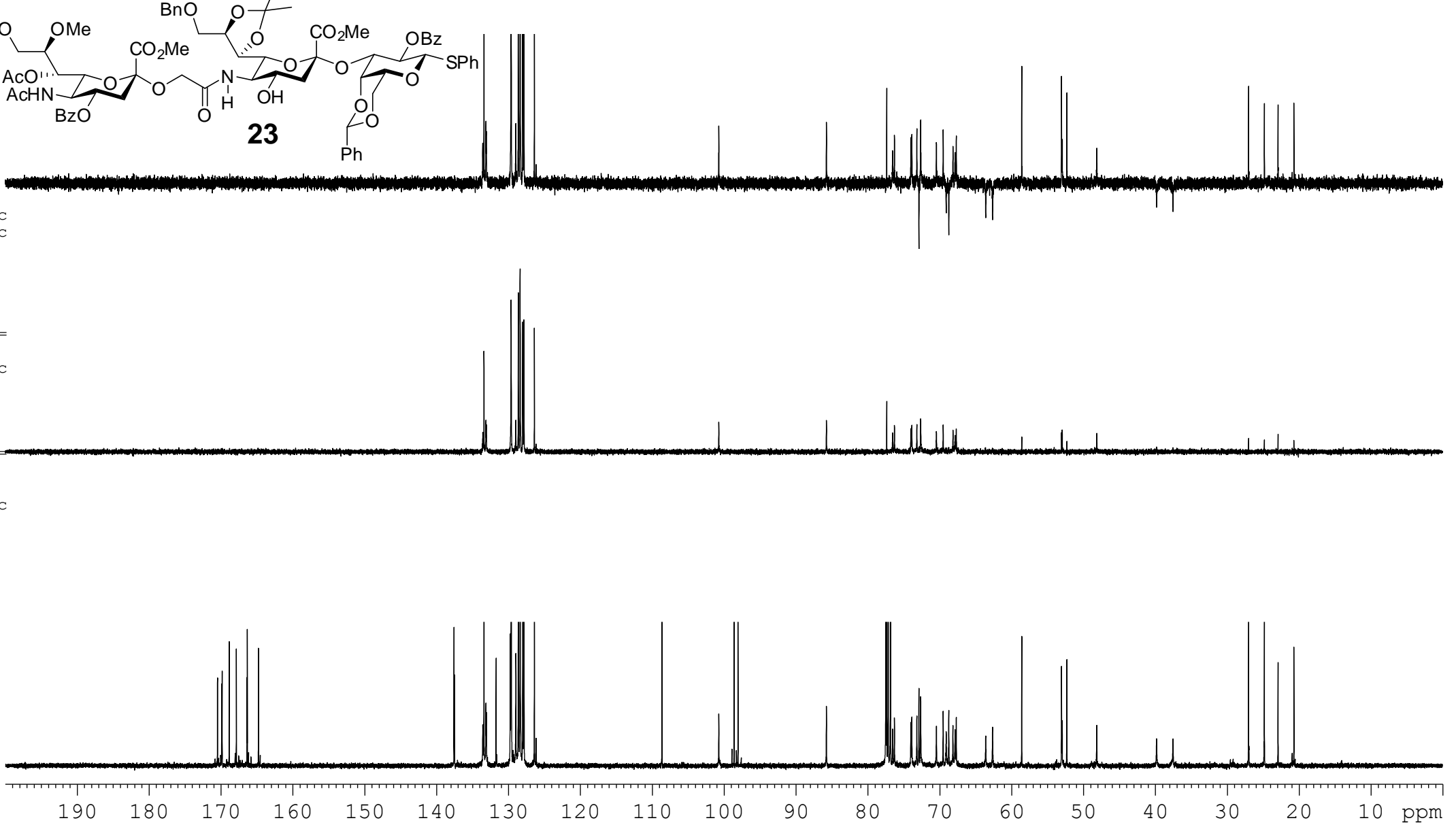
${ }^{1} \mathrm{H}$ NMR $\left(400 \mathrm{MHz}, \mathrm{CDCl}_{3}\right)$ of 24:

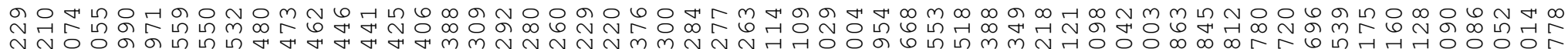

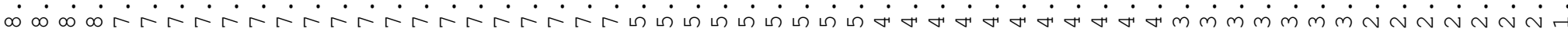

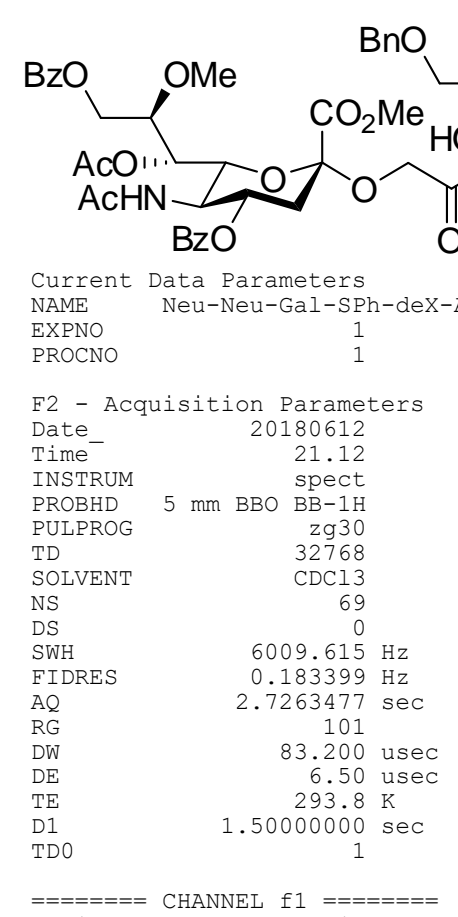
$\begin{array}{ll}=======\text { CHANNEL } & \mathrm{f} 1 \mathrm{l}======= \\ \text { NUC1 } & 1 \mathrm{H} \\ \text { P1 } & 14.00 \text { usec }\end{array}$ $\begin{array}{lr}\text { P1 } 1 & -1.00 \mathrm{~dB} \\ \text { PL1W } & 7.55784369 \mathrm{~W}\end{array}$ $\begin{array}{lr}\text { PL1W } & 7.55784369 \mathrm{~W} \\ \text { SFO1 } & 400.1326010 \mathrm{MHz}\end{array}$ F2 - Processing parameters $\begin{array}{cc}\text { SI } & 32768 \\ \mathrm{SF} & 400.1300093 \mathrm{MHz}\end{array}$ WDW $\quad$ EM $\begin{array}{ll}\text { SSB } & 0 \\ \text { LB } & 0 \\ \text { GB } & 0\end{array}$

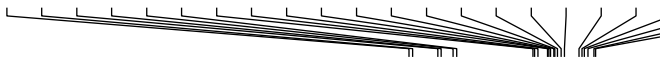

$\mathrm{PC}$

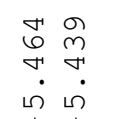

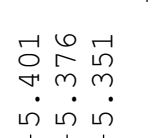
요요

\section{Ha}

4
in i ம் ம் ம் ம் ம் ம் ம்

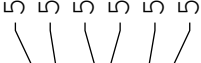

$\prod_{\mathrm{H}}^{\mathrm{N}} \mathrm{OAC}_{\mathrm{A}}$

24
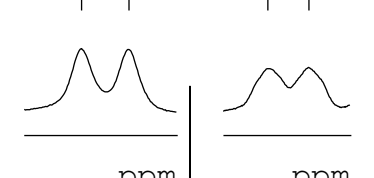

Mh shemang M

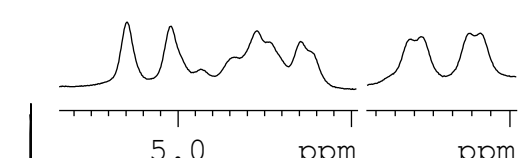

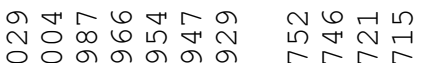
(i)

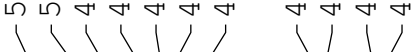
$5.4 \mathrm{ppm} 5.3 \mathrm{ppm} \quad 5.2 \mathrm{ppm} \quad \mathrm{ppm}$
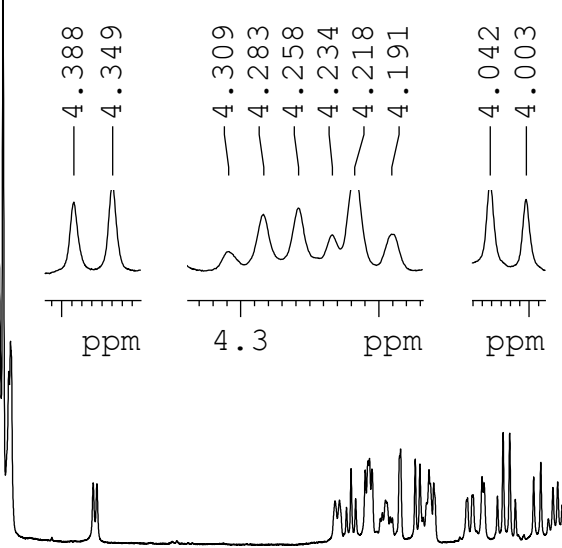

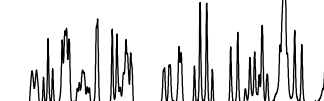
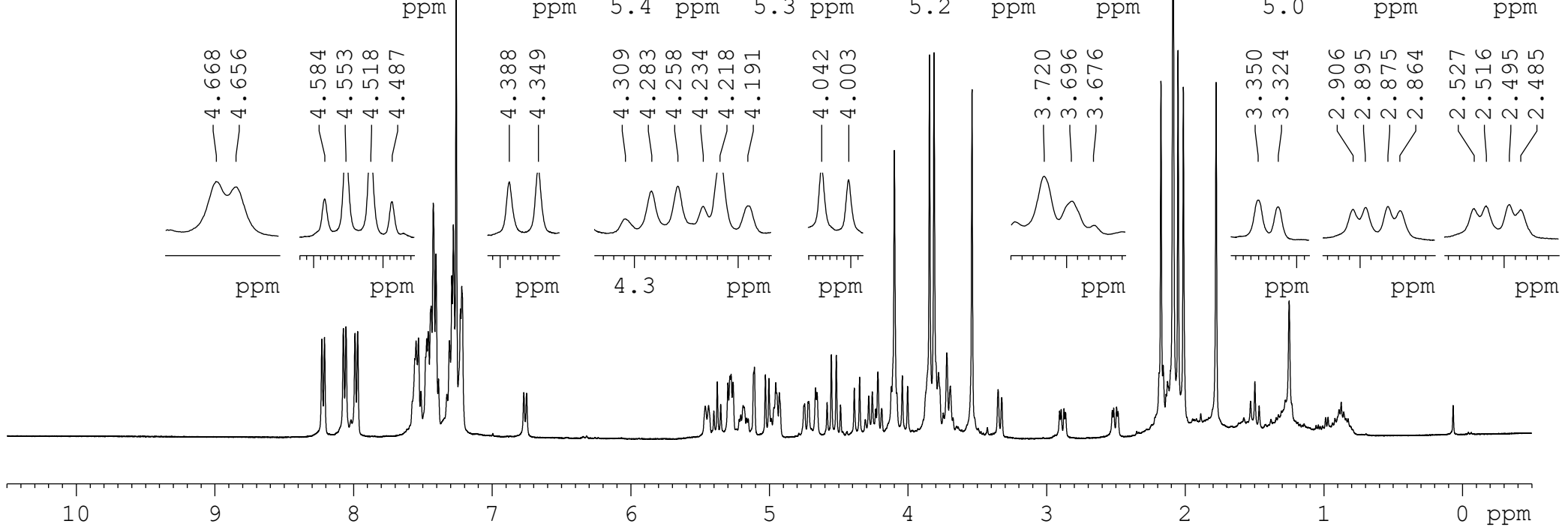

9

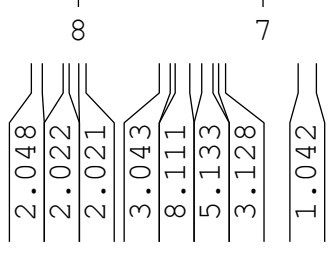

3

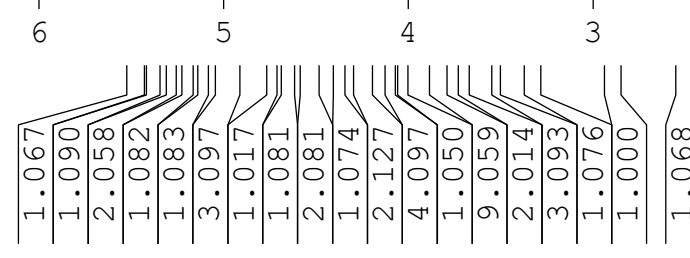


${ }^{13} \mathrm{C}$, DEPT-90 and DEPT-135 NMR (101 MHz, $\left.\mathrm{CDCl}_{3}\right)$ of $\mathbf{2 4}$ :

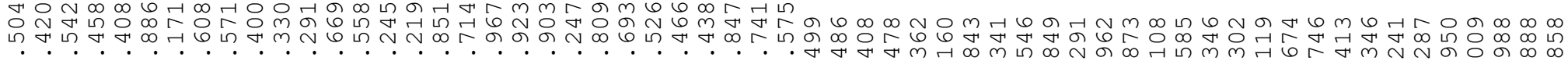

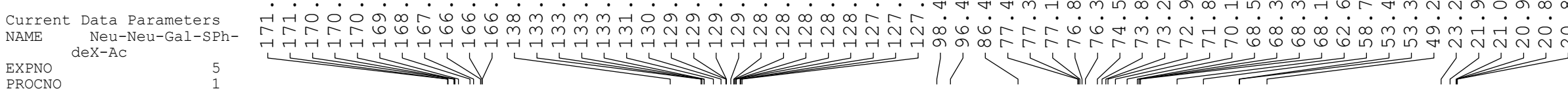

F2 - Acquisition Parameters

$\begin{array}{lr}\text { Date } & 20180613 \\ \text { Time }^{-} & 18.06\end{array}$

spect
INSTRUM
RROBHD $5 \mathrm{~mm}$ BBO BB-1H

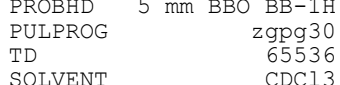

SOLVENT
NS

$\mathrm{CDC13}$
11723

SWH $\quad 20161.291 \mathrm{~Hz}$

$\begin{array}{ll}\text { FIDRES } & 0.307637 \mathrm{~Hz} \\ \mathrm{AD} & 1.6253428 \mathrm{sec}\end{array}$

$\begin{array}{lr}\text { AQ } & 1.6253428 \text { sec } \\ \text { RG } & 2050 \\ \text { DW } & 24.800 \text { usec } \\ \text { DE } & 294.3 \mathrm{~K} \text { Ke }\end{array}$

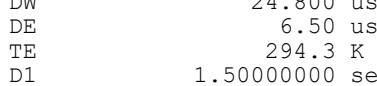

$\begin{array}{ll}\text { D1 } & 1.50000000 \mathrm{se} \\ \text { D11 } & 0.03000000 \\ \text { TDO } & \end{array}$

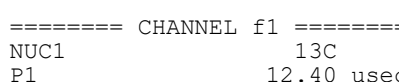

PL1 $0 \mathrm{~dB} \quad 12.40 \mathrm{usec}$

$\begin{array}{ll}\text { PL1W } & 31.64976883 \mathrm{~W} \\ \text { SFO1 } & 100.6228303 \mathrm{MHZ}\end{array}$

$=======$ CHANNEL $f 2=======$
CPDPRG

CPDPRG2
NUC2 2 waltz16
PRD

$\begin{array}{ll}\text { PCPD2 } & 90.00 \text { us } \\ \text { PL2 } & -1.00 \mathrm{~dB} \\ \text { PL12 } & 15.166 \mathrm{~dB} \\ \text { PL13 } & 7.5754 .09 \mathrm{~dB}\end{array}$

PL12
PL13

$\begin{array}{ll}\text { PL2W } & 7.55784369 \mathrm{~W} \\ \mathrm{PL} & \mathrm{T}\end{array}$

$\begin{array}{lr}\text { PL12W } & 0.18297760 \mathrm{~W} \\ \text { PL13W } & 0.10924409 \mathrm{~W} \\ \text { SFO2 } & 400.1320007\end{array}$

F2 - Processing parameters

S1 - Processing parameters

$\begin{array}{lr}\text { WF } \\ \text { WSB } & 100.6127572 \mathrm{MH} \\ \text { SSB } & 0 \quad \text { EM }\end{array}$

$\underset{G B}{\mathrm{LB}} \quad 0 \mathrm{~Hz}$

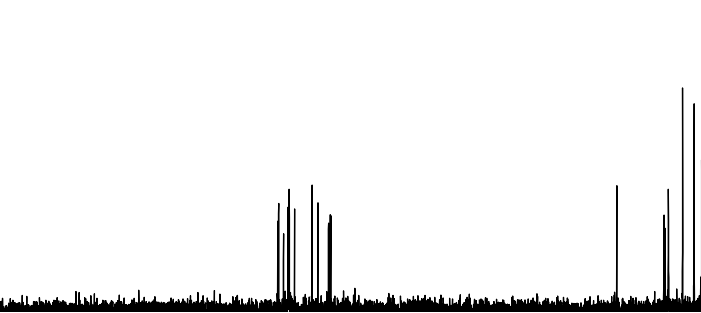

1.00

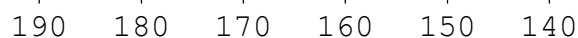<smiles>CC[C@H](COC(=O)OC)OC</smiles>

$\mathrm{AcO}$

$\begin{array}{lll}\mathrm{BzO} & 24 & \mathrm{O} \\ \mathrm{H} & \mathrm{OAc} \quad \mathrm{AcO}\end{array}$ 
${ }^{1} \mathrm{H}$ NMR $\left(400 \mathrm{MHz}, \mathrm{CDCl}_{3}\right)$ of $\mathbf{2 5}$ :

Я

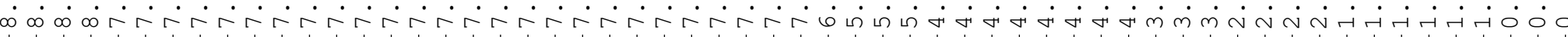

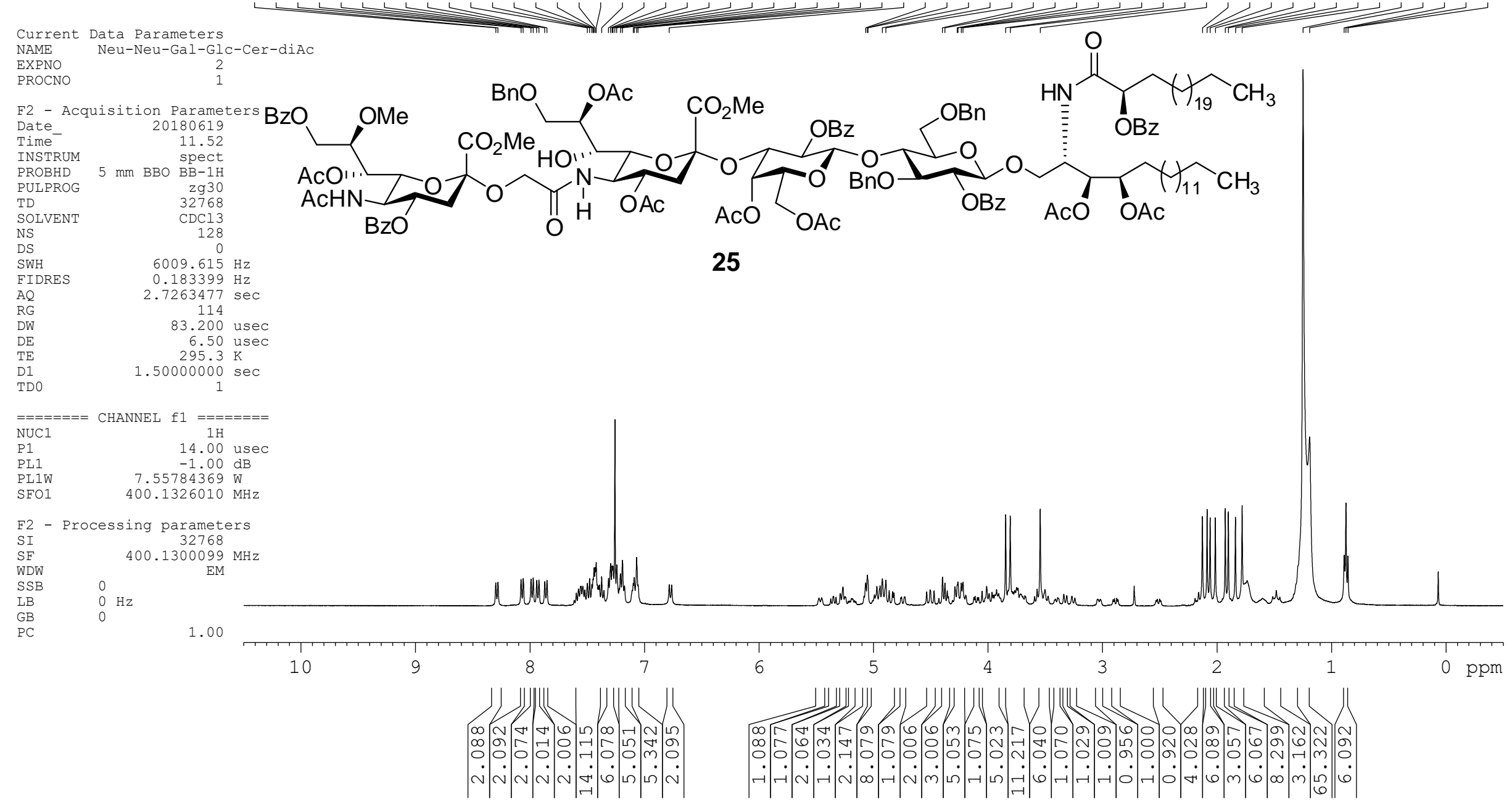


${ }^{1} \mathrm{H}$ NMR (400 MHz, $\mathrm{CDCl}_{3}$ ) of $\mathbf{2 5}$ :

Current Data Parameters

NAME Neu-Neu-Gal-Glc-Cer-diAc

EXPNO
PROCNO

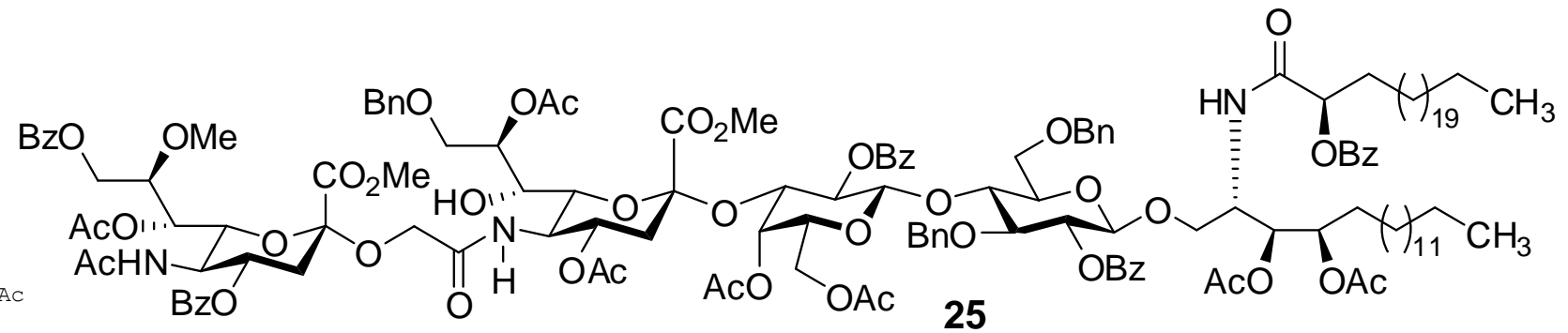

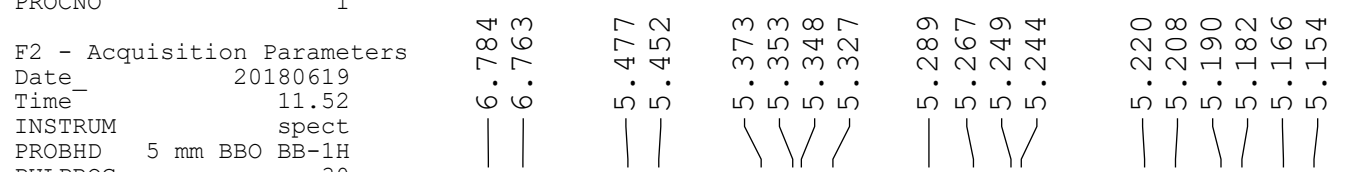

25

PULPROG

TD
SOLVENT
NS

NS
DS

SWH
FIDRES

FIDR
$A Q$
RG

AQ
RG
DW

$\mathrm{RG}$
$\mathrm{DW}$
$\mathrm{DE}$
$\mathrm{TE}$
$\mathrm{D} 1$

D1 $\quad 1.50000000 \mathrm{Kec}$

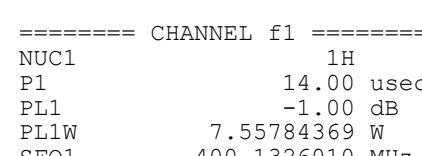

SFO1 $400.1326010 \mathrm{MHz}$

F2 - Processing parameters

$\begin{array}{lr}\text { SI } & 32768 \\ \text { SF } & 400.1300099 \mathrm{MHz}\end{array}$

$\begin{array}{lr}\text { SF } & 400.1300099 \mathrm{MHz} \\ \text { WDW } & \text { EM }\end{array}$

$\begin{array}{ll}\text { SSB } & 0 \\ \text { LB } & 0 \\ \text { GB } & 0\end{array}$

1.00
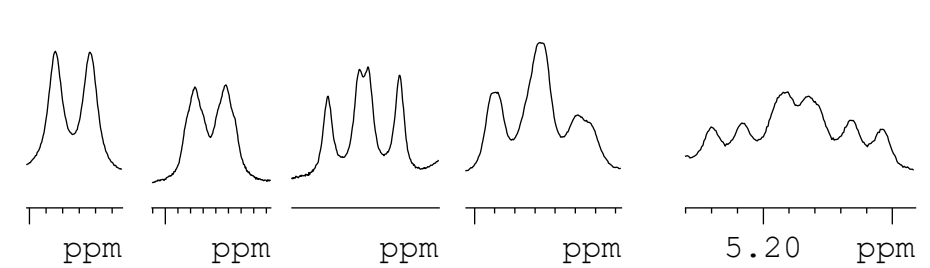

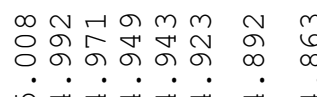

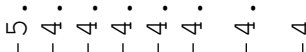

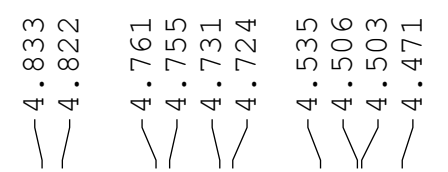

๙ูำ

मं वंन में
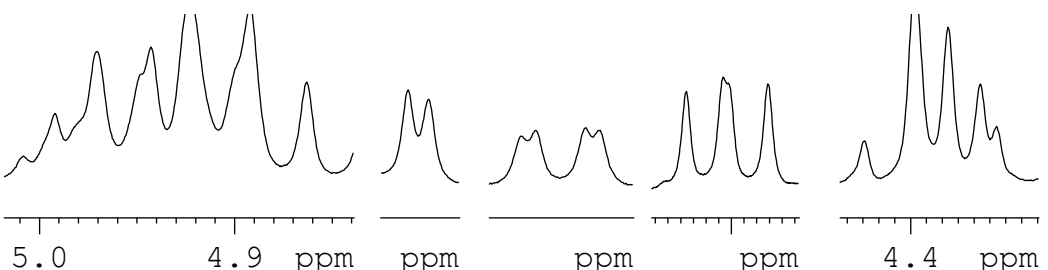

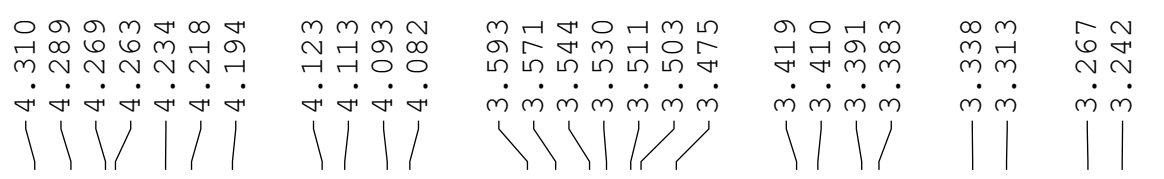

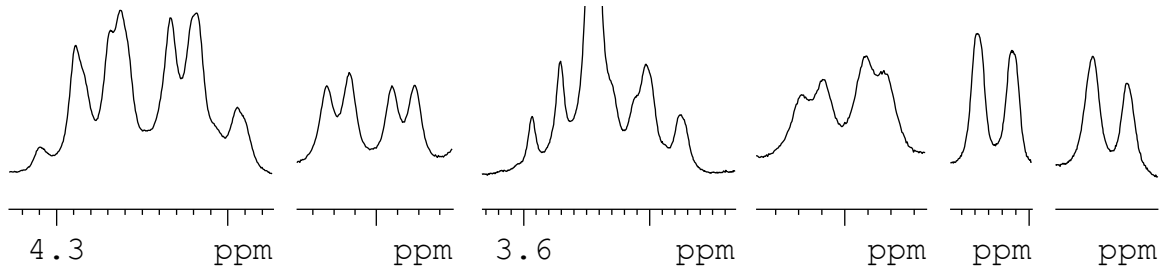

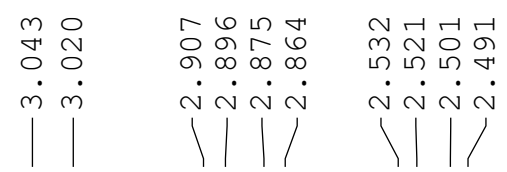

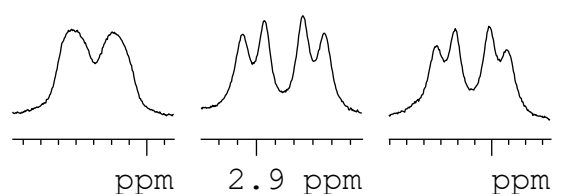


${ }^{13} \mathrm{C}$, DEPT-90 and DEPT-135 NMR (101 MHz, $\left.\mathrm{CDCl}_{3}\right)$ of 25:

Current Data Parameters
NAME Neu-Neu-Gal-Glc-
Cer-diAc

$\begin{array}{ll}\text { Cer-diAc } & 8 \\ \text { EXNO } & 1\end{array}$

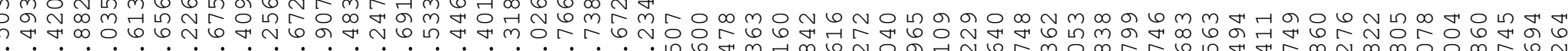

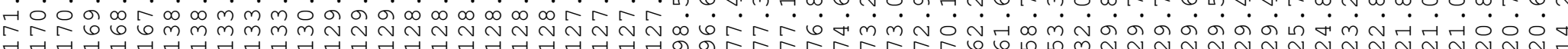

F2 - Acquisition Parameters

Date_ 2018062

Time ${ }^{-}$

9.07
spect
B -14

PROBHD

PROBHD
PULPROG

SOLVENT

NS
DS

SWH
FIDRES

AQ
RG
DW

DE

TE
D1
D11

TD0

NUC1

$5 \mathrm{~mm} \mathrm{BBO} \mathrm{BB}-1 \mathrm{H}$

zgpg30
65536
CDC13
25551

$20161.291 \mathrm{~Hz}$ $1.6253428 \mathrm{sec}$

2050
24.800 usec

24.800 usec
6.50 usec

$296.3 \mathrm{~K}$

$1.50000000 \mathrm{sec}$
$0.03000000 \mathrm{sec}$

CHANNEL $\mathrm{f}$

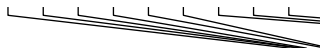

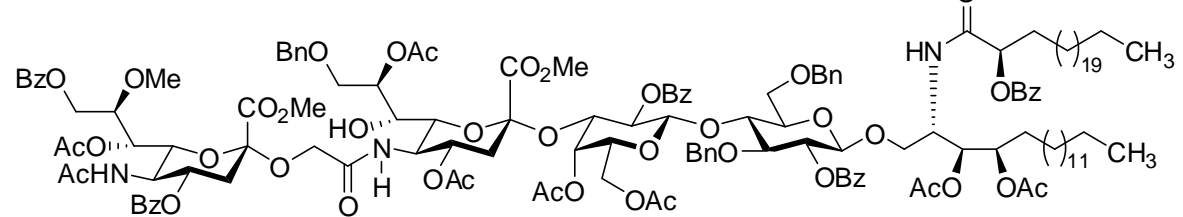

$\begin{array}{ll}\text { PLIW } & 31.64976883 \mathrm{~W} \\ \text { SFO1 } & 100.6228303 \mathrm{MHz}\end{array}$

$=======$ CHANNEL f2 $=======$

CPDPRG2 waltz16

$\begin{array}{ll}\text { NUC2 } & 1 \mathrm{H} \\ \text { PCPD2 } & 90.00 \mathrm{usec} \\ \text { PL2 } & -1.00 \mathrm{~dB}\end{array}$

$\begin{array}{ll}\text { PL2 } & -1.00 \mathrm{~dB} \\ \text { PL12 } & 15.16 \mathrm{~dB} \\ \text { PL13 } & 17.40 \mathrm{~dB}\end{array}$

$\begin{array}{ll}\text { PL13 } & 15.45 \mathrm{~dB} \\ \text { PL2W } & 7.55784369 \mathrm{~W} \\ \text { PL12W } & 0.18297760 \mathrm{~W}\end{array}$

PL13W

$4.10924409 \mathrm{~W}$

F2 - Processing parameters

$\begin{array}{lc}\text { SI } & 32768 \\ \text { SF } & 100.6127574 \mathrm{MHz} \\ \text { WDW } & \text { EM }\end{array}$

$\begin{array}{ll}\text { SSB } & 0 \\ \text { LB } & 0\end{array}$

GB

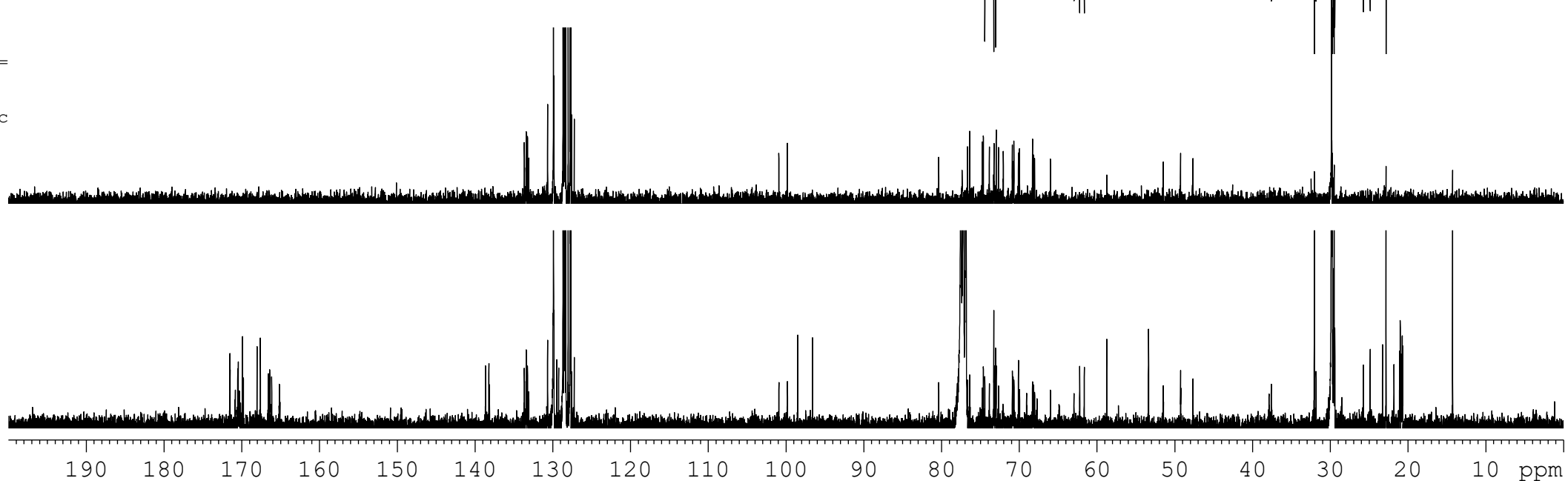




\section{${ }^{1} \mathrm{H}$ NMR $\left(700 \mathrm{MHz}, \mathrm{CD}_{3} \mathrm{OD}\right)$ of LLG-3:}

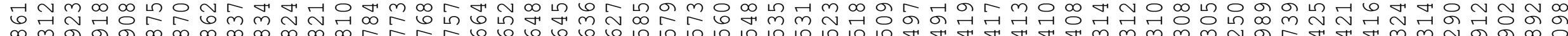

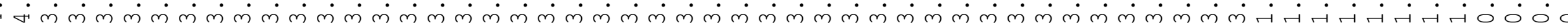

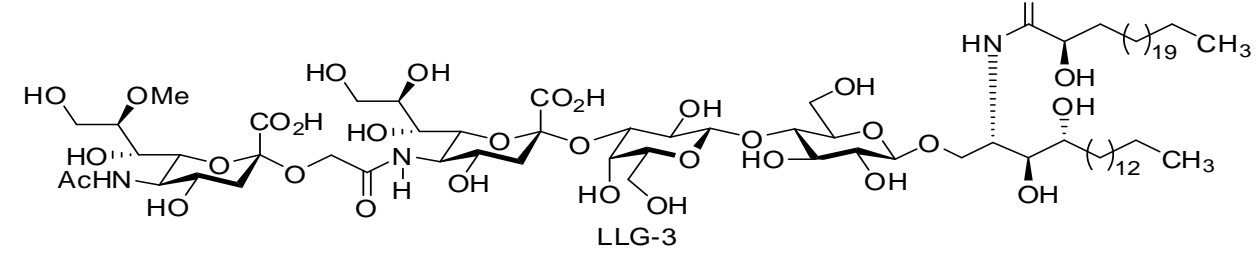

Current Data Parameters

PROCNO

F2 - Processing parameters

SF $699.7455748 \mathrm{MHz}$

$\begin{array}{lll}\mathrm{SSB} & 0 & 0.30 \mathrm{~Hz} \\ \mathrm{LB} & 0\end{array}$

PC
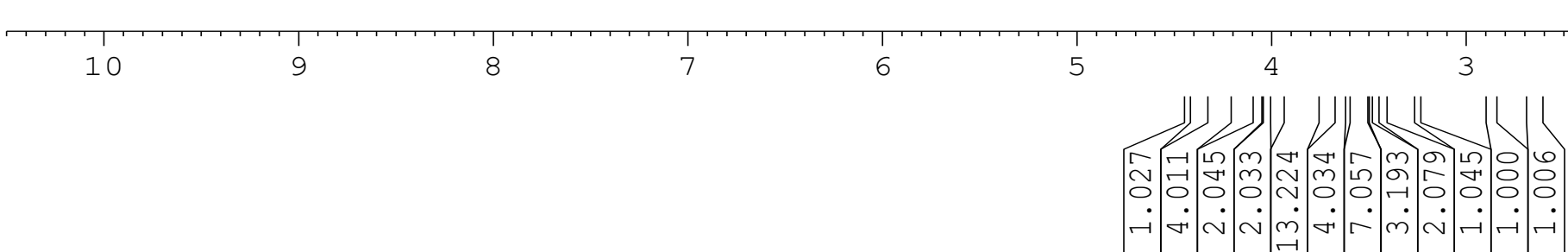

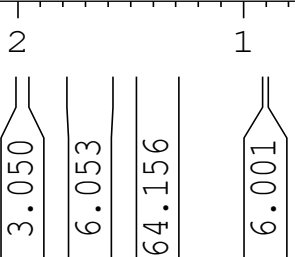

0 ppm 
${ }^{13} \mathrm{C}$, DEPT-90 and DEPT-135 NMR (176 MHz, $\left.\mathrm{CD}_{3} \mathrm{OD}\right)$ of LLG-3:

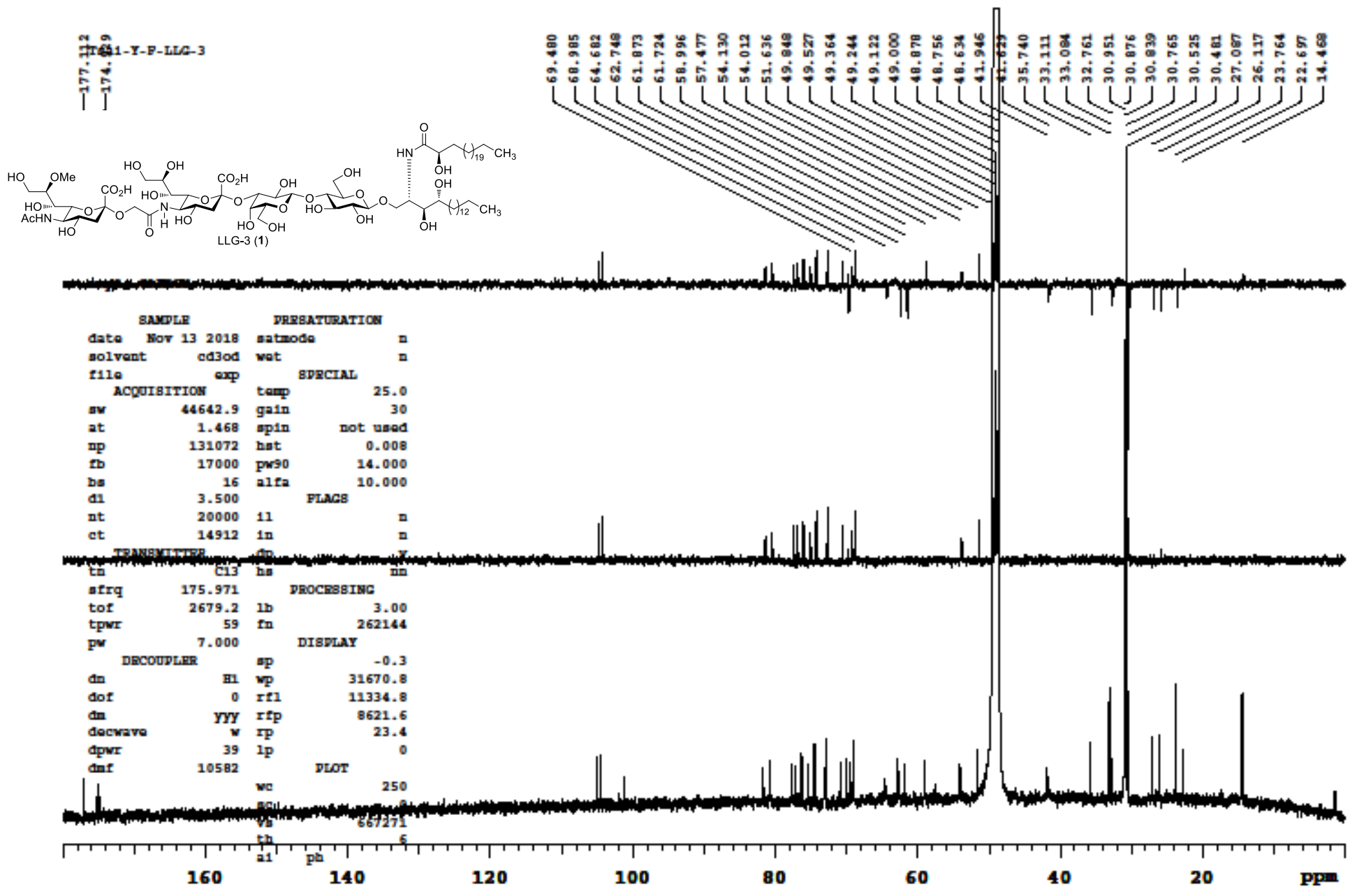


${ }^{13} \mathrm{C}$, DEPT-90 and DEPT-135 NMR (176 MHz, CD 3 OD) of LLG-3:

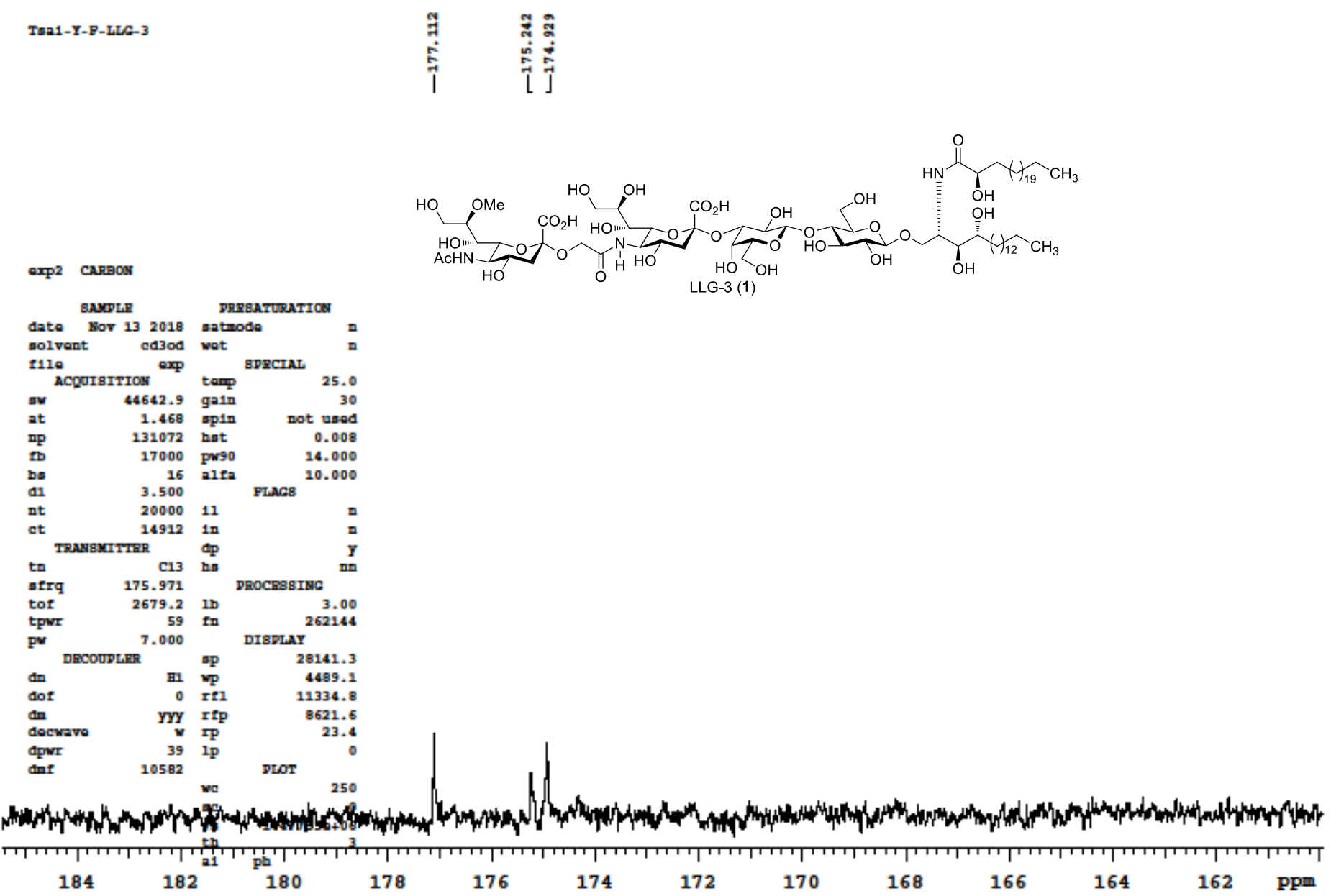


${ }^{13} \mathrm{C}$, DEPT-90 and DEPT-135 NMR (176 MHz, $\left.\mathrm{CD}_{3} \mathrm{OD}\right)$ of LLG-3:
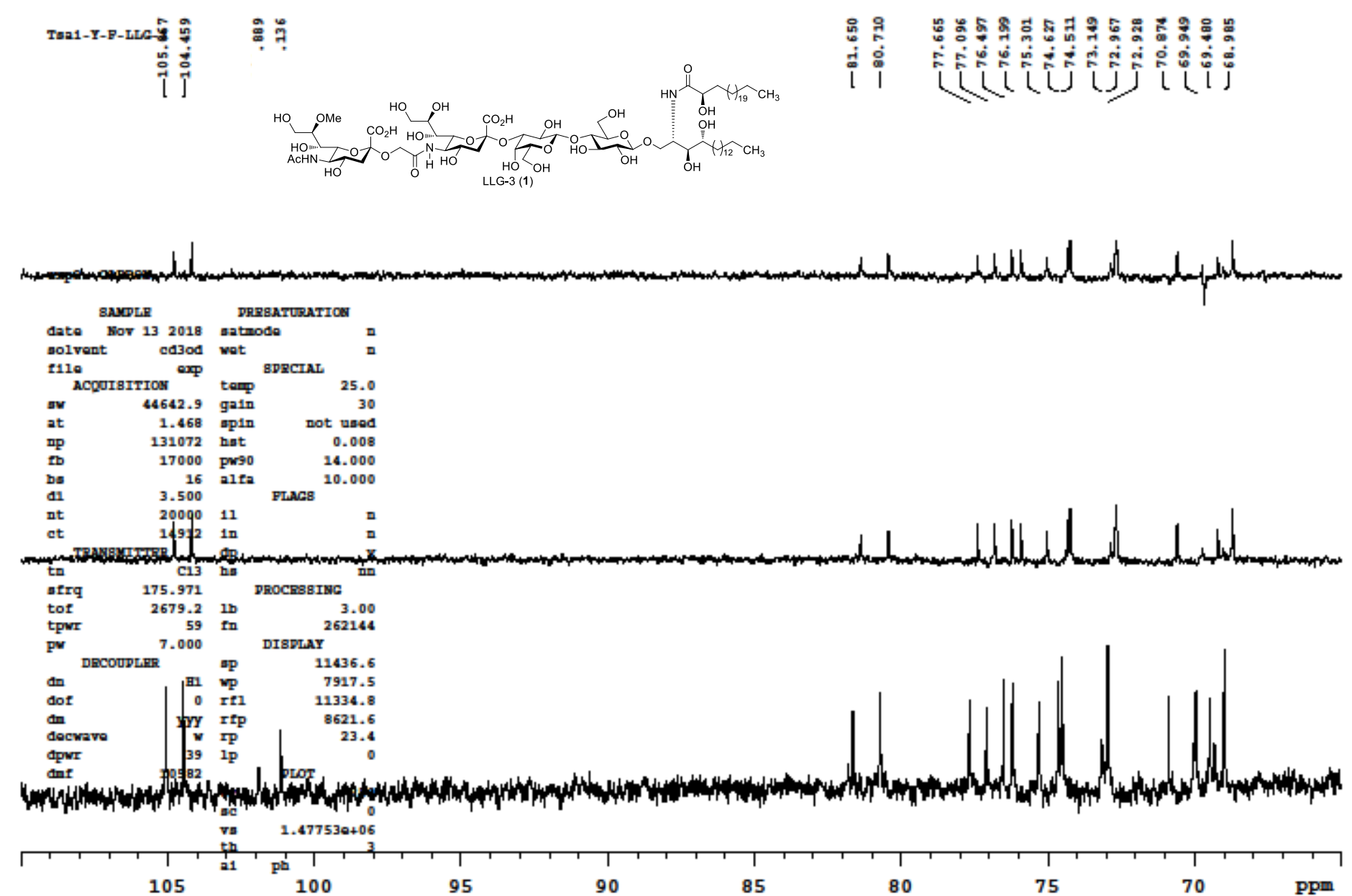